\title{
Enantioselective Copper-Catalyzed Desymmetrization of 1,3-Diketones Involving Borylation of Styrenes
}

Purui Zheng, Xiaoyu Han, Jiao Hu, Xiaoming Zhao, ${ }^{*}$ and Tao XU *

Shanghai Key Laboratory of Chemical Assessment and Sustainability, School of Chemical Science and Engineering, Tongji University, 1239 Siping Road, Shanghai, 200092, P. R. of China.

E-mail: taoxu@tongji.edu.cn;

xmzhao08@mail.tongji.edu.cn.

Content

1. General considerations................................................ 2

2. General procedure for preparation of starting material.................. S2

3. Screening results.................................................... 7

4. General procedure for catalytic asymmetric cyclization of 1,3-

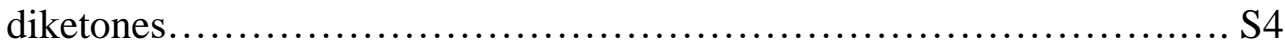

5. The functional-group compatibility study......................... 9

6. General procedures for the transformations of the enantioenriched cyclic boronic ester products........................................... S11

7. The characterization of the new products.............................. 15

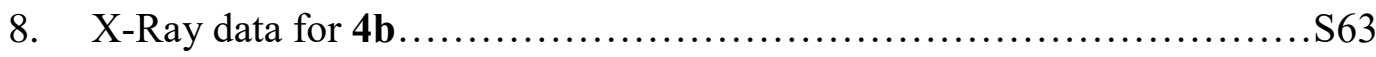

9. Spectroscopic data............................................. S66 


\section{Supporting Information}

\section{General Considerations.}

All commercially available compounds were used as received. ${ }^{1} \mathrm{H}$ and ${ }^{13} \mathrm{C}$ spectra were recorded on a Bruker Avance 400, 600 spectrometer, and $\mathrm{CDCl}_{3}$ was purchased from Aldrich. The chemical shifts $(\delta)$ are given in parts per million relative to internal standard TMS (0 ppm for $\left.{ }^{1} \mathrm{H}\right), \mathrm{CDCl}_{3}\left(77.0 \mathrm{ppm}\right.$ for $\left.{ }^{13} \mathrm{C}\right)$. Flash column chromatography was performed on silica gel 60 (particle size 200-400 mesh ASTM, purchased from Yantai, China) and eluted with petroleum ether/ethyl acetate. Solvents were dried and purified according to the procedure from 'Purification of Laboratory Chemicals book'. Chiral HPLC analyses were performed on UltiMate 3000 liquid chromatography. Unless otherwise noted, all other reagents and starting materials were purchased from commercial sources and used without further purification.

\section{General Procedure for Preparation of Starting Material}

\subsection{General Procedure for synthesis of 1a-1h}

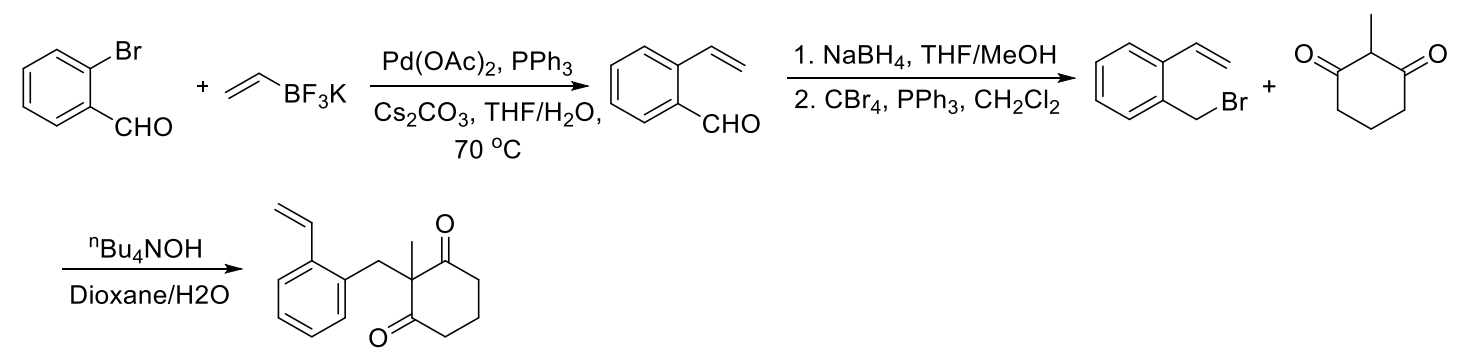

According to the literature ${ }^{\mathrm{S} 1}$, an oven-dried $100 \mathrm{~mL}$ round bottom flask with magnetic stir bar was charged with $\mathrm{Pd}(\mathrm{OAc})_{2}(0.1 \mathrm{mmol}, 1 \mathrm{~mol} \%), \mathrm{PPh}_{3}(0.2 \mathrm{mmol}, 2$ mol \%), $\mathrm{Cs}_{2} \mathrm{CO}_{3}$ (30 mmol, 3 equiv.) and THF ( $50 \mathrm{~mL}$ ) under nitrogen atmosphere. The mixture was stirred for $20 \mathrm{~min}$. Then aldehyde $(10 \mathrm{mmol})$, vinyl trifluoroborate potassium (12 mmol, 1.2 equiv.) and degassed water $(5 \mathrm{~mL})$ were added and the reaction was stirred for another $10 \mathrm{~min}$ at room temperature. The reaction mixture was stirred at $70{ }^{\circ} \mathrm{C}$ overnight. Upon completion, the reaction was cooled to room temperature, quenched with water and diluted with ethyl acetate. The layers were allowed to separated and the aqueous layer was extracted with ethyl acetate three times. The organic layers were combined, dried over anhydrous $\mathrm{Na}_{2} \mathrm{SO}_{4}$ and concentrated under reduced pressure. The residue was purified by flash column chromatography on silica gel with a gradient eluent of petroleum ether/ethyl acetate affording the 2vinylbenzaldehyde.

To a stirred solution of 2-vinylbenzaldehyde $(10 \mathrm{mmol})$ in THF/MeOH $(24 \mathrm{~mL}$, $\mathrm{v} / \mathrm{v}=5 / 1)$ was added slowly $\mathrm{NaBH}_{4}(20 \mathrm{mmol}, 2$ equiv. $)$ at $0{ }^{\circ} \mathrm{C}$. And the mixture was

S1 Jarrige L.; Carboni A., Dagousset G.; Levitre G., Magnier E.; Masson G. Org. Lett. 2016, 18, 2906. 
stirred until completion (monitored by TLC analysis). The solution was diluted with ethyl acetate and quenched with $10 \%$ aqueous $\mathrm{HCl}$ carefully. The layers were allowed to separated and the aqueous layer was extracted with ethyl acetate three times. The organic layers were combined, dried over anhydrous $\mathrm{Na}_{2} \mathrm{SO}_{4}$ and concentrated under reduced pressure. The residue was used without further purified.

To a stirred solution of $\mathrm{CBr}_{4}(15 \mathrm{mmol}, 1.5$ equiv. $)$ in DCM $(20 \mathrm{~mL})$ was added $\mathrm{PPh}_{3}\left(15 \mathrm{mmol}, 1.5\right.$ equiv.) in portions at $0{ }^{\circ} \mathrm{C}$. The suspension was allowed to worm to room temperature and the alcohol $(10 \mathrm{mmol})$ was added. The mixture was stirred until completion (monitored by TLC analysis). The reaction solution was concentrated under reduced pressure. The residue was purified by flash column chromatography on silica gel with a gradient eluent of petroleum ether/ethyl acetate to affording 1(bromomethyl)-2-vinylbenzene.

To a stirred solution of 2-methylcyclohexane-1,3-dione (6 mmol, 1.2 equiv.) in dioxane $(5 \mathrm{~mL})$ was added aqueous ${ }^{n} \mathrm{Bu}_{4} \mathrm{NOH}\left(40 \%\right.$ in $\left.\mathrm{H}_{2} \mathrm{O}, 3.3 \mathrm{~mL}\right)$. The mixture was stirred for $20 \mathrm{~min}$ at room temperature. Then a solution of 1-(bromomethyl)-2vinylbenzene $(5 \mathrm{mmol})$ in dioxane $(5 \mathrm{~mL})$ was added and the reaction mixture was stirred for another $6 \mathrm{~h}$. Upon completion, the reaction was quenched with aqueous $\mathrm{NH}_{4} \mathrm{Cl}$ and extracted with ethyl acetate three times. The organics were combined, dried over anhydrous $\mathrm{Na}_{2} \mathrm{SO}_{4}$, concentrated under reduced pressure. The residue was purified by flash column chromatography on silica gel with a gradient eluent of petroleum ether/ethyl acetate to affording the final product.

\subsection{General Procedure for synthesis of $1 \mathrm{i}$}

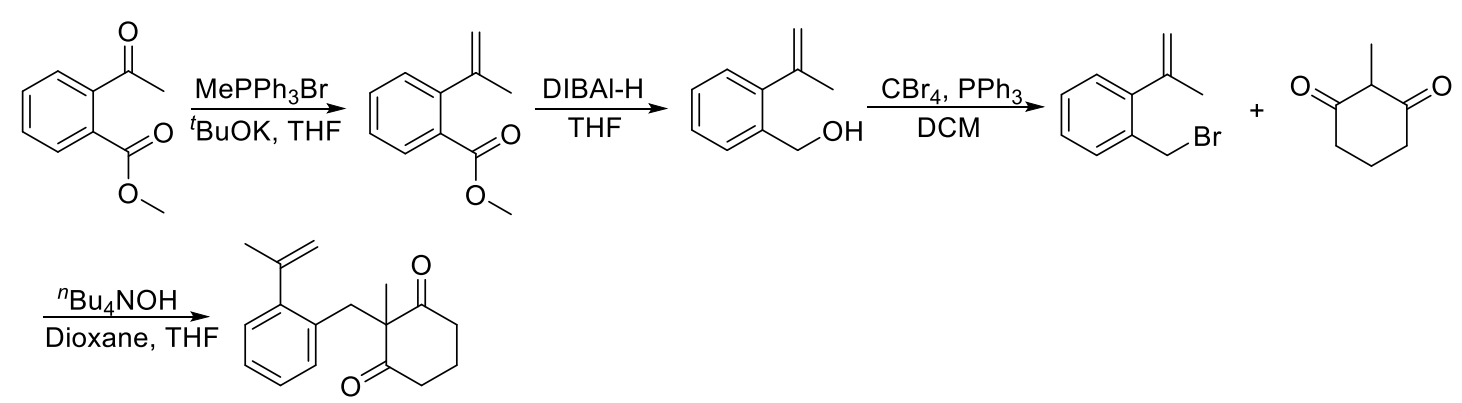

An oven-dried $250 \mathrm{~mL}$ round bottom flask equipped with magnetic stir bar was charged with methyltriphenylphosphonium bromide (30 mmol, 1.5 equiv.) and THF $(100 \mathrm{~mL})$ under nitrogen atmosphere at $0{ }^{\circ} \mathrm{C} .{ }^{t} \mathrm{BuOK}$ (30 mmol, 1.5 equiv.) was added slowly and the suspension was stirred for $30 \mathrm{~min}$. Then a solution of methyl 2acetylbenzoate $(20 \mathrm{mmol})$ in THF $(10 \mathrm{~mL})$ was added to the reaction mixture. Upon completion, the reaction was quenched with aqueous $\mathrm{NH}_{4} \mathrm{Cl}$ and extracted with ethyl acetate three times. The organics were combined, dried over anhydrous $\mathrm{Na}_{2} \mathrm{SO}_{4}$, concentrated under reduced pressure. The residue was purified by flash column chromatography on silica gel with a gradient eluent of petroleum ether/ethyl acetate to affording the corresponding styrene in $60 \%$ yield.

To a stirred solution of methyl 2-(prop-1-en-2-yl) benzoate $(10 \mathrm{mmol})$ in anhydrous THF (30 mL) was added slowly DIBAl-H (25 mmol, 2.5 equiv., $1 \mathrm{M}$ in hexanes) under nitrogen atmosphere at $-60{ }^{\circ} \mathrm{C}$. Then the solution was allowed to worm to room 
temperature and stirred for $2 \mathrm{~h}$. The reaction was diluted with ethyl acetate and quenched with $10 \%$ aqueous $\mathrm{HCl}$ carefully at $0{ }^{\circ} \mathrm{C}$. The layers were allowed to separated and the aqueous layer was extracted with ethyl acetate three times. The organic layers were combined, dried over anhydrous $\mathrm{Na}_{2} \mathrm{SO}_{4}$ and concentrated under reduced pressure. The residue was used without purified.

To a stirred solution of $\mathrm{CBr}_{4}(15 \mathrm{mmol}, 1.5$ equiv. $)$ in DCM $(20 \mathrm{~mL})$ was added $\mathrm{PPh}_{3}$ (15 mmol, 1.5 equiv.) in portions at $0{ }^{\circ} \mathrm{C}$. The suspension was allowed to worm to room temperature and the alcohol $(10 \mathrm{mmol})$ was added. The mixture was stirred until completion (monitored by TLC analysis). The reaction solution was concentrated under reduced pressure. The residue was purified by flash column chromatography on silica gel with a gradient eluent of petroleum ether/ethyl acetate to affording 1(bromomethyl)-2-(prop-1-en-2-yl) benzene in 80\% yield with two steps.

To a stirred solution of 2-methylcyclohexane-1,3-dione (6 mmol, 1.2 equiv.) in dioxane $(5 \mathrm{~mL})$ was added aqueous ${ }^{n} \mathrm{Bu} 4 \mathrm{NOH}\left(40 \%\right.$ in $\left.\mathrm{H}_{2} \mathrm{O}, 3.3 \mathrm{~mL}\right)$. The mixture was stirred for $20 \mathrm{~min}$ at room temperature. Then a solution of 1-(bromomethyl)-2-(prop-1en-2-yl) benzene $(5 \mathrm{mmol})$ in dioxane $(5 \mathrm{~mL})$ was added and the reaction mixture was stirred for another $6 \mathrm{~h}$. Upon completion, the reaction was quenched with aqueous $\mathrm{NH}_{4} \mathrm{Cl}$ and extracted with ethyl acetate three times. The organics were combined, dried over anhydrous $\mathrm{Na}_{2} \mathrm{SO}_{4}$, concentrated under reduced pressure. The residue was purified by flash column chromatography on silica gel with a gradient eluent of petroleum ether/ethyl acetate to affording the final product ( $243 \mathrm{mg}, 19 \%$ yield).

\subsection{General Procedure for synthesis of 3a-3h}

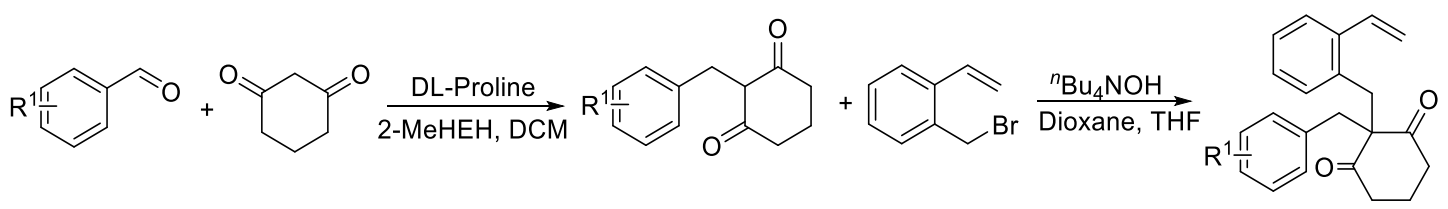

According to the literature ${ }^{\mathrm{S} 2}$, an oven-dried $250 \mathrm{~mL}$ round bottom flask equipped with magnetic stir bar was charged with 1,3-cyclohexanedione (30 mmol), Hantzsh ester (33 mmol, 1.1 equiv.), DL-Proline (6 mmol, $20 \mathrm{~mol} \%$ ) and DCM (50 mL). A solution of aldehyde (33 mmol, 1.1 equiv.) in DCM $(5 \mathrm{~mL})$ was added and the reaction mixture was refluxed at $70{ }^{\circ} \mathrm{C}$ overnight. Upon completion, the reaction was allowed to cool to room temperature and the solvent was removed. The residue was purified by flash column chromatography on silica gel with a gradient eluent of petroleum ether/ethyl acetate to affording the 2-substituent-cyclohexane-1,3-dione.

To a stirred solution of 2-substituent-cyclohexane-1,3-dione (6 mmol, 1.2 equiv.) in dioxane $(5 \mathrm{~mL})$ was added aqueous ${ }^{n} \mathrm{Bu}_{4} \mathrm{NOH}\left(40 \%\right.$ in $\left.\mathrm{H}_{2} \mathrm{O}, 3.3 \mathrm{~mL}\right)$. The mixture was stirred for $20 \mathrm{~min}$ at room temperature. Then a solution of 1-(bromomethyl)-2(prop-1-en-2-yl) benzene $(5 \mathrm{mmol})$ in dioxane $(5 \mathrm{~mL})$ was added and the reaction mixture was stirred for another $6 \mathrm{~h}$. Upon completion, the reaction was quenched with aqueous $\mathrm{NH}_{4} \mathrm{Cl}$ and extracted with ethyl acetate three times. The organics were combined, dried over anhydrous $\mathrm{Na}_{2} \mathrm{SO}_{4}$, concentrated under reduced pressure. The residue was purified by flash column chromatography on silica gel with a gradient eluent of petroleum ether/ethyl acetate to affording the final product.

S2 Zhu C.; Wang D.; Zhao Y.; Sun W. Y.; Shi Z. J. Am. Chem. Soc. 2017, 139, 16486. 
2.4 General Procedure for synthesis of $3 i^{\mathrm{S} 3}$

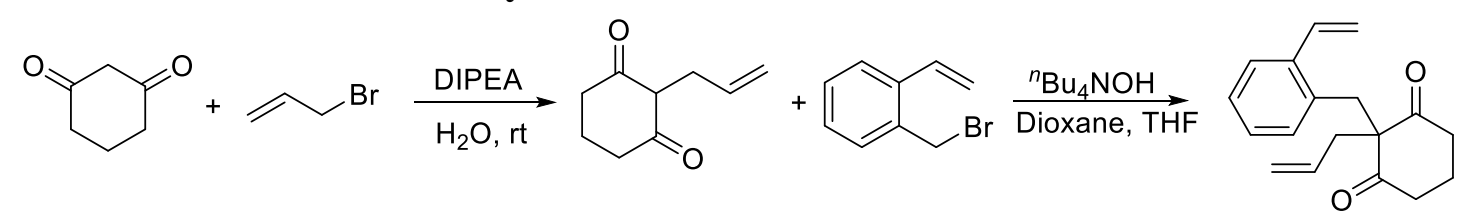

Cyclohexane-1,3-dione (30 mmol, 1.5 equiv.) and DIPEA (24 mmol, 1.2 equiv.) were dissolved in water $(20 \mathrm{~mL})$. The solution was stirred for $20 \mathrm{~min}$ followed by addition of allyl bromide $(20 \mathrm{mmol})$. Upon completion, the reaction mixture was acidified by $10 \%$ aqueous $\mathrm{HCl}$. The suspension was extracted with ethyl acetate three times. The organics was combined, dried over anhydrous $\mathrm{Na}_{2} \mathrm{SO}_{4}$ and concentrated under reduced pressure. The residue was purified by flash column chromatography on silica gel with a gradient eluent of petroleum ether/ethyl acetate to affording 2allylcyclohexane-1,3-dione in 55\% yield.

To a stirred solution of 2-allylcyclohexane-1,3-dione (6 mmol, 1.2 equiv.) in dioxane $(5 \mathrm{~mL})$ was added aqueous ${ }^{n} \mathrm{Bu}_{4} \mathrm{NOH}\left(40 \%\right.$ in $\left.\mathrm{H}_{2} \mathrm{O}, 3.3 \mathrm{~mL}\right)$. The mixture was stirred for $20 \mathrm{~min}$ at room temperature. Then a solution of 1-(bromomethyl)-2-(prop-1en-2-yl) benzene $(5 \mathrm{mmol})$ in dioxane $(5 \mathrm{~mL})$ was added and the reaction mixture was stirred for another $6 \mathrm{~h}$. Upon completion, the reaction was quenched with aqueous $\mathrm{NH}_{4} \mathrm{Cl}$ and extracted with ethyl acetate three times. The organics were combined, dried over anhydrous $\mathrm{Na}_{2} \mathrm{SO}_{4}$, concentrated under reduced pressure. The residue was purified by flash column chromatography on silica gel with a gradient eluent of petroleum ether/ethyl acetate to affording the final product ( $335 \mathrm{mg}, 25 \%$ yield).

\subsection{General Procedure for synthesis of $3 \mathbf{j}^{\mathrm{S} 4}$}

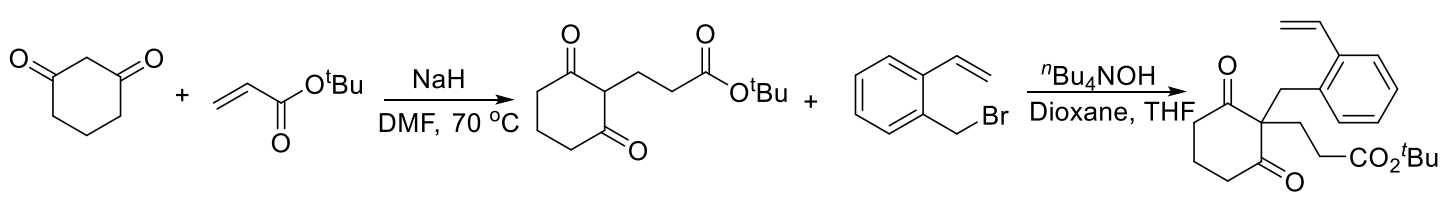

An oven-dried $100 \mathrm{~mL}$ round bottom flask equipped with magnetic stir bar was added with $60 \% \mathrm{NaH}$ (24 mmol, 1.2 equiv.) and DMF (40 mL) under nitrogen atmosphere at $0{ }^{\circ} \mathrm{C}$. A solution of cyclohexane-1,3-dione $(20 \mathrm{mmol})$ in DMF $(10 \mathrm{~mL})$ was added slowly and the reaction was stirred for $30 \mathrm{~min}$. Then $t$-butyl acrylate (30 mmol, 1.5 equiv.) was added and the reaction was heated at $70{ }^{\circ} \mathrm{C}$ for $4 \mathrm{~h}$. Upon completion, the reaction mixture was allowed to cool to room temperature and acidified by $10 \%$ aqueous $\mathrm{HCl}$. The suspension was extracted with ethyl acetate three times. The organics was combined, dried over anhydrous $\mathrm{Na}_{2} \mathrm{SO}_{4}$ and concentrated under reduced pressure. The residue was purified by flash column chromatography on silica gel with a gradient eluent of petroleum ether/ethyl acetate to affording $t$-butyl 3-(2,6dioxocyclohexyl) propanoate in $73 \%$ yield.

To a stirred solution of $t$-butyl 3-(2,6-dioxocyclohexyl) propanoate $(6 \mathrm{mmol}, 1.2$ equiv.) in dioxane $(5 \mathrm{~mL})$ was added aqueous ${ }^{n} \mathrm{Bu} 4 \mathrm{NOH}\left(40 \%\right.$ in $\left.\mathrm{H}_{2} \mathrm{O}, 3.3 \mathrm{~mL}\right)$. The mixture was stirred for $20 \mathrm{~min}$ at room temperature. Then a solution of 1-

\footnotetext{
S3 Wu X.; Chen Z.; Bai Y. B.; Dong V. M. J. Am. Chem. Soc. 2016, 138, 12013.

S4 Konno M.; Nakae T.; Hamanaaka N. SynLett. 1997,1472.
} 
(bromomethyl)-2-(prop-1-en-2-yl) benzene $(5 \mathrm{mmol})$ in dioxane $(5 \mathrm{~mL})$ was added and the reaction mixture was stirred for another $6 \mathrm{~h}$. Upon completion, the reaction was quenched with aqueous $\mathrm{NH}_{4} \mathrm{Cl}$ and extracted with ethyl acetate three times. The organics were combined, dried over anhydrous $\mathrm{Na}_{2} \mathrm{SO}_{4}$, concentrated under reduced pressure. The residue was purified by flash column chromatography on silica gel with a gradient eluent of petroleum ether/ethyl acetate to affording the final product ( $409 \mathrm{mg}$, $23 \%$ yield).

\subsection{General Procedure for synthesis of $3 \mathbf{k}^{\mathrm{S} 5}$}

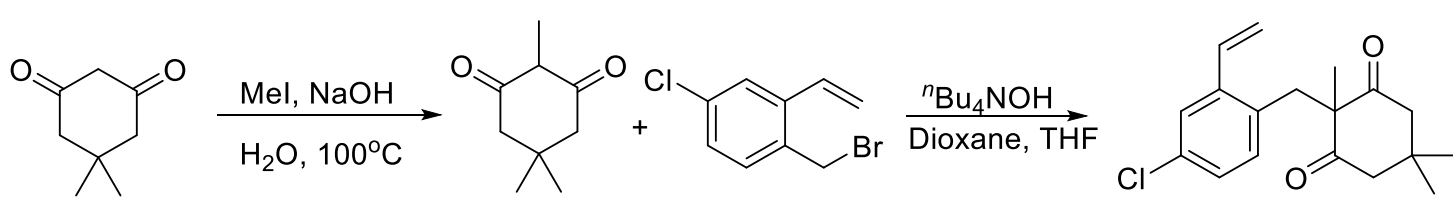

$\mathrm{NaOH}$ (24 mmol, 1.2 equiv.) was dissolved in water $(8 \mathrm{~mL})$. 5,5dimethylcyclohexane-1,3-dione $(20 \mathrm{mmol})$ was added at $0{ }^{\circ} \mathrm{C}$ and the solution was stirred for $30 \mathrm{~min}$. MeI (40 mmol, 2 equiv.) was added and the reaction mixture was heated at $100{ }^{\circ} \mathrm{C}$ for $14 \mathrm{~h}$. Then the reaction was allowed to cool to $0{ }^{\circ} \mathrm{C}$. The precipitate was collected, washed with cold water and petroleum ether and dried in vacuum. The pale-yellow solid was used without further purification.

To a stirred solution of 2-methyl-5,5-dimethylcyclohexane-1,3-dione (6 mmol, 1.2 equiv.) in dioxane $(5 \mathrm{~mL})$ was added aqueous ${ }^{n} \mathrm{Bu} 4 \mathrm{NOH}\left(40 \%\right.$ in $\left.\mathrm{H}_{2} \mathrm{O}, 3.3 \mathrm{~mL}\right)$. The mixture was stirred for $20 \mathrm{~min}$ at room temperature. Then a solution of 1(bromomethyl)-2-(prop-1-en-2-yl)-4-chlorobenzene $(5 \mathrm{mmol})$ in dioxane $(5 \mathrm{~mL})$ was added and the reaction mixture was stirred for another $6 \mathrm{~h}$. Upon completion, the reaction was quenched with aqueous $\mathrm{NH}_{4} \mathrm{Cl}$ and extracted with ethyl acetate three times. The organics were combined, dried over anhydrous $\mathrm{Na}_{2} \mathrm{SO}_{4}$, concentrated under reduced pressure. The residue was purified by flash column chromatography on silica gel with a gradient eluent of petroleum ether/ethyl acetate to affording the final product (304 mg, 20\% yield).

\subsection{General Procedure for synthesis of $5^{\text {S6 }}$}

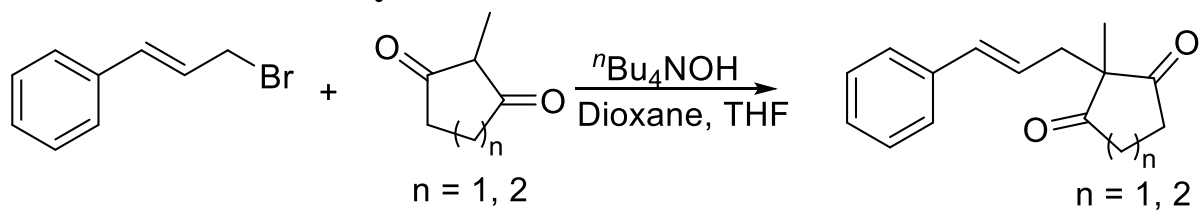

To a stirred solution of 2-methylcyclohexane-1,3-dione (6 mmol, 1.2 equiv.) in dioxane $(5 \mathrm{~mL})$ aqueous ${ }^{n} \mathrm{Bu} 4 \mathrm{NOH}\left(40 \%\right.$ in $\left.\mathrm{H}_{2} \mathrm{O}, 3.3 \mathrm{~mL}\right)$ was added. The mixture was stirred for $20 \mathrm{~min}$ at room temperature. Then a solution of (E)-(3-bromoprop-1-en-1-yl) benzene $(5 \mathrm{mmol})$ in dioxane $(5 \mathrm{~mL})$ was added and the reaction mixture was stirred for another $6 \mathrm{~h}$. Upon completion, the reaction was quenched with aqueous $\mathrm{NH}_{4} \mathrm{Cl}$ and extracted with ethyl acetate three times. The organics were combined, dried over anhydrous $\mathrm{Na}_{2} \mathrm{SO}_{4}$, concentrated under reduced pressure. The residue was purified by

\footnotetext{
S5 Moser R.; Boskovic Z. V.; Crowe C. S.; Lipshutz B. H. J. Am. Chem. Soc. 2010, 132, 7852.

s6 Byrne S. J.; Fletcher A. J.; Hebeisen P.; Willis M. C. Org. Biomol. Chem. 2010, 8, 758.
} 
flash column chromatography on silica gel with a gradient eluent of petroleum ether/ethyl acetate to affording the corresponding product.

\section{Screening Results.}<smiles>C=Cc1ccccc1CC1(C)C(=O)CCCC1=O</smiles>

1a

$$
\begin{gathered}
\text { Cu cat.(x mol\%) } \\
\text { Ligand }(1.2 \times \text { mol } \%) \\
\underset{{ }^{t} \text { BuOLi (2 equiv.) }}{\mathrm{B}_{2} \operatorname{Pin}_{2} \text { (2 equiv.) }}
\end{gathered}
$$
solvent, $15-20^{\circ} \mathrm{C}$<smiles>O=C1CCC[C@@]2(O)[C@@H]1Cc1ccccc1[C@H]2Cc1ccccc1</smiles>

2a<smiles>CC12Cc3ccccc3C3CB(O)OC31CCCC2=O</smiles>

$2 a^{\prime}$

The Standard condition was listed below and the screening experiments were tested by changing the relevant parameters based on this procedure:

In the nitrogen filled glovebox, A $10 \mathrm{~mL}$ oven-dried Schlenk tube with a magnetic bar was charged with copper catalyst $(0.005 \mathrm{mmol}, 5 \mathrm{~mol} \%)$, ligand $(0.006 \mathrm{mmol}, 6$ $\mathrm{mol} \%$ ) and ${ }^{t} \mathrm{BuOLi}$ (16.0 mg, $0.2 \mathrm{mmol}, 2$ equiv.). The tube was capped with a septum. Under $\mathrm{N}_{2}$ atmosphere, solvent $(1 \mathrm{~mL}, 0.1 \mathrm{M})$ was added and the reaction mixture was stirred at room temperature for $1 \mathrm{~h}$. $\mathrm{B}_{2} \mathrm{Pin}_{2}(50.8 \mathrm{mg}, 0.2 \mathrm{mmol}, 2$ equiv.) and $\mathbf{1 a}(24.2$ $\mathrm{mg}, 0.1 \mathrm{mmol}$ ) were added and the resulting solution was stirred overnight at $15-20{ }^{\circ} \mathrm{C}$. Upon completion, the reaction mixture was quenched by saturated aq. $\mathrm{NH}_{4} \mathrm{Cl}(2 \mathrm{~mL})$ and extracted with ethyl acetate $(2 \mathrm{~mL} \times 3)$. The combined organic layers were dried over $\mathrm{Na}_{2} \mathrm{SO}_{4}$, and concentrated under vacuo. $\mathrm{CH}_{2} \mathrm{Br}_{2}(17.4 \mathrm{mg}, 0.1 \mathrm{mmol})$ as internal standard and $\mathrm{CDCl}_{3}$ were added to the residue. The yield of $\mathbf{2 a}$ and the ratio of $\mathbf{2 a} / \mathbf{2 a}$ ' was determined via ${ }^{1} \mathrm{H}$ NMR analysis. The ee value of $\mathbf{2 a}$ was determined via HPLC analysis after purification of the crude reaction mixture.

Table S1 screening results on ligand.<smiles>C=Cc1ccccc1CC1(C)C(=O)CCCC1=O</smiles>

$1 a$
$\mathrm{CuCl}(10 \mathrm{~mol} \%)$,

Ligand (12 mol\%)

$+\mathrm{B}_{2} \mathrm{Pin}_{2}$

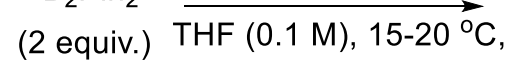
overnight, $\mathrm{N}_{2}$<smiles>O=C1CCC[C@@]2(O)[C@@H]1Cc1ccccc1[C@H]2Cc1ccccc1</smiles><smiles>O=C1CCCC23OB(O)CC2c2ccccc2CC13[103O]</smiles> 


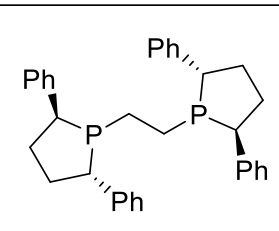

(S)-Ph-BPE

2a, $58 \%$ yield, $94 \%$ ee 2a', $17 \%$ yield<smiles>Pc1cccc(-c2c(P)ccc3c2OCO3)c1-c1c(P)ccc2c1OCO2</smiles>

(R)-SEGPhos

2a, $52 \%$ yield, $47 \%$ ee 2a', $22 \%$ yield

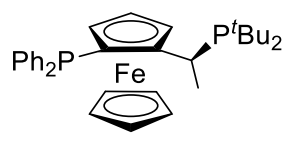

$(R, S)$

2a, 54\% yield, 55\% ee 2a', 23\% yield

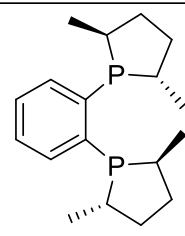

(S,S)-Me-DuPhos

2a, $39 \%$ yield, $23 \%$ ee

2a', $13 \%$ yield<smiles>CC(P)CC(C)Pc1ccccc1</smiles>

$(S, S)$

2a, $43 \%$ yield, $54 \%$ ee 2a', $27 \%$

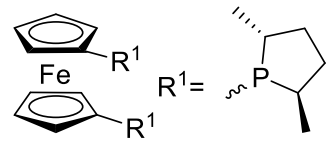

(R)

2a, $46 \%$ yield, $-34 \%$ ee 2a', $26 \%$ yield

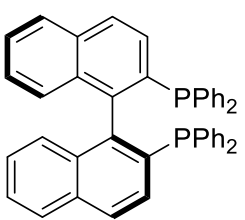

(S)-BINAP

2a, $41 \%$ yield, $-52 \%$ ee

2a', $25 \%$ yield

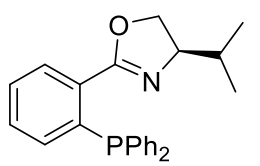

(R)-Phox

2a, $50 \%$ yield, $90 \%$ ee 2a', $32 \%$ yield

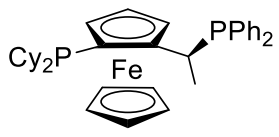

(R,S)

2a, $61 \%$ yield, $81 \%$ ee 2a', $17 \%$ yield<smiles>C[C@H](P)[C@H](C)P</smiles>

(S,S)-Chiraphos

2a, $37 \%$ yield, $94 \%$ ee

2a', $12 \%$ yield

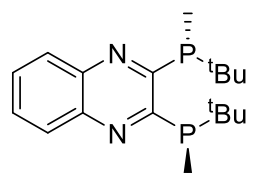

(R,R)-QuinoXP

2a, $28 \%$ yield, $26 \%$ ee

2a', $27 \%$ yield

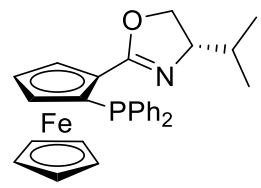

$(S, S)$

2a, $42 \%$ yield, $24 \%$ ee

2a', $36 \%$ yield

Table S2 screening results on catalyst.<smiles>C=Cc1ccccc1CC1(C)C(=O)CCCC1=O</smiles>

1a

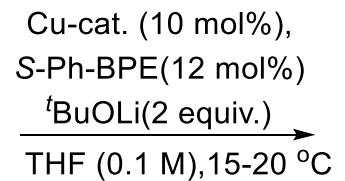

overnight, $\mathrm{N}_{2}$

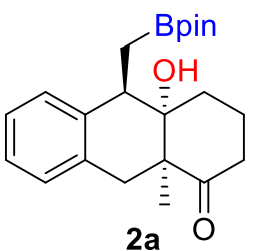

2a<smiles>[12CH3]C12Cc3ccccc3C3CB(O)CC31OCCCC2=O</smiles>

\begin{tabular}{cccc}
\hline $\mathrm{Cu}-\mathrm{cat}$. & $\mathbf{2 a}(\%)$ & $\boldsymbol{e e}(\%)$ & $\mathbf{2 a}^{\prime}(\%)$ \\
\hline $\mathrm{CuCl}$ & 58 & 94 & 17 \\
$\mathrm{CuI}$ & 63 & 64 & 11 \\
$\mathrm{CuBr}$ & 61 & 51 & 13 \\
$\mathrm{Cu}(\mathrm{OAc})_{2}$ & 58 & 90 & 11 \\
$\mathrm{Cu}\left(\mathrm{CH}_{3} \mathrm{CN}\right)_{4} \mathrm{PF}_{6}$ & 65 & 91 & 17 \\
$\mathrm{CuBr} 2$ & 46 & 42 & 9 \\
$\mathrm{Cu}(\mathrm{OTf})_{2}$ & 55 & 85 & 9 \\
\hline
\end{tabular}

Table S3 screening results on temperature.<smiles>C=Cc1ccccc1CC1(C)C(=O)CCCC1=O</smiles>

1a

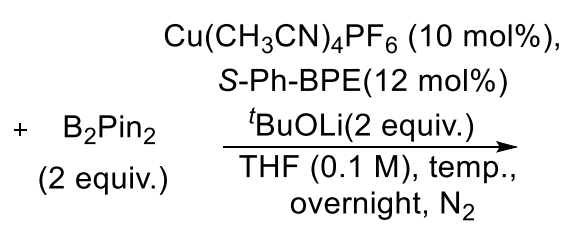

overnight, $\mathrm{N}_{2}$<smiles>C[C@]12Cc3ccccc3[C@@H](Cc3ccccc3)[C@]1(O)CCCC2=O</smiles><smiles>O=C1CCCC23OB(O)CC2c2ccccc2CC13[Te]</smiles>

S8 


\begin{tabular}{cccc}
\hline Temp. $\left({ }^{\circ} \mathrm{C}\right)$ & $\mathbf{2 a}(\%)$ & $\boldsymbol{e e}(\%)$ & $\mathbf{2 a} \boldsymbol{( \% )}$ \\
\hline 25 & 65 & 91 & 17 \\
-5 & 77 & 98 & 21 \\
0 & $82^{a}$ & 98 & 16 \\
0 & $84^{a, b}$ & 98 & 21 \\
\hline
\end{tabular}

[a] The reaction time was 4 h. [b] $\mathrm{Cu}\left(\mathrm{CH}_{3} \mathrm{CN}\right)_{4} \mathrm{PF}_{6}(5 \mathrm{~mol} \%), S$-Ph-BPE $(6 \mathrm{~mol} \%)$ were used.

Table S4 screening results on solvent.
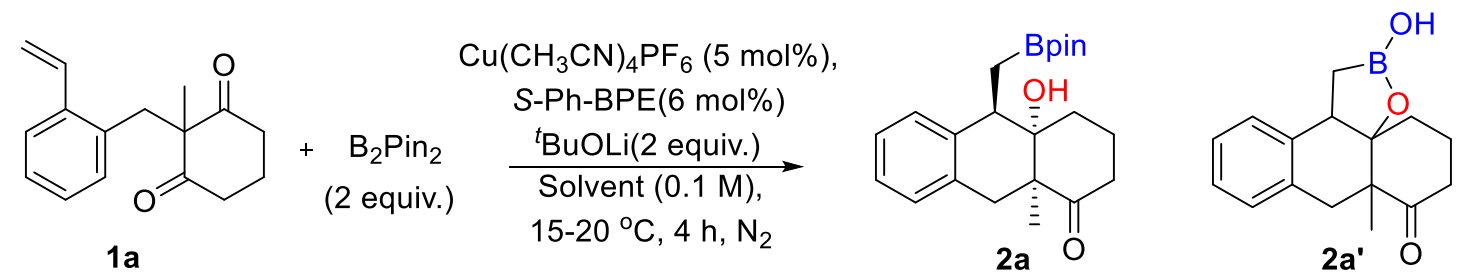

\begin{tabular}{cccc}
\hline Solvent & 2a (\%) & $\boldsymbol{e e}(\%)$ & $\mathbf{2 a} / \mathbf{2 a} \mathbf{~}$ \\
\hline $\mathrm{THF}\left(0^{\circ} \mathrm{C}\right)$ & 84 & 98 & $4 / 1$ \\
$\mathrm{TBME}$ & 23 & n.d. & $2.5 / 1$ \\
$\mathrm{Et}{ }_{2} \mathrm{O}$ & 52 & 97 & $>13 / 1$ \\
$\mathrm{MeCN}$ & 20 & n.d. & $11 / 1$ \\
$\mathrm{Tol}$ & 40 & n.d. & $4 / 1$ \\
$\mathrm{DMF}$ & 62 & n.d. & $1.7 / 1$ \\
$2-\mathrm{MeTHF}$ & 94 & 99 & $13 / 1$ \\
$\mathrm{THF} / \mathrm{Et} 2 \mathrm{O}=1 / 1\left(0^{\circ} \mathrm{C}, 6 \mathrm{~h}\right)$ & 80 & 95 & $8 / 1$ \\
$\mathrm{THF} / \mathrm{Et}{ }_{2} \mathrm{O}=1 / 2$ & 84 & 97 & $8.4 / 1$ \\
\hline
\end{tabular}

Table S5 screening results on temperature.

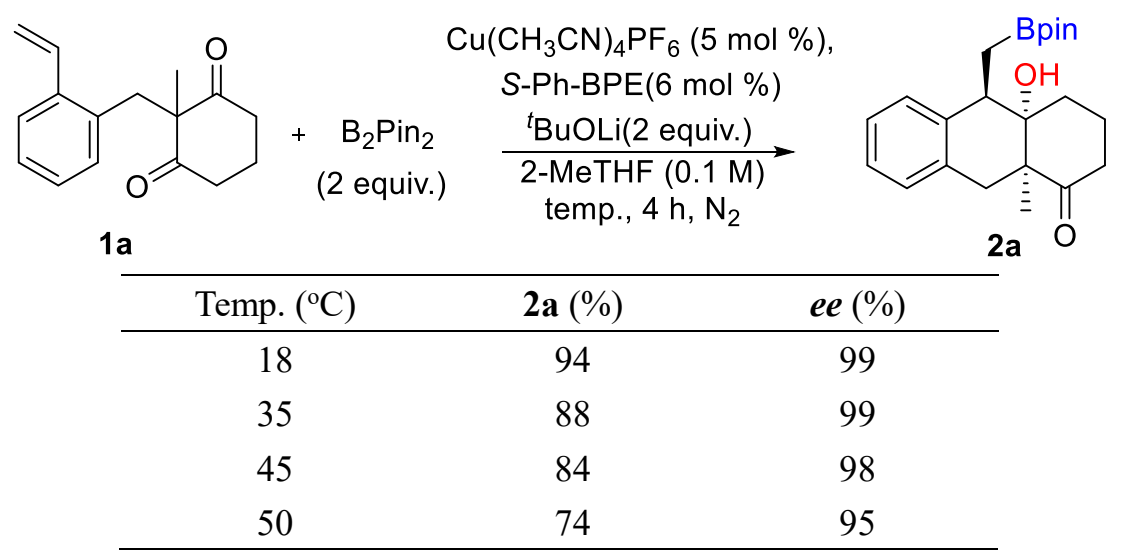

4. General Procedure for Catalytic Asymmetric Cyclization of 1,3-

\section{Diketones}

\subsection{General Procedure for Catalytic Asymmetric Cyclization of 1,3-Diketones}



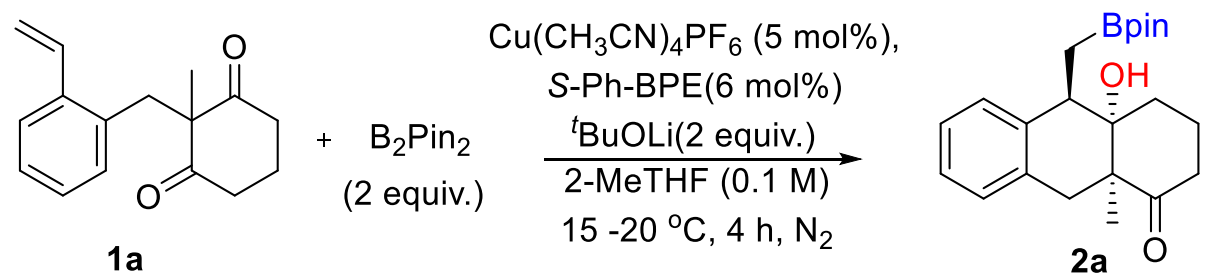

In the nitrogen filled glovebox, A $10 \mathrm{~mL}$ oven-dried Schlenk tube with a magnetic bar was charged with $\mathrm{Cu}\left(\mathrm{CH}_{3} \mathrm{CN}\right)_{4} \mathrm{PF}_{6}(3.7 \mathrm{mg}, 0.01 \mathrm{mmol}, 5 \mathrm{~mol} \%), S$-Ph-BPE (6.1 $\mathrm{mg}, 0.012 \mathrm{mmol}, 6 \mathrm{~mol} \%$ ) and ${ }^{t} \mathrm{BuOLi}$ ( $32.0 \mathrm{mg}, 0.4 \mathrm{mmol}, 2$ equiv.). The tube was capped with a septum. Under $\mathrm{N}_{2}$ atmosphere, 2-MeTHF $(2 \mathrm{~mL}, 0.1 \mathrm{M})$ was added and the reaction mixture was stirred at room temperature for $1 \mathrm{~h}$. $\mathrm{B}_{2} \mathrm{Pin}_{2}(102.0 \mathrm{mg}, 0.4$ mmol, 2 equiv.) and $\mathbf{1 a}(48.4 \mathrm{mg}, 0.2 \mathrm{mmol})$ were added and the resulting solution was stirred for $4 \mathrm{~h}$ at $15-20{ }^{\circ} \mathrm{C}$. Upon completion, the reaction mixture was quenched by saturated aq. $\mathrm{NH}_{4} \mathrm{Cl}(2 \mathrm{~mL})$ and extracted with ethyl acetate $(2 \mathrm{~mL} \times 3)$. The combined organic layers were dried over $\mathrm{Na}_{2} \mathrm{SO}_{4}$, and concentrated under vacuo. The residue was purified by silica gel column chromatography with a gradient eluent of petroleum ether/ethyl acetate affording the product. The ee value of $\mathbf{2 a}$ was determined via HPLC analysis.

On 1 mmol scale:

In the nitrogen filled glovebox, A $50 \mathrm{~mL}$ oven-dried Schlenk tube with a magnetic bar was charged with $\mathrm{Cu}\left(\mathrm{CH}_{3} \mathrm{CN}\right)_{4} \mathrm{PF}_{6}(18.5 \mathrm{mg}, 0.05 \mathrm{mmol}, 5 \mathrm{~mol} \%), S$-Ph-BPE (30.1 $\mathrm{mg}, 0.06 \mathrm{mmol}, 6 \mathrm{~mol} \mathrm{\%}$ ) and ${ }^{t} \mathrm{BuOLi}$ ( $160.0 \mathrm{mg}, 2.0 \mathrm{mmol}, 2$ equiv.). The tube was capped with a septum. Under $\mathrm{N}_{2}$ atmosphere, 2-MeTHF $(10 \mathrm{~mL}, 0.1 \mathrm{M})$ was added and the reaction mixture was stirred at room temperature for $1 \mathrm{~h}$. $\mathrm{B}_{2} \mathrm{Pin}_{2}(508.0 \mathrm{mg}, 2.0$ mmol, 2 equiv.) and $1 \mathbf{a}(242.0 \mathrm{mg}, 1 \mathrm{mmol})$ were added and the resulting solution was stirred for $4 \mathrm{~h}$ at $15-20{ }^{\circ} \mathrm{C}$. Upon completion, the reaction mixture was quenched by saturated aq. $\mathrm{NH}_{4} \mathrm{Cl}(10 \mathrm{~mL})$ and extracted with ethyl acetate $(5 \mathrm{~mL} \times 3)$. The combined organic layers were dried over $\mathrm{Na}_{2} \mathrm{SO}_{4}$, and concentrated under vacuo. The residue was purified by silica gel column chromatography with a gradient eluent of petroleum ether/ethyl acetate affording the product 2a. The ee value of $2 \mathbf{a}$ was determined via HPLC analysis (307 mg, 83\% yield, 99\% ee).

\subsection{General Procedure for Catalytic Asymmetric Cyclization of 1,3-Diketones (4d,} 4e)
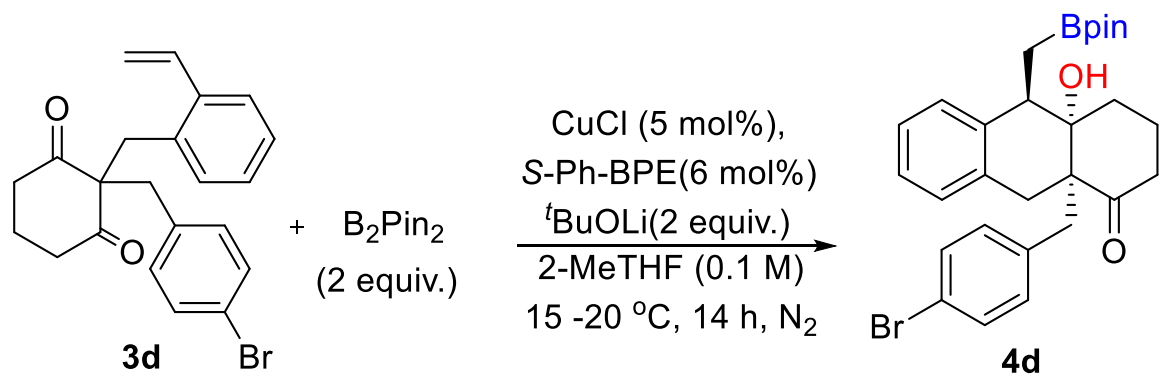

In the nitrogen filled glovebox, A $10 \mathrm{~mL}$ oven-dried Schlenk tube with a magnetic bar was charged with $\mathrm{CuCl}$ (1.0 mg, $0.01 \mathrm{mmol}, 5 \mathrm{mo}$ 1\%), $S$-Ph-BPE (6.1 mg, 0.012 
mmol, $6 \mathrm{~mol} \mathrm{\%}$ ) and ${ }^{t} \mathrm{BuOLi}(32.0 \mathrm{mg}, 0.4 \mathrm{mmol}, 2$ equiv.). The tube was capped with a septum. Under $\mathrm{N}_{2}$ atmosphere, 2-MeTHF $(2 \mathrm{~mL}, 0.1 \mathrm{M})$ was added and the reaction mixture was stirred at room temperature for $1 \mathrm{~h}$. $\mathrm{B}_{2} \mathrm{Pin}_{2}$ (102.0 mg, $0.4 \mathrm{mmol}, 2$ equiv.) and $3 \mathbf{d}(79.2 \mathrm{mg}, 0.2 \mathrm{mmol})$ were added and the resulting solution was stirred for $14 \mathrm{~h}$. Upon completion, the reaction mixture was quenched by saturated aq. $\mathrm{NH}_{4} \mathrm{Cl}(2 \mathrm{~mL})$ and extracted with ethyl acetate $(2 \mathrm{~mL} \times 3)$. The combined organic layers were dried over $\mathrm{Na}_{2} \mathrm{SO}_{4}$, and concentrated under vacuo. The residue was purified by silica gel column chromatography with a gradient eluent of petroleum ether/ethyl acetate affording the product. The ee value of $\mathbf{4 d}$ was determined via HPLC analysis.

\subsection{General Procedure for Catalytic Asymmetric Cyclization of 1,3-Diketones (6b)}<smiles>CC1(C/C=C/c2ccccc2)C(=O)CCCC1=O</smiles>

$5 b$

$$
\begin{array}{cc}
\mathrm{Cu}\left(\mathrm{CH}_{3} \mathrm{CN}\right)_{4} \mathrm{PF}_{6}(5 \mathrm{~mol} \%), \\
(R, R)-Q \text { QuinoXP }(6 \mathrm{~mol} \%)
\end{array}
$$

In the nitrogen filled glovebox, A $10 \mathrm{~mL}$ oven-dried Schlenk tube with a magnetic bar was charged with $\mathrm{CuCl}(1.0 \mathrm{mg}, 0.01 \mathrm{mmol}, 5 \mathrm{~mol} \%),(R, R)$-QunioXP (4.0 mg, $0.012 \mathrm{mmol}, 6 \mathrm{~mol} \%$ ) and ${ }^{t} \mathrm{BuOLi}(32.0 \mathrm{mg}, 0.4 \mathrm{mmol}, 2$ equiv.). The tube was capped with a septum. Under $\mathrm{N}_{2}$ atmosphere, 2-MeTHF (2 mL, $0.1 \mathrm{M}$ ) was added and the reaction mixture was stirred at room temperature for $1 \mathrm{~h}$. $\mathrm{B}_{2} \mathrm{Pin}_{2}(102.0 \mathrm{mg}, 0.4 \mathrm{mmol}$, 2 equiv.) and $\mathbf{5 b}$ (48.4 $\mathrm{mg}, 0.2 \mathrm{mmol}$ ) were added and the resulting solution was stirred for $3 \mathrm{~h}$ at $25^{\circ} \mathrm{C}$. Upon completion, the reaction mixture was quenched by saturated aq. $\mathrm{NH}_{4} \mathrm{Cl}(2 \mathrm{~mL})$ and extracted with ethyl acetate $(2 \mathrm{~mL} \times 3)$. The combined organic layers were dried over $\mathrm{Na}_{2} \mathrm{SO}_{4}$, and concentrated under vacuo. The residue was purified by silica gel column chromatography with a gradient eluent of petroleum ether/ethyl acetate affording the product. The ratio of isomers was determined via ${ }^{1} \mathrm{H} N \mathrm{NR}$ analysis of the crude reaction mixture and the ee value of $\mathbf{6} \mathbf{b}$ was determined via HPLC analysis after purification.

The corresponding racemate was obtained using the same procedure with dppbz as the race ligand.

\section{The functional-group compatibility study}

\section{General Procedure for the functional-group compatibility study}

In the nitrogen filled glovebox, A $10 \mathrm{~mL}$ oven-dried Schlenk tube with a magnetic bar was charged with $\mathrm{Cu}\left(\mathrm{CH}_{3} \mathrm{CN}\right)_{4} \mathrm{PF}_{6}(0.9 \mathrm{mg}, 0.0025 \mathrm{mmol}, 5 \mathrm{~mol} \%), S$-Ph-BPE ( $1.5 \mathrm{mg}, 0.0030 \mathrm{mmol}, 6 \mathrm{~mol} \%$ ) and ${ }^{t} \mathrm{BuOLi}(8.0 \mathrm{mg}, 0.1 \mathrm{mmol}, 2$ equiv.). The tube was capped with a septum. Under $\mathrm{N}_{2}$ atmosphere, 2-MeTHF $(0.5 \mathrm{~mL}, 0.1 \mathrm{M})$ was added and the reaction mixture was stirred at room temperature for $1 \mathrm{~h}$. $\mathrm{B}_{2} \mathrm{Pin}_{2}(25.4 \mathrm{mg}, 0.1$ mmol, 2 equiv. $), 1 a(12.1 \mathrm{mg}, 0.05 \mathrm{mmol})$ and additive $(0.05 \mathrm{mmol})$ were added and the resulting solution was stirred for $4 \mathrm{~h}$. Upon completion, the reaction mixture was 
quenched by saturated aq. $\mathrm{NH}_{4} \mathrm{Cl}(1 \mathrm{~mL})$, the reaction mixture was quenched by saturated aq. $\mathrm{NH}_{4} \mathrm{Cl}(2 \mathrm{~mL})$ and extracted with ethyl acetate $(2 \mathrm{~mL} \times 3)$. The combined organic layers were dried over $\mathrm{Na}_{2} \mathrm{SO}_{4}$, and concentrated under vacuo. $\mathrm{CH}_{2} \mathrm{Br}_{2}$ (17.4 $\mathrm{mg}, 0.1 \mathrm{mmol}$ ) as internal standard and $\mathrm{CDCl}_{3}$ were added to the residue. The yield of 2a and the recovery of additive were determined via ${ }^{1} \mathrm{H}$ NMR analysis or Gas Chromatography. The ee value of 2a was determined via HPLC analysis after purification of the crude reaction mixture.

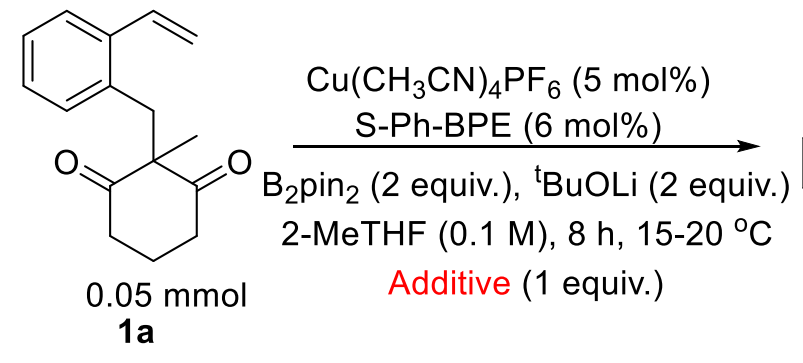

Yield $>85 \%$, ee $>95 \%$; Recovery of additive $>90 \%$<smiles>CC12Cc3ccccc3C(CBr)C1(O)CCCC2=O</smiles>

no additive $94 \%$ yield $99 \%$ ee<smiles>CNC(=O)c1ccccc1</smiles>

$88 \%$ yield, $99 \%$ ee $>99 \%$ recovery<smiles>CN(C)c1ccccc1</smiles>

$94 \%$ yield, $99 \%$ ee $>99 \%$ recovery<smiles>COCCCc1ccccc1</smiles>

$98 \%$ yield, $99 \%$ ee $>99 \%$ recovery<smiles>CCOC(=O)c1ccccc1</smiles>

$98 \%$ yield, $99 \%$ ee $>99 \%$ recovery<smiles></smiles>
$99 \%$ recovery<smiles>CC1(C)OCC(c2ccccc2)O1</smiles>
$90 \%$ recovery<smiles>[Se-]c1ccccc1</smiles>

$87 \%$ yield, $99 \%$ ee $>96 \%$ recovery<smiles>CSc1ccccc1</smiles>

$98 \%$ yield, $99 \%$ ee $>99 \%$ recovery<smiles>[SeH2]CCCc1ccccc1</smiles>

$96 \%$ yield, $99 \%$ ee $>99 \%$ recovery 


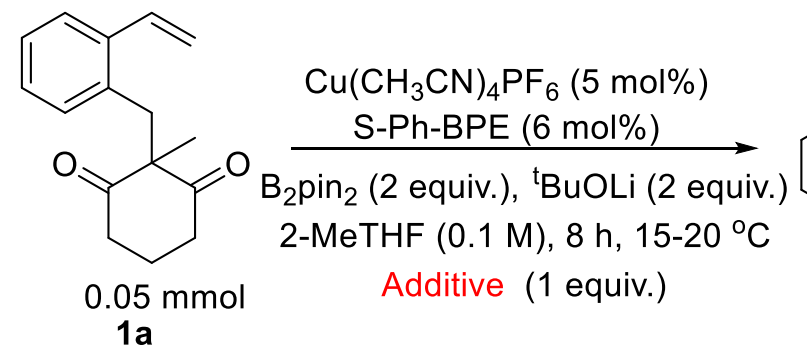

Yield $<85 \%$, ee $>95 \%$ or Recovery of additive $<90 \%$<smiles>CC12Cc3ccccc3C(CBr)C1(O)CCCC2=O</smiles>

no additive $94 \%$ yield $99 \%$ ee<smiles>COc1ccc(I)cc1</smiles>

$88 \%$ yield, $99 \%$ ee $79 \%$ recovery<smiles>c1ccc2occc2c1</smiles>

$85 \%$ yield, $99 \%$ ee $84 \%$ recovery<smiles>BrC1CCCCC1</smiles>
$96 \%$ yield, $99 \%$ ee $45 \%$ recovery<smiles>CCOC(=O)C(=O)c1ccccc1</smiles>

$73 \%$ yield, $99 \%$ ee $69 \%$ recovery

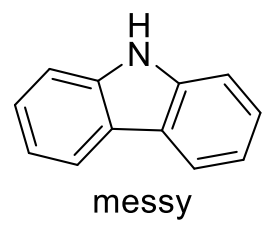<smiles>N#Cc1ccccc1</smiles>

$99 \%$ yield, $99 \%$ ee $86 \%$ recovery<smiles>Cn1ccc2ccccc21</smiles>

$68 \%$ yield, $99 \%$ ee $76 \%$ recovery

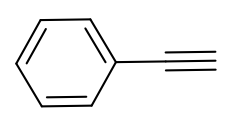

$68 \%$ yield, $99 \%$ ee $69 \%$ recovery<smiles>C=CCc1ccccc1</smiles>

$94 \%$ yield, $99 \%$ ee $38 \%$ recovery<smiles>O=[N+]([O-])c1ccccc1</smiles>

$68 \%$ yield, $99 \%$ ee $73 \%$ recovery<smiles>O=Cc1ccc2ccccc2c1</smiles>

$66 \%$ yield $31 \%$ recovery $61 \%$ recovery<smiles></smiles>

\section{General procedures for the transformations of the enantioenriched} cyclic boronic ester products.

\subsection{General procedure for synthesis of $7 \mathbf{a}^{\mathrm{S} 7}$}<smiles>C[C@]12Cc3ccccc3[C@H](CBr)[C@]1(O)CCCC2=O</smiles><smiles>O=C(OO)[N+](=O)O</smiles>

$2 a$<smiles>CC1=CCC[C@@]2(O)[C@@H](CO)c3ccccc3C[C@]12C</smiles>

$84 \%$ yield, $98 \%$ ee
To a stirred solution of $\mathbf{2 a}(74.0 \mathrm{mg}, 0.2 \mathrm{mmol})$ in THF $(2 \mathrm{~mL})$ was added a 
suspension of $\mathrm{NaOH}(3 \mathrm{M}, 0.34 \mathrm{~mL})$ at $0{ }^{\circ} \mathrm{C}$. Then $30 \%$ aqueous $\mathrm{H}_{2} \mathrm{O}_{2}(0.22 \mathrm{~mL})$ was added dropwise. The mixture was stirred for $1 \mathrm{~h}$. The reaction was diluted with water and extracted with ethyl acetate three times. The combined organics were dried over $\mathrm{Na}_{2} \mathrm{SO}_{4}$ and concentrated in vacuo. The residue was purified by silica gel column chromatography with a gradient eluent of petroleum ether/ethyl acetate affording $\mathbf{7 a}$ in $84 \%$ yield. The ee value of $7 \mathbf{a}$ was determined via HPLC analysis.

\subsection{Gerneral procedure for synthesis of $7 b^{S 8}$}<smiles>[R9]C[C@@H]1c2ccccc2C[C@]2(C)C(=O)CCC[C@]12O</smiles>

$2 a$

$$
\underset{\mathrm{MeOH}, \mathrm{rt}}{\stackrel{\mathrm{KHF}_{2}(4.5 \mathrm{M})}{\longrightarrow}}
$$<smiles>CC(C)(C)CBr</smiles>
$76 \%$ yield

To a stirred solution of $2 \mathrm{a}(74.0 \mathrm{mg}, 0.2 \mathrm{mmol})$ in $\mathrm{MeOH}(1 \mathrm{~mL})$ was added $4.5 \mathrm{M}$ aqueous $\mathrm{KHF}_{2}(0.22 \mathrm{~mL}$, 5equiv.) and the reaction mixture was stirred for $2 \mathrm{~h}$ at room temperature. The solvent was removed under reduced pressure. The residue was dissolved in toluene $(0.5 \mathrm{~mL})$ and concentrated in vacuo for three times to remove the residual water and pinacol. Then the crude mixture was dissolved in acetone and filtered. The combined organics were concentrated in vacuo. The pale solid obtained was dissolved in a minimum amount of acetone and then hexanes were added to the solution. The participates were collected and dried in vacuo to obtained $\mathbf{7 b}$ as a white solid in $76 \%$ yield.

\subsection{General procedure for synthesis of $7 \mathrm{c}^{\mathrm{S9}}$}
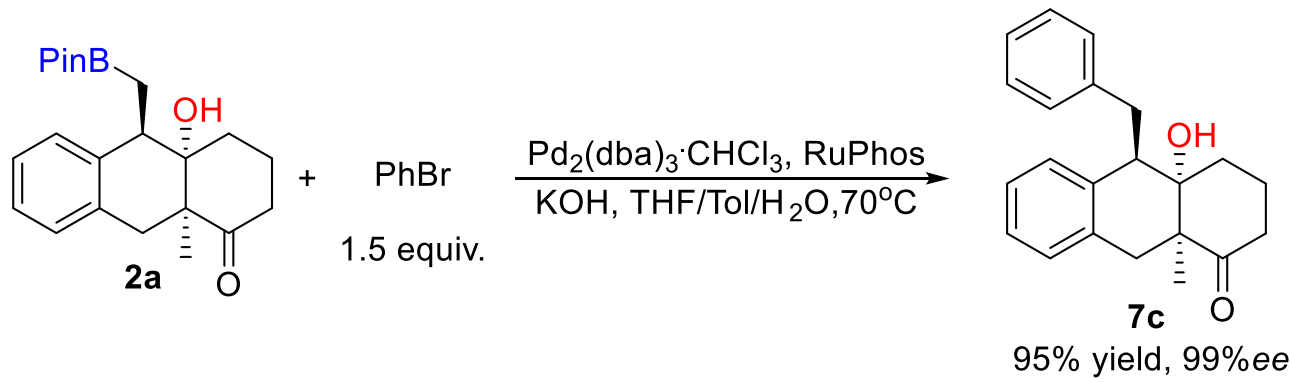

In the nitrogen filled glovebox, A $10 \mathrm{~mL}$ oven-dried Schlenk tube with a magnetic bar was charged with $\mathrm{Pd}_{2}(\mathrm{dba})_{3} \cdot \mathrm{CHCl}_{3}(4.1 \mathrm{mg}, 0.004 \mathrm{mmol}, 2 \mathrm{~mol} \%)$ and RuPhos (3.7 $\mathrm{mg}, 0.008 \mathrm{mmol}, 4 \mathrm{~mol} \%)$. The tube was capped with a septum and removed from the glovebox. Under $\mathrm{N}_{2}$ atmosphere, $\mathrm{KOH}$ (33.6 mg, $0.6 \mathrm{mmol}, 3$ equiv.), THF (0.5 mL) and Toluene $(1 \mathrm{~mL})$ was added and the reaction mixture was stirred at room temperature for $30 \mathrm{~min}$. A solution of $\mathbf{2 a}(74.0 \mathrm{mg}, 0.2 \mathrm{mmol})$ in THF $(0.5 \mathrm{~mL})$ was added followed by addition of bromobenzene (37.7 $\mathrm{mg}, 0.24 \mathrm{mmol}, 1.2$ equiv.). Then the degassed water (sparged with $\mathrm{N}_{2}$ for $2 \mathrm{~h}, 0.2 \mathrm{~mL}$ ) was added. the reaction mixture was heated to

\footnotetext{
S8 Burns A. R.; Gonzalez J. S.; Lam H. W. Angew. Chem. Int. Ed. 2012, 51, 10827.

s9 Blaisdell T. P.; Morken J. P. J. Am. Chem. Soc. 2015, 137, 8712.
} 
$70{ }^{\circ} \mathrm{C}$ and stirred overnight. The mixture was allowed to cool to room temperature and quenched with water. The layers were allowed to separated and the aqueous layer was extracted with ethyl acetate three times. The combined organics were dried over $\mathrm{Na}_{2} \mathrm{SO}_{4}$ and concentrated under reduced pressure. The residue was purified by silica gel column chromatography with a gradient eluent of petroleum ether/ethyl acetate affording $\mathbf{7} \mathbf{c}$ in $95 \%$ yield. The ee value of $\mathbf{7} \mathbf{c}$ was determined via HPLC analysis.

\subsection{General procedure for synthesis of $7 \mathbf{d}^{\mathrm{S} 9}$}<smiles>C[C@]12Cc3ccccc3[C@@H](C[Pb])[C@@]1(O)CCCC2=O</smiles><smiles>[R16][R9]</smiles><smiles>CC(C)=CC[C@@H]1c2ccccc2CC2(O)C(=O)CCC[C@]12O</smiles>

$91 \%$ yield, $97 \%$ ee

In the nitrogen filled glovebox, A $10 \mathrm{~mL}$ oven-dried Schlenk tube with a magnetic bar was charged with $\mathrm{Pd}_{2}(\mathrm{dba})_{3} \cdot \mathrm{CHCl}_{3}(4.1 \mathrm{mg}, 0.004 \mathrm{mmol}, 2 \mathrm{~mol} \%)$ and RuPhos (3.7 $\mathrm{mg}, 0.008 \mathrm{mmol}, 4 \mathrm{~mol} \%)$. The tube was capped with a septum and removed from the glovebox. Under $\mathrm{N}_{2}$ atmosphere, $\mathrm{KOH}$ (33.6 mg, $0.6 \mathrm{mmol}, 3$ equiv.), THF (0.5 mL) and Toluene $(1 \mathrm{~mL})$ was added and the reaction mixture was stirred at room temperature for $30 \mathrm{~min}$. A solution of $2 \mathrm{a}(74.0 \mathrm{mg}, 0.2 \mathrm{mmol})$ in THF $(0.5 \mathrm{~mL})$ was added followed by addition of 1-bromo-2-methylprop-1-ene ( $32.2 \mathrm{mg}, 0.24 \mathrm{mmol}, 1.2$ equiv.). Then the degassed water (sparged with $\mathrm{N}_{2}$ for $2 \mathrm{~h}, 0.2 \mathrm{~mL}$ ) was added. the reaction mixture was heated to $70{ }^{\circ} \mathrm{C}$ and stirred overnight. The mixture was allowed to cool to room temperature and quenched with water. The layers were allowed to separated and the aqueous layer was extracted with ethyl acetate three times. The combined organics were dried over $\mathrm{Na}_{2} \mathrm{SO}_{4}$ and concentrated under reduced pressure. The residue was purified by silica gel column chromatography with a gradient eluent of petroleum ether/ethyl acetate affording $\mathbf{7 d}$ in $91 \%$ yield. The ee value of $\mathbf{7 d}$ was determined via HPLC analysis.

\section{The characterization of the new products}<smiles>C=Cc1ccccc1CC1(C)C(=O)CCCC1=O</smiles>

The title compound 1a was synthesized according to General Procedure (SI 2.1), and it was purified by column chromatography on silica gel.

${ }^{1} \mathrm{H}$ NMR (600 MHz, Chloroform- $d$ ) $\delta 7.47$ (d, $\left.J=7.6 \mathrm{~Hz}, 1 \mathrm{H}\right), 7.20$ (dd, $J=7.6,7.2$ $\mathrm{Hz}, 1 \mathrm{H}), 7.15$ (dd, $J=7.9,7.8 \mathrm{~Hz}, 1 \mathrm{H}), 6.98-6.90(\mathrm{~m}, 2 \mathrm{H}), 5.61(\mathrm{dd}, J=17.2,1.4 \mathrm{~Hz}$, $1 \mathrm{H}), 5.30(\mathrm{dd}, J=11.0,1.4 \mathrm{~Hz}, 1 \mathrm{H}), 3.21(\mathrm{~s}, 2 \mathrm{H}), 2.57-2.50(\mathrm{~m}, 2 \mathrm{H}), 2.38-2.28(\mathrm{~m}, 2 \mathrm{H})$, 1.76-1.68 (m, 1H), 1.67-1.59 (m, 1H), $1.29(\mathrm{~s}, 3 \mathrm{H}) ;{ }^{13} \mathrm{C}$ NMR (101 MHz, Chloroform- 
d) $\delta 211.4,137.5,134.6,133.9,130.6,127.7,127.5,126.3,116.5,64.9,40.6,39.5,21.5$, 16.7; HRMS: m/z (ESI) calculated $[\mathrm{M}+\mathrm{Na}]^{+}: 265.1204$, found: 265.1194 .<smiles>C=Cc1cc(C)ccc1CC1(C)C(=O)CCCC1=O</smiles>

$1 \mathrm{~b}$

The title compound $\mathbf{1 b}$ was synthesized according to General Procedure (SI 2.1), and it was purified by column chromatography on silica gel.

${ }^{1} \mathrm{H}$ NMR (400 MHz, Chloroform- $d$ ) $\delta 7.28(\mathrm{~s}, 1 \mathrm{H}), 7.05-6.62(\mathrm{~m}, 3 \mathrm{H}), 5.59$ (d, $J=17.2$ $\mathrm{Hz}, 1 \mathrm{H}), 5.28(\mathrm{~d}, J=11.0 \mathrm{~Hz}, 1 \mathrm{H}), 3.16(\mathrm{~s}, 2 \mathrm{H}), 2.67-2.44(\mathrm{~m}, 2 \mathrm{H}), 2.43-2.22(\mathrm{~m}, 5 \mathrm{H})$, 1.88-1.58 (m, 2H), $1.27(\mathrm{~s}, 3 \mathrm{H}) ;{ }^{13} \mathrm{C}$ NMR (101 MHz, Chloroform- $d$ ) $\delta 211.5,137.2$, 137.0, 134.6, 130.8, 130.5, 128.5, 126.8, 116.1, 65.1, 40.5, 39.5, 21.1, 21.0, 16.8; HRMS: m/z (ESI) calculated [M+H] $]^{+}: 257.1542$, found: 257.1537 .<smiles>C=Cc1cc(Cl)ccc1CC1(C)C(=O)CCCC1=O</smiles>

The title compound 1c was synthesized according to General Procedure (SI 2.1), and it was purified by column chromatography on silica gel.

${ }^{1} \mathrm{H}$ NMR (400 MHz, Chloroform- $d$ ) $\delta 7.44(\mathrm{~d}, J=2.3 \mathrm{~Hz}, 1 \mathrm{H}), 7.12$ (dd, $J=8.4,2.3$ $\mathrm{Hz}, 1 \mathrm{H}), 7.05-6.84(\mathrm{~m}, 2 \mathrm{H}), 5.62(\mathrm{~d}, J=17.2 \mathrm{~Hz}, 1 \mathrm{H}), 5.36(\mathrm{~d}, J=11.0 \mathrm{~Hz}, 1 \mathrm{H}), 3.17$ (s, 2H), 2.63-2.52 (m, 2H), 2.41-2.28 (m, 2H), 1.87-1.70 (m, 2H), $1.29(\mathrm{~s}, 3 \mathrm{H}) ;{ }^{13} \mathrm{C}$ NMR (101 MHz, Chloroform-d) $\delta$ 211.1, 139.3, 133.7, 133.2, 132.6, 131.9, 127.6, 126.3, 117.7, 64.8, 39.3, 39.1, 22.0, 16.7; HRMS: m/z (ESI) calculated $[\mathrm{M}+\mathrm{Na}]^{+}: 299.0815$, found: 299.0808 .<smiles>C=Cc1cc(F)ccc1CC1(C)C(=O)CCCC1=O</smiles>

The title compound 1d was synthesized according to General Procedure (SI 2.1), and it was purified by column chromatography on silica gel.

${ }^{1} \mathrm{H}$ NMR (400 MHz, Chloroform- $d$ ) $\delta$ 7.20-7.12 (m, 1H), 6.99-6.88 (m, 2H), 6.88-6.82 $(\mathrm{m}, 1 \mathrm{H}), 5.61(\mathrm{~d}, J=17.2 \mathrm{~Hz}, 1 \mathrm{H}), 5.36(\mathrm{~d}, J=11.0 \mathrm{~Hz}, 1 \mathrm{H}), 3.18(\mathrm{~s}, 2 \mathrm{H}), 2.61-2.51$ $(\mathrm{m}, 2 \mathrm{H}), 2.38-2.28(\mathrm{~m}, 2 \mathrm{H}), 1.84-172(\mathrm{~m}, 1 \mathrm{H}), 1.69-1.57(\mathrm{~m}, 1 \mathrm{H}), 1.28(\mathrm{~s}, 3 \mathrm{H}) ;{ }^{13} \mathrm{C}$ NMR (101 MHz, Chloroform- $d$ ) $\delta 211.3,161.9(\mathrm{~d}, J=245.9 \mathrm{~Hz}), 139.6(\mathrm{~d}, J=7.5 \mathrm{~Hz})$, $133.8,132.2(\mathrm{~d}, J=8.1 \mathrm{~Hz}), 129.9(\mathrm{~d}, J=3.0 \mathrm{~Hz}), 117.5,114.6(\mathrm{~d}, J=21.3 \mathrm{~Hz}), 112.6$ $(\mathrm{d}, J=21.7 \mathrm{~Hz}), 64.8,39.4,21.8,16.7 ; \mathrm{HRMS}: \mathrm{m} / \mathrm{z}(\mathrm{ESI})$ calculated $[\mathrm{M}+\mathrm{Na}]^{+}: 283.1110$, found:283.1105. 
<smiles>C=Cc1ccc(F)cc1CC1(C)C(=O)CCCC1=O</smiles>

The title compound 1e was synthesized according to General Procedure (SI 2.1), and it was purified by column chromatography on silica gel.

${ }^{1} \mathrm{H}$ NMR (400 MHz, Chloroform-d) $\delta$ 7.50-7.39 (m, 1H), 6.98-6.85 (m, 2H), 6.67 (d, $J$ $=10.0 \mathrm{~Hz}, 1 \mathrm{H}), 5.54(\mathrm{~d}, J=17.2 \mathrm{~Hz}, 1 \mathrm{H}), 5.30(\mathrm{~d}, J=11.0 \mathrm{~Hz}, 1 \mathrm{H}), 3.20(\mathrm{~s}, 2 \mathrm{H}), 2.66-$ $2.52(\mathrm{~m}, 2 \mathrm{H}), 2.46-2.28(\mathrm{~m}, 2 \mathrm{H}), 1.88-1.64(\mathrm{~m}, 2 \mathrm{H}), 1.31(\mathrm{~s}, 3 \mathrm{H}) ;{ }^{13} \mathrm{C} \mathrm{NMR}(101 \mathrm{MHz}$, Chloroform- $d$ ) $\delta 210.9,161.9(\mathrm{~d}, J=247.4 \mathrm{~Hz}), 136.4(\mathrm{~d}, J=7.3 \mathrm{~Hz}), 133.82(\mathrm{~d}, J=$ $4.8 \mathrm{~Hz}), 133.79,128.1$ (d, $J=8.2 \mathrm{~Hz}), 116.8$ (d, $J=21.5 \mathrm{~Hz}), 116.3,114.4$ (d, $J=21.2$ $\mathrm{Hz}), 64.7,39.2,39.1,22.3,16.7$; HRMS: $\mathrm{m} / \mathrm{z}$ (ESI) calculated $[\mathrm{M}+\mathrm{H}]^{+}: 261.1291$, found:261.1288.<smiles>C=Cc1ccc(OC)cc1CC1(C)C(=O)CCCC1=O</smiles>

The title compound 1f was synthesized according to General Procedure (SI 2.1), and it was purified by column chromatography on silica gel.

${ }^{1} \mathrm{H}$ NMR (400 MHz, Chloroform- $d$ ) $\delta 7.34$ (d, $\left.J=8.6 \mathrm{~Hz}, 1 \mathrm{H}\right), 6.77$ (dd, $J=17.2,11.0$ $\mathrm{Hz}, 1 \mathrm{H}), 6.68(\mathrm{~d}, J=8.6 \mathrm{~Hz}, 1 \mathrm{H}), 6.41(\mathrm{~s}, 1 \mathrm{H}), 5.42(\mathrm{~d}, J=17.2 \mathrm{~Hz}, 1 \mathrm{H}), 5.11(\mathrm{~d}, J=$ $11.0 \mathrm{~Hz}, 1 \mathrm{H}), 3.67(\mathrm{~s}, 3 \mathrm{H}), 3.10(\mathrm{~s}, 2 \mathrm{H}), 2.52-2.42(\mathrm{~m}, 2 \mathrm{H}), 2.35-2.21(\mathrm{~m}, 2 \mathrm{H}), 1.71-$ $1.62(\mathrm{~m}, 1 \mathrm{H}), 1.59-1.52(\mathrm{~m}, 1 \mathrm{H}), 1.22(\mathrm{~s}, 3 \mathrm{H}) ;{ }^{13} \mathrm{C}$ NMR (101 MHz, Chloroform-d $) \delta$ 211.3, 158.9, 135.3, 133.8, 130.0, 127.4, 115.4, 114.3, 113.4, 64.7, 55.1, 40.5, 39.4, 21.6, 16.7; HRMS: m/z (ESI) calculated $[\mathrm{M}+\mathrm{Na}]^{+}: 295.1310$, found:295.1302.<smiles>C=Cc1ccc(C(F)(F)F)cc1CC1(C)C(=O)CCCC1=O</smiles>

The title compound $\mathbf{1 g}$ was synthesized according to General Procedure (SI 2.1), and it was purified by column chromatography on silica gel.

${ }^{1} \mathrm{H}$ NMR $(600 \mathrm{MHz}$, Chloroform- $d$ ) $\delta 7.56(\mathrm{~d}, J=8.1 \mathrm{~Hz}, 1 \mathrm{H}), 7.44(\mathrm{~d}, J=8.1 \mathrm{~Hz}, 1 \mathrm{H})$, $7.18(\mathrm{~s}, 1 \mathrm{H}), 7.03(\mathrm{dd}, J=17.2,11.0 \mathrm{~Hz}, 1 \mathrm{H}), 5.68(\mathrm{~d}, J=17.2 \mathrm{~Hz}, 1 \mathrm{H}), 5.44(\mathrm{~d}, J=$ $11.0 \mathrm{~Hz}, 1 \mathrm{H}), 3.28(\mathrm{~s}, 2 \mathrm{H}), 2.69-2.54(\mathrm{~m}, 2 \mathrm{H}), 2.43-2.30(\mathrm{~m}, 2 \mathrm{H}), 1.86(\mathrm{~m}, 1 \mathrm{H}), 1.66$ $(\mathrm{m}, 1 \mathrm{H}), 1.33(\mathrm{~s}, 3 \mathrm{H}) ;{ }^{13} \mathrm{C}$ NMR $(101 \mathrm{MHz}$, Chloroform- $d$ ) $\delta 210.8,141.3,135.1$, $133.9,129.5$ (q, $J=32.4 \mathrm{~Hz}), 126.9,126.9$ (q, $J=3.8 \mathrm{~Hz}), 124.0$ (q, $J=272.1 \mathrm{~Hz})$, 123.9 (q, $J=3.7 \mathrm{~Hz}), 118.7,64.7,39.0,38.3,22.9,16.7$; HRMS: m/z (ESI) calculated $[\mathrm{M}+\mathrm{Na}]^{+}: 333.1078$, found:333.1067. 
<smiles>C=Cc1cc(OC)ccc1CC1(C)C(=O)CCCC1=O</smiles>

The title compound $\mathbf{1 h}$ was synthesized according to General Procedure (SI 2.1), and it was purified by column chromatography on silica gel.

${ }^{1} \mathrm{H}$ NMR (400 MHz, Chloroform- $d$ ) $\delta 6.99(\mathrm{~d}, J=2.5 \mathrm{~Hz}, 1 \mathrm{H}), 6.96-6.84(\mathrm{~m}, 2 \mathrm{H}), 6.72$ $(\mathrm{dd}, J=8.5,2.5 \mathrm{~Hz}, 1 \mathrm{H}), 5.61(\mathrm{~d}, J=17.2 \mathrm{~Hz}, 1 \mathrm{H}), 5.31(\mathrm{~d}, J=11.0 \mathrm{~Hz}, 1 \mathrm{H}), 3.80(\mathrm{~s}$, $1 \mathrm{H}), 3.14(\mathrm{~s}, 1 \mathrm{H}), 2.58-2.48(\mathrm{~m}, 2 \mathrm{H}), 2.38-2.26(\mathrm{~m}, 2 \mathrm{H}), 1.76-1.58(\mathrm{~m}, 2 \mathrm{H}), 1.27(\mathrm{~s}$, $3 \mathrm{H}) ;{ }^{13} \mathrm{C}$ NMR (101 MHz, Chloroform-d) $\delta 211.7,158.6,138.6,134.5,131.8,126.2$, 116.5, 113.5, 111.0, 65.0, 55.1, 40.3, 39.5, 21.1, 16.7; HRMS: m/z (ESI) calculated $[\mathrm{M}+\mathrm{Na}]^{+}: 395.1310$, found:395.1305.<smiles>C=C(C)c1ccccc1CC1(C)C(=O)CCCC1=O</smiles>

The title compound 1i was synthesized according to General Procedure (SI 2.2), and it was purified by column chromatography on silica gel.

${ }^{1} \mathrm{H}$ NMR (400 MHz, Chloroform- $d$ ) $\delta$ 7.22-7.04 (m, 3H), 6.91 (d, $\left.J=7.2 \mathrm{~Hz}, 1 \mathrm{H}\right), 5.23$ (s, 1H), $4.83(\mathrm{~s}, 1 \mathrm{H}), 3.29(\mathrm{~s}, 3 \mathrm{H}), 2.83-2.49(\mathrm{~m}, 3 \mathrm{H}), 2.04(\mathrm{~s}, 3 \mathrm{H}), 1.97-1.87(\mathrm{~m}, 1 \mathrm{H})$, 1.86-1.76 (m, 1H), 1.19 (s, 3H); ${ }^{13} \mathrm{C}$ NMR (101 MHz, Chloroform-d) $\delta 210.5,144.8$, $144.5,132.8,129.5,128.4,126.6,126.6,116.2,65.9,39.3,38.6,25.1,20.2,17.4$; HRMS: $\mathrm{m} / \mathrm{z}$ (ESI) calculated $[\mathrm{M}+\mathrm{H}]^{+}: 257.1542$, found:257.1539.<smiles>C=Cc1ccccc1CC1(Cc2ccccc2)C(=O)CCCC1=O</smiles>

The title compound 3a was synthesized according to General Procedure (SI 2.3), and it was purified by column chromatography on silica gel.

${ }^{1} \mathrm{H}$ NMR (400 MHz, Chloroform- $d$ ) $\delta$ 7.50-7.44 (m, 1H), 7.23-7.12 (m, 5H), 7.06-6.95 $(\mathrm{m}, 4 \mathrm{H}), 5.59(\mathrm{dd}, J=17.2,1.4 \mathrm{~Hz}, 1 \mathrm{H}), 5.32(\mathrm{dd}, J=11.0,1.4 \mathrm{~Hz}, 1 \mathrm{H}), 3.36(\mathrm{~s}, 2 \mathrm{H})$, 3.29 (s, 2H), 1.97-1.79 (m, 4H), 1.08-0.95 (m, 1H), 0.91-0.78 (m, 1H); ${ }^{13} \mathrm{C}$ NMR $(101$ MHz, Chloroform-d) $\delta$ 212.7, 137.2, 136.5, 134.7, 133.7, 131.0, 130.2, 128.4, 127.7, 127.5, 126.9, 126.3, 116.1, 70.1, 44.5, 41.5, 41.3, 14.8; HRMS: m/z (ESI) calculated $[\mathrm{M}+\mathrm{H}]^{+}: 319.1698$, found:319.1693. 
<smiles>C=Cc1ccccc1CC1(Cc2cccc(C(F)(F)F)c2)C(=O)CCCC1=O</smiles>

The title compound $\mathbf{3 b}$ was synthesized according to General Procedure (SI 2.3), and it was purified by column chromatography on silica gel.

${ }^{1} \mathrm{H}$ NMR (400 MHz, Chloroform- $d$ ) $\delta$ 7.56-7.40 (m, 2H), 7.37-7.09 (m, 5H), 7.05-6.89 $(\mathrm{m}, 2 \mathrm{H}), 5.61(\mathrm{~d}, J=17.2 \mathrm{~Hz}, 1 \mathrm{H}), 5.33(\mathrm{~d}, J=10.8 \mathrm{~Hz}, 1 \mathrm{H}), 3.36(\mathrm{~s}, 4 \mathrm{H}), 2.05-1.81$ (m, 4H), 1.22-0.98 (m, 1H), 0.94-0.72 (m, 1H); ${ }^{13} \mathrm{C}$ NMR (101 MHz, Chloroform- $d$ ) $\delta$ 212.2, 137.7, 137.2, 134.4, 133.8, 133.2, 131.1, 130.7 (q, $J=32.2 \mathrm{~Hz}), 128.8,127.9$, 127.7, 126.9 (q, $J=3.6 \mathrm{~Hz}), 126.4,123.9$ (q, $J=272.5 \mathrm{~Hz}), 123.6$ (q, $J=3.4 \mathrm{~Hz})$, 116.4, 70.0, 43.2, 42.0, 41.3, 14.8; HRMS: m/z (ESI) calculated $[\mathrm{M}+\mathrm{Na}]^{+}: 409.1391$, found:409.1383.<smiles>C=Cc1ccccc1CC1(Cc2ccc(OC)cc2)C(=O)CCCC1=O</smiles>

The title compound 3c was synthesized according to General Procedure (SI 2.3), and it was purified by column chromatography on silica gel.

${ }^{1} \mathrm{H}$ NMR (400 MHz, Chloroform- $d$ ) $\delta 7.47(\mathrm{dd}, J=7.6,1.7 \mathrm{~Hz}, 1 \mathrm{H}), 7.21-7.11(\mathrm{~m}, 2 \mathrm{H})$, 7.05-6.96 (m, 2H), $6.93(\mathrm{~d}, J=8.7 \mathrm{~Hz}, 2 \mathrm{H}), 6.73(\mathrm{~d}, J=8.7 \mathrm{~Hz}, 2 \mathrm{H}), 5.59$ (dd, $J=17.2$, $1.3 \mathrm{~Hz}, 1 \mathrm{H}), 5.32$ (dd, $J=11.0,1.3 \mathrm{~Hz}, 1 \mathrm{H}), 3.74(\mathrm{~s}, 3 \mathrm{H}), 3.34(\mathrm{~s}, 2 \mathrm{H}), 3.24(\mathrm{~s}, 2 \mathrm{H})$, 1.99-1.80 (m, 4H), 1.06-0.99 (m, 1H), 0.96-0.88 (m, 1H); ${ }^{13} \mathrm{C}$ NMR (101 MHz, Chloroform-d) $\delta 212.9,158.4,137.1,134.7,133.8,131.2,131.0,128.5,127.7,127.4$, 126.3, 116.1, 113.7, 70.2, 55.1, 43.8, 41.5, 41.1, 14.9; HRMS: m/z (ESI) calculated $\left[\mathrm{M}+\mathrm{NH}_{4}\right]^{+}: 366.2069$, found:366.2073.<smiles>C=Cc1ccccc1CC1(Cc2ccc(Br)cc2)C(=O)CCCC1=O</smiles>

The title compound 3d was synthesized according to General Procedure (SI 2.3), and it was purified by column chromatography on silica gel.

${ }^{1} \mathrm{H}$ NMR (400 MHz, Chloroform- $d$ ) $\delta 7.48$ (dd, $\left.J=7.7,1.6 \mathrm{~Hz}, 1 \mathrm{H}\right), 7.32$ (d, $J=8.4$ $\mathrm{Hz}, 2 \mathrm{H}), 7.23-7.12(\mathrm{~m}, 2 \mathrm{H}), 7.04-6.84(\mathrm{~m}, 4 \mathrm{H}), 5.60(\mathrm{dd}, J=17.2,1.4 \mathrm{~Hz}, 1 \mathrm{H}), 5.32$ $(\mathrm{dd}, J=11.0,1.4 \mathrm{~Hz}, 1 \mathrm{H}), 3.33(\mathrm{~s}, 2 \mathrm{H}), 3.25$ (s, 2H), 2.20-1.80 (m, 4H), 1.13-1.00 (m, 
1H), 0.97-0.83 (m, 1H); ${ }^{13} \mathrm{C}$ NMR (101 MHz, Chloroform-d) $\delta$ 212.4, 137.2, 135.6, 134.4, 133.3, 132.0, 131.5, 131.1, 127.8, 127.6, 126.4, 121.0, 116.3, 70.0, 43.2, 41.8, 41.5, 14.9; HRMS: m/z (ESI) calculated $[\mathrm{M}+\mathrm{K}]^{+}: 435.0362$, found:435.0365.<smiles>C=Cc1ccccc1CC1(Cc2cccc(Cl)c2)C(=O)CCCC1=O</smiles>

The title compound 3e was synthesized according to General Procedure (SI 2.3), and it was purified by column chromatography on silica gel.

${ }^{1} \mathrm{H}$ NMR (400 MHz, Chloroform- $d$ ) $\delta 7.48(\mathrm{dd}, J=7.6,1.6 \mathrm{~Hz}, 1 \mathrm{H}), 7.24-7.10(\mathrm{~m}, 4 \mathrm{H})$, 7.05-6.84 (m, 4H), $5.60(\mathrm{dd}, J=17.2,1.4 \mathrm{~Hz}, 1 \mathrm{H}), 5.32(\mathrm{dd}, J=11.0,1.4 \mathrm{~Hz}, 1 \mathrm{H}), 3.34$ $(\mathrm{s}, 2 \mathrm{H}), 3.26(\mathrm{~s}, 2 \mathrm{H}), 2.06-1.82(\mathrm{~m}, 4 \mathrm{H}), 1.15-1.02(\mathrm{~m}, 1 \mathrm{H}), 0.99-0.85(\mathrm{~m}, 1 \mathrm{H}) ;{ }^{13} \mathrm{C}$ NMR (101 MHz, Chloroform-d) $\delta$ 212.2, 138.7, 137.2, 134.5, 134.2, 133.3, 131.1, 130.3, 129.6, 128.5, 127.8, 127.7, 127.1, 126.4, 116.3, 69.9, 43.4, 41.8, 41.4, 14.9; HRMS: m/z (ESI) calculated $[\mathrm{M}+\mathrm{Na}]^{+}: 375.1128$, found:375.1125.<smiles>C=Cc1ccccc1CC1(Cc2ccc3c(c2)OCCO3)C(=O)CCCC1=O</smiles>

$3 f$

The title compound $3 f$ was synthesized according to General Procedure (SI 2.3), and it was purified by column chromatography on silica gel.

${ }^{1} \mathrm{H}$ NMR (400 MHz, Chloroform- $d$ ) $\delta 7.47$ (d, $\left.J=7.3 \mathrm{~Hz}, 1 \mathrm{H}\right), 7.22-7.12(\mathrm{~m}, 2 \mathrm{H}), 7.05-$ $6.95(\mathrm{~m}, 2 \mathrm{H}), 6.68(\mathrm{~d}, J=8.2 \mathrm{~Hz}, 1 \mathrm{H}), 6.54(\mathrm{~s}, 1 \mathrm{H}), 6.48(\mathrm{~d}, J=8.2 \mathrm{~Hz}, 1 \mathrm{H}), 5.59$ (d, $J$ $=17.2 \mathrm{~Hz}, 1 \mathrm{H}), 5.31(\mathrm{~d}, J=11.0 \mathrm{~Hz}, 1 \mathrm{H}), 4.19(\mathrm{~s}, 4 \mathrm{H}), 3.32(\mathrm{~s}, 2 \mathrm{H}), 3.18(\mathrm{~s}, 2 \mathrm{H}), 2.06-$ $1.83(\mathrm{~m}, 4 \mathrm{H}), 1.12-0.94(\mathrm{~m}, 2 \mathrm{H}) ;{ }^{13} \mathrm{C}$ NMR (101 MHz, Chloroform-d) $\delta$ 212.7, 143.2, 142.4, 137.2, 134.7, 133.8, 131.0, 129.7, 127.7, 127.4, 126.3, 123.2, 118.9, 117.0, 116.1, 70.0, 64.3, 64.2, 43.9, 41.5, 41.2, 14.9; HRMS: m/z (ESI) calculated $[\mathrm{M}+\mathrm{H}]^{+}: 377.1753$, found:377.1754.<smiles>C=Cc1ccccc1CC1(Cc2ccco2)C(=O)CCCC1=O</smiles>

The title compound 3g was synthesized according to General Procedure (SI 2.3), and it was purified by column chromatography on silica gel.

${ }^{1} \mathrm{H}$ NMR (400 MHz, Chloroform- $d$ ) $\delta 7.48$ (d, $\left.J=7.4 \mathrm{~Hz}, 1 \mathrm{H}\right)$, 7.24-7.12 (m, 3H), 7.04 $-6.86(\mathrm{~m}, 2 \mathrm{H}), 6.20(\mathrm{~s}, 1 \mathrm{H}), 5.94(\mathrm{~d}, J=2.7 \mathrm{~Hz}, 1 \mathrm{H}), 5.60(\mathrm{~d}, J=17.2 \mathrm{~Hz}, 1 \mathrm{H}), 5.31(\mathrm{~d}$, $J=11.0 \mathrm{~Hz}, 1 \mathrm{H}), 3.32(\mathrm{~s}, 2 \mathrm{H}), 3.27$ (s, 2H), 2.29-2.20 (m, 2H), 2.01-1.89 (m, 2H), 1.30$1.15(\mathrm{~m}, 2 \mathrm{H}) ;{ }^{13} \mathrm{C}$ NMR (101 MHz, Chloroform-d) $\delta 211.7,151.1,141.3,137.2,134.4$, 
133.3, 131.1, 127.8, 127.6, 126.3, 116.4, 110.5, 107.9, 67.7, 41.2, 40.7, 36.3, 15.5; HRMS: $\mathrm{m} / \mathrm{z}(\mathrm{ESI})$ calculated $[\mathrm{M}+\mathrm{Na}]^{+}: 331.1310$, found:331.1308.<smiles>C=Cc1ccccc1CC1(Cc2cccs2)C(=O)CCCC1=O</smiles>

The title compound $\mathbf{3 h}$ was synthesized according to General Procedure (SI 2.3), and it was purified by column chromatography on silica gel.

${ }^{1} \mathrm{H}$ NMR (400 MHz, Chloroform- $d$ ) $\delta 7.48$ (dd, $\left.J=7.6,1.6 \mathrm{~Hz}, 1 \mathrm{H}\right), 7.24-7.13(\mathrm{~m}, 2 \mathrm{H})$, $7.06(\mathrm{dd}, J=5.2,1.2 \mathrm{~Hz}, 1 \mathrm{H}), 7.00(\mathrm{dd}, J=7.6,1.6 \mathrm{~Hz}, 1 \mathrm{H}), 6.93(\mathrm{dd}, J=17.2,11.0$ $\mathrm{Hz}, 1 \mathrm{H}), 6.83$ (dd, $J=5.2,3.2 \mathrm{~Hz}, 1 \mathrm{H}), 6.68(\mathrm{~d}, J=3.2 \mathrm{~Hz}, 1 \mathrm{H}), 5.61$ (dd, $J=17.2,1.4$ $\mathrm{Hz}, 1 \mathrm{H}), 5.32(\mathrm{dd}, J=11.0,1.4 \mathrm{~Hz}, 1 \mathrm{H}), 3.51(\mathrm{~s}, 2 \mathrm{H}), 3.29(\mathrm{~s}, 2 \mathrm{H}), 2.17-2.07(\mathrm{~m}, 2 \mathrm{H})$, 1.96-1.84 (m, 2H), 1.23-0.94 (m, 2H); ${ }^{13} \mathrm{C}$ NMR (101 MHz, Chloroform-d) $\delta$ 212.4, 138.2, 137.2, 134.3, 133.2, 131.1, 127.8, 127.7, 126.7, 126.3, 124.5, 116.4, 70.2, 41.7, 41.4, 37.8, 15.1; HRMS: m/z (ESI) calculated $[\mathrm{M}+\mathrm{Na}]^{+}: 347.1082$, found:347.1081.<smiles>C=CCC1(Cc2ccccc2C=C)C(=O)CCCC1=O</smiles>

$3 \mathbf{i}$

The title compound 3i was synthesized according to General Procedure (SI 2.4), and it was purified by column chromatography on silica gel.

${ }^{1} \mathrm{H}$ NMR (400 MHz, Chloroform- $d$ ) $\delta 7.48(\mathrm{dd}, J=7.8,1.6 \mathrm{~Hz}, 1 \mathrm{H}), 7.18(\mathrm{~m}, 2 \mathrm{H}), 7.00$ $6.86(\mathrm{~m}, 2 \mathrm{H}), 5.60(\mathrm{dd}, J=17.2,1.4 \mathrm{~Hz}, 1 \mathrm{H}), 5.49$ (ddt, $J=17.2,10.0,7.4 \mathrm{~Hz}, 1 \mathrm{H})$, $5.30(\mathrm{dd}, J=11.0,1.4 \mathrm{~Hz}, 1 \mathrm{H}), 5.13-4.91(\mathrm{~m}, 2 \mathrm{H}), 3.20(\mathrm{~s}, 2 \mathrm{H}), 2.70(\mathrm{~d}, J=7.4 \mathrm{~Hz}$, 2H), 2.40-2.29 (m, 2H), 2.10-2.01 (m, 2H), 1.67-1.59 (m, 1H), 1.41-1.29 (m, 1H); ${ }^{13} \mathrm{C}$ NMR (101 MHz, Chloroform- $d$ ) $\delta$ 211.8, 137.3, 134.4, 133.7, 132.5, 131.0, 127.8, 127.6, 126.3, 119.6, 116.4, 68.8, 42.2, 41.2, 41.1, 15.5; HRMS: m/z (ESI) calculated $[\mathrm{M}+\mathrm{Na}]^{+}: 291.1361$, found: 291.1355 .<smiles>C=Cc1ccccc1CC1(CCC(=O)OC(C)(C)C)C(=O)CCCC1=O</smiles>

3j

The title compound $\mathbf{3 j}$ was synthesized according to General Procedure (SI 2.5), and it was purified by column chromatography on silica gel.

${ }^{1} \mathrm{H}$ NMR (400 MHz, Chloroform- $d$ ) $\delta 7.49$ (d, $\left.J=7.8 \mathrm{~Hz}, 1 \mathrm{H}\right), 7.21$ (dd, $J=7.8,7.2$ $\mathrm{Hz}, 1 \mathrm{H}), 7.15(\mathrm{dd}, J=7.6,7.2 \mathrm{~Hz}, 1 \mathrm{H}), 7.00-6.80(\mathrm{~m}, 2 \mathrm{H}), 5.61(\mathrm{~d}, J=17.2 \mathrm{~Hz}, 1 \mathrm{H})$, $5.31(\mathrm{~d}, J=11.0 \mathrm{~Hz}, 1 \mathrm{H}), 3.17(\mathrm{~s}, 2 \mathrm{H}), 2.52-2.41(\mathrm{~m}, 2 \mathrm{H}), 2.29-2.21(\mathrm{~m}, 2 \mathrm{H}), 2.15-2.05$ $(\mathrm{m}, 2 \mathrm{H}), 2.04-1.92(\mathrm{~m}, 2 \mathrm{H}), 1.76-1.65(\mathrm{~m}, 1 \mathrm{H}), 1.50-1.38(\mathrm{~m}, 10 \mathrm{H}) ;{ }^{13} \mathrm{C}$ NMR $(101$ 
MHz, Chloroform-d) $\delta$ 211.6, 171.6, 137.4, 134.3, 133.4, 130.9, 127.8, 127.7, 126.3, 116.6, 80.6, 67.9, 42.0, 40.7, 31.9, 31.3, 28.0, 16.0; HRMS: m/z (ESI) calculated $\left[\mathrm{M}+\mathrm{NH}_{4}\right]^{+}: 374.2331$, found:374.2325.<smiles>C=Cc1cc(Cl)ccc1CC1(C)C(=O)CC(C)(C)CC1=O</smiles>

The title compound 3k was synthesized according to General Procedure (SI 2.6), and it was purified by column chromatography on silica gel.

${ }^{1} \mathrm{H}$ NMR (400 MHz, Chloroform- $d$ ) $\delta 7.46(\mathrm{~d}, J=2.4 \mathrm{~Hz}, 1 \mathrm{H}), 7.14$ (dd, $J=8.2,2.4$ $\mathrm{Hz}, 1 \mathrm{H}), 6.91$ (d, $J=8.2 \mathrm{~Hz}, 1 \mathrm{H}), 6.85(\mathrm{dd}, J=17.2,11.0 \mathrm{~Hz}, 1 \mathrm{H}), 5.63$ (dd, $J=17.2$, $1.1 \mathrm{~Hz}, 1 \mathrm{H}), 5.36(\mathrm{dd}, J=11.0,1.1 \mathrm{~Hz}, 1 \mathrm{H}), 3.11(\mathrm{~s}, 2 \mathrm{H}), 2.48(\mathrm{~d}, J=15.0 \mathrm{~Hz}, 1 \mathrm{H})$, $2.30(\mathrm{~d}, J=15.0 \mathrm{~Hz}, 2 \mathrm{H}), 1.23(\mathrm{~s}, 3 \mathrm{H}), 0.95(\mathrm{~s}, 3 \mathrm{H}), 0.86(\mathrm{~s}, 3 \mathrm{H}) ;{ }^{13} \mathrm{C} \mathrm{NMR}(101 \mathrm{MHz}$, Chloroform- $d$ ) $\delta 210.8,139.5,133.5,133.4,132.3,132.0,127.6,126.2,117.9,64.1$, 53.3, 40.0, 30.2, 29.8, 27.8, 20.0; HRMS: $\mathrm{m} / \mathrm{z}(\mathrm{ESI})$ calculated $[\mathrm{M}+\mathrm{Na}]^{+}: 327.1128$, found:327.1131.<smiles>O=C1CCC[C@@]2(O)[C@H](Cc3ccccc3)c3ccccc3C[C@]12O</smiles>

The title compound 2a was synthesized according to General Procedure (SI 4.1), and it was purified by column chromatography on silica gel (86\% yield, 99\% ee, $63.6 \mathrm{mg}$, white solid).

${ }^{1} \mathrm{H}$ NMR (400 MHz, Chloroform- $d$ ) $\delta 7.32$ (d, $\left.J=7.8 \mathrm{~Hz}, 1 \mathrm{H}\right), 7.22-7.10$ (m, 2H), 7.05 (d, $J=7.4 \mathrm{~Hz}, 1 \mathrm{H}), 3.44$ (d, $J=17.0 \mathrm{~Hz}, 1 \mathrm{H}), 3.15$ (dd, $J=9.6,6.0 \mathrm{~Hz}, 1 \mathrm{H}), 2.81-2.67$ $(\mathrm{m}, 1 \mathrm{H}), 2.61(\mathrm{~d}, J=17.0 \mathrm{~Hz}, 1 \mathrm{H}), 2.47-2.28(\mathrm{~m}, 2 \mathrm{H}), 2.20-2.04(\mathrm{~m}, 1 \mathrm{H}), 1.95-1.85(\mathrm{~m}$, $1 \mathrm{H}), 1.84-1.75(\mathrm{~m}, 1 \mathrm{H}), 1.73-1.62(\mathrm{~m}, 1 \mathrm{H}), 1.48(\mathrm{dd}, J=15.6,6.0 \mathrm{~Hz}, 1 \mathrm{H}), 1.29(\mathrm{~s}$, $12 \mathrm{H}), 1.15$ (s, 3H), 1.19-1.05 (m, 1H); ${ }^{13} \mathrm{C}$ NMR (101 MHz, Chloroform-d) $\delta 214.5$, 139.2, 132.2, 128.8, 127.0, 126.3, 126.1, 83.6, 78.7, 53.7, 42.8, 39.9, 36.0 25.7, 24.9, 24.7, 19.8, 14.3; HRMS: $\mathrm{m} / \mathrm{z}$ (ESI) calculated $[\mathrm{M}+\mathrm{Na}]^{+}: 393.2213$, found:393.2214. $[\alpha]_{\mathrm{D}}^{25}=+65.2\left(c=0.17, \mathrm{CHCl}_{3}\right)$.

The enantiomeric excess of $\mathbf{2 a}$ was determined by chiral HPLC analysis compared to the corresponding racemate.

Conditions: ChiralPark IA column; hexane $/{ }^{i} \mathrm{PrOH}=98: 2$; flow rate $=0.5 \mathrm{~mL} / \mathrm{min} ; \lambda=$ $272 \mathrm{~nm} ; \mathrm{t}_{\mathrm{R} 1}$ (major) $=39.7 \mathrm{~min} ; \mathrm{t}_{\mathrm{R} 2}($ minor $)=36.8 \mathrm{~min}$. 

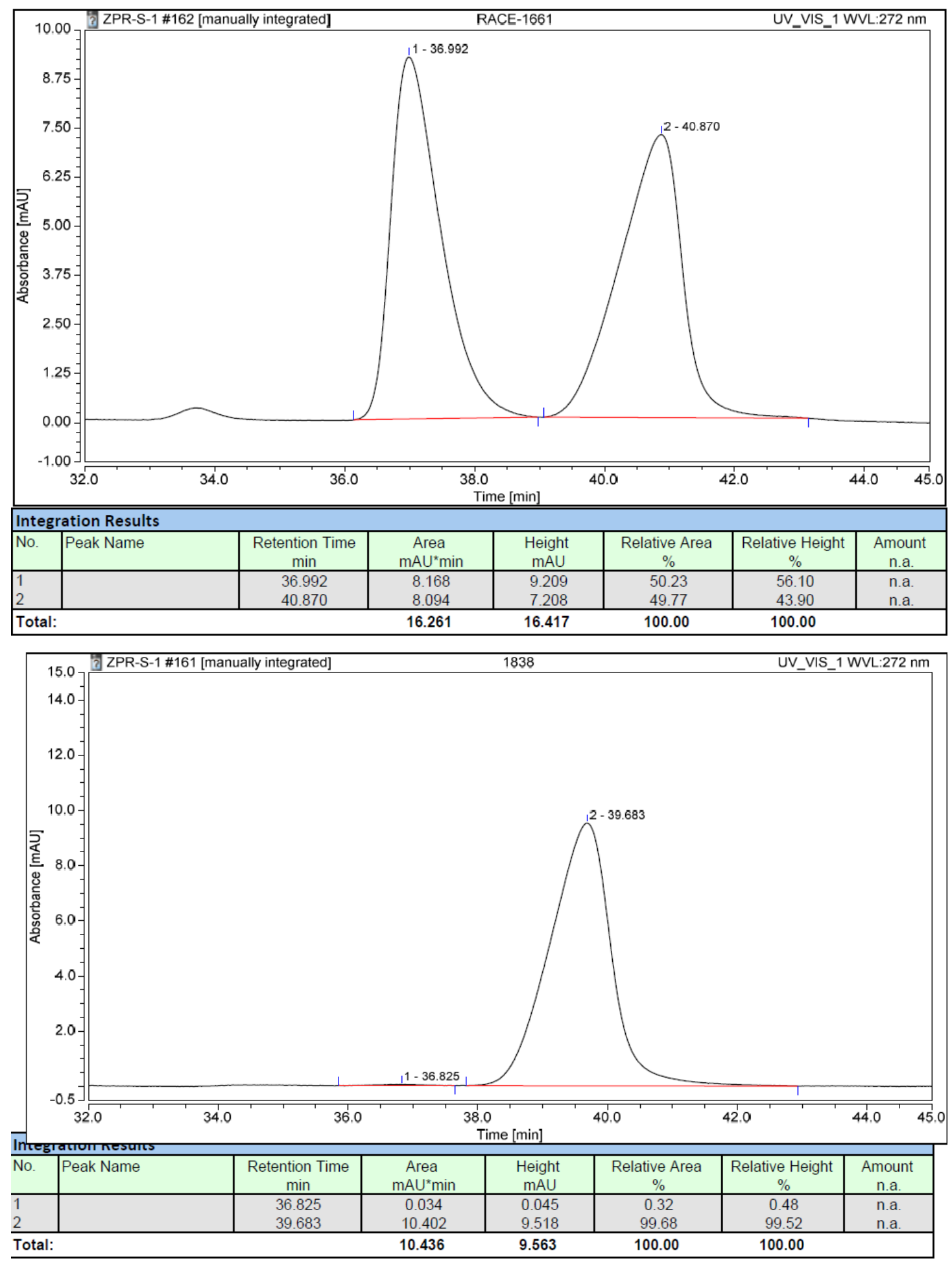<smiles>O=C1CCCC23OB(O)CC2c2ccccc2CC13Br</smiles>

The title compound 2a' was synthesized according to General Procedure (SI 3), and it was purified by column chromatography on silica gel(white solid). 
${ }^{1} \mathrm{H}$ NMR (600 MHz, Chloroform- $d$ ) $\delta$ 7.21-7.15(m, 2H), $7.12(\mathrm{dd}, J=7.4,7.2 \mathrm{~Hz}, 1 \mathrm{H})$, 7.07 (d, $J=7.4 \mathrm{~Hz}, 1 \mathrm{H}), 4.48(\mathrm{br}, 1 \mathrm{H}), 3.37$ (t, $J=9.6 \mathrm{~Hz}, 1 \mathrm{H}), 3.25(\mathrm{~d}, J=15.7 \mathrm{~Hz}$, $1 \mathrm{H}), 2.59-2.50(\mathrm{~m}, 2 \mathrm{H}), 2.48-2.41(\mathrm{~m}, 1 \mathrm{H}), 2.19-2.02(\mathrm{~m}, 2 \mathrm{H}), 1.96-1.91(\mathrm{~m}, 1 \mathrm{H}), 1.87-$ $1.79(\mathrm{~m}, 2 \mathrm{H}), 1.10(\mathrm{~s}, 1 \mathrm{H}), 1.15-1.07(\mathrm{~m}, 1 \mathrm{H}) ;{ }^{13} \mathrm{C}$ NMR $(151 \mathrm{MHz}$, Chloroform- $d) \delta$ 213.2, 139.8, 132.6, 128.7, 128.5, 126.7, 125.9, 89.1, 53.8, 46.5, 37.8, 36.8, 35.3, 20.6, 17.8; HRMS: $\mathrm{m} / \mathrm{z}$ (ESI) calculated $[\mathrm{M}+\mathrm{Na}]^{+}: 293.1325$, found:293.1325.<smiles>Cc1ccc2c(c1)[C@H](C[Pb])[C@@]1(C)CCCC(=O)[C@@]1(C)C2</smiles>

The title compound $\mathbf{2 b}$ was synthesized according to General Procedure (SI 4.1), and it was purified by column chromatography on silica gel (96\% yield, $98 \%$ ee, $73.8 \mathrm{mg}$, white solid).

${ }^{1} \mathrm{H}$ NMR (600 MHz, Chloroform- $d$ ) $\delta 7.14(\mathrm{~s}, 1 \mathrm{H}), 6.97(\mathrm{~d}, J=7.8 \mathrm{~Hz}, 1 \mathrm{H}), 6.93(\mathrm{~d}, J$ $=7.8 \mathrm{~Hz}, 1 \mathrm{H}), 3.39(\mathrm{~d}, J=16.8 \mathrm{~Hz}, 1 \mathrm{H}), 3.17-3.07(\mathrm{~m}, 1 \mathrm{H}), 2.78-2.70(\mathrm{~m}, 1 \mathrm{H}), 2.56$ $(\mathrm{d}, J=16.8 \mathrm{~Hz}, 1 \mathrm{H}), 2.31(\mathrm{~s}, 3 \mathrm{H}), 2.38-2.24(\mathrm{~m}, 2 \mathrm{H}), 2.16-2.06(\mathrm{~m}, 1 \mathrm{H}), 1.94-1.84(\mathrm{~m}$, $1 \mathrm{H}), 1.84-1.75(\mathrm{~m}, 1 \mathrm{H}), 1.72-1.61(\mathrm{~m}, 1 \mathrm{H}), 1.45(\mathrm{dd}, J=15.4,6.1 \mathrm{~Hz}, 2 \mathrm{H}), 1.31-1.26$ $(\mathrm{m}, 12 \mathrm{H}), 1.13(\mathrm{~s}, 3 \mathrm{H}), 1.20-1.01(\mathrm{~m}, 1 \mathrm{H}) ;{ }^{13} \mathrm{C}$ NMR $(151 \mathrm{MHz}$, Chloroform- $d$ ) $\delta 214.6$, 139.0, 135.7, 129.1, 128.8, 127.7, 127.0, 83.6, 78.8, 53.8, 42.8, 39.5, 36.0, 25.7, 24.9, 24.7, 21.2, 19.9, 14.3; HRMS: $\mathrm{m} / \mathrm{z}$ (ESI) calculated $\left[\mathrm{M}+\mathrm{NH}_{4}\right]^{+}: 402.2816$, found:402.2813. $[\alpha]_{\mathrm{D}}{ }^{25}=+47.1\left(c=0.10, \mathrm{CHCl}_{3}\right)$.

The enantiomeric excess of $\mathbf{2} \mathbf{b}$ was determined by chiral HPLC analysis compared to the corresponding racemate.

Conditions: ChiralPark IC column; hexane $/{ }^{i} \mathrm{PrOH}=90: 10$; flow rate $=1.0 \mathrm{~mL} / \mathrm{min} ; \lambda=$ $272 \mathrm{~nm} ; \mathrm{t}_{\mathrm{R} 1}($ major $)=11.5 \mathrm{~min} ; \mathrm{t}_{\mathrm{R} 2}($ minor $)=13.4 \mathrm{~min}$. 


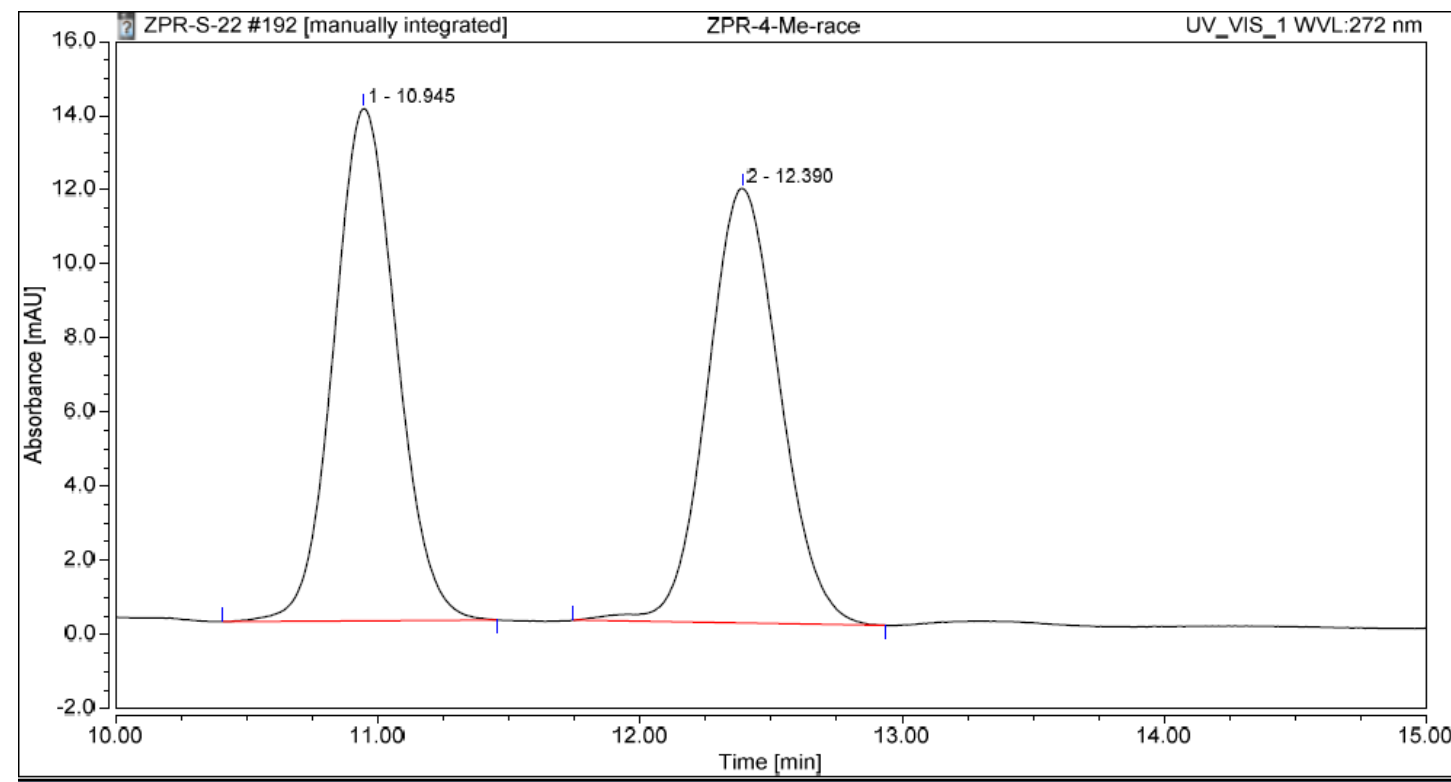

\begin{tabular}{|c|c|c|c|c|c|c|c|}
\hline \multicolumn{8}{|c|}{ Integration Results } \\
\hline No. & Peak Name & $\begin{array}{c}\text { Retention Time } \\
\text { min }\end{array}$ & $\begin{array}{c}\text { Area } \\
\mathrm{mAU}^{*} \min \end{array}$ & $\begin{array}{c}\text { Height } \\
\text { mAU }\end{array}$ & $\begin{array}{c}\text { Relative Area } \\
\% \\
\end{array}$ & $\begin{array}{c}\text { Relative Height } \\
\% \\
\end{array}$ & $\begin{array}{c}\text { Amount } \\
\text { n.a. }\end{array}$ \\
\hline 1 & & 10.945 & 3.847 & 13.834 & 50.58 & 54.12 & n.a. \\
\hline$\underline{2}$ & & 12.390 & 3.758 & 11.730 & 49.42 & 45.88 & n.a. \\
\hline \multicolumn{3}{|c|}{ Total: } & 7.605 & 25.563 & 100.00 & 100.00 & \\
\hline
\end{tabular}

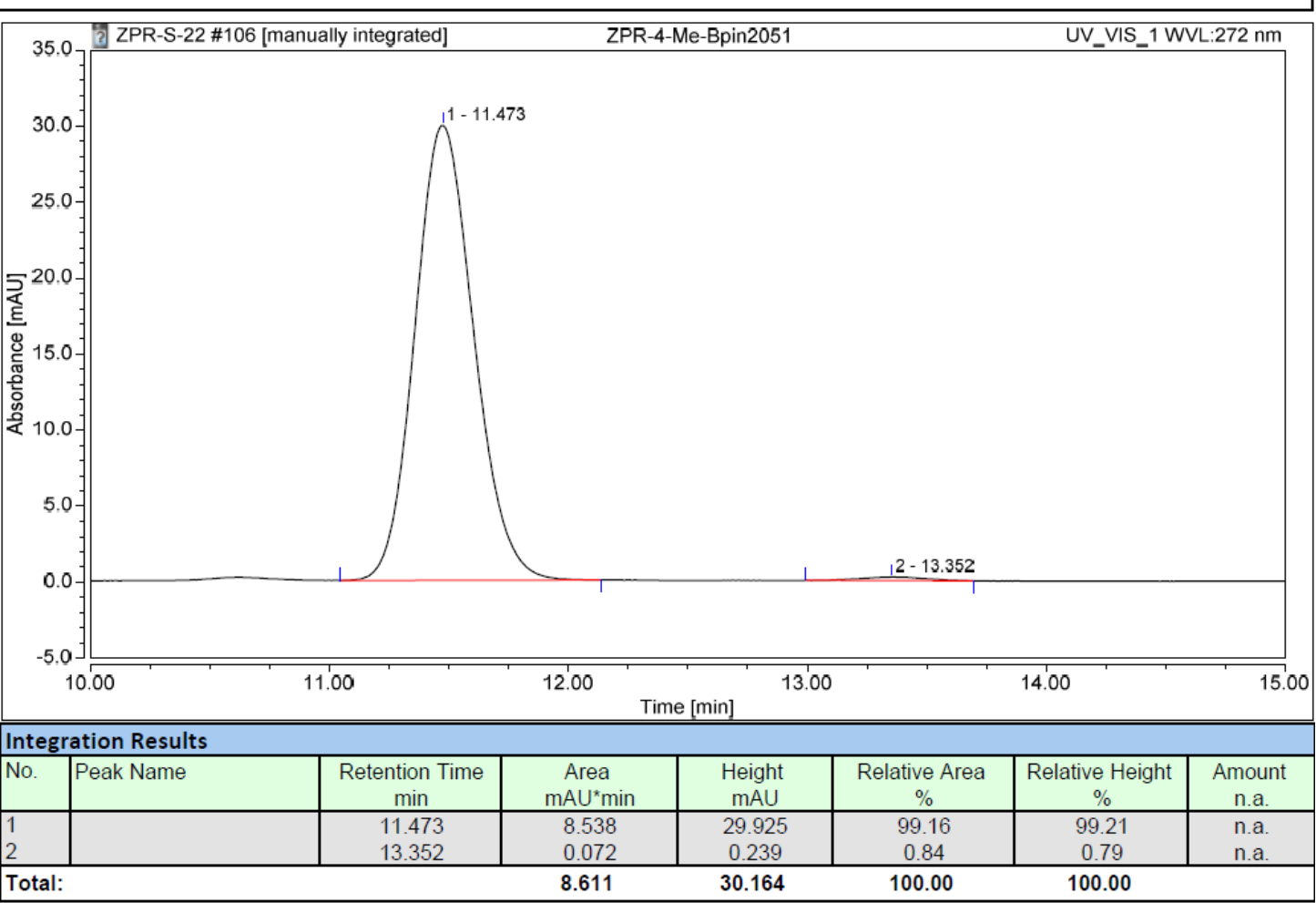<smiles>C[C@]12Cc3ccc(Cl)cc3[C@@H](C[Pb])[C@@]1(O)CCCC2=O</smiles> 
The title compound 2c was synthesized according to General Procedure (SI 4.1), and it was purified by column chromatography on silica gel (92\% yield, 99\% ee, $74.3 \mathrm{mg}$, white solid).

${ }^{1} \mathrm{H}$ NMR (400 MHz, Chloroform- $d$ ) $\delta 7.32(\mathrm{~s}, 1 \mathrm{H}), 7.12(\mathrm{~d}, J=8.0 \mathrm{~Hz}, 1 \mathrm{H}), 6.98(\mathrm{~d}, J$ $=8.0 \mathrm{~Hz}, 1 \mathrm{H}), 3.38(\mathrm{~d}, J=17.1 \mathrm{~Hz}, 1 \mathrm{H}), 3.17-3.06(\mathrm{~m}, 1 \mathrm{H}), 2.80-2.66(\mathrm{~m}, 1 \mathrm{H}), 2.57$ $(\mathrm{d}, J=17.1 \mathrm{~Hz}, 1 \mathrm{H}), 2.48-2.27(\mathrm{~m}, 2 \mathrm{H}), 2.20-2.03(\mathrm{~m}, 1 \mathrm{H}), 1.94-1.86(\mathrm{~m}, 1 \mathrm{H}), 1.84-$ $1.74(\mathrm{~m}, 1 \mathrm{H}), 1.70-1.63(\mathrm{~m}, 1 \mathrm{H}), 1.39$ (dd, $J=15.5,6.2 \mathrm{~Hz}, 1 \mathrm{H}), 1.29$ (s, 12H), 1.13 (s, $3 \mathrm{H}), 1.19-1.10(\mathrm{~m}, 1 \mathrm{H}) ;{ }^{13} \mathrm{C}$ NMR (101 MHz, Chloroform- $d$ ) $\delta$ 214.0, 141.2, 132.2, 130.7, 130.2, 127.2, 126.2, 83.7, 78.4, 53.5, 42.7, 39.2, 35.9, 25.7, 24.8, 24.7, 19.7, 14.4; HRMS: $\mathrm{m} / \mathrm{z}(\mathrm{ESI})$ calculated $\left[\mathrm{M}+\mathrm{NH}_{4}\right]^{+}: 422.2269$, found:422.2263. $[\alpha]_{\mathrm{D}}{ }^{25}=+43.9(c$ $\left.=0.13, \mathrm{CHCl}_{3}\right)$.

The enantiomeric excess of $\mathbf{2 c}$ was determined by chiral HPLC analysis compared to the corresponding racemate.

Conditions: ChiralPark IB column; hexane $/{ }^{i} \mathrm{PrOH}=90: 10$; flow rate $=0.8 \mathrm{~mL} / \mathrm{min} ; \lambda=$ $272 \mathrm{~nm} ; \mathrm{t}_{\mathrm{R} 1}$ (major) $=7.6 \mathrm{~min} ; \mathrm{t}_{\mathrm{R} 2}($ minor $)=7.1 \mathrm{~min}$.

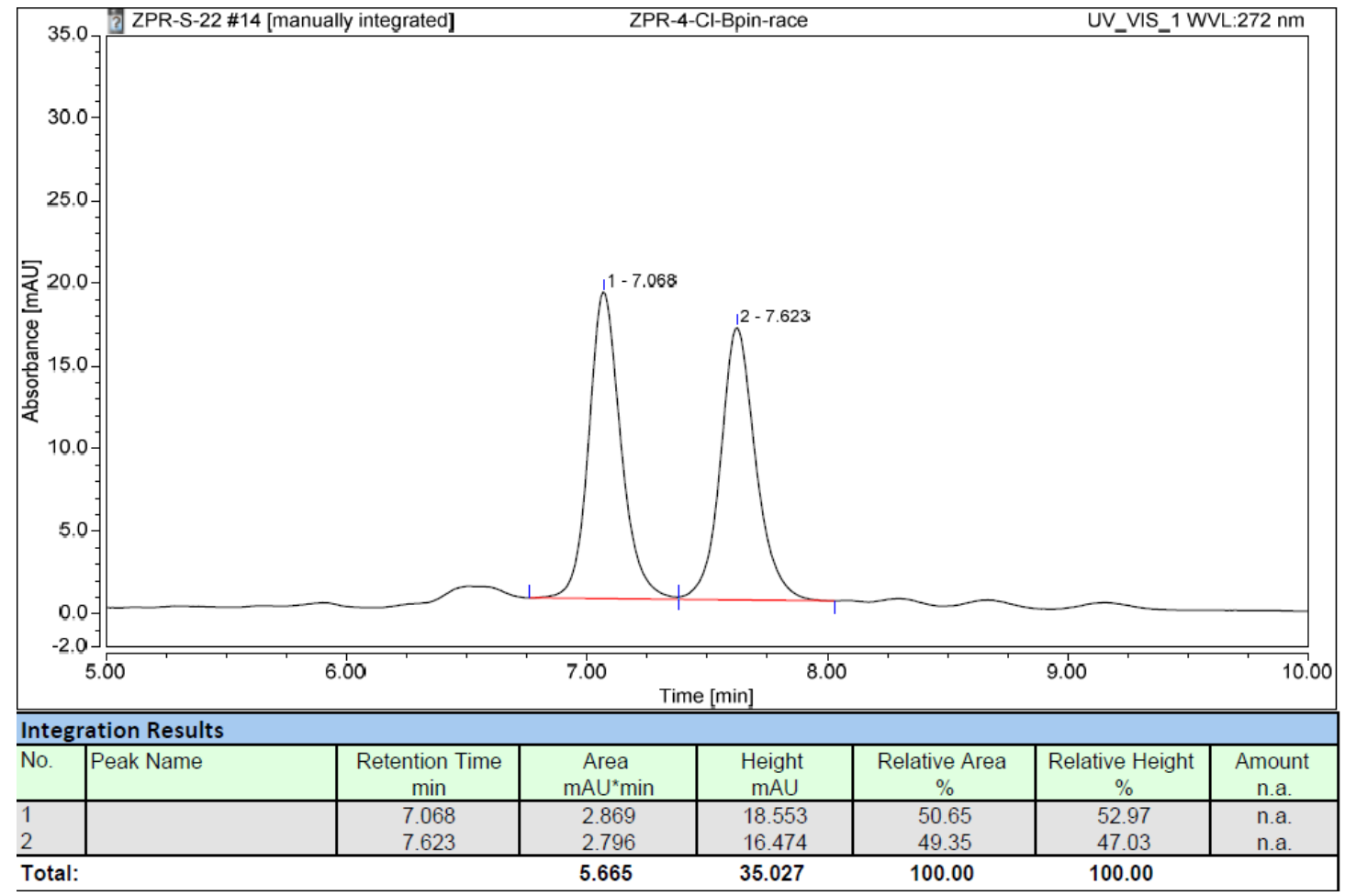




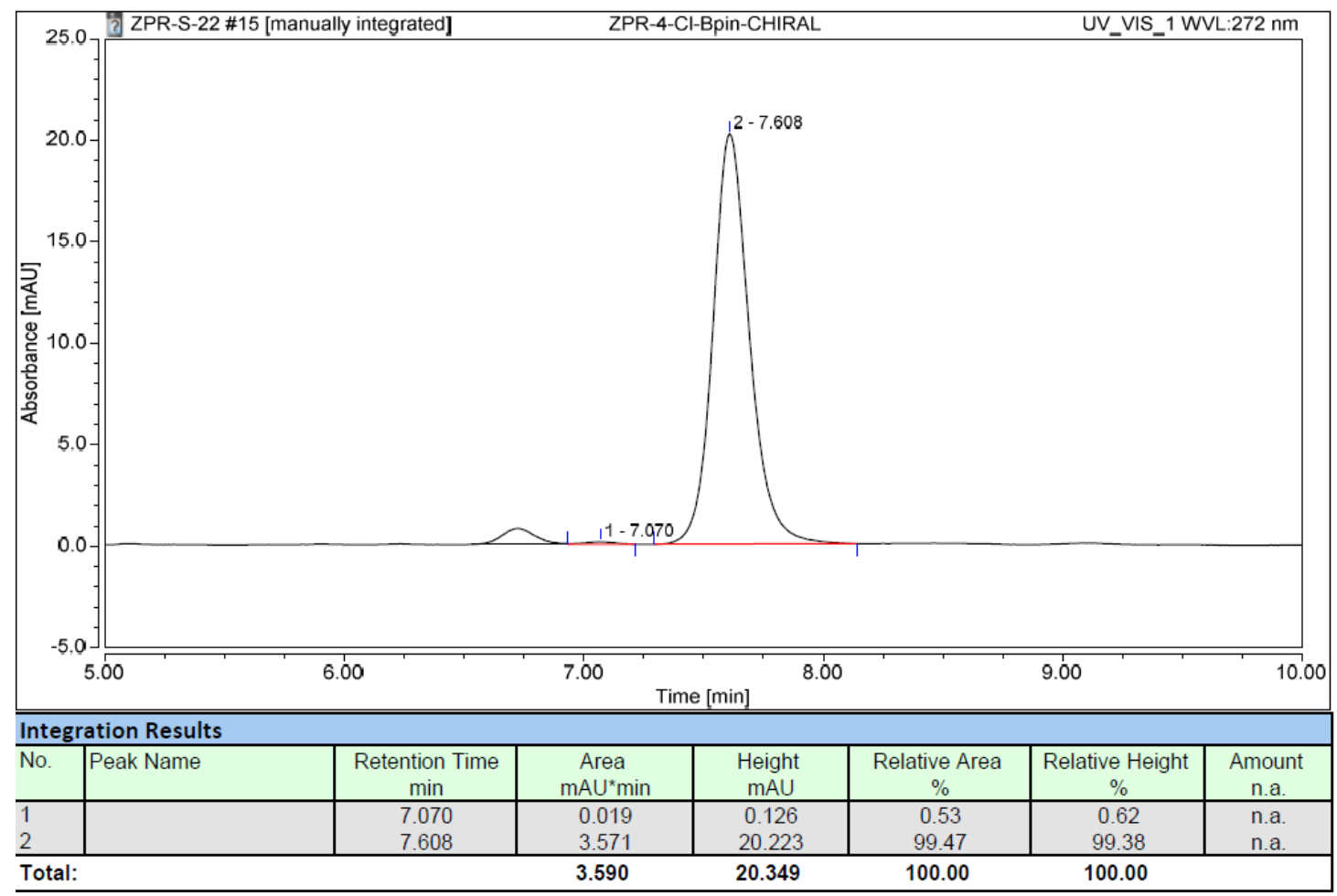<smiles>C[C@]12Cc3ccc(F)cc3[C@H](Cc3ccccc3)[C@@]1(O)CCCC2=O</smiles>

The title compound 2d was synthesized according to General Procedure (SI 4.1), and it was purified by column chromatography on silica gel ( $87 \%$ yield, $98 \% e e, 67.5 \mathrm{mg}$, white solid).

${ }^{1} \mathrm{H}$ NMR (400 MHz, Chloroform- $d$ ) $\delta$ 7.09-6.95 (m, 2H), 6.92-6.76 (m, 1H), 3.38 (d, $J$ $=16.8 \mathrm{~Hz}, 1 \mathrm{H}), 3.11(\mathrm{dd}, J=9.7,6.0 \mathrm{~Hz}, 1 \mathrm{H}), 2.80-2.66(\mathrm{~m}, 1 \mathrm{H}), 2.58(\mathrm{~d}, J=16.8 \mathrm{~Hz}$, $1 \mathrm{H}), 2.45-2.29(\mathrm{~m}, 2 \mathrm{H}), 2.19-2.06(\mathrm{~m}, 1 \mathrm{H}), 1.94-1.85(\mathrm{~m}, 1 \mathrm{H}), 1.84-1.76(\mathrm{~m}, 1 \mathrm{H}), 1.68-$ $1.61(\mathrm{~m}, 1 \mathrm{H}), 1.39(\mathrm{dd}, \mathrm{J}=15.4,6.0 \mathrm{~Hz}, 1 \mathrm{H}), 1.29(\mathrm{~s}, 12 \mathrm{H}), 1.13(\mathrm{~s}, 3 \mathrm{H}), 1.18-1.06(\mathrm{~m}$, $1 \mathrm{H}) ;{ }^{13} \mathrm{C}$ NMR (101 MHz, Chloroform- $d$ ) $\delta 214.2,161.7(\mathrm{~d}, J=243.4 \mathrm{~Hz}), 141.6(\mathrm{~d}, J$ $=6.8 \mathrm{~Hz}), 130.2(\mathrm{~d}, J=8.1 \mathrm{~Hz}), 127.7(\mathrm{~d}, J=3.0 \mathrm{~Hz}), 113.7(\mathrm{~d}, J=22.1 \mathrm{~Hz}), 113.3(\mathrm{~d}$, $J=21.4 \mathrm{~Hz}), 83.7,78.4,53.6,42.9,39.1,35.9,25.7,24.9,24.7,19.7,14.3 ; \mathrm{HRMS}: \mathrm{m} / \mathrm{z}$ $\left(\right.$ ESI) calculated $[\mathrm{M}+\mathrm{Na}]^{+}: 411.2119$, founded:411.2124. $[\alpha]_{\mathrm{D}}{ }^{25}=+80.8(c=0.10$, $\left.\mathrm{CHCl}_{3}\right)$.

The enantiomeric excess of $\mathbf{2 d}$ was determined by chiral HPLC analysis compared to the corresponding racemate.

Conditions: ChiralPark IB column; hexane $/{ }^{i} \mathrm{PrOH}=90: 10$; flow rate $=0.8 \mathrm{~mL} / \mathrm{min} ; \lambda=$ $272 \mathrm{~nm} ; \mathrm{t}_{\mathrm{R} 1}($ major $)=7.6 \mathrm{~min} ; \mathrm{t}_{\mathrm{R} 2}($ minor $)=7.1 \mathrm{~min}$. 

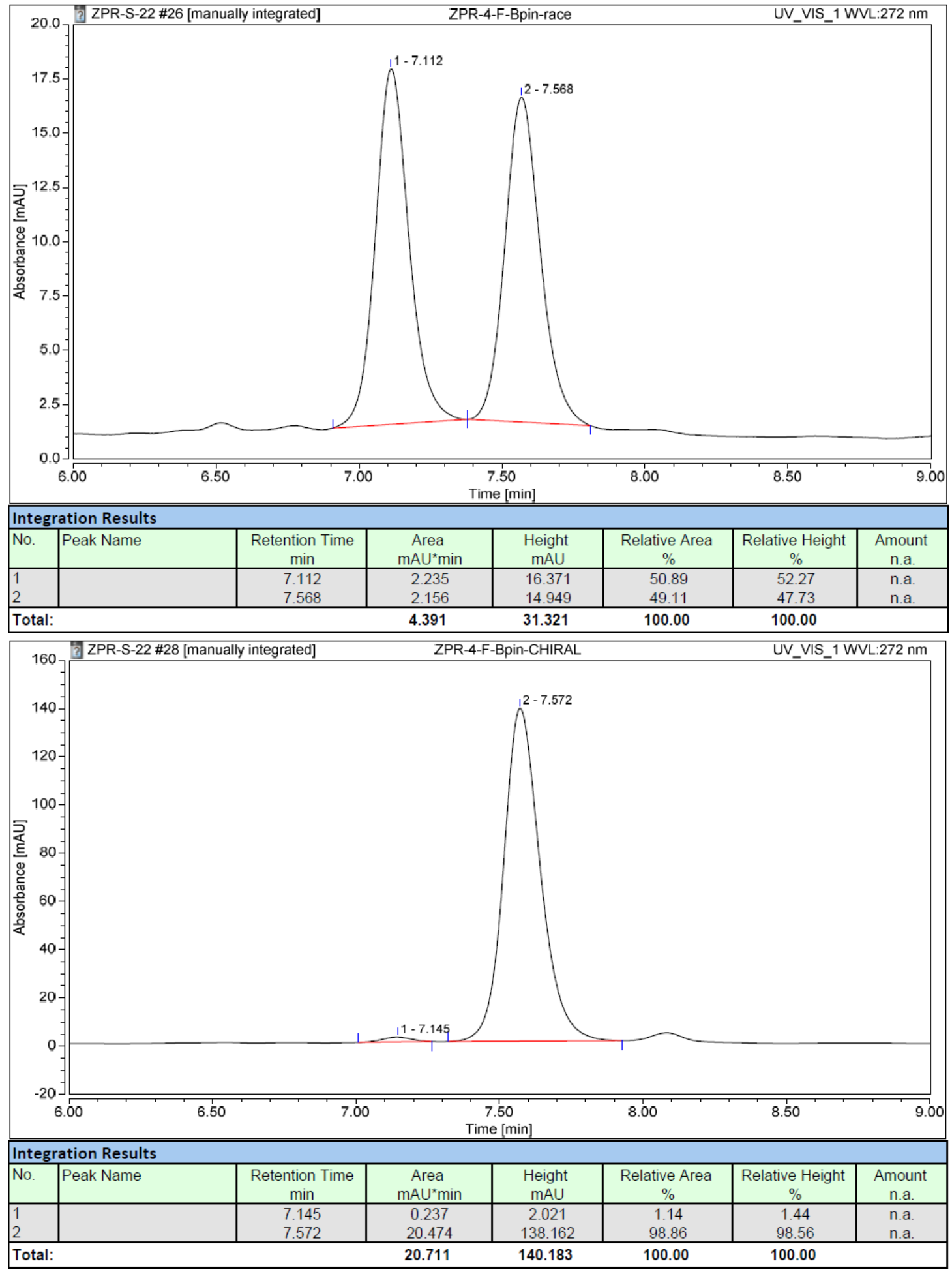<smiles>C[C@]12CCCC(=O)[C@@]1(O)Cc1cc(F)ccc1C2C[Pb]</smiles> 
The title compound 2e was synthesized according to General Procedure (SI 4.1), and it was purified by column chromatography on silica gel ( $84 \%$ yield, 99\% ee, $65.2 \mathrm{mg}$, white solid).

${ }^{1} \mathrm{H}$ NMR (600 MHz, Chloroform- $d$ ) $\delta 7.28(\mathrm{~m}, 1 \mathrm{H}), 6.88(\mathrm{~m}, 1 \mathrm{H}), 6.74(\mathrm{~m}, 1 \mathrm{H}), 3.41$ $(\mathrm{d}, J=17.1 \mathrm{~Hz}, 1 \mathrm{H}), 3.19-3.03(\mathrm{~m}, 1 \mathrm{H}), 2.77-2.67(\mathrm{~m}, 1 \mathrm{H}), 2.57(\mathrm{~d}, J=17.1 \mathrm{~Hz}, 1 \mathrm{H})$, 2.41-2.30 (m, 2H), 2.16-2.06 (m, 1H), 1.93-1.85 (m, 1H), 1.82-1.76 (m, 1H), 1.68-1.59 $(\mathrm{m}, 1 \mathrm{H}), 1.44(\mathrm{dd}, J=15.4,5.7 \mathrm{~Hz}, 1 \mathrm{H}), 1.28(\mathrm{~s}, 12 \mathrm{H}), 1.14(\mathrm{~s}, 3 \mathrm{H}), 1.18-1.04(\mathrm{~m}, 1 \mathrm{H})$; ${ }^{13} \mathrm{C}$ NMR (151 MHz, Chloroform- $d$ ) $\delta 214.0,161.1(\mathrm{~d}, J=245.0 \mathrm{~Hz}), 134.9(\mathrm{~d}, J=2.9$ $\mathrm{Hz}), 134.4$ (d, $J=7.2 \mathrm{~Hz}), 128.7$ (d, $J=8.3 \mathrm{~Hz}), 114.9$ (d, $J=20.6 \mathrm{~Hz}), 113.4$ (d, $J=$ $21.0 \mathrm{~Hz}), 83.7$, 78.6, 53.5, 42.4, 39.8, 35.9, 25.7, 24.9, 24.7, 19.8, 14.4; HRMS: m/z (ESI) calculated $[\mathrm{M}+\mathrm{Na}]^{+}: 411.2119$, found:411.2112. $[\alpha]_{\mathrm{D}}{ }^{25}=+36.8\left(c=0.10, \mathrm{CHCl}_{3}\right)$. The enantiomeric excess of $2 \mathbf{e}$ was determined by chiral HPLC analysis compared to the corresponding racemate.

Conditions: ChiralPark IA column; hexane $/{ }^{i} \mathrm{PrOH}=90: 10$; flow rate $=1.0 \mathrm{~mL} / \mathrm{min} ; \lambda=$ $272 \mathrm{~nm} ; \mathrm{t}_{\mathrm{R} 1}$ (major) $=5.6 \mathrm{~min} ; \mathrm{t}_{\mathrm{R} 2}($ minor $)=5.3 \mathrm{~min}$.

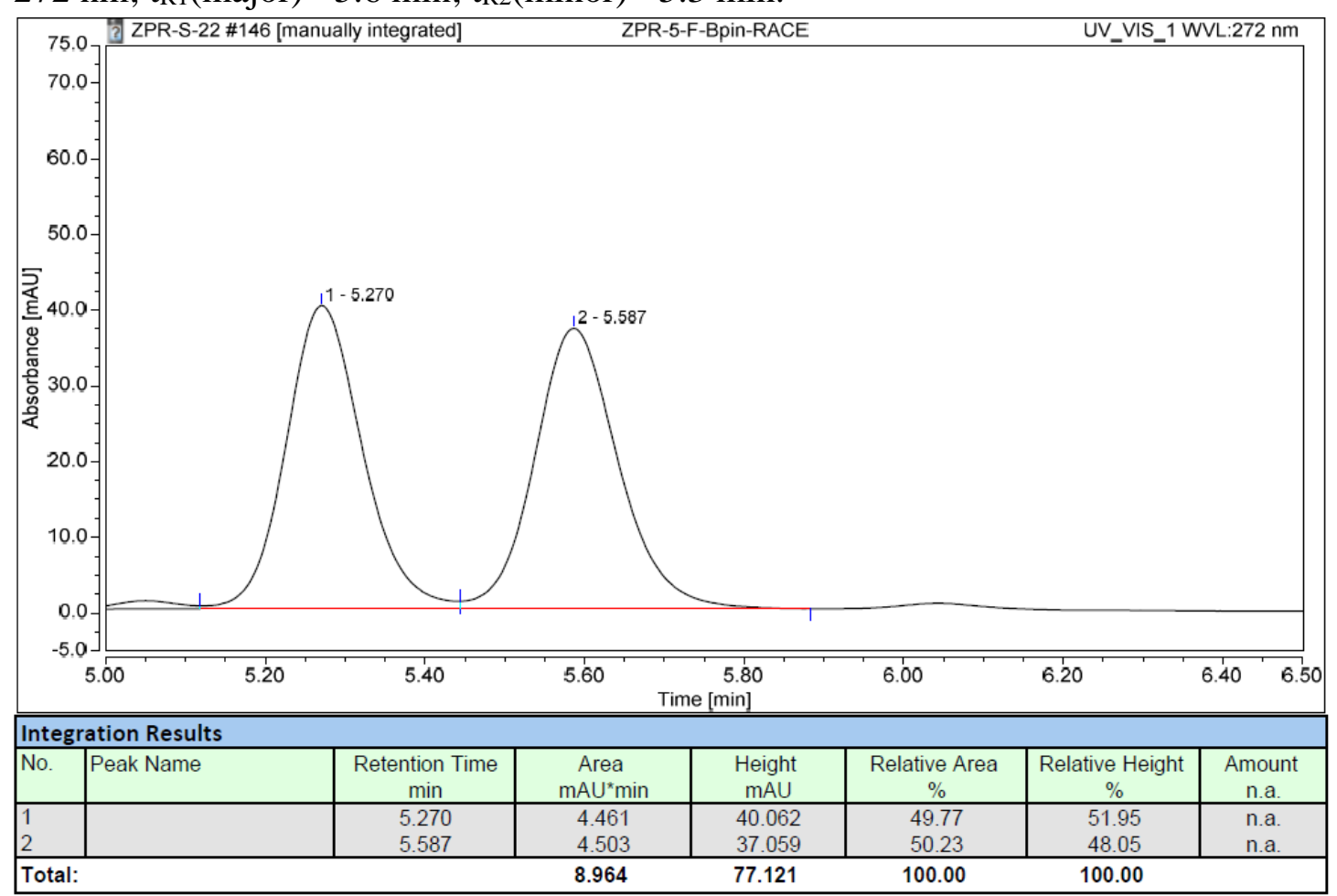




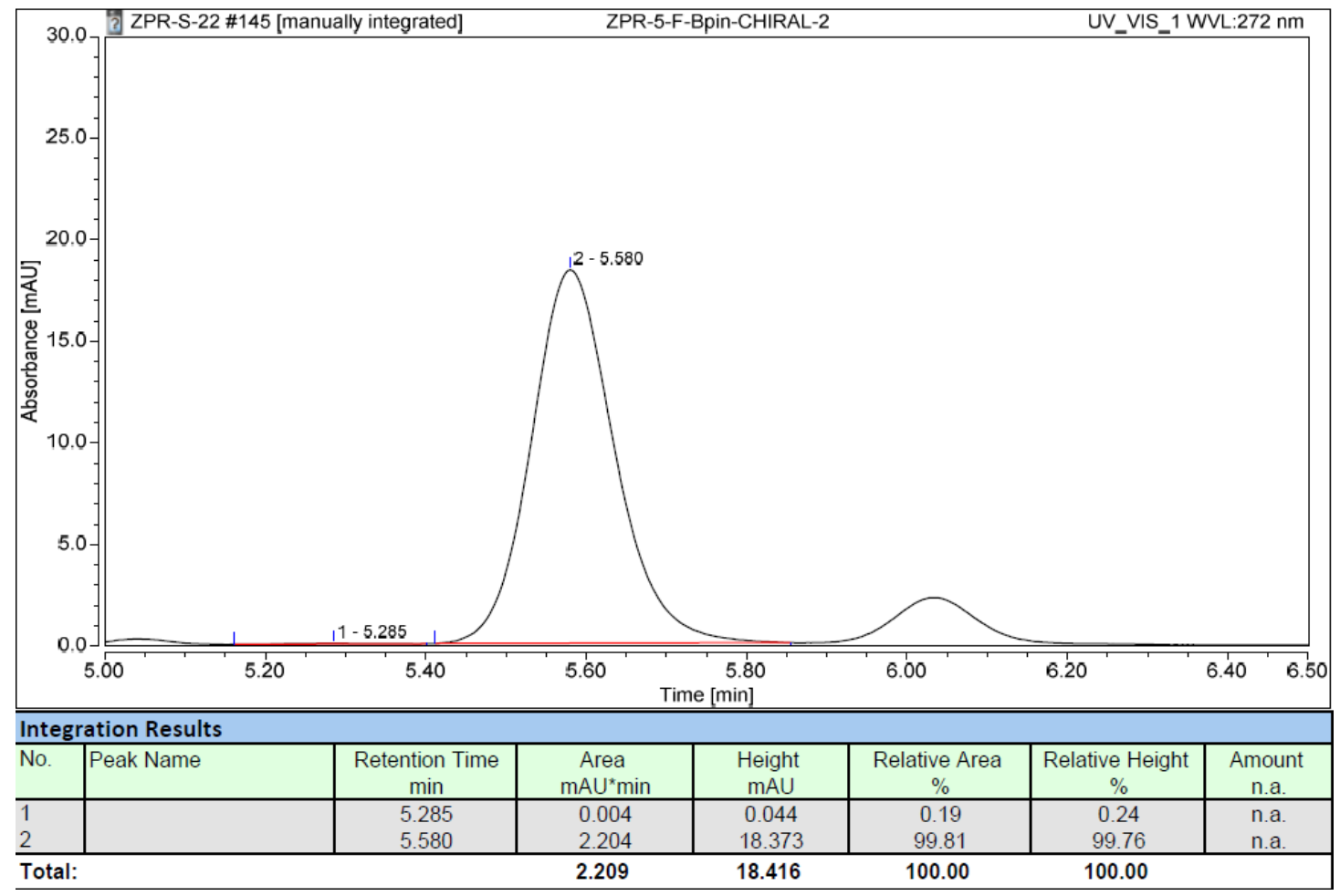<smiles>CCCC[C@H]1c2ccc(OC)cc2C[C@@]2(C)C(=O)CCC[C@@]12O</smiles>

The title compound $2 \mathbf{f}$ was synthesized according to General Procedure (SI 4.1), and it was purified by column chromatography on silica gel (58\% yield, $98 \%$ ee, $46.4 \mathrm{mg}$, white solid).

${ }^{1} \mathrm{H}$ NMR (400 MHz, Chloroform- $d$ ) $\delta 7.24$ (d, $\left.J=8.7 \mathrm{~Hz}, 1 \mathrm{H}\right), 6.76(\mathrm{dd}, J=8.7,2.7$ $\mathrm{Hz}, 1 \mathrm{H}), 6.57(\mathrm{~d}, J=2.7 \mathrm{~Hz}, 1 \mathrm{H}), 3.77(\mathrm{~s}, 3 \mathrm{H}), 3.42(\mathrm{~d}, J=17.0 \mathrm{~Hz}, 1 \mathrm{H}), 3.08(\mathrm{dd}, J=$ $10.0,5.8 \mathrm{~Hz}, 1 \mathrm{H}), 2.80-2.66(\mathrm{~m}, 1 \mathrm{H}), 2.56(\mathrm{~d}, J=17.0 \mathrm{~Hz}, 1 \mathrm{H}), 2.41-2.29(\mathrm{~m}, 2 \mathrm{H})$, 2.20-2.03 (m, 1H), 1.98-1.84 (m, 1H), 1.84-1.73 (m, 1H), 1.73-1.60 (m, 1H), $1.45(\mathrm{dd}$, $J=15.3,5.8 \mathrm{~Hz}, 1 \mathrm{H}), 1.29(\mathrm{~s}, 12 \mathrm{H}), 1.14(\mathrm{~s}, 3 \mathrm{H}), 1.08(\mathrm{dd}, J=15.3,10.0 \mathrm{~Hz}, 1 \mathrm{H}) ;{ }^{13} \mathrm{C}$ NMR $(101 \mathrm{MHz}$, Chloroform- $d$ ) $\delta 214.5,157.8,133.4,131.4,128.2,113.3,112.5,83.6$, 78.8, 55.2, 53.7, 42.3, 40.1, 36.0, 25.6, 24.9, 24.7, 19.9, 14.3; HRMS: m/z (ESI) calculated $[\mathrm{M}+\mathrm{Na}]^{+}: 423.2319$, found: $423.2316 .[\alpha]_{\mathrm{D}}^{25}=+41.0\left(c=0.10, \mathrm{CHCl}_{3}\right)$.

The enantiomeric excess of $\mathbf{2 f}$ was determined by chiral HPLC analysis compared to the corresponding racemate.

Conditions: ChiralPark IG column; hexane $/ 2 \mathrm{PrOH}=95: 5$; flow rate $=1.0 \mathrm{~mL} / \mathrm{min} ; \lambda=$ $272 \mathrm{~nm} ; \mathrm{t}_{\mathrm{R} 1}$ (major) $=19.8 \mathrm{~min} ; \mathrm{t}_{\mathrm{R} 2}($ minor $)=18.0 \mathrm{~nm}$. 

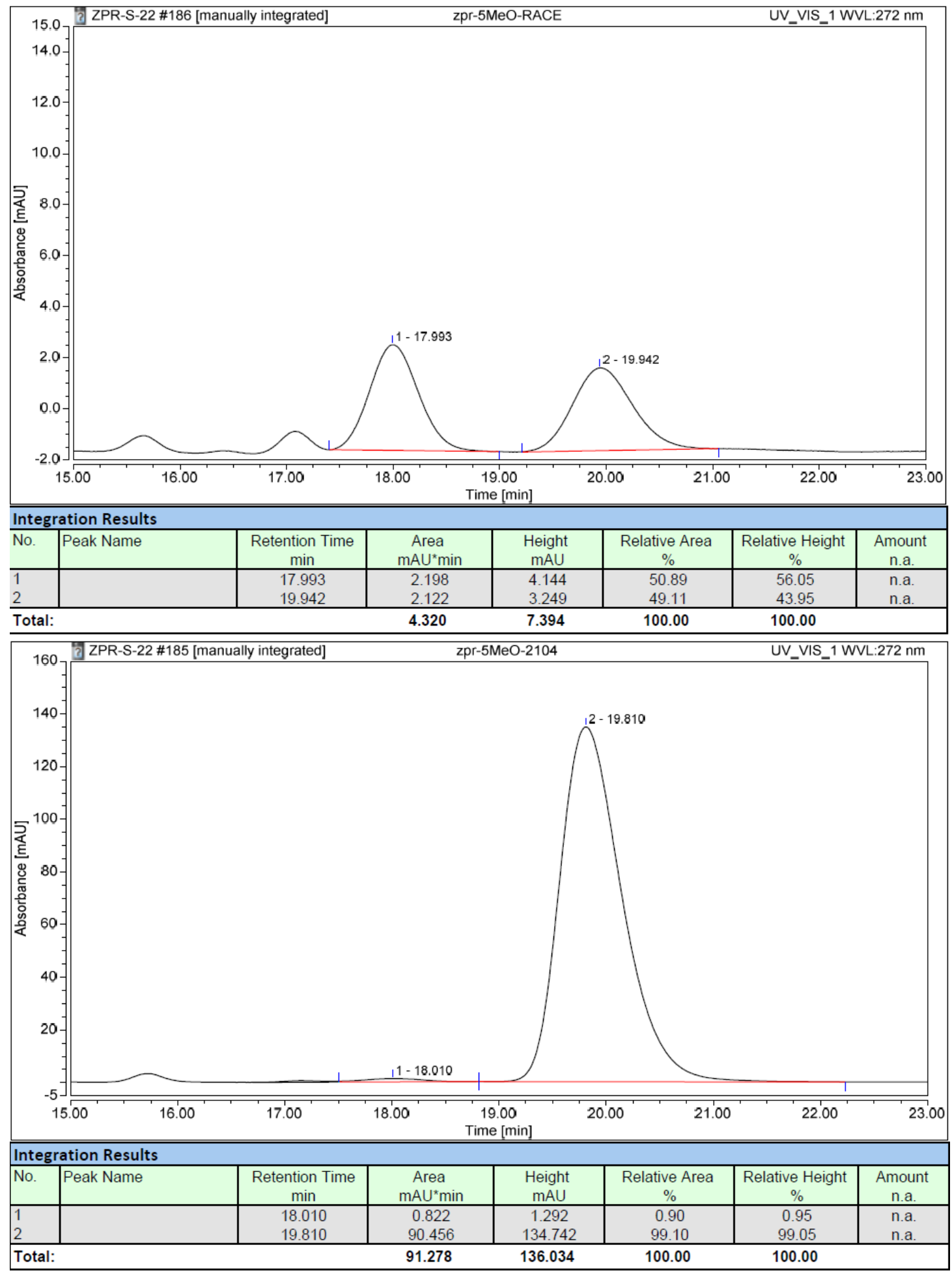<smiles>C[C@@H]1C(=O)CCC[C@]2(O)[C@H](C[Pb])c3ccc(C(F)(F)F)cc3C[C@]12C</smiles> 
The title compound $\mathbf{2 g}$ was synthesized according to General Procedure (SI 4.1), and it was purified by column chromatography on silica gel (63\% yield, 95\% ee, $55.2 \mathrm{mg}$, white solid).

${ }^{1} \mathrm{H}$ NMR (600 MHz, Chloroform- $d$ ) $\delta$ 7.47-7.41 (m, 1H), 7.31 (s, 1H), 3.48 (d, $J=17.2$ $\mathrm{Hz}, 1 \mathrm{H}), 3.21-3.13(\mathrm{~m}, 1 \mathrm{H}), 2.78-2.70(\mathrm{~m}, 1 \mathrm{H}), 2.66(\mathrm{~d}, J=17.2 \mathrm{~Hz}, 1 \mathrm{H}), 2.44(\mathrm{~s}, 1 \mathrm{H})$, 2.41-2.34 (m, 1H), 2.18-2.08 (m, 1H), 1.94-1.87 (m, 1H), 1.85-1.77 (m, 1H), 1.66-1.60 (m, 1H), $1.49(\mathrm{dd}, J=15.4,5.8 \mathrm{~Hz}, 1 \mathrm{H}), 1.29(\mathrm{~s}, 12 \mathrm{H}), 1.15(\mathrm{~s}, 3 \mathrm{H}), 1.18-1.09(\mathrm{~m}, 1 \mathrm{H})$; ${ }^{13} \mathrm{C}$ NMR (151 MHz, Chloroform- $d$ ) $\delta 213.8,143.4,133.1,128.4(\mathrm{q}, J=32.5 \mathrm{~Hz}), 127.7$, $125.7(\mathrm{q}, J=3.8 \mathrm{~Hz}), 124.1(\mathrm{q}, J=272.0 \mathrm{~Hz}), 123.1(\mathrm{q}, J=3.4 \mathrm{~Hz}), 83.8,78.4,53.4$, $42.9,39.7,35.9,25.8,24.9,24.7,19.8,14.4 ; \mathrm{HRMS}: \mathrm{m} / \mathrm{z}(\mathrm{ESI})$ calculated $[\mathrm{M}+\mathrm{H}]^{+}$: 439.2267, found: 439.2266. $[\alpha]_{\mathrm{D}}^{25}=+32.6\left(c=0.14, \mathrm{CHCl}_{3}\right)$.

The enantiomeric excess of $\mathbf{2 g}$ was determined by chiral HPLC analysis after oxidation to the corresponding alcohol compared to the corresponding racemate.

Conditions: ChiralPark IC column; hexane $/{ }^{i} \mathrm{PrOH}=90: 10$; flow rate $=1.0 \mathrm{~mL} / \mathrm{min} ; \lambda=$ $272 \mathrm{~nm} ; \mathrm{t}_{\mathrm{R} 1}($ major $)=16.1 \mathrm{~min} ; \mathrm{t}_{\mathrm{R} 2}($ minor $)=19.3 \mathrm{~min}$.

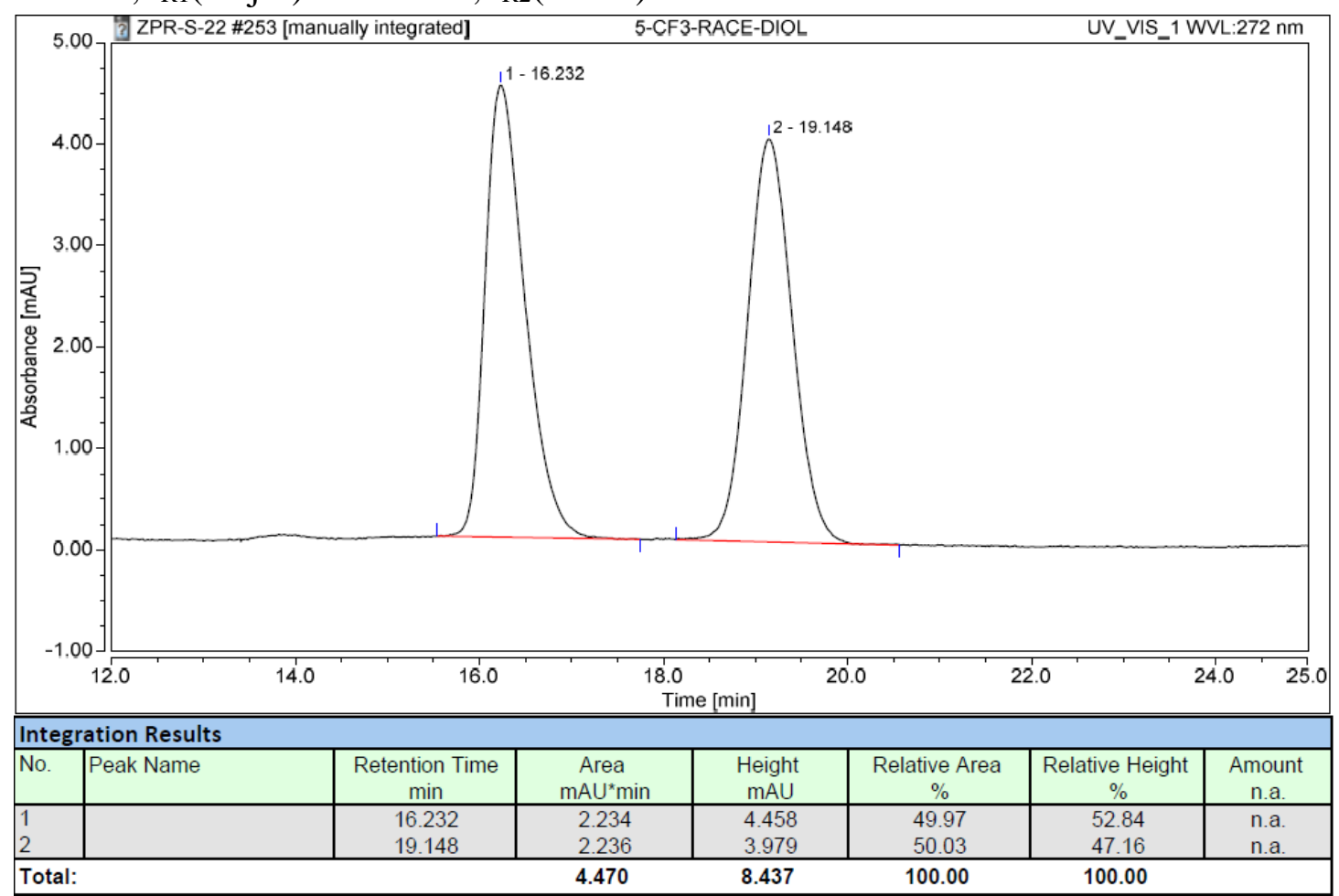




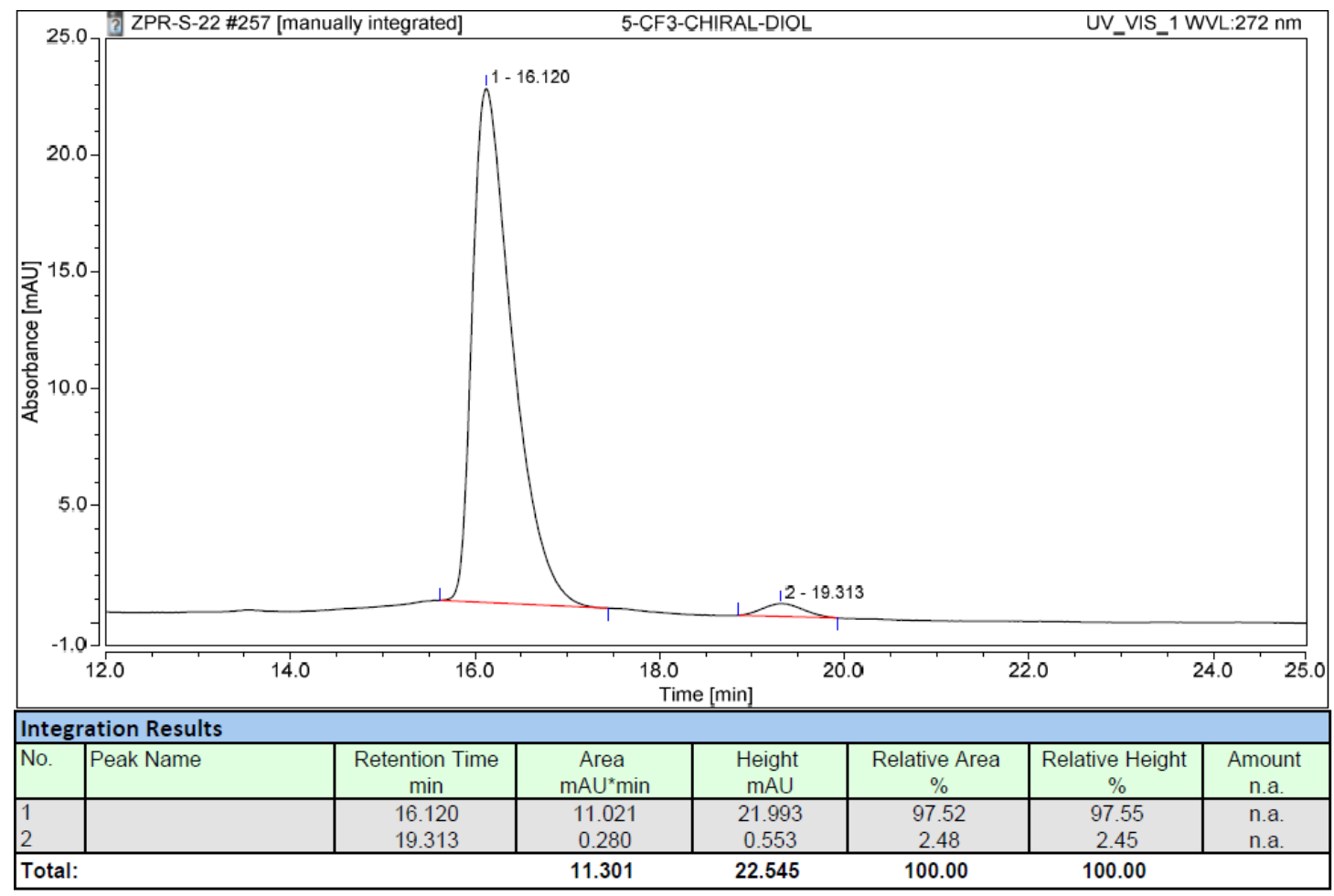<smiles>COc1ccc2c(c1)[C@H](CP)[C@]1(O)CCCC(=O)[C@@]1(C)C2</smiles>

The title compound $\mathbf{2 h}$ was synthesized according to General Procedure (SI 4.1), and it was purified by column chromatography on silica gel ( $85 \%$ yield, $99 \%$ ee, $68.0 \mathrm{mg}$, white solid).

${ }^{1} \mathrm{H}$ NMR (400 MHz, Chloroform- $d$ ) $\delta 6.96(\mathrm{~d}, J=8.4 \mathrm{~Hz}, 1 \mathrm{H}), 6.88(\mathrm{~d}, J=2.5 \mathrm{~Hz}, 1 \mathrm{H})$, $6.73(\mathrm{dd}, J=8.4,2.5 \mathrm{~Hz}, 1 \mathrm{H}), 3.79(\mathrm{~s}, 3 \mathrm{H}), 3.37$ (d, $J=16.6 \mathrm{~Hz}, 1 \mathrm{H}), 3.11(\mathrm{dd}, J=$ 10.0, $5.8 \mathrm{~Hz}, 1 \mathrm{H}), 2.82-2.67(\mathrm{~m}, 1 \mathrm{H}), 2.54(\mathrm{~d}, J=16.6 \mathrm{~Hz}, 1 \mathrm{H}), 2.42-2.27(\mathrm{~m}, 2 \mathrm{H})$, 2.19-2.02 (m, 1H), 1.94-1.84 (m, 1H), 1.84-1.77 (m, 1H), 1.70-1.61 (m, 1H), $1.45(\mathrm{dd}$, $J=15.2,5.8 \mathrm{~Hz}, 1 \mathrm{H}), 1.29(\mathrm{~s}, 12 \mathrm{H}), 1.13(\mathrm{~s}, 3 \mathrm{H}), 1.16-1.06(\mathrm{~m}, 1 \mathrm{H}) ;{ }^{13} \mathrm{C}$ NMR $(101$ MHz, Chloroform- $d$ ) $\delta 214.6,158.2,140.6,129.8,124.3,112.4,112.1,83.6,78.8,55.2$, 53.8, 43.1, 39.1, 36.0, 25.7, 24.9, 24.7, 19.8, 14.3;HRMS: m/z (ESI) calculated $[\mathrm{M}+\mathrm{H}]^{+}: 401.2499$, found: $401.2503 .[\alpha]_{\mathrm{D}}{ }^{25}=+44.4\left(c=0.16, \mathrm{CHCl}_{3}\right)$.

The enantiomeric excess of $\mathbf{2 h}$ was determined by chiral HPLC analysis compared to the corresponding racemate.

Conditions: ChiralPark IC column; hexane $/ i \mathrm{PrOH}=90: 10$; flow rate $=1.0 \mathrm{~mL} / \mathrm{min} ; \lambda=$ $272 \mathrm{~nm} ; \mathrm{t}_{\mathrm{R} 1}$ (major) $=14.1 \mathrm{~min} ; \mathrm{t}_{\mathrm{R} 2}$ (minor) $=15.8 \mathrm{~min}$. 


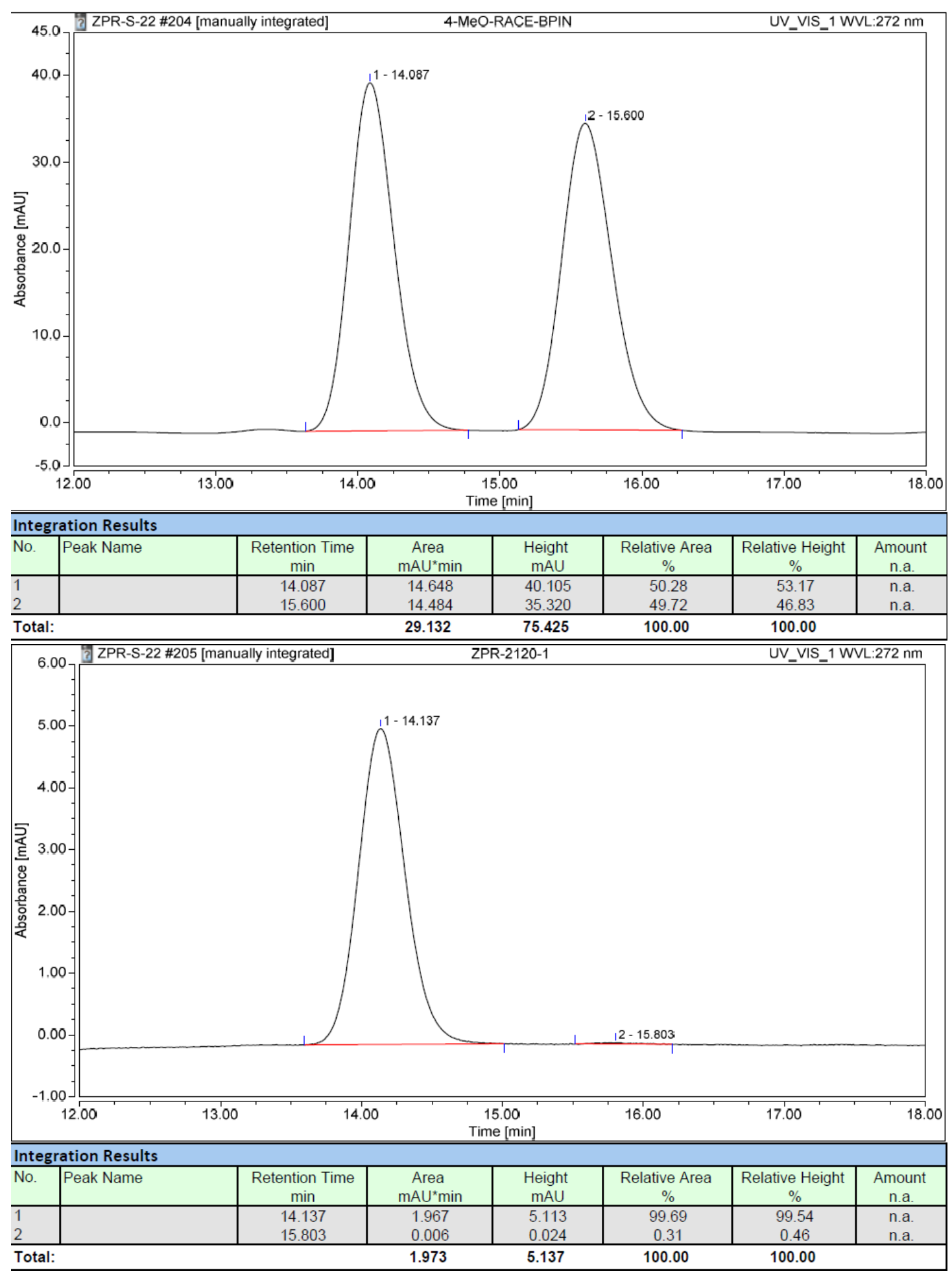


<smiles>O=C1CCC[C@]2(O)[C@H](Cc3ccccc3)c3ccccc3C[C@]12Cc1ccccc1</smiles>

The title compound 4a was synthesized according to General Procedure (SI 4.1), and it was purified by column chromatography on silica gel (58\% yield, 97\% ee, $51.7 \mathrm{mg}$, white solid).

${ }^{1} \mathrm{H}$ NMR (400 MHz, Chloroform- $d$ ) $\delta 7.38$ (d, $\left.J=7.9 \mathrm{~Hz}, 1 \mathrm{H}\right)$, 7.25-7.12 (m, 7H), 6.94 $(\mathrm{d}, J=7.6 \mathrm{~Hz}, 1 \mathrm{H}), 3.53(\mathrm{dd}, J=10.2,5.5 \mathrm{~Hz}, 1 \mathrm{H}), 3.33(\mathrm{~d}, J=17.0 \mathrm{~Hz}, 1 \mathrm{H}), 3.21-$ $3.06(\mathrm{~m}, 2 \mathrm{H}), 2.78-2.67(\mathrm{~m}, 1 \mathrm{H}), 2.60(\mathrm{~d}, J=17.0 \mathrm{~Hz}, 1 \mathrm{H}), 2.48-2.38(\mathrm{~m}, 1 \mathrm{H}), 2.24(\mathrm{~s}$, $1 \mathrm{H}), 2.18-2.04(\mathrm{~m}, 1 \mathrm{H}), 1.91-1.81(\mathrm{~m}, 1 \mathrm{H}), 1.81-1.72(\mathrm{~m}, 1 \mathrm{H}), 1.71-1.64(\mathrm{~m}, 1 \mathrm{H}), 1.49$ $(\mathrm{dd}, J=15.4,5.5 \mathrm{~Hz}, 1 \mathrm{H}), 1.25(\mathrm{~s}, 6 \mathrm{H}), 1.19(\mathrm{~s}, 6 \mathrm{H}), 1.11(\mathrm{dd}, J=15.4,10.2 \mathrm{~Hz}, 1 \mathrm{H})$; ${ }^{13} \mathrm{C}$ NMR (101 MHz, Chloroform- $d$ ) $\delta$ 214.0, 139.5, 138.9, 132.4, 131.8, 128.9, 128.0, 127.2, 126.5, 126.2, 126.1, 83.5, 80.3, 58.9, 43.4, 37.9, 36.8, 34.4, 27.0, 24.80, 24.76, 19.6; HRMS: $\mathrm{m} / \mathrm{z}(\mathrm{ESI})$ calculated $[\mathrm{M}+\mathrm{K}]^{+}: 485.2265$, found: $485.2262 .[\alpha]_{\mathrm{D}}{ }^{25}=-4.2$ $\left(c=0.18, \mathrm{CHCl}_{3}\right)$.

The enantiomeric excess of $\mathbf{4 a}$ was determined by chiral HPLC analysis after oxidation to the corresponding alcohol compared to the corresponding racemate.

Conditions: ChiralPark IF column; hexane $/{ }^{i} \mathrm{PrOH}=90: 10$; flow rate $=1.0 \mathrm{~mL} / \mathrm{min} ; \lambda=$ $272 \mathrm{~nm} ; \mathrm{t}_{\mathrm{R} 1}$ (major) $=36.1 \mathrm{~min} ; \mathrm{t}_{\mathrm{R} 2}$ (minor) $=23.7 \mathrm{~min}$.

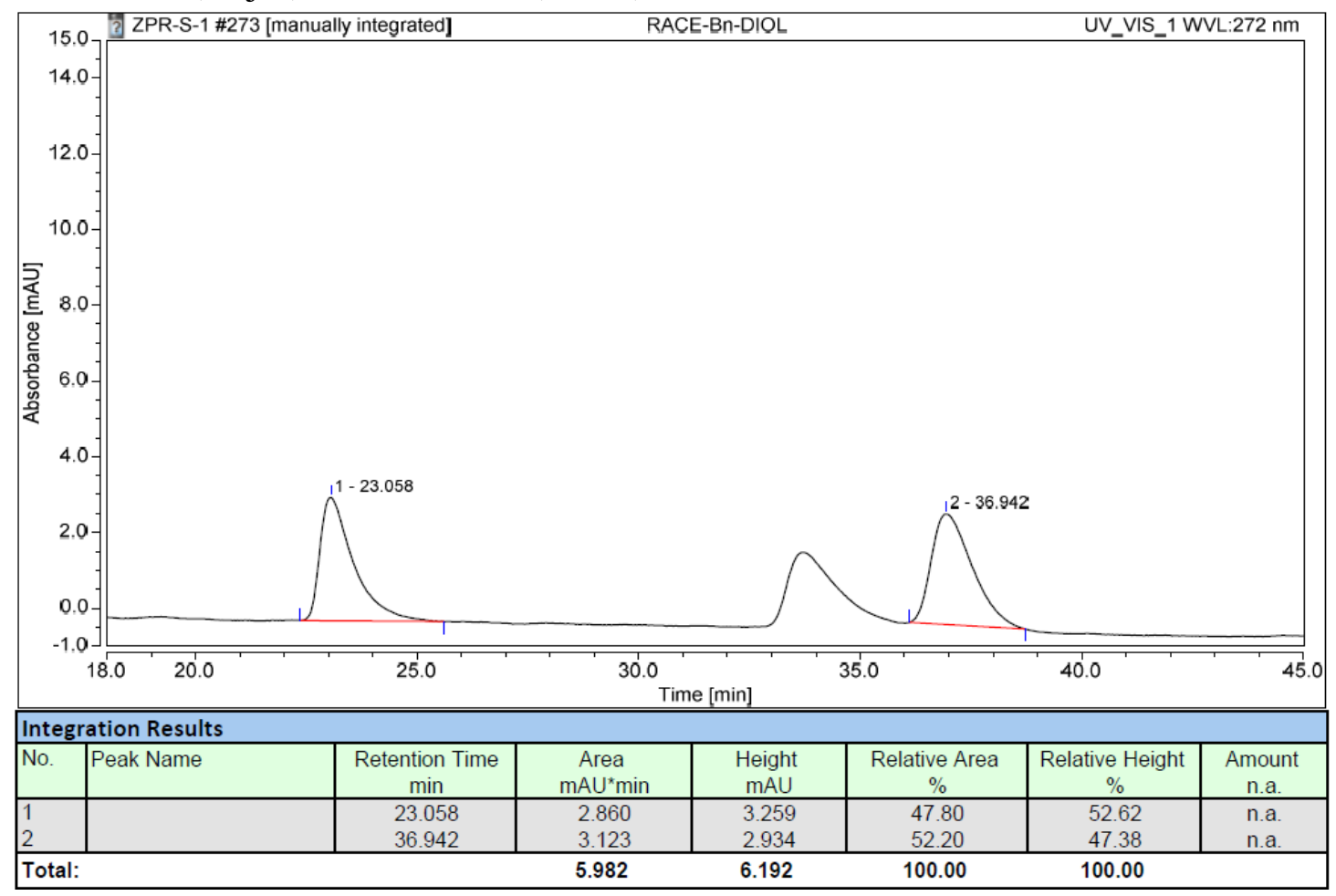




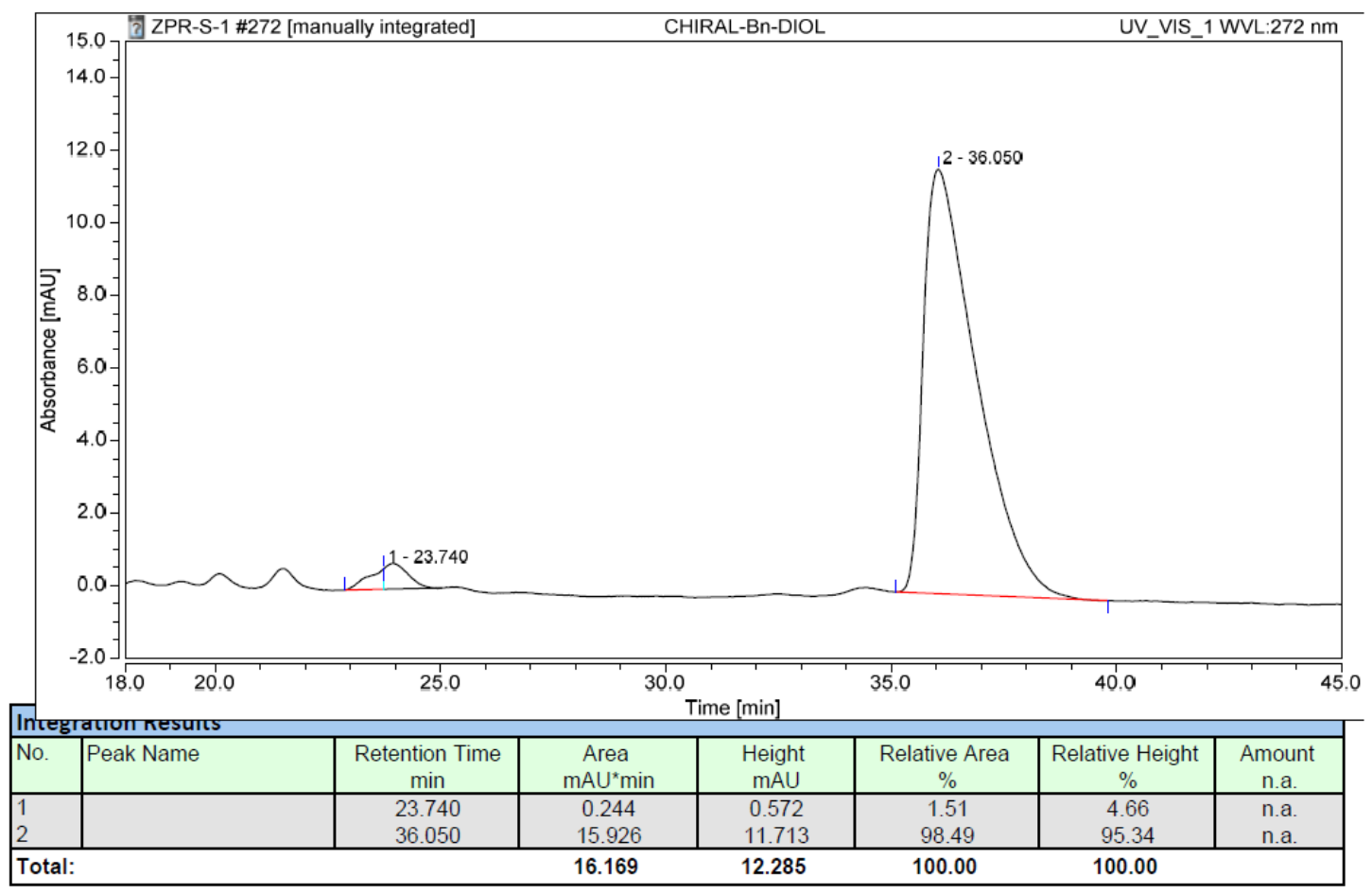<smiles>O=C1CCC[C@]2(Cc3ccccc3)Cc3ccccc3[C@H](CP)[C@@]12O</smiles>

The title compound $\mathbf{4 b}$ was synthesized according to General Procedure (SI 4.1), and it was purified by column chromatography on silica gel (69\% yield, $82 \% e e, 71.0 \mathrm{mg}$, white solid).

${ }^{1} \mathrm{H}$ NMR (600 MHz, Chloroform- $d$ ) $\delta$ 7.43-7.33 (m, 3H), 7.30-7.22 (m, 1H), 7.18-7.11 $(\mathrm{m}, 1 \mathrm{H}), 6.83(\mathrm{~d}, J=7.6 \mathrm{~Hz}, 1 \mathrm{H}), 3.52-3.44(\mathrm{~m}, 2 \mathrm{H}), 3.33(\mathrm{~d}, J=16.7 \mathrm{~Hz}, 1 \mathrm{H}), 2.93(\mathrm{~s}$, $1 \mathrm{H}), 2.79(\mathrm{~d}, J=13.9 \mathrm{~Hz}, 1 \mathrm{H}), 2.77-2.70(\mathrm{~m}, 1 \mathrm{H}), 2.44(\mathrm{~d}, J=16.7 \mathrm{~Hz}, 1 \mathrm{H}), 2.40-2.32$ $(\mathrm{m}, 1 \mathrm{H}), 2.19-2.07(\mathrm{~m}, 1 \mathrm{H}), 1.96-1.88(\mathrm{~m}, 1 \mathrm{H}), 1.81(\mathrm{~d}, J=10.5 \mathrm{~Hz}, 1 \mathrm{H}), 1.60(\mathrm{dd}, J=$ $15.8,4.8 \mathrm{~Hz}, 1 \mathrm{H}), 1.31-1.25(\mathrm{~m}, 12 \mathrm{H}), 1.15(\mathrm{dd}, J=15.8,10.8 \mathrm{~Hz}, 1 \mathrm{H}) ;{ }^{13} \mathrm{C} \mathrm{NMR}(151$ MHz, Chloroform- $d$ ) $\delta 213.6,140.0,139.6,135.2,131.9,129.7$ (q, $J=31.7 \mathrm{~Hz}), 129.0$, $128.3(\mathrm{q}, J=3.6 \mathrm{~Hz}), 127.9,127.4,126.8,126.4,124.2(\mathrm{q}, J=271.8 \mathrm{~Hz}), 122.7$ (q, $J=$ $3.8 \mathrm{~Hz}), 83.9,80.5,58.8,43.6,36.8,36.4,33.1,28.0,24.7,24.7,20.3$; HRMS: m/z (ESI) calculated $\left[\mathrm{M}+\mathrm{NH}_{4}\right]^{+}: 532.2846$, found: $532.2840 .[\alpha]_{\mathrm{D}}{ }^{25}=+21.8\left(c=0.22, \mathrm{CHCl}_{3}\right)$.

The enantiomeric excess of $\mathbf{4 b}$ was determined by chiral HPLC analysis compared to the corresponding racemate.

Conditions: ChiralPark Ic column; hexane $/{ }^{i} \mathrm{PrOH}=90: 10$; flow rate $=0.5 \mathrm{~mL} / \mathrm{min} ; \lambda=$ $272 \mathrm{~nm} ; \mathrm{t}_{\mathrm{R} 1}($ major $)=10.6 \mathrm{~min} ; \mathrm{t}_{\mathrm{R} 2}($ minor $)=11.4 \mathrm{~min}$. 

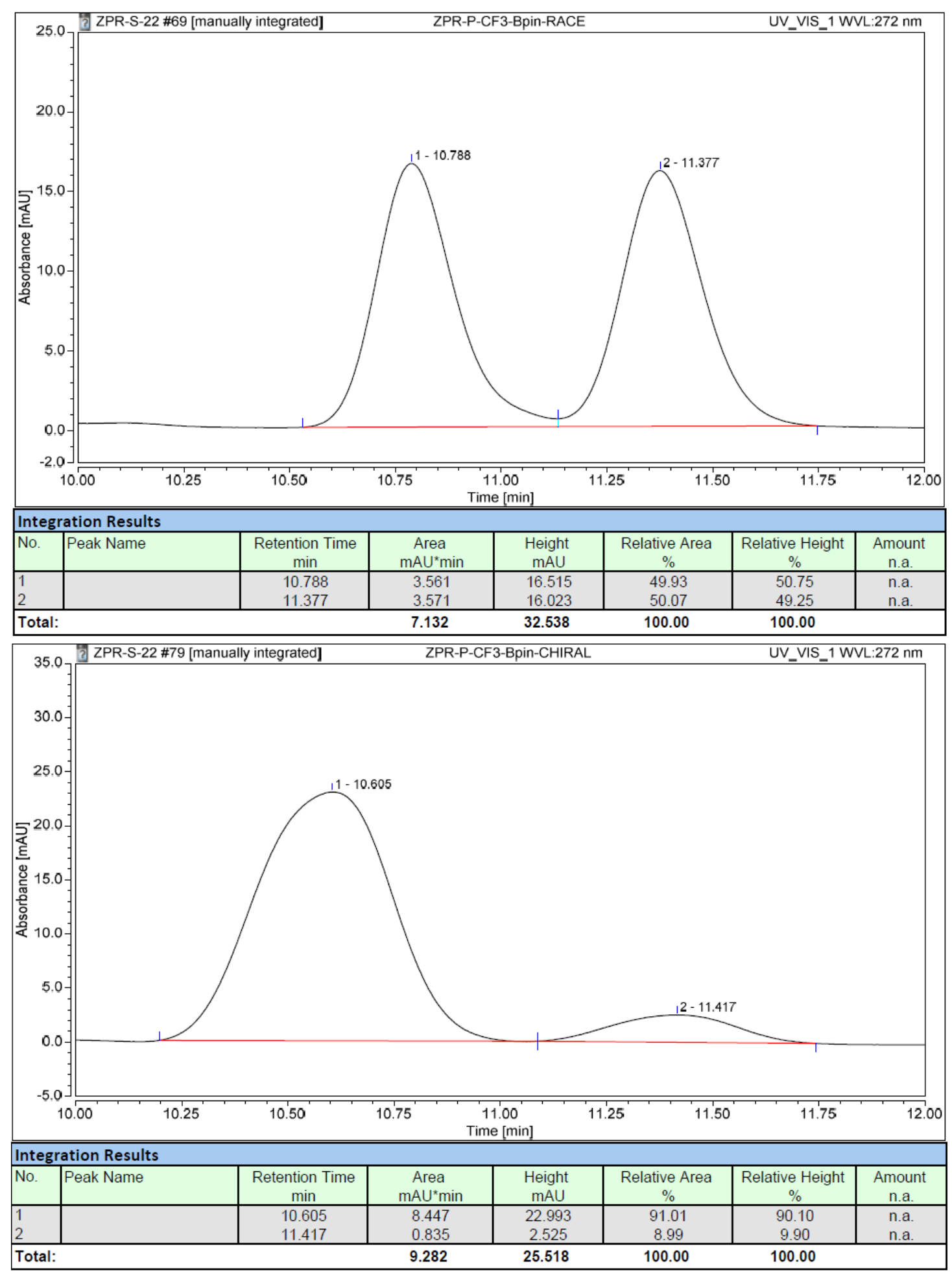
<smiles>COc1ccc(C[C@]23Cc4ccccc4[C@@H](Cc4ccccc4)[C@@]2(O)CCCC3=O)cc1</smiles>

The title compound $\mathbf{4 c}$ was synthesized according to General Procedure (SI 4.1), and it was purified by column chromatography on silica gel (65\% yield, 97\% ee, $61.9 \mathrm{mg}$, white solid).

${ }^{1} \mathrm{H}$ NMR (400 MHz, Chloroform- $d$ ) $\delta 7.38(\mathrm{~d}, J=7.9 \mathrm{~Hz}, 1 \mathrm{H}), 7.24(\mathrm{dd}, J=7.9,6.8$ $\mathrm{Hz}, 1 \mathrm{H}), 7.16(\mathrm{t}, J=8.6 \mathrm{~Hz}, 3 \mathrm{H}), 6.97(\mathrm{~d}, J=7.6 \mathrm{~Hz}, 1 \mathrm{H}), 6.74(\mathrm{~d}, J=8.2 \mathrm{~Hz}, 2 \mathrm{H})$, $3.76(\mathrm{~s}, 3 \mathrm{H}), 3.51(\mathrm{dd}, J=10.1,5.6 \mathrm{~Hz}, 1 \mathrm{H}), 3.32(\mathrm{~d}, J=17.0 \mathrm{~Hz}, 1 \mathrm{H}), 3.15-3.01(\mathrm{~m}$, $2 \mathrm{H}), 2.78-2.66(\mathrm{~m}, 1 \mathrm{H}), 2.61(\mathrm{~d}, J=17.0 \mathrm{~Hz}, 1 \mathrm{H}), 2.47-2.37(\mathrm{~m}, 1 \mathrm{H}), 2.19(\mathrm{~s}, 1 \mathrm{H})$, 2.17-2.04 (m, 1H), 1.89-1.80 (m, 1H), 1.81-1.72 (m, 1H), 1.65-1.57 (m, 1H), $1.47(\mathrm{dd}$, $J=15.4,5.6 \mathrm{~Hz}, 1 \mathrm{H}), 1.26-1.16(\mathrm{~m}, 12 \mathrm{H}), 1.10(\mathrm{dd}, J=15.4,10.1 \mathrm{~Hz}, 1 \mathrm{H}) ;{ }^{13} \mathrm{C} \mathrm{NMR}$ (101 MHz, Chloroform- $d$ ) $\delta$ 214.1, 158.0, 139.6, 132.8, 132.4, 130.7, 128.9, 127.1, 126.5, 126.1, 113.5, 83.4, 80.3, 58.8, 55.1, 43.4, 38.2, 36.8, 33.8, 26.9, 24.9, 24.8, 24.6, 19.5; HRMS: $\mathrm{m} / \mathrm{z}(\mathrm{ESI})$ calculated $[\mathrm{M}+\mathrm{Na}]^{+}: 499.2632$, found: $499.2636 .[\alpha]_{\mathrm{D}}^{25}=-2.8$ $\left(c=0.22, \mathrm{CHCl}_{3}\right)$.

The enantiomeric excess of $\mathbf{4} \mathbf{c}$ was determined by chiral HPLC analysis after oxidation to the corresponding alcohol compared to the corresponding racemate.

Conditions: ChiralPak ID column; hexane $/{ }^{i} \mathrm{PrOH}=90: 10$; flow rate $=1.0 \mathrm{~mL} / \mathrm{min} ; \lambda=$ $272 \mathrm{~nm} ; \mathrm{t}_{\mathrm{R} 1}$ (major) $=43.1 \mathrm{~min} ; \mathrm{t}_{\mathrm{R} 2}$ (minor) $=31.5 \mathrm{~min}$.

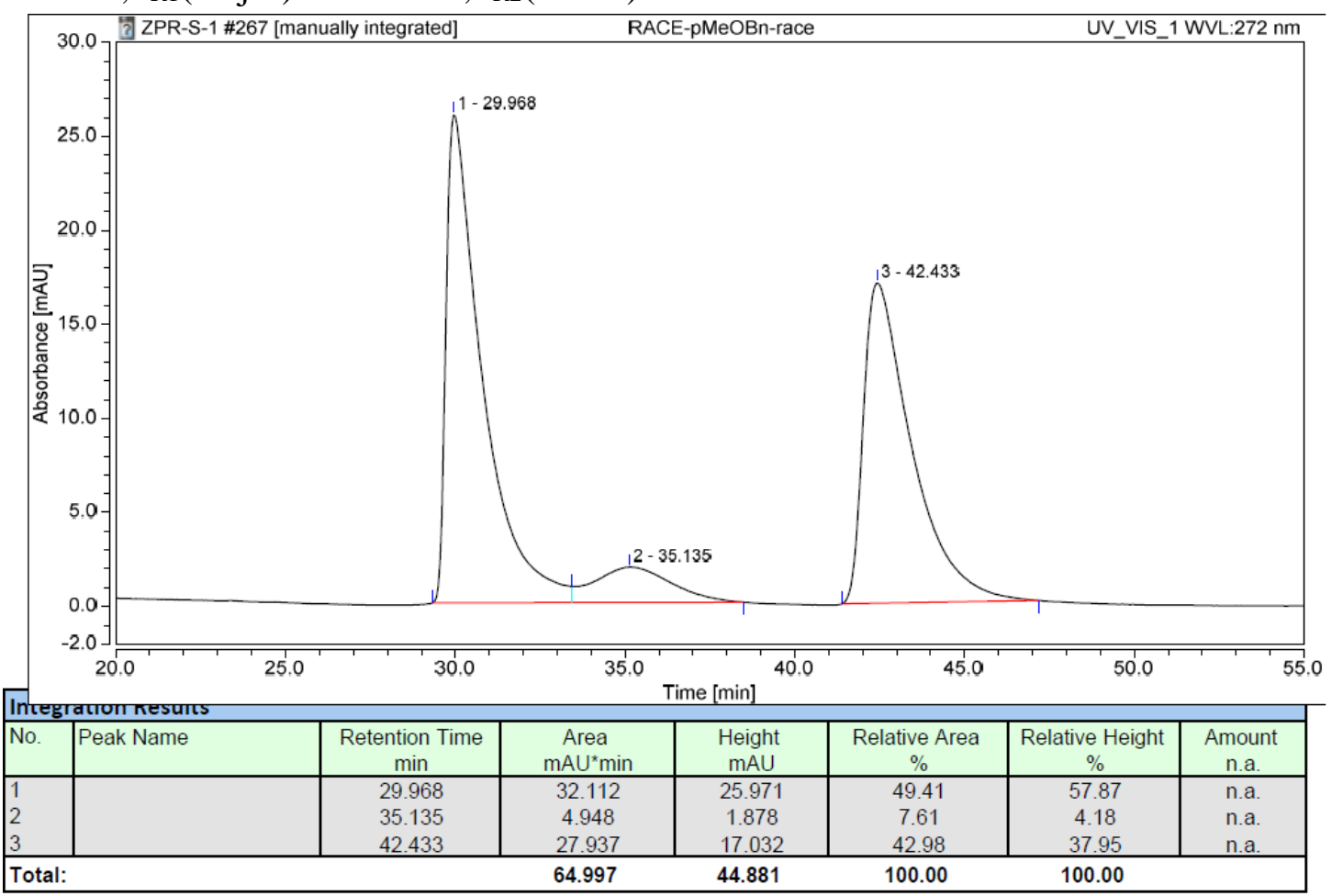




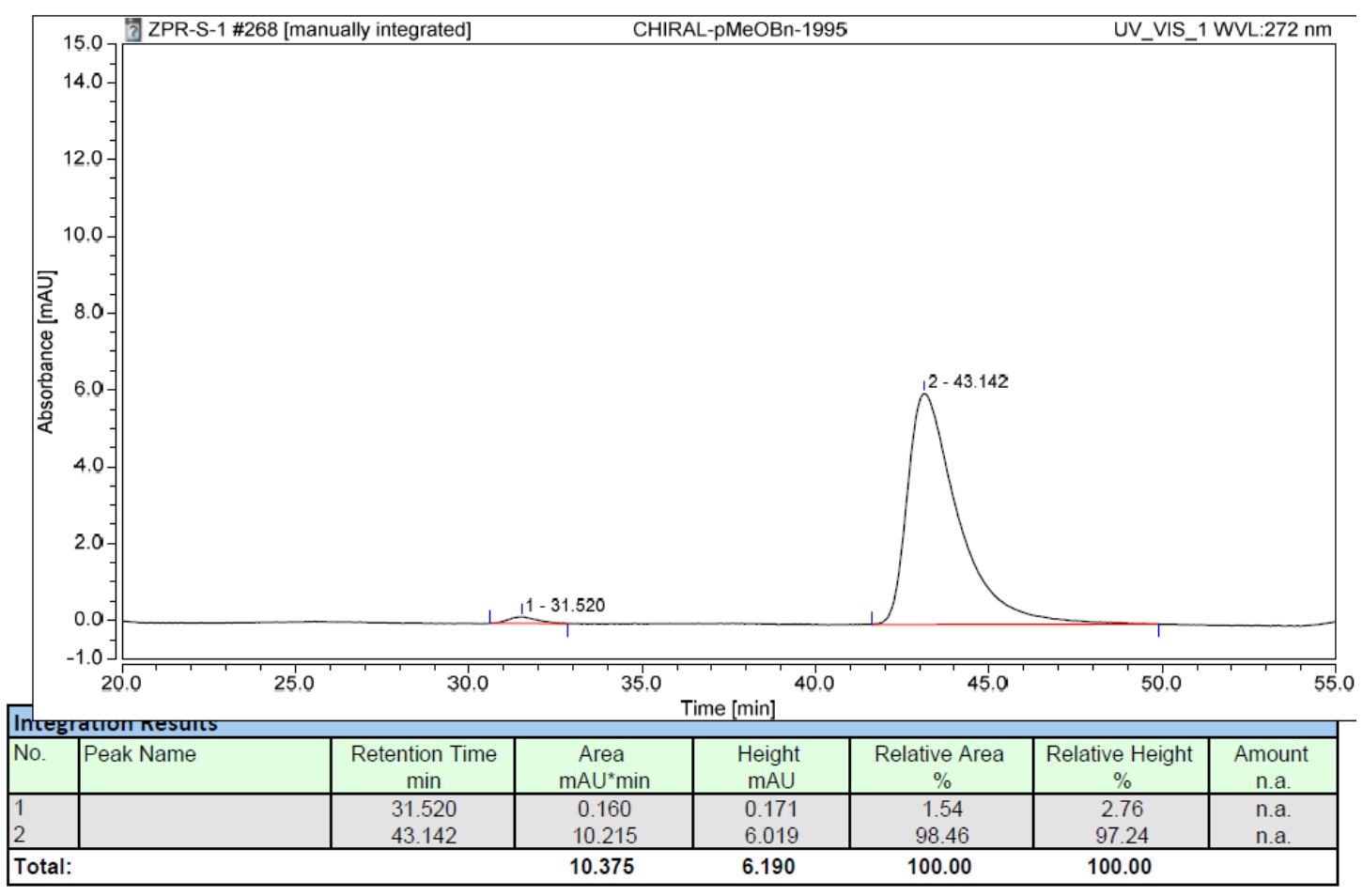<smiles>O=C1CCC[C@]2(O)[C@H](Cc3ccccc3)c3ccccc3C[C@]12Cc1ccccc1</smiles>

The title compound $\mathbf{4 d}$ was synthesized according to General Procedure (SI 4.2), and it was purified by column chromatography on silica gel ( $84 \%$ yield, $90 \%$ ee, $88.1 \mathrm{mg}$, white solid).

${ }^{1} \mathrm{H}$ NMR (600 MHz, Chloroform- $d$ ) $\delta 7.38$ (d, $\left.J=7.8 \mathrm{~Hz}, 1 \mathrm{H}\right), 7.29-7.2(\mathrm{~m}, 3 \mathrm{H}), 7.15$ $(\mathrm{dd}, J=7.8,7.2 \mathrm{~Hz}, 1 \mathrm{H}), 7.01(\mathrm{~d}, J=8.4 \mathrm{~Hz}, 2 \mathrm{H}), 6.91(\mathrm{~d}, J=7.8 \mathrm{~Hz}, 1 \mathrm{H}), 3.47(\mathrm{dd}, J$ $=10.2,5.0 \mathrm{~Hz}, 1 \mathrm{H}), 3.32(\mathrm{~d}, J=17.0 \mathrm{~Hz}, 1 \mathrm{H}), 3.22(\mathrm{~d}, J=14.0 \mathrm{~Hz}, 1 \mathrm{H}), 2.90(\mathrm{~d}, J=$ $14.0 \mathrm{~Hz}, 1 \mathrm{H}), 2.78-2.67(\mathrm{~m}, 1 \mathrm{H}), 2.52(\mathrm{~d}, J=17.0 \mathrm{~Hz}, 1 \mathrm{H}), 2.47$ (s, 1H), 2.42-2.34 (m, $1 \mathrm{H}), 2.16-2.06(\mathrm{~m}, 1 \mathrm{H}), 1.92-1.84(\mathrm{~m}, 1 \mathrm{H}), 1.79-1.73(\mathrm{~m}, 1 \mathrm{H}), 1.73-1.69(\mathrm{~m}, 1 \mathrm{H}), 1.53$ $(\mathrm{dd}, J=15.6,5.0 \mathrm{~Hz}, 1 \mathrm{H}), 1.26(\mathrm{~s}, 6 \mathrm{H}), 1.22(\mathrm{~s}, 6 \mathrm{H}), 1.11(\mathrm{dd}, J=15.6,10.2 \mathrm{~Hz}, 1 \mathrm{H})$; ${ }^{13} \mathrm{C}$ NMR (151 MHz, Chloroform- $d$ ) $\delta 213.8,139.5,138.0,133.5,132.1,130.8,128.9$, 127.3, 126.6, 126.2, 120.1, 83.6, 80.4, 58.9, 43.4, 37.2, 36.8, 33.4, 27.4, 24.8 , 24.7, 19.9; HRMS: $\mathrm{m} / \mathrm{z}(\mathrm{ESI})$ calculated $\left[\mathrm{M}+\mathrm{NH}_{4}\right]^{+}: 542.2077$, found: $542.2045 .[\alpha]_{\mathrm{D}}{ }^{25}=-$ $16.1\left(c=0.28, \mathrm{CHCl}_{3}\right)$.

The enantiomeric excess of $\mathbf{4 d}$ was determined by chiral HPLC analysis compared to the corresponding racemate. 
Conditions: ChiralPak IC column; hexane $/ \mathrm{PrOH}=90: 10$; flow rate $=0.6 \mathrm{~mL} / \mathrm{min} ; \lambda=$ $272 \mathrm{~nm} ; \mathrm{t}_{\mathrm{R} 1}($ major $)=14.7 \mathrm{~min} ; \mathrm{t}_{\mathrm{R} 2}($ minor $)=16.3 \mathrm{~min}$.
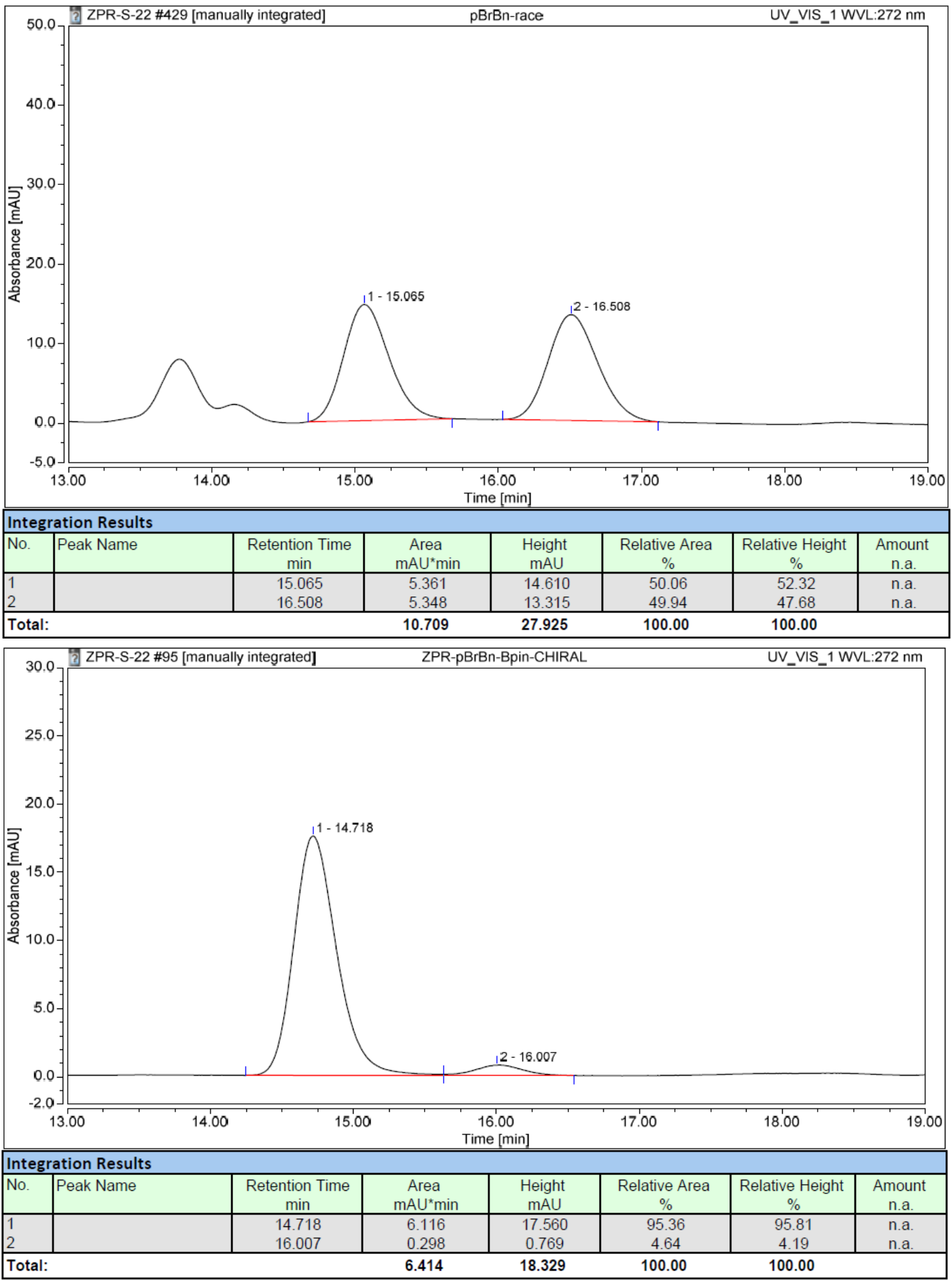
<smiles>O=C1CCC[C@]2(O)[C@H](Cc3ccccc3)c3ccccc3C[C@]12Cc1ccccc1</smiles>

The title compound 4e was synthesized according to General Procedure (SI 4.2), and it was purified by column chromatography on silica gel (72\% yield, 96\% ee, $69.1 \mathrm{mg}$, white solid).

${ }^{1} \mathrm{H}$ NMR (400 MHz, Chloroform- $d$ ) $\delta 7.39$ (d, $\left.J=7.8 \mathrm{~Hz}, 1 \mathrm{H}\right)$, 7.26-7.22 (m, 1H), 7.16 $(\mathrm{dd}, J=7.6,7.2 \mathrm{~Hz}, 1 \mathrm{H}), 7.13-7.03(\mathrm{~m}, 3 \mathrm{H}), 7.00(\mathrm{~d}, J=7.5 \mathrm{~Hz}, 1 \mathrm{H}), 6.89(\mathrm{~d}, J=7.8$ $\mathrm{Hz}, 1 \mathrm{H}), 3.47$ (dd, $J=10.2,5.2 \mathrm{~Hz}, 1 \mathrm{H}), 3.37-3.25(\mathrm{~m}, 2 \mathrm{H}), 2.86(\mathrm{~d}, J=13.9 \mathrm{~Hz}, 1 \mathrm{H})$, 2.79-2.68 (m, 1H), $2.63(\mathrm{~s}, 1 \mathrm{H}), 2.50(\mathrm{~d}, J=17.0 \mathrm{~Hz}, 1 \mathrm{H}), 2.44-2.34(\mathrm{~m}, 1 \mathrm{H}), 2.20-2.06$ (m, 1H), 1.94-1.86 (m, 1H), 1.79-1.72 (m, 1H), 1.56 (dd, $J=15.8,5.2 \mathrm{~Hz}, 1 \mathrm{H}), 1.30-$ $1.21(\mathrm{~m}, 12 \mathrm{H}), 1.13(\mathrm{dd}, J=15.8,10.2 \mathrm{~Hz}, 1 \mathrm{H}) ;{ }^{13} \mathrm{C}$ NMR $(101 \mathrm{MHz}$, Chloroform-d) $\delta 213.6,141.1,139.5,133.5,132.0,131.7,129.9,128.9,128.9,127.3,126.6,126.3$, 126.2, 83.7, 80.4, 58.9, 43.5, 37.0, 36.7, 33.5, 27.6, 24.8, 24.7, 20.0; HRMS: m/z (ESI) calculated $[\mathrm{M}+\mathrm{K}]^{+}: 519.1876$, found: $519.1874 .[\alpha]_{\mathrm{D}}{ }^{25}=-4.3\left(c=0.22, \mathrm{CHCl}_{3}\right)$.

The enantiomeric excess of $\mathbf{4} \mathbf{e}$ was determined by chiral HPLC analysis compared to the corresponding racemate.

Conditions: ChiralPak IF column; hexane $/ \mathrm{PrOH}=98: 2$; flow rate $=1.0 \mathrm{~mL} / \mathrm{min} ; \lambda=$ $272 \mathrm{~nm} ; \mathrm{t}_{\mathrm{R} 1}$ (major) $=19.1 \mathrm{~min} ; \mathrm{t}_{\mathrm{R} 2}($ minor $)=15.9 \mathrm{~min}$. 


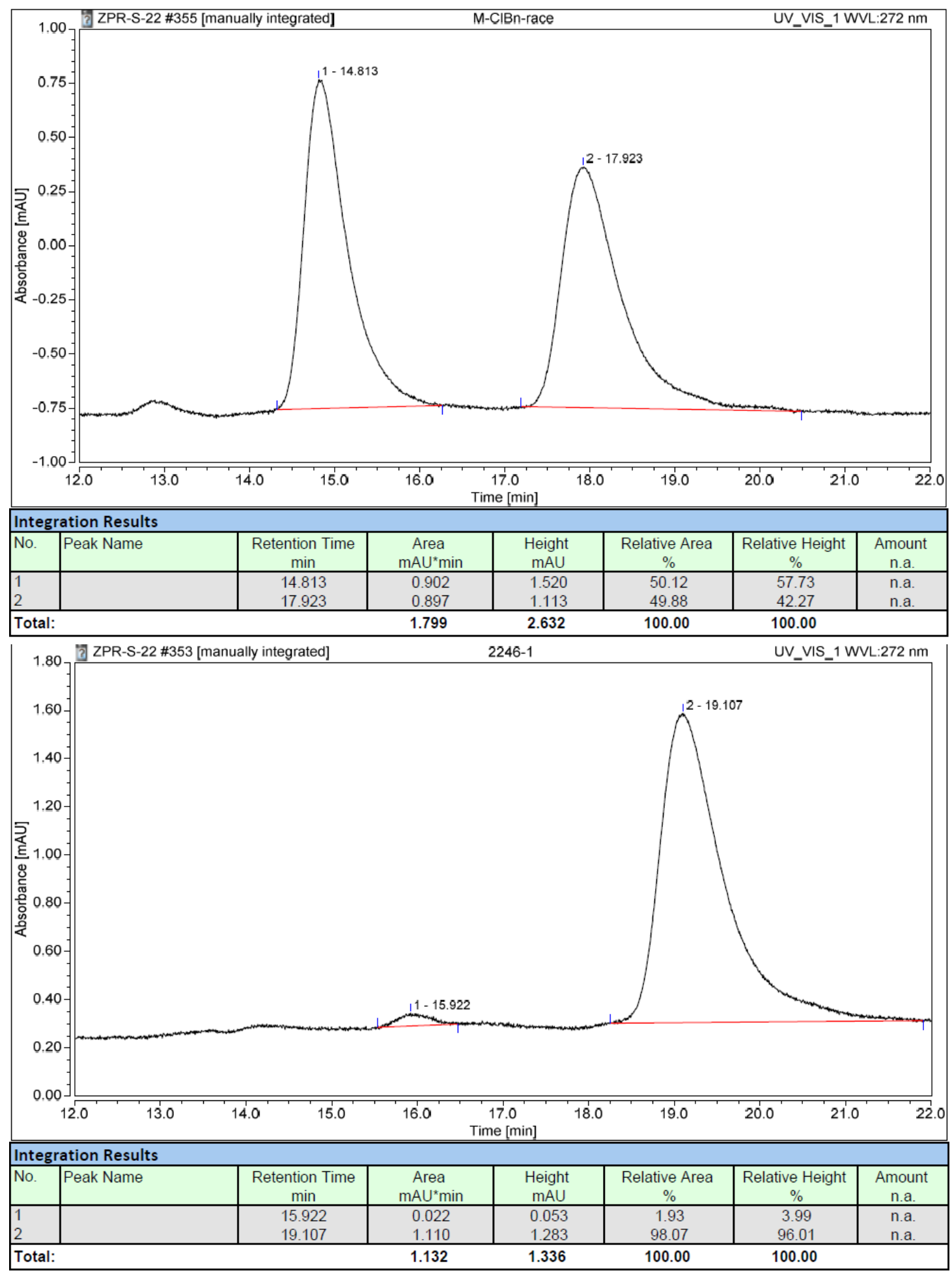


<smiles>O=C1CCC[C@]2(O)[C@H](Cc3ccccc3)c3ccccc3C[C@]12Cc1ccccc1</smiles>

The title compound $\mathbf{4 f}$ was synthesized according to General Procedure (SI 4.1), and it was purified by column chromatography on silica gel (62\% yield, 96\% ee, $62.5 \mathrm{mg}$, white solid).

${ }^{1} \mathrm{H}$ NMR (400 MHz, Chloroform- $d$ ) $\delta 7.36(\mathrm{~d}, J=8.0 \mathrm{~Hz}, 1 \mathrm{H}), 7.23$ (dd, $J=8.0,7.6$ $\mathrm{Hz}, 1 \mathrm{H}), 7.16(\mathrm{dd}, J=7.6,7.2 \mathrm{~Hz}, 1 \mathrm{H}), 6.97(\mathrm{~d}, J=7.2 \mathrm{~Hz}, 1 \mathrm{H}), 6.84(\mathrm{~s}, 1 \mathrm{H}), 6.70-$ $6.61(\mathrm{~m}, 2 \mathrm{H}), 4.20(\mathrm{~s}, 4 \mathrm{H}), 3.51(\mathrm{dd}, J=10.3,5.9 \mathrm{~Hz}, 1 \mathrm{H}), 3.32(\mathrm{~d}, J=17.1 \mathrm{~Hz}, 1 \mathrm{H})$, 3.12-2.92 (m, 2H), 2.77-2.58 (m, 2H), 2.46-2.38 (m, 1H), $2.21(\mathrm{~s}, 1 \mathrm{H}), 2.16-2.04(\mathrm{~m}$, $1 \mathrm{H}), 1.88-1.80(\mathrm{~m}, 1 \mathrm{H}), 1.79-1.72(\mathrm{~m}, 1 \mathrm{H}), 1.67-1.56(\mathrm{~m}, 1 \mathrm{H}), 1.45(\mathrm{dd}, J=15.4,5.9$ $\mathrm{Hz}, 1 \mathrm{H}), 1.28-1.19(\mathrm{~m}, 12 \mathrm{H}), 1.11(\mathrm{dd}, J=15.4,10.3 \mathrm{~Hz}, 1 \mathrm{H}) ;{ }^{13} \mathrm{C}$ NMR $(101 \mathrm{MHz}$, Chloroform- $d$ ) $\delta 213.9,143.0,142.0,139.5,132.3,131.9,128.9,127.1,126.5,126.1$, 124.8, 120.5, 116.6, 83.4, 80.2, 64.3, 64.2, 58.8, 43.3, 38.2, 36.7, 34.0, 26.8, 24.8, 24.8, 19.4; HRMS: $\mathrm{m} / \mathrm{z}(\mathrm{ESI})$ calculated $[\mathrm{M}+\mathrm{Na}]^{+}: 527.2581$, found: $527.2584 .[\alpha]_{\mathrm{D}}^{25}=-17.6$ $\left(c=0.11, \mathrm{CHCl}_{3}\right)$.

The enantiomeric excess of $\mathbf{4 f}$ was determined by chiral HPLC analysis compared to the corresponding racemate.

Conditions: ChiralPak ID column; hexane $/{ }^{i} \mathrm{PrOH}=90: 10$; flow rate $=1.0 \mathrm{~mL} / \mathrm{min} ; \lambda=$ $272 \mathrm{~nm} ; \mathrm{t}_{\mathrm{R} 1}$ (major) $=20.0 \mathrm{~min} ; \mathrm{t}_{\mathrm{R} 2}($ minor $)=17.0 \mathrm{~min}$.

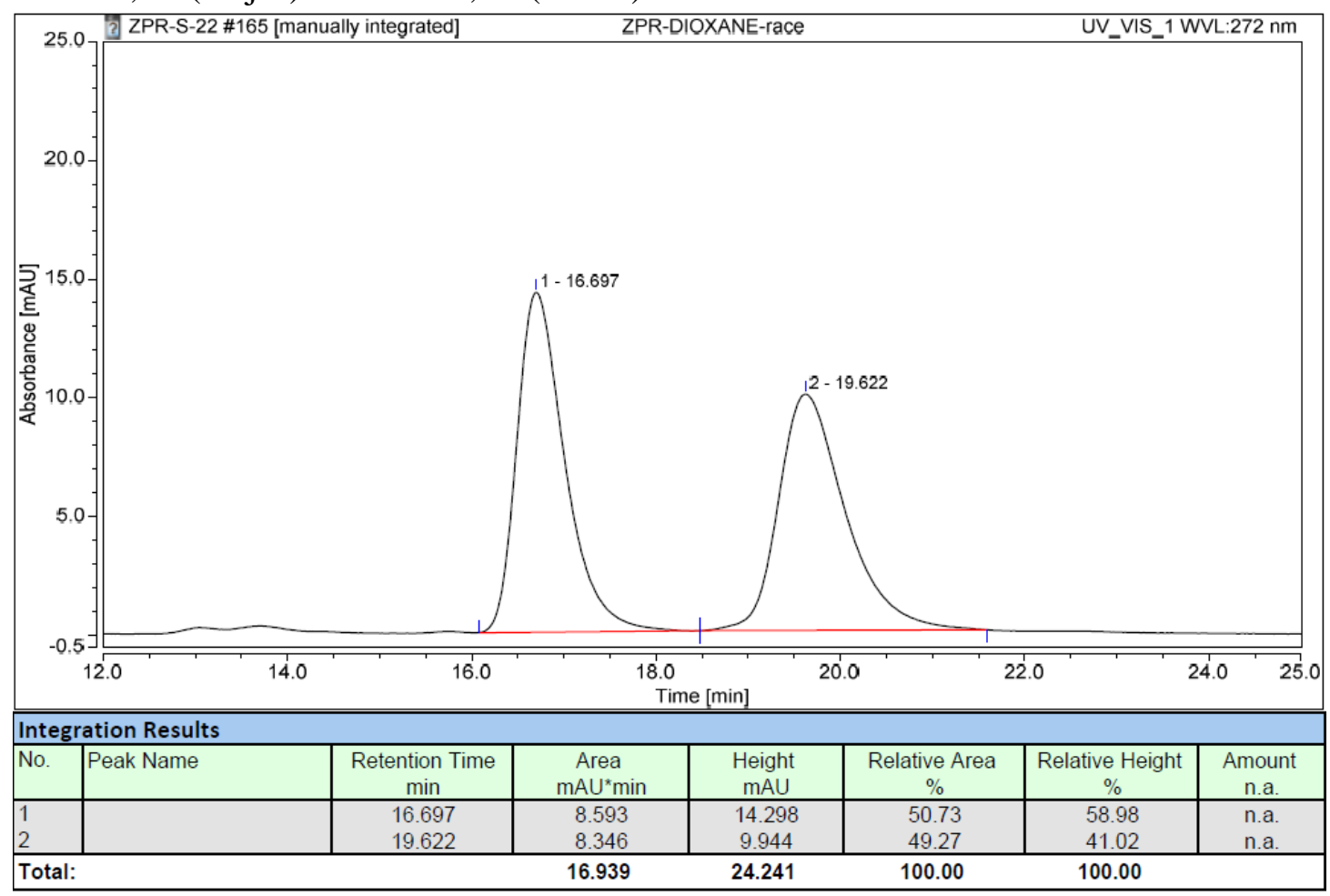




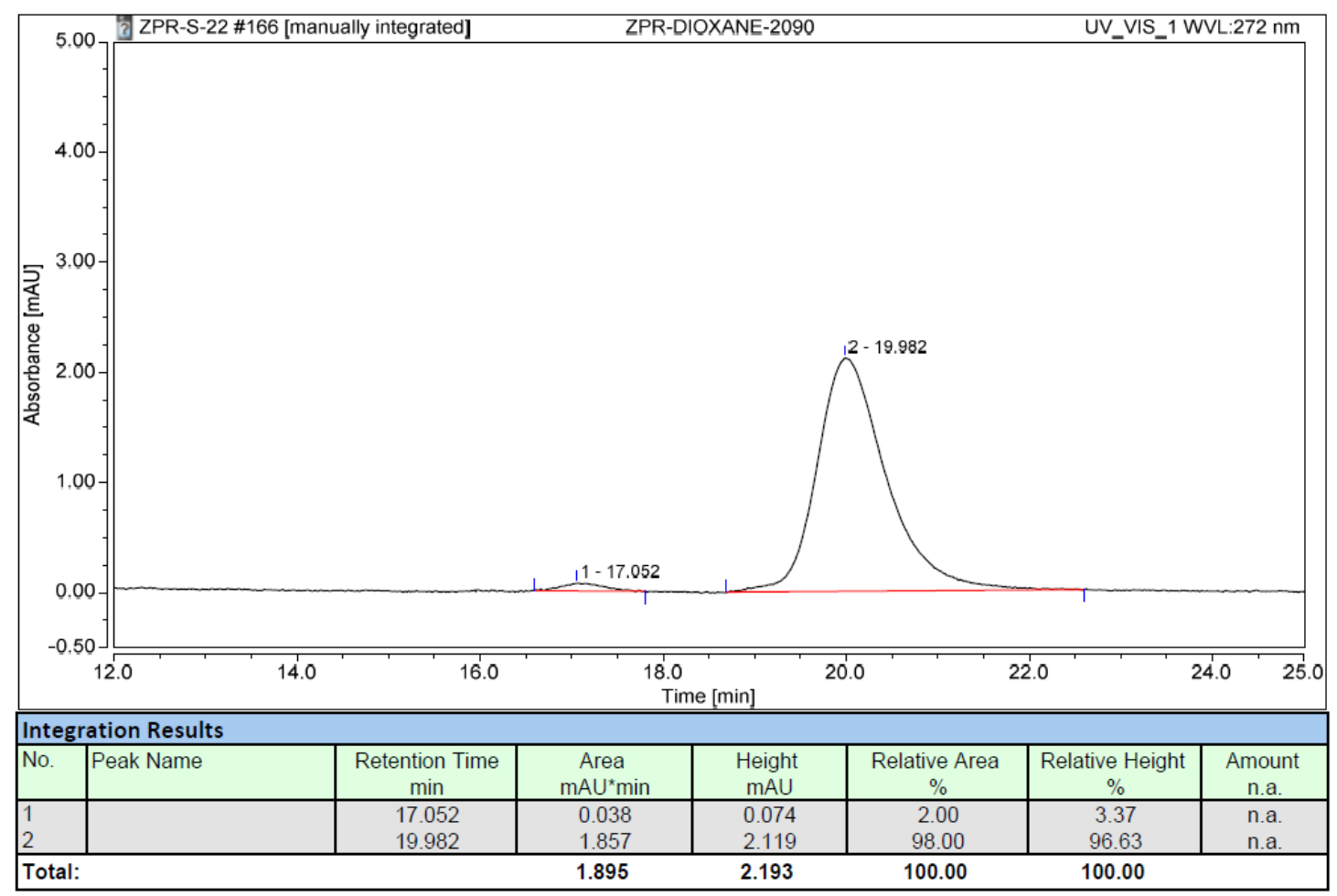<smiles>O=C1CCC[C@]2(O)[C@H](Cc3ccccc3)c3ccccc3C[C@]12Cc1ccccc1</smiles>

$4 \mathrm{~g}$

The title compound $\mathbf{4 g}$ was synthesized according to General Procedure (SI 4.1), and it was purified by column chromatography on silica gel (64\% yield, 99\% ee, $55.8 \mathrm{mg}$, white solid).

${ }^{1} \mathrm{H}$ NMR (400 MHz, Chloroform- $d$ ) $\delta 7.38(\mathrm{~d}, J=7.8 \mathrm{~Hz}, 1 \mathrm{H}), 7.27(\mathrm{~d}, J=1.8 \mathrm{~Hz}, 1 \mathrm{H})$, $7.22(\mathrm{dd}, J=7.8,7.2 \mathrm{~Hz}, 1 \mathrm{H}), 7.16(\mathrm{dd}, J=7.4,7.2 \mathrm{~Hz}, 1 \mathrm{H}), 7.02(\mathrm{~d}, J=7.4 \mathrm{~Hz}, 1 \mathrm{H})$, $6.27(\mathrm{dd}, \mathrm{J}=2.8,1.8 \mathrm{~Hz}, 1 \mathrm{H}), 6.20(\mathrm{~d}, J=2.8 \mathrm{~Hz}, 1 \mathrm{H}), 3.52(\mathrm{dd}, J=10.7,5.4 \mathrm{~Hz}, 1 \mathrm{H})$, $3.38(\mathrm{~d}, J=17.0 \mathrm{~Hz}, 1 \mathrm{H}), 3.18(\mathrm{~d}, J=15.1 \mathrm{~Hz}, 1 \mathrm{H}), 3.02$ (d, $J=15.1 \mathrm{~Hz}, 1 \mathrm{H}), 2.71-$ $2.57(\mathrm{~m}, 2 \mathrm{H}), 2.47-2.38(\mathrm{~m}, 1 \mathrm{H}), 2.34(\mathrm{~s}, 1 \mathrm{H}), 2.17-2.02(\mathrm{~m}, 1 \mathrm{H}), 1.87-1.73(\mathrm{~m}, 2 \mathrm{H})$, 1.63-1.56 (m, 1H), $1.51(\mathrm{dd}, J=15.2,5.4 \mathrm{~Hz}, 1 \mathrm{H}), 1.27-1.20(\mathrm{~m}, 12 \mathrm{H}), 1.10(\mathrm{dd}, J=$ 15.2, $10.7 \mathrm{~Hz}, 1 \mathrm{H}) ;{ }^{13} \mathrm{C}$ NMR (101 MHz, Chloroform- $d$ ) $\delta 212.9,153.4,141.0,139.3$, 132.1, 128.8, 127.0, 126.5, 126.1, 110.7, 109.6, 83.3, 79.2, 58.1, 38.9, 36.3, 28.2, 26.6, 24.9, 24.8, 19.0; HRMS: m/z (ESI) calculated $[\mathrm{M}+\mathrm{H}]^{+}: 437.2499$, found: 437.2490 . $[\alpha]_{\mathrm{D}}^{25}=+9.5\left(c=0.14, \mathrm{CHCl}_{3}\right)$.

The enantiomeric excess of $\mathbf{4 g}$ was determined by chiral HPLC analysis compared to the corresponding racemate.

Conditions: ChiralPak IC column; hexane $/ \mathrm{PrOH}=90: 10$; flow rate $=1.0 \mathrm{~mL} / \mathrm{min} ; \lambda=$ $272 \mathrm{~nm} ; \mathrm{t}_{\mathrm{R} 1}$ (major) $=11.4 \mathrm{~min} ; \mathrm{t}_{\mathrm{R} 2}($ minor $)=12.1 \mathrm{~min}$. 

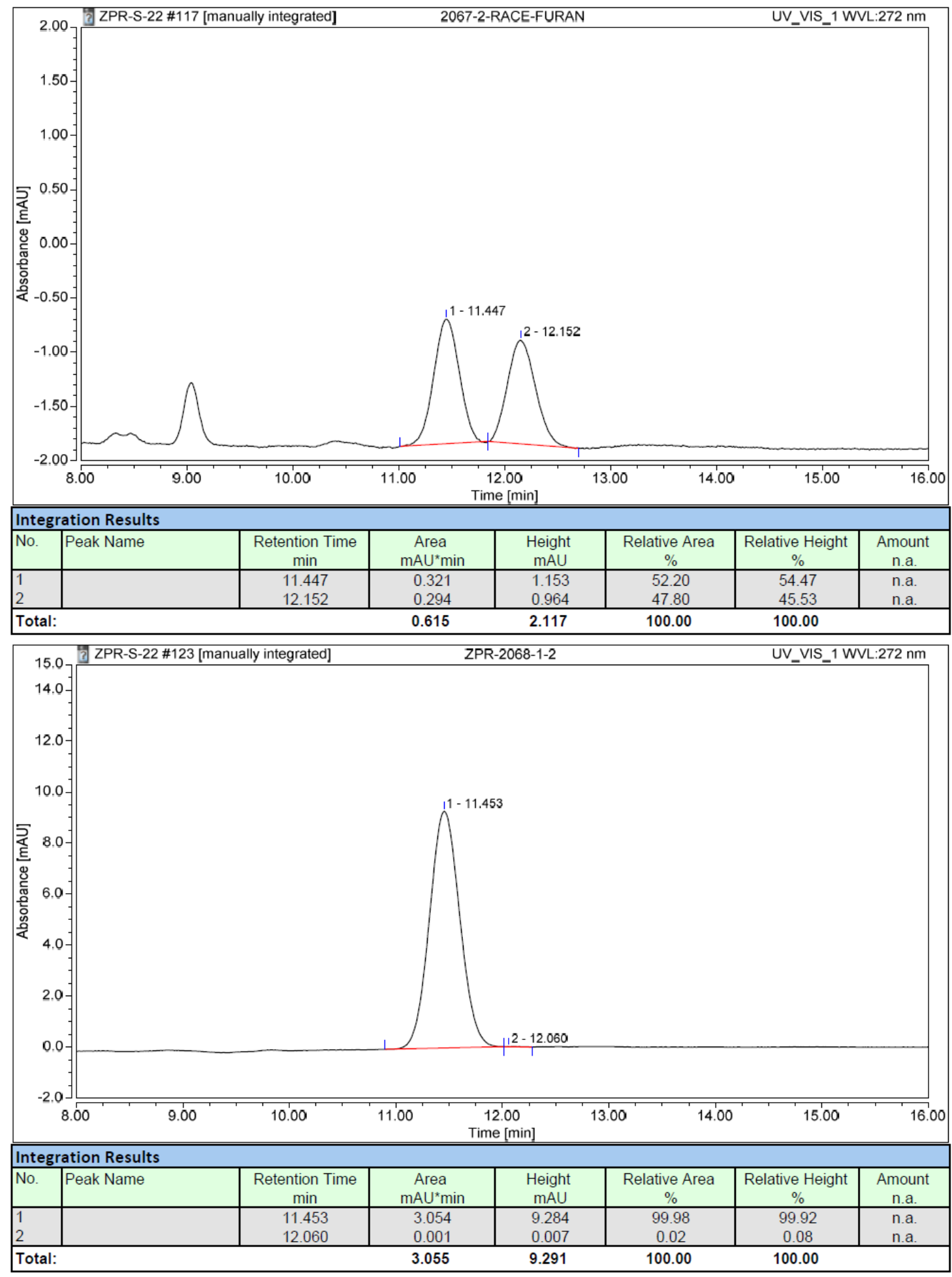
<smiles>O=C1CCC[C@]2(O)[C@H](Cc3ccccc3)c3ccccc3C[C@]12Cc1ccccc1</smiles>

4h

The title compound $4 \mathrm{~h}$ was synthesized according to General Procedure (SI 4.1), and it was purified by column chromatography on silica gel (92\% yield, 99\% ee, $82.3 \mathrm{mg}$, white solid).

${ }^{1} \mathrm{H}$ NMR (400 MHz, Chloroform- $d$ ) $\delta 7.39$ (d, $\left.J=7.9 \mathrm{~Hz}, 1 \mathrm{H}\right), 7.26-7.21(\mathrm{~m}, 1 \mathrm{H}), 7.21$ $7.12(\mathrm{~m}, 2 \mathrm{H}), 6.99$ (d, $J=7.5 \mathrm{~Hz}, 1 \mathrm{H}), 6.83(\mathrm{dd}, J=5.2,3.4 \mathrm{~Hz}, 1 \mathrm{H}), 6.69$ (d, $J=3.4$ $\mathrm{Hz}, 1 \mathrm{H}), 3.53$ (dd, $J=10.4,5.4 \mathrm{~Hz}, 1 \mathrm{H}), 3.42-3.21(\mathrm{~m}, 3 \mathrm{H}), 2.81-2.69$ (m, 1H), 2.63 (d, $J=17.1 \mathrm{~Hz}, 1 \mathrm{H}), 2.49-2.39(\mathrm{~m}, 1 \mathrm{H}), 2.36(\mathrm{~s}, 1 \mathrm{H}), 2.19-2.07(\mathrm{~m}, 1 \mathrm{H}), 1.92-1.82(\mathrm{~m}$, $1 \mathrm{H}), 1.81-1.73(\mathrm{~m}, 1 \mathrm{H}), 1.72-1.65(\mathrm{~m}, 1 \mathrm{H}), 1.50(\mathrm{dd}, J=15.4,5.4 \mathrm{~Hz}, 1 \mathrm{H}), 1.27-1.16$ $(\mathrm{m}, 12 \mathrm{H}), 1.10(\mathrm{dd}, J=15.4,10.4 \mathrm{~Hz}, 1 \mathrm{H}) ;{ }^{13} \mathrm{C}$ NMR (151 MHz, Chloroform- $d$ ) $\delta 213.7$, 141.2, 139.3, 131.7, 129.0, 128.5, 127.2, 126.6, 126.2, 126.1 , 125.9, 83.4, 80.2, 58.5, 43.0, 38.1, 36.3, 29.8, 26.8, 24.8, 24.7, 19.6; HRMS: m/z (ESI) calculated $[\mathrm{M}+\mathrm{H}]^{+}: 453.2271$, found: $453.2272 .[\alpha]_{\mathrm{D}}{ }^{25}=+9.3\left(c=0.30, \mathrm{CHCl}_{3}\right)$.

The enantiomeric excess of $\mathbf{4 h}$ was determined by chiral HPLC analysis compared to the corresponding racemate.

Conditions: ChiralPak IA column; hexane $/{ }^{i} \mathrm{PrOH}=90: 10$; flow rate $=0.8 \mathrm{~mL} / \mathrm{min} ; \lambda=$ $272 \mathrm{~nm} ; \mathrm{t}_{\mathrm{R} 1}($ major $)=8.2 \mathrm{~min} ; \mathrm{t}_{\mathrm{R} 2}($ minor $)=7.0 \mathrm{~min}$.

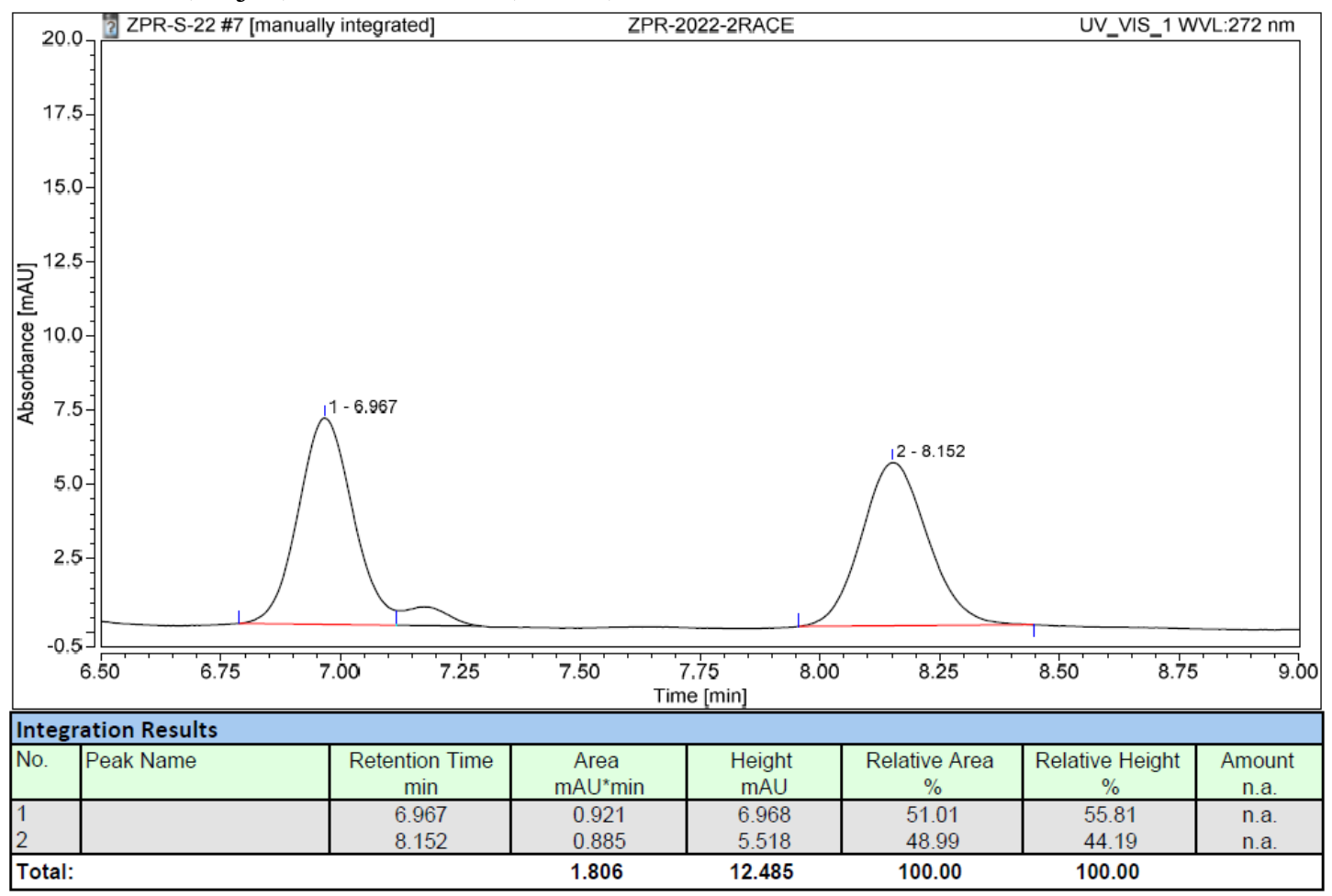




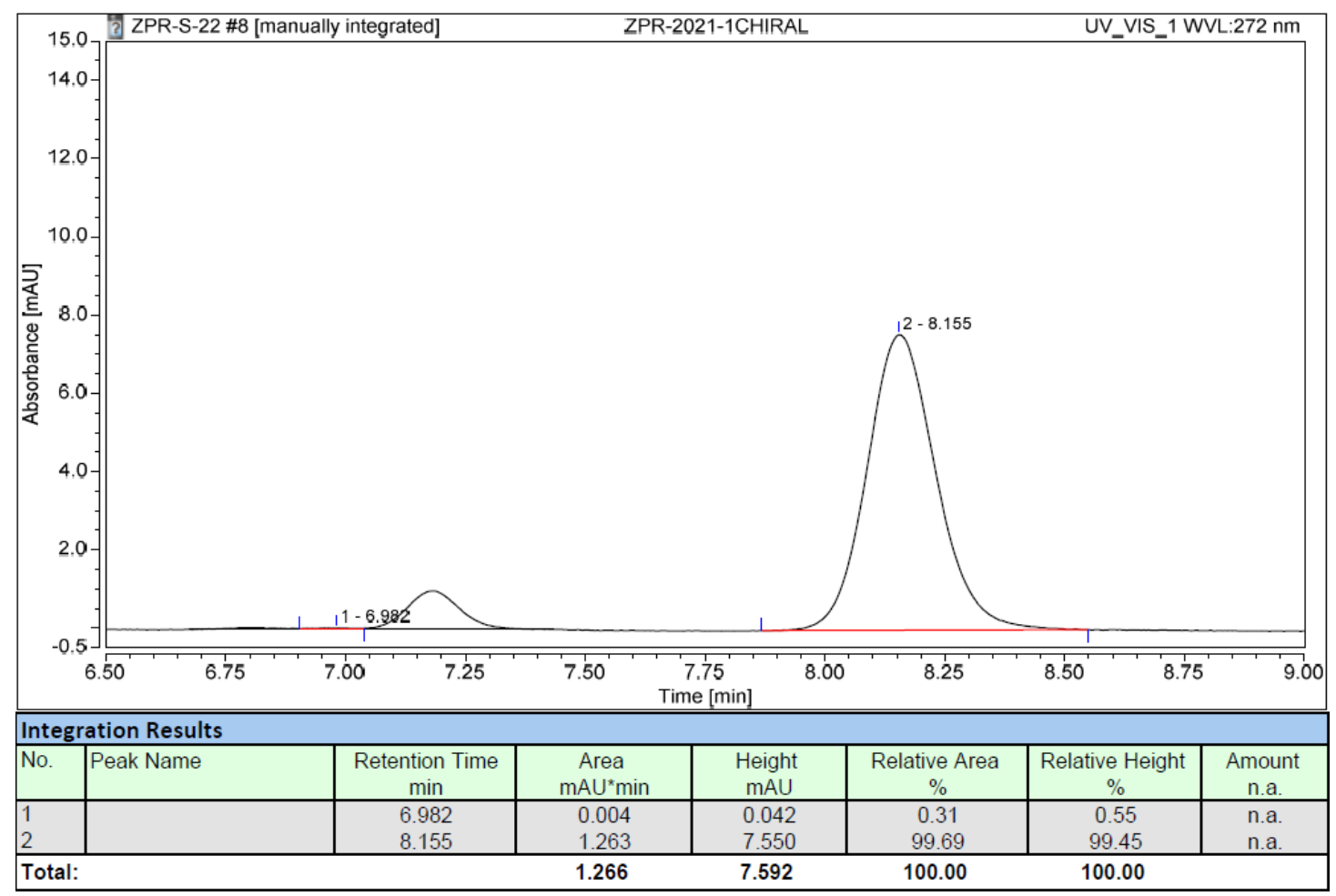<smiles>C=CC12Cc3ccccc3C(CP)C1(O)CCCC2=O</smiles>

$4 \mathbf{i}$

The title compound $\mathbf{4} \mathbf{i}$ was synthesized according to General Procedure (SI 4.1), and it was purified by column chromatography on silica gel (59\% yield, 98\% ee, $46.8 \mathrm{mg}$, white solid).

${ }^{1} \mathrm{H}$ NMR (400 MHz, Chloroform- $d$ ) $\delta 7.35$ (d, $\left.J=7.8 \mathrm{~Hz}, 1 \mathrm{H}\right), 7.21$ (dd, $J=7.8,7.6$ $\mathrm{Hz}, 1 \mathrm{H}), 7.15$ (dd, $J=7.6,7.5 \mathrm{~Hz}, 1 \mathrm{H}), 7.03$ (d, $J=7.5 \mathrm{~Hz}, 1 \mathrm{H}), 6.30-6.18(\mathrm{~m}, 1 \mathrm{H})$, 5.12-4.93 (m, 2H), 3.47-3.24 (m, 2H), 2.80-2.61 (m, 3H), 2.61-2.53 (m, 1H), 2.51-2.41 (m, 1H), 2.41-2.33 (m, 1H), 2.21-2.17 (m, 1H), 1.89-1.74 (m, 2H), 1.63-1.52 (m, 1H), $1.43(\mathrm{dd}, J=15.0,5.2 \mathrm{~Hz}, 2 \mathrm{H}), 1.28-1.24(\mathrm{~m}, 12 \mathrm{H}), 1.11(\mathrm{dd}, J=15.0,10.2 \mathrm{~Hz}, 2 \mathrm{H})$; ${ }^{13} \mathrm{C}$ NMR (101 MHz, Chloroform- $d$ ) $\delta 213.8,139.1,137.6,132.0,128.7,127.0,126.4$, 126.1, 118.1, 83.2, 80.3, 57.1, 43.2, 39.2, 36.4, 35.5, 25.0, 24.9, 24.9, 19.5; HRMS: m/z (ESI) calculated $\left[\mathrm{M}+\mathrm{NH}_{4}\right]^{+}: 414.2816$, found: $414.2813 .[\alpha]_{\mathrm{D}}{ }^{25}=+6.8\left(c=0.25, \mathrm{CHCl}_{3}\right)$. The enantiomeric excess of $\mathbf{4} \mathbf{i}$ was determined by chiral HPLC analysis after oxidation to the corresponding alcohol compared to the corresponding racemate.

Conditions: ChiralPak IF column; hexane $/ \mathrm{PrOH}=90: 10$; flow rate $=1.0 \mathrm{~mL} / \mathrm{min} ; \lambda=$ $272 \mathrm{~nm} ; \mathrm{t}_{\mathrm{R} 1}($ major $)=30.6 \mathrm{~min} ; \mathrm{t}_{\mathrm{R} 2}($ minor $)=13.0 \mathrm{~min}$. 

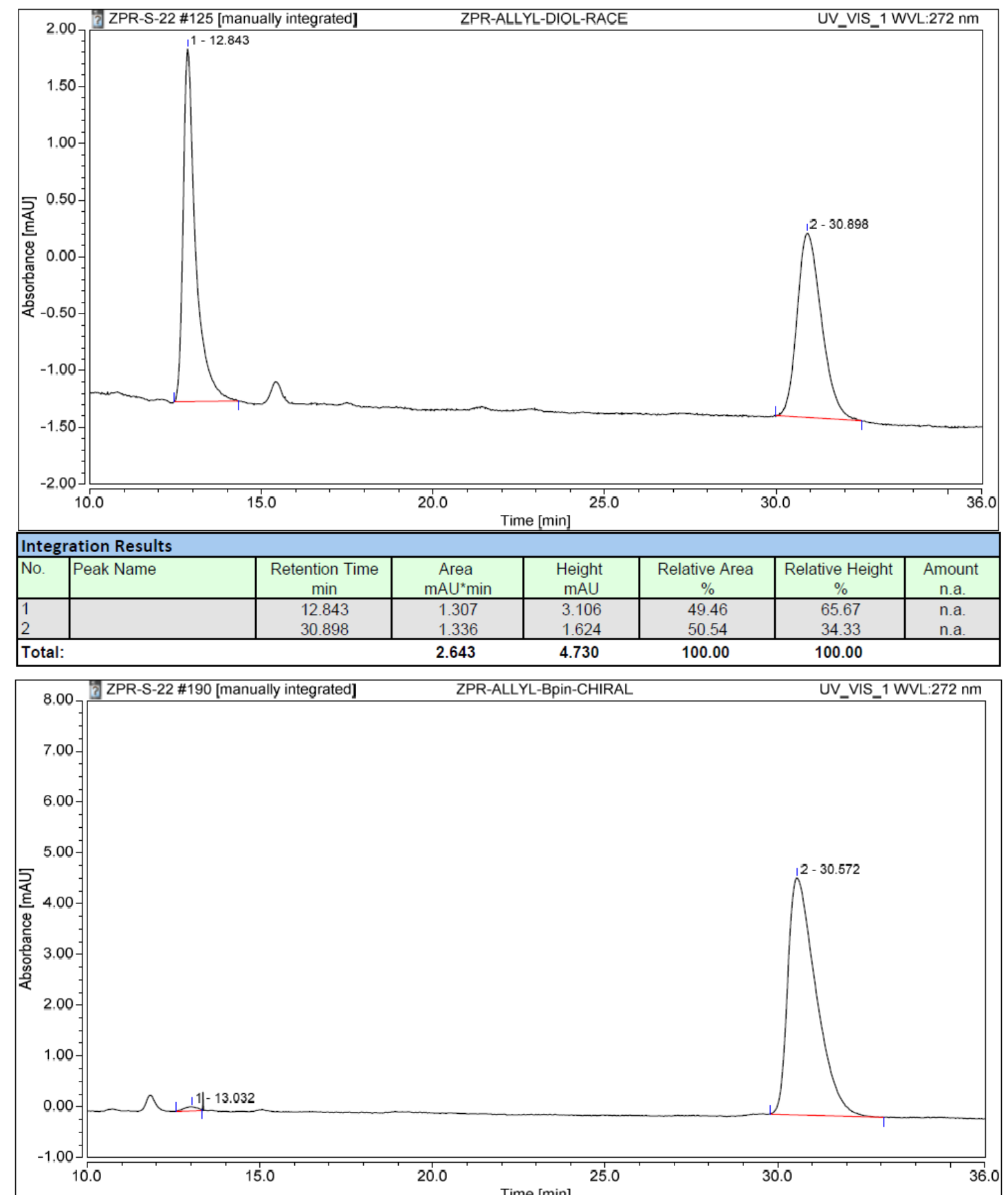

\begin{tabular}{|l|l|c|c|c|c|c|c|}
\hline \hline Integration Results \\
\hline No. & Peak Name & $\begin{array}{c}\text { Retention Time } \\
\text { min }\end{array}$ & $\begin{array}{c}\text { Area } \\
\text { mAU*min }\end{array}$ & $\begin{array}{c}\text { Height } \\
\mathrm{mAU}\end{array}$ & $\begin{array}{c}\text { Relative Area } \\
\%\end{array}$ & $\begin{array}{c}\text { Relative Height } \\
\%\end{array}$ & $\begin{array}{c}\text { Amount } \\
\text { n.a. }\end{array}$ \\
\hline 1 & & 13.032 & 0.037 & 0.086 & 0.84 & 1.80 & n.a. \\
2 & 30.572 & 4.404 & 4.671 & 99.16 & 98.20 & n.a. \\
\hline
\end{tabular}<smiles>CC(C)(C)OCCC[C@]12Cc3ccccc3C(C[Pb])[C@@]1(O)CCCC2=O</smiles> 
The title compound $\mathbf{4 j}$ was synthesized according to General Procedure (SI 4.1), and it was purified by column chromatography on silica gel (61\% yield, $98 \%$ ee, $59.1 \mathrm{mg}$, white solid).

${ }^{1} \mathrm{H}$ NMR (600 MHz, Chloroform- $d$ ) $\delta 7.31(\mathrm{~d}, J=7.9 \mathrm{~Hz}, 1 \mathrm{H}), 7.19(\mathrm{dd}, J=7.9,7.2$ $\mathrm{Hz}, 2 \mathrm{H}), 7.13(\mathrm{dd}, J=7.7,7.2 \mathrm{~Hz}, 1 \mathrm{H}), 7.04(\mathrm{~d}, J=7.7 \mathrm{~Hz}, 1 \mathrm{H}), 3.42(\mathrm{~d}, J=16.9 \mathrm{~Hz}$, $1 \mathrm{H}), 3.29$ (dd, $J=9.9,5.5 \mathrm{~Hz}, 2 \mathrm{H}), 2.77-2.65(\mathrm{~m}, 3 \mathrm{H}), 2.61-2.51(\mathrm{~m}, 1 \mathrm{H}), 2.35-2.29$ (m, 1H), 2.19-2.05 (m, 3H), 1.91-1.84 (m, 1H), 1.81-1.75 (m, 2H), 1.73-1.64 (m, 2H), $1.50(\mathrm{dd}, J=15.6,5.5 \mathrm{~Hz}, 1 \mathrm{H}), 1.39(\mathrm{~s}, 9 \mathrm{H}), 1.30-1.25(\mathrm{~m}, 12 \mathrm{H}), 1.12(\mathrm{dd}, J=15.6$, $9.9 \mathrm{~Hz}, 1 \mathrm{H}),{ }^{13} \mathrm{C}$ NMR $(151 \mathrm{MHz}$, Chloroform- $d$ ) $\delta 213.9,173.5,139.6,132.2,128.6$, 127.1, 126.6, 126.2, 83.7, 80.1, 80.0, 56.2, 43.2, 36.6, 36.4, 31.4, 28.1, 27.3, 24.9, 24.8, 23.3, 20.1;HRMS: m/z (ESI) calculated $[\mathrm{M}+\mathrm{H}]^{+}: 485.3074$, found: $485.3058 .[\alpha]_{\mathrm{D}}^{25}=$ $13.2\left(c=0.35, \mathrm{CHCl}_{3}\right)$.

The enantiomeric excess of $\mathbf{4} \mathbf{j}$ was determined by chiral HPLC analysis compared to the corresponding racemate.

Conditions: ChiralPak IG column; hexane $/{ }^{i} \mathrm{PrOH}=90: 10$; flow rate $=1.0 \mathrm{~mL} / \mathrm{min} ; \lambda=$ $272 \mathrm{~nm} ; \mathrm{t}_{\mathrm{R} 1}$ (major) $=14.4 \mathrm{~min} ; \mathrm{t}_{\mathrm{R} 2}$ (minor) $=9.6 \mathrm{~min}$.

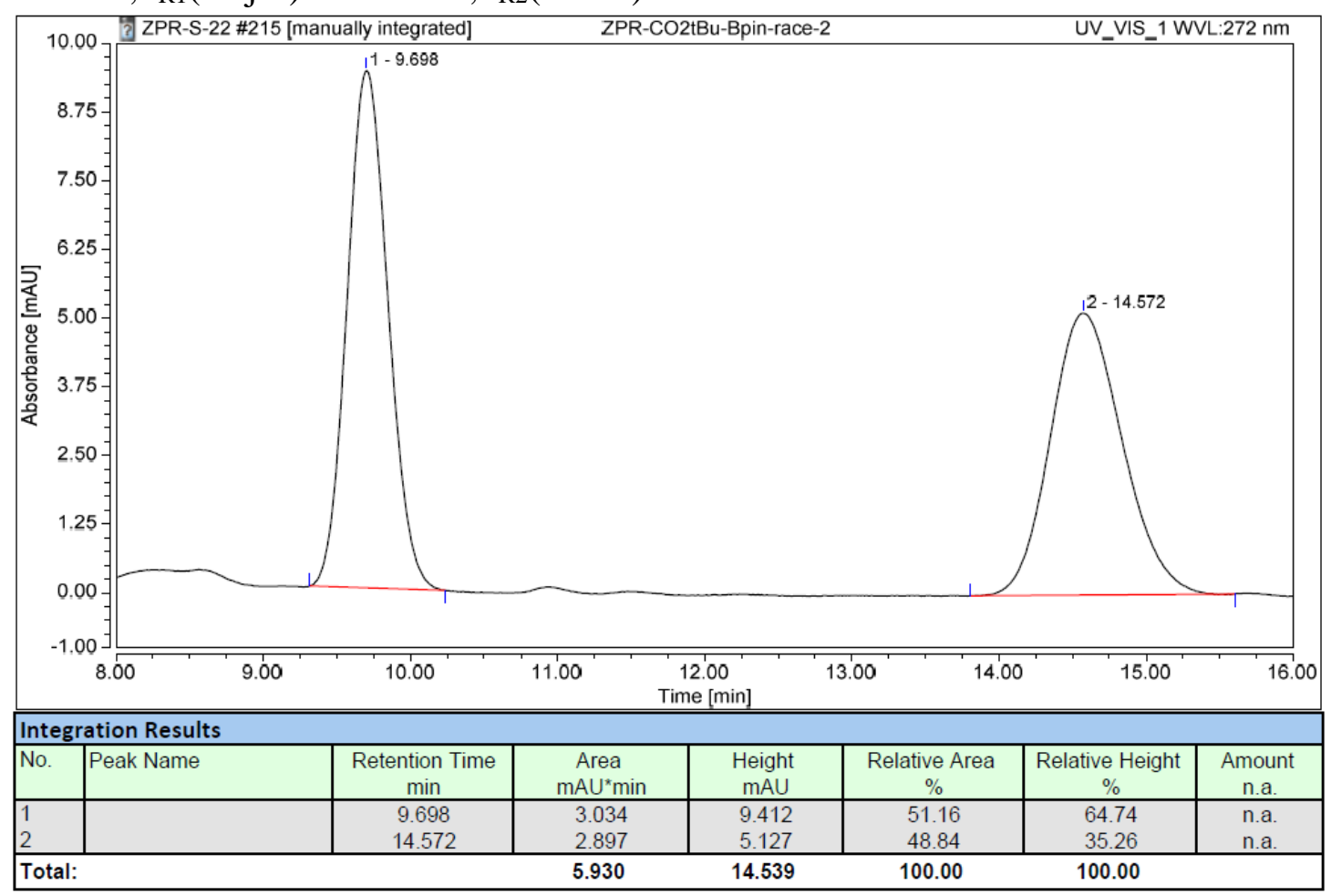




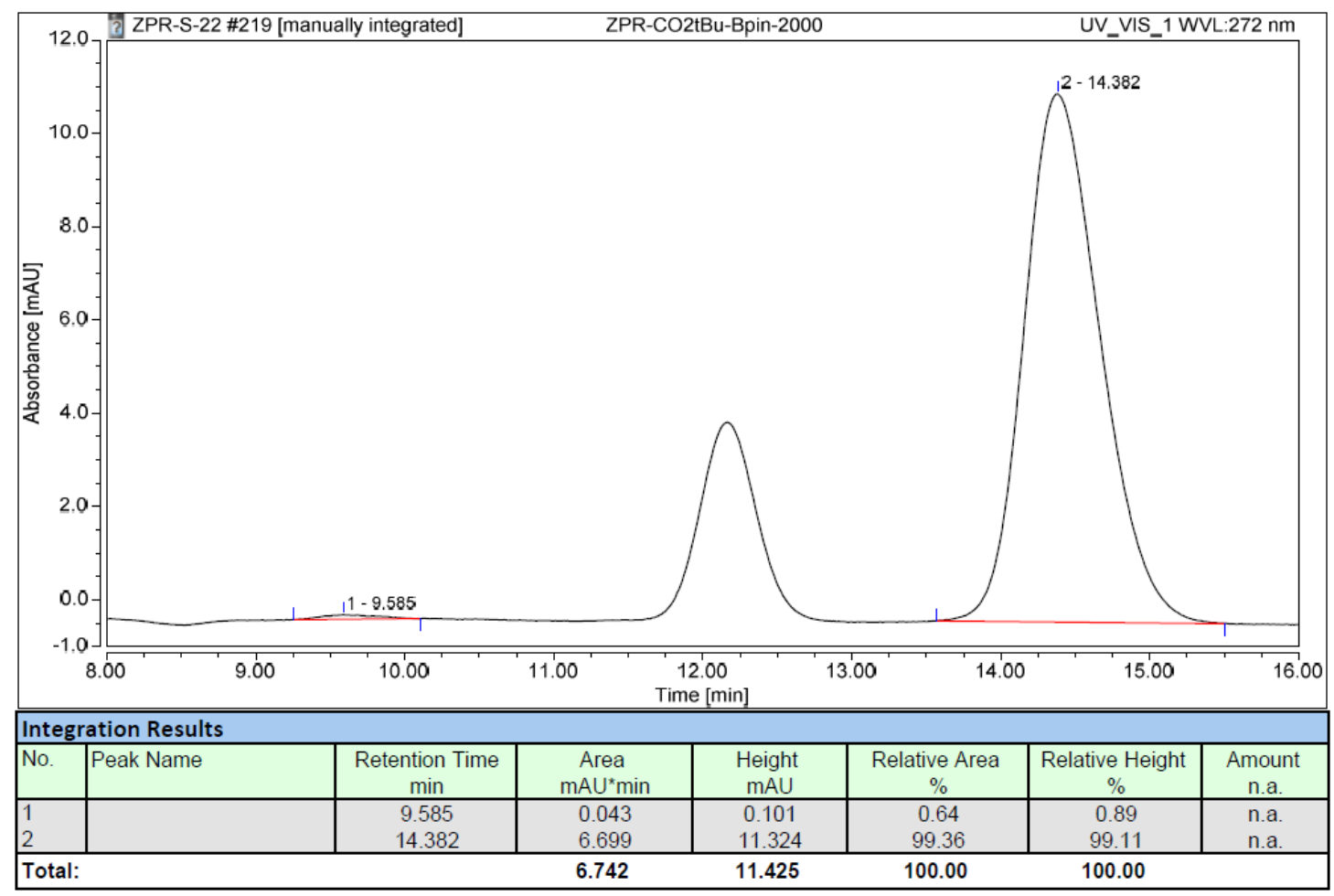<smiles>CC1(C)CC(=O)[C@]2(C)Cc3ccc(Cl)cc3[C@@H](C[Pb])[C@@]2(O)C1</smiles>

$4 \mathbf{k}$

The title compound $\mathbf{4 k}$ was synthesized according to General Procedure (SI 4.1), and it was purified by column chromatography on silica gel (62\% yield, $98 \%$ ee, $53.6 \mathrm{mg}$, white solid).

${ }^{1} \mathrm{H}$ NMR (400 MHz, Chloroform- $d$ ) $\delta 7.32(\mathrm{~s}, 1 \mathrm{H}), 7.12(\mathrm{~d}, J=8.0 \mathrm{~Hz}, 1 \mathrm{H}), 6.98(\mathrm{~d}, J$ $=8.0 \mathrm{~Hz}, 1 \mathrm{H}), 3.30(\mathrm{~d}, J=17.0 \mathrm{~Hz}, 1 \mathrm{H}), 3.00(\mathrm{dd}, J=10.0,5.2 \mathrm{~Hz}, 1 \mathrm{H}), 2.64(\mathrm{dd}, J=$ 29.6, $15.8 \mathrm{~Hz}, 2 \mathrm{H}), 2.44-2.14(\mathrm{~m}, 2 \mathrm{H}), 1.72(\mathrm{~d}, J=15.0 \mathrm{~Hz}, 1 \mathrm{H}), 1.53(\mathrm{~d}, J=15.0 \mathrm{~Hz}$, $1 \mathrm{H}), 1.44(\mathrm{dd}, J=15.2,5.2 \mathrm{~Hz}, 1 \mathrm{H}), 1.29(\mathrm{~s}, 12 \mathrm{H}), 1.16$ (s, 3H), $1.11(\mathrm{~s}, 3 \mathrm{H}), 1.14-1.08$ $(\mathrm{m}, 1 \mathrm{H}), 1.03(\mathrm{~s}, 3 \mathrm{H}) ;{ }^{13} \mathrm{C}$ NMR $(101 \mathrm{MHz}$, Chloroform- $d$ ) $\delta 214.1,141.2,132.3,130.9$, 130.0, 127.0, 126.3, 83.7, 79.2, 52.2, 49.4, 44.1, 39.7, 39.0, 34.3, 33.8, 30.8, 24.9, 24.8, 14.6; HRMS: $\mathrm{m} / \mathrm{z}$ (ESI) calculated $[\mathrm{M}+\mathrm{Na}]^{+}: 455.2136$, found: $455.2138 .[\alpha]_{\mathrm{D}}^{25}=+22.3$ $\left(c=0.08, \mathrm{CHCl}_{3}\right)$.

The enantiomeric excess of $\mathbf{4 k}$ was determined by chiral HPLC analysis compared to the corresponding racemate.

Conditions: ChiralPak IC column; hexane $/ \mathrm{PrOH}=90: 10$; flow rate $=0.5 \mathrm{~mL} / \mathrm{min} ; \lambda=$ $272 \mathrm{~nm} ; \mathrm{t}_{\mathrm{R} 1}($ major $)=12.4 \mathrm{~min} ; \mathrm{t}_{\mathrm{R} 2}($ minor $)=11.8 \mathrm{~min}$. 

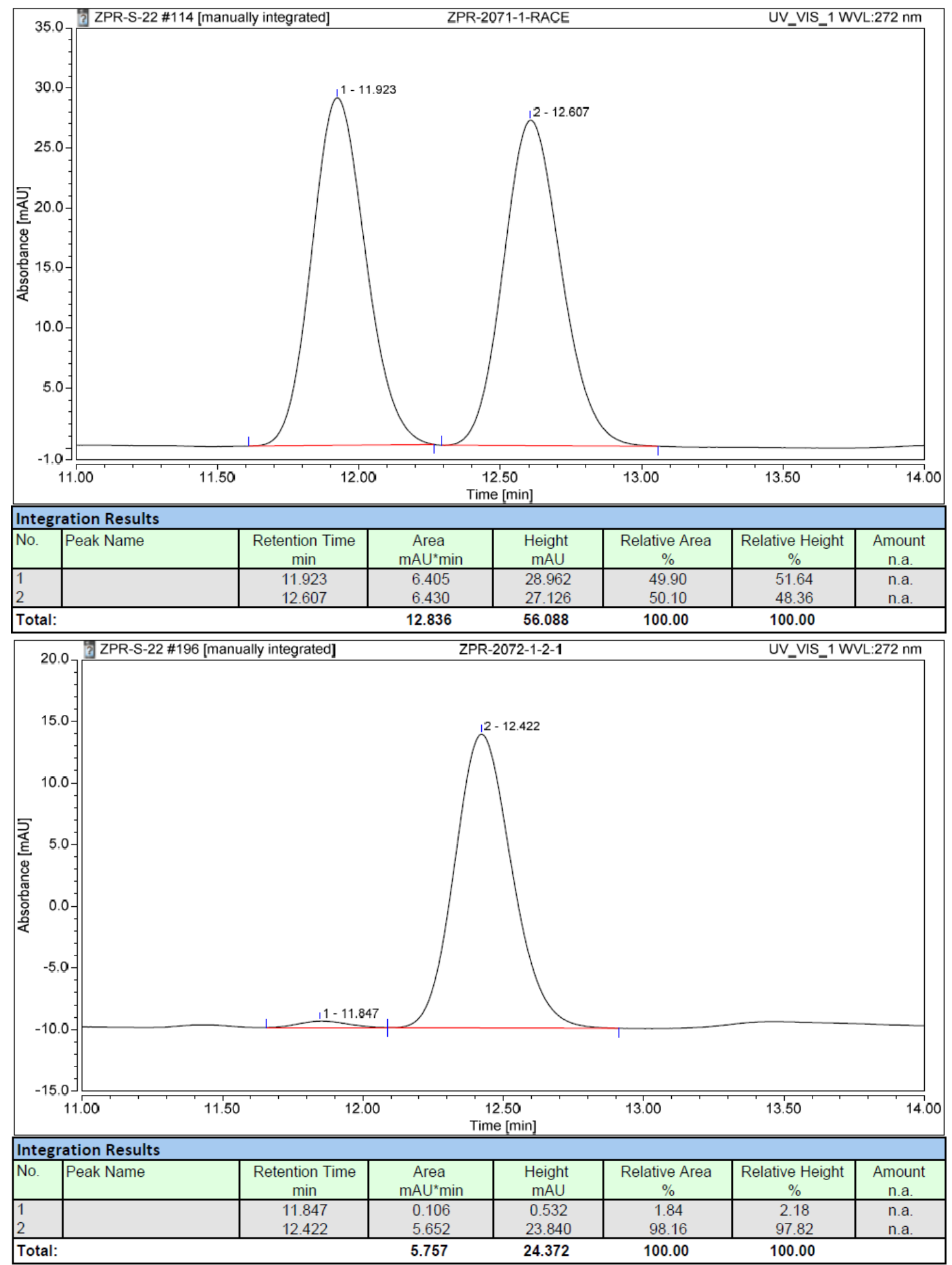<smiles>CC1(C/C=C/c2ccccc2)C(=O)CCC1=O</smiles>

The title compound 5a was synthesized according to General Procedure (SI 2.7), and it was purified by column chromatography on silica gel.

${ }^{1} \mathrm{H}$ NMR (400 MHz, Chloroform- $d$ ) $\delta$ 7.32-7.28 (m, 4H), 7.25-7.19 (m, 1H), 6.41 (d, $J$ $=15.6 \mathrm{~Hz}, 1 \mathrm{H}), 5.98(\mathrm{dt}, J=15.6,7.7 \mathrm{~Hz}, 1 \mathrm{H}), 2.85-2.62(\mathrm{~m}, 4 \mathrm{H}), 2.51$ (dd, $J=7.7$, 
$1.2 \mathrm{~Hz}, 2 \mathrm{H}), 1.17(\mathrm{~s}, 3 \mathrm{H}) ;{ }^{13} \mathrm{C}$ NMR (101 MHz, Chloroform- $d$ ) $\delta$ 216.3, 136.5, 134.7, 128.5, 127.6, 126.2, 122.6, 57.0, 39.2, 35.4, 19.0; HRMS: m/z (ESI) calculated $[\mathrm{M}+\mathrm{H}]^{+}: 229.1229$, found:229.1232.<smiles>CC1(C/C=C/c2ccccc2)C(=O)CCCC1=O</smiles>

The title compound $\mathbf{5 b}$ was synthesized according to General Procedure (SI 2.7), and it was purified by column chromatography on silica gel.

${ }^{1} \mathrm{H}$ NMR (400 MHz, Chloroform- $d$ ) $\delta$ 7.33-7.24 (m, 4H), 7.23-7.18 (m, 1H), 6.41 (d, $J$ $=15.8 \mathrm{~Hz}, 1 \mathrm{H}), 5.97(\mathrm{dt}, J=15.8,7.5 \mathrm{~Hz}, 1 \mathrm{H}), 2.76-2.50(\mathrm{~m}, 6 \mathrm{H}), 2.07-1.81(\mathrm{~m}, 2 \mathrm{H})$, $1.30(\mathrm{~s}, 3 \mathrm{H}) ;{ }^{13} \mathrm{C}$ NMR (101 MHz, Chloroform- $d$ ) $\delta$ 210.0, 136.8, 134.1, 128.5, 127.5, 126.2, 123.7, 65.3, 40.3, 38.3, 20.3, 17.4; HRMS: $\mathrm{m} / \mathrm{z}$ (ESI) calculated $[\mathrm{M}+\mathrm{H}]^{+}: 243.1385$, found:243.1382.

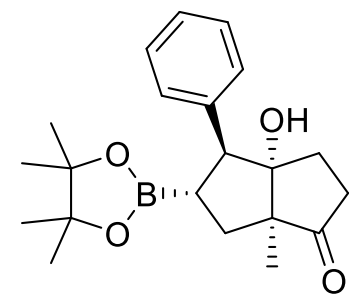

$6 \mathbf{a}$

The title compound 6a was synthesized according to General Procedure (SI 4.1), and it was purified by column chromatography on silica gel (90\% yield, $61 \% e e, 64.1 \mathrm{mg}$, white solid).

${ }^{1} \mathrm{H}$ NMR (600 MHz, Chloroform- $d$ ) $\delta$ 7.37-7.29 (m, 1H), $7.26(\mathrm{td}, J=6.0,5.0,2.7 \mathrm{~Hz}$, $0 \mathrm{H}), 3.32(\mathrm{~d}, J=11.8 \mathrm{~Hz}, 1 \mathrm{H}), 2.43(\mathrm{~m}, 1 \mathrm{H}), 2.22-2.12(\mathrm{~m}, 2 \mathrm{H}), 1.92-1.84(\mathrm{~m}, 1 \mathrm{H})$, $1.82(\mathrm{~s}, 1 \mathrm{H}), 1.72-1.57(\mathrm{~m}, 2 \mathrm{H}), 1.44-1.36(\mathrm{~m}, 1 \mathrm{H}), 1.17(\mathrm{~s}, 3 \mathrm{H}), 1.09(\mathrm{~s}, 12 \mathrm{H}) ;{ }^{13} \mathrm{C}$ NMR (101 MHz, Chloroform- $d$ ) $\delta$ 222.2, 138.5, 128.2, 128.1, 126.8, 89.7, 83.4, 58.5, 57.8, 37.1, 36.4, 30.2, 24.5, 24.4, 18.4; HRMS: $\mathrm{m} / \mathrm{z}$ (ESI) calculated $[\mathrm{M}+\mathrm{Na}]^{+}: 379.2057$, found: $379.2062 ;[\alpha]_{\mathrm{D}}{ }^{25}=-39.5\left(c=0.14, \mathrm{CHCl}_{3}\right)$.

The enantiomeric excess of 6a was determined by chiral HPLC analysis compared to the corresponding racemate. The configuration of $\mathbf{6 a}$ was determined by 2D NOESY analysis and 2D COSY analysis

Conditions: ChiralPak IA column; hexane $/{ }^{i} \mathrm{PrOH}=95: 5$; flow rate $=0.8 \mathrm{~mL} / \mathrm{min} ; \lambda=$ $220 \mathrm{~nm} ; \mathrm{t}_{\mathrm{R} 1}$ (major) $=18.1 \mathrm{~min} ; \mathrm{t}_{\mathrm{R} 2}($ minor $)=19.2 \mathrm{~min}$. 


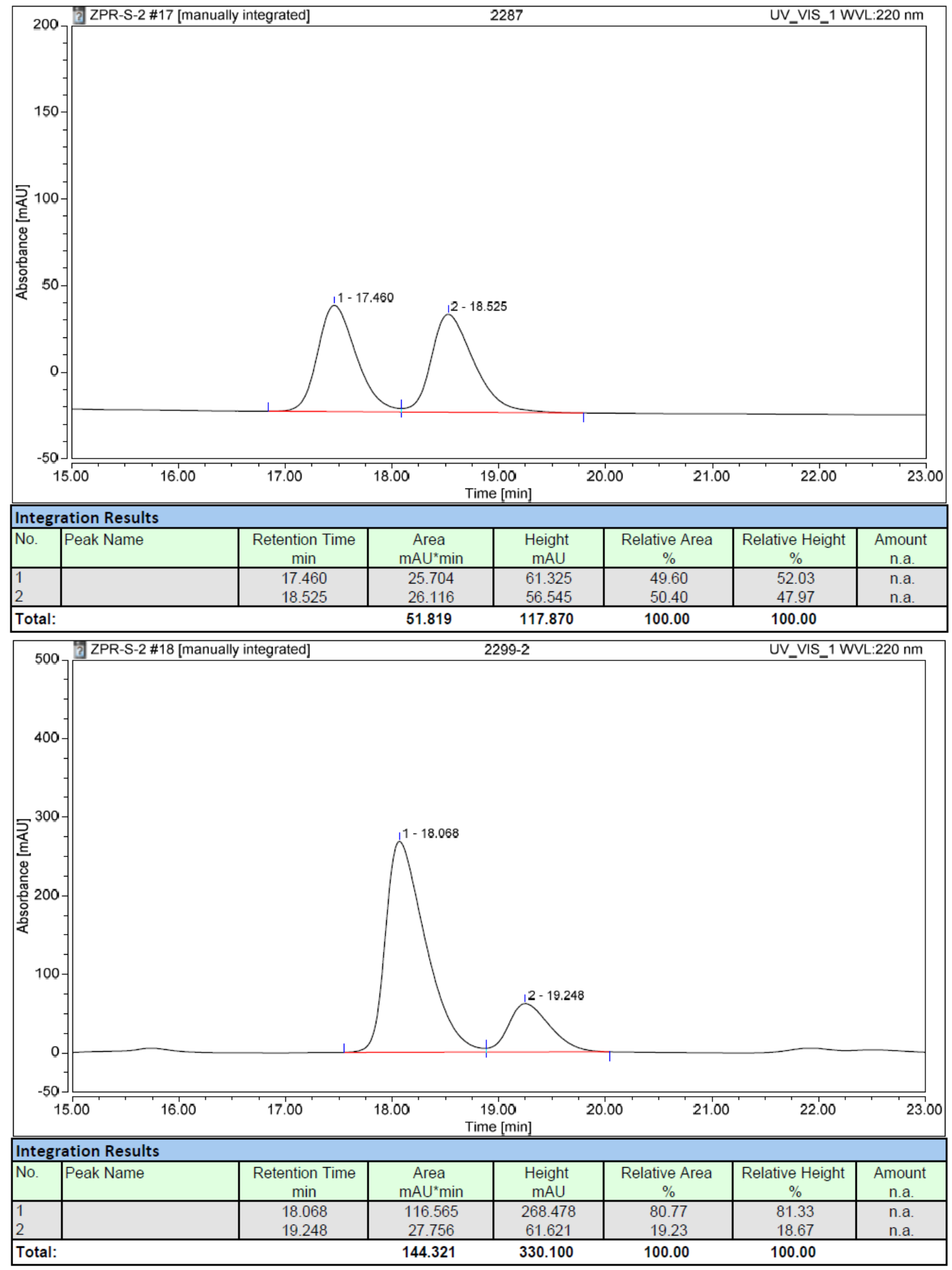


2D COSY analysis of $\mathbf{6 a}$
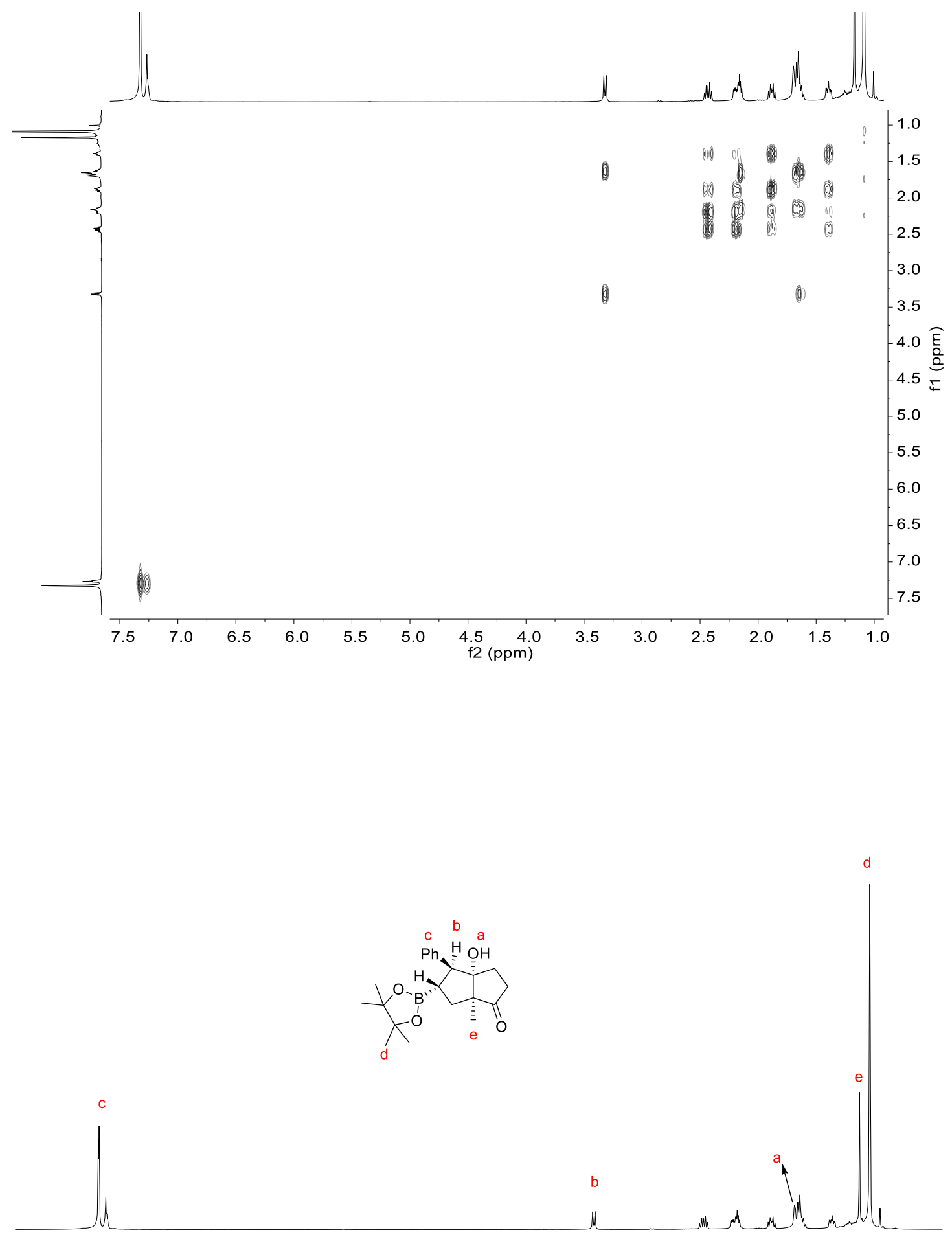

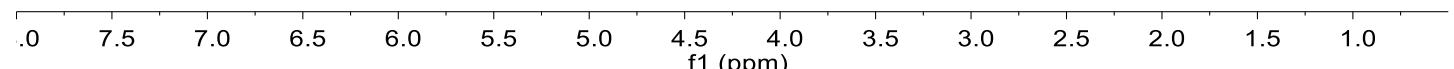



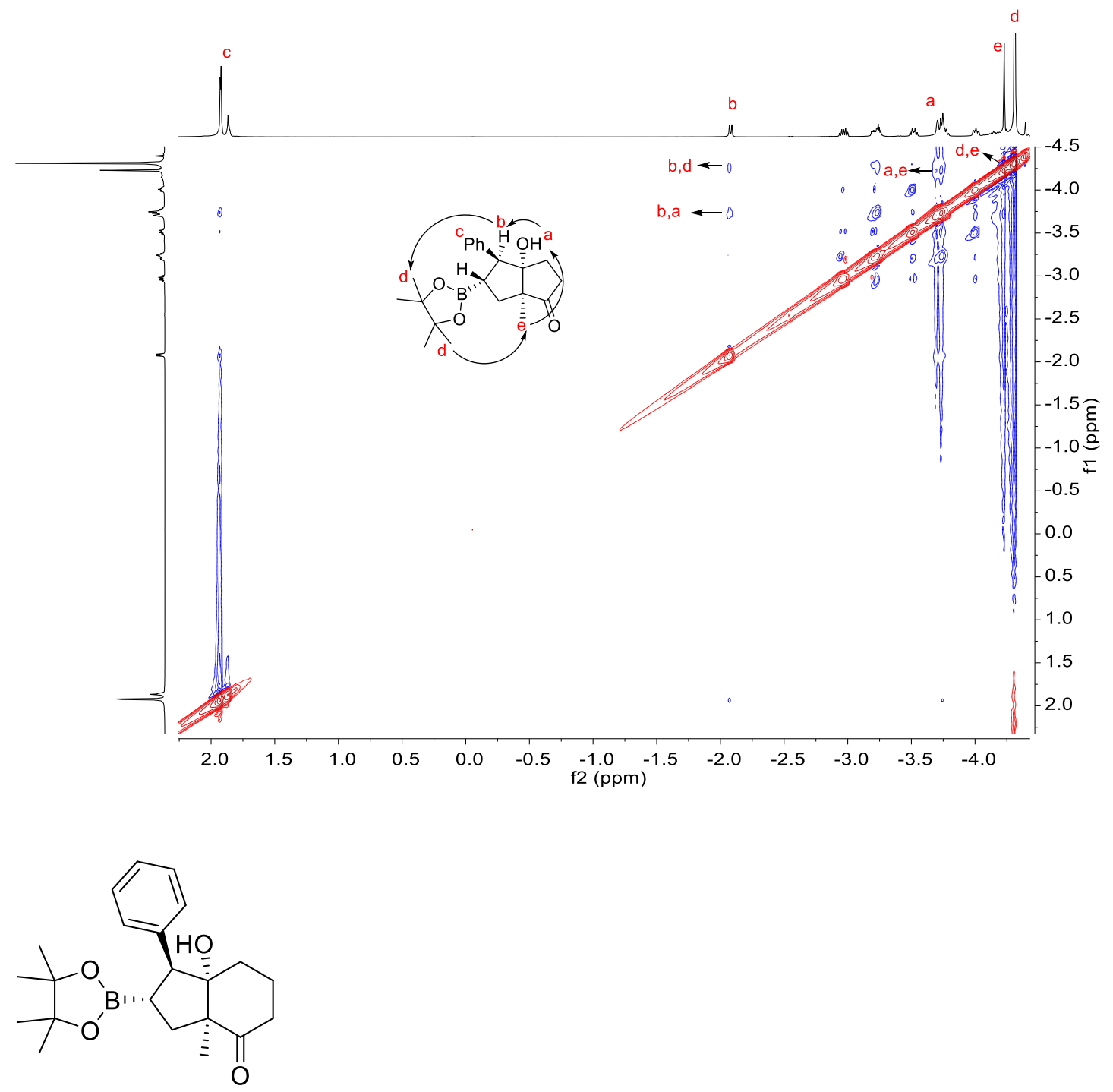

6b

The title compound $\mathbf{6 b}$ was synthesized according to General Procedure (SI 4.3), and it was purified by column chromatography on silica gel (58\% yield, 62\% ee, $42.9 \mathrm{mg}$, white solid).

${ }^{1} \mathrm{H}$ NMR (400 MHz, Chloroform- $d$ ) $\delta$ 7.35-7.28 (m, 4H), 7.27-7.19 (m, 1H), 3.46 (d, $J$ $=11.9 \mathrm{~Hz}, 1 \mathrm{H}), 2.53-2.43(\mathrm{~m}, 1 \mathrm{H}), 2.42-2.32(\mathrm{~m}, 2 \mathrm{H}), 2.04-1.95(\mathrm{~m}, 1 \mathrm{H}), 1.90-1.80(\mathrm{~m}$, $1 \mathrm{H}), 1.78-1.66(\mathrm{~m}, 2 \mathrm{H}), 1.64-1.56(\mathrm{~m}, 3 \mathrm{H}), 1.33(\mathrm{~s}, 3 \mathrm{H}), 1.12(\mathrm{~s}, 12 \mathrm{H}),{ }^{13} \mathrm{C}$ NMR $(101$ $\mathrm{MHz}$, Chloroform- $d$ ) $\delta$ 214.4, 138.6, 128.5, 128.1, 126.7, 86.6, 83.4, 58.4, 56.2, 37.1, 35.9, 30.8, 24.6, 24.5, 20.0, 18.5; HRMS: $\mathrm{m} / \mathrm{z}(\mathrm{ESI})$ calculated $[\mathrm{M}+\mathrm{Na}]^{+}:$3393.2213, found: $3393.2209 .[\alpha]_{\mathrm{D}}{ }^{25}=-25.2\left(c=0.10, \mathrm{CHCl}_{3}\right)$.

The enantiomeric excess of $\mathbf{6} \mathbf{b}$ was determined by chiral HPLC analysis compared to the corresponding racemate. The configuration of $\mathbf{6 b}$ was determined by 2D NOESY analysis 
Conditions: Conditions: ChiralPak IG column; hexane $/{ }^{i} \mathrm{PrOH}=90: 10$; flow rate $=1.0$ $\mathrm{mL} / \mathrm{min} ; \lambda=220 \mathrm{~nm} ; \mathrm{t}_{\mathrm{R} 1}$ (major) $=14.0 \mathrm{~min} ; \mathrm{t}_{\mathrm{R} 2}($ minor $)=12.1 \mathrm{~min}$.
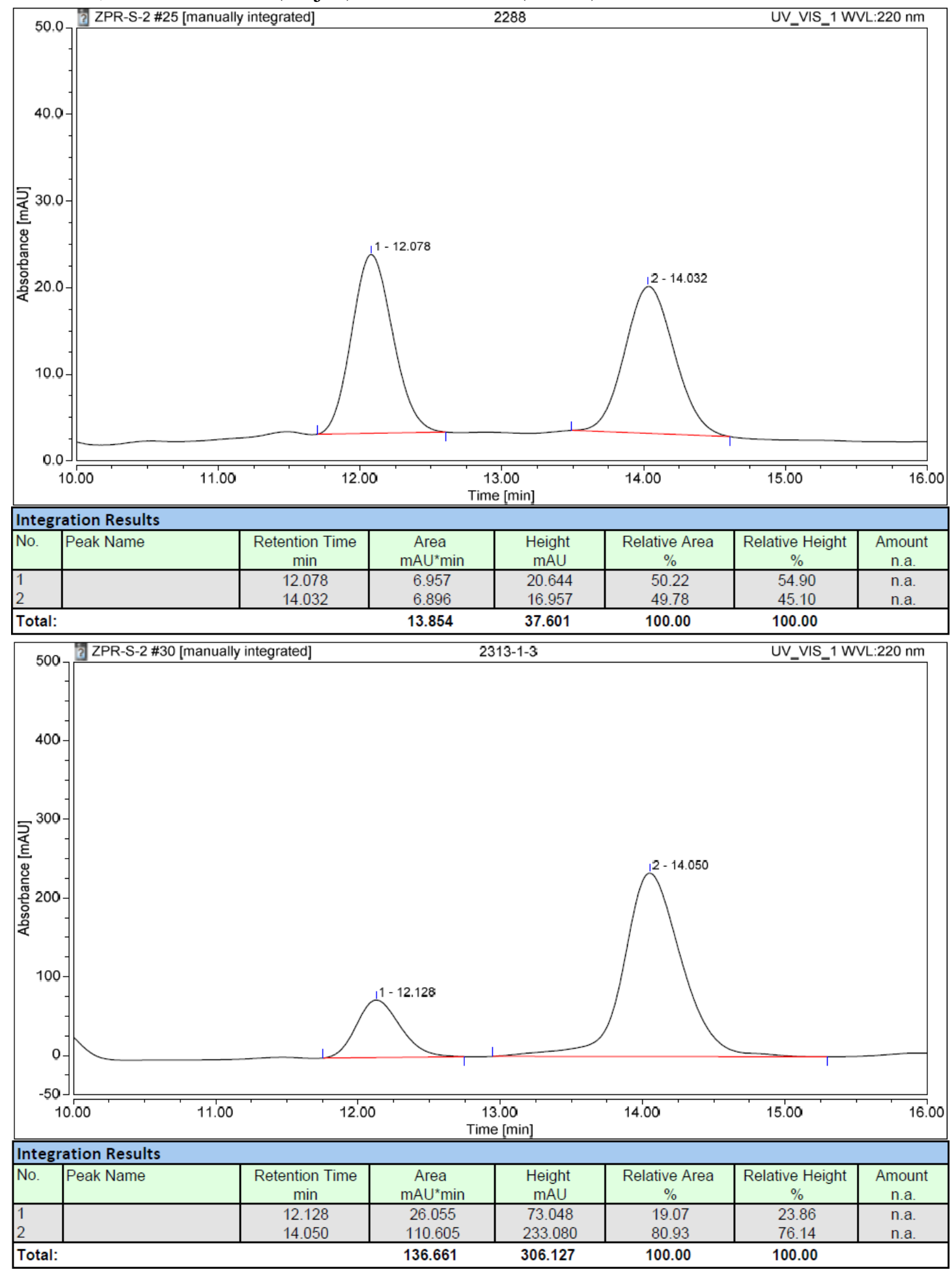
2D NOESY analysis of $\mathbf{6 b}$

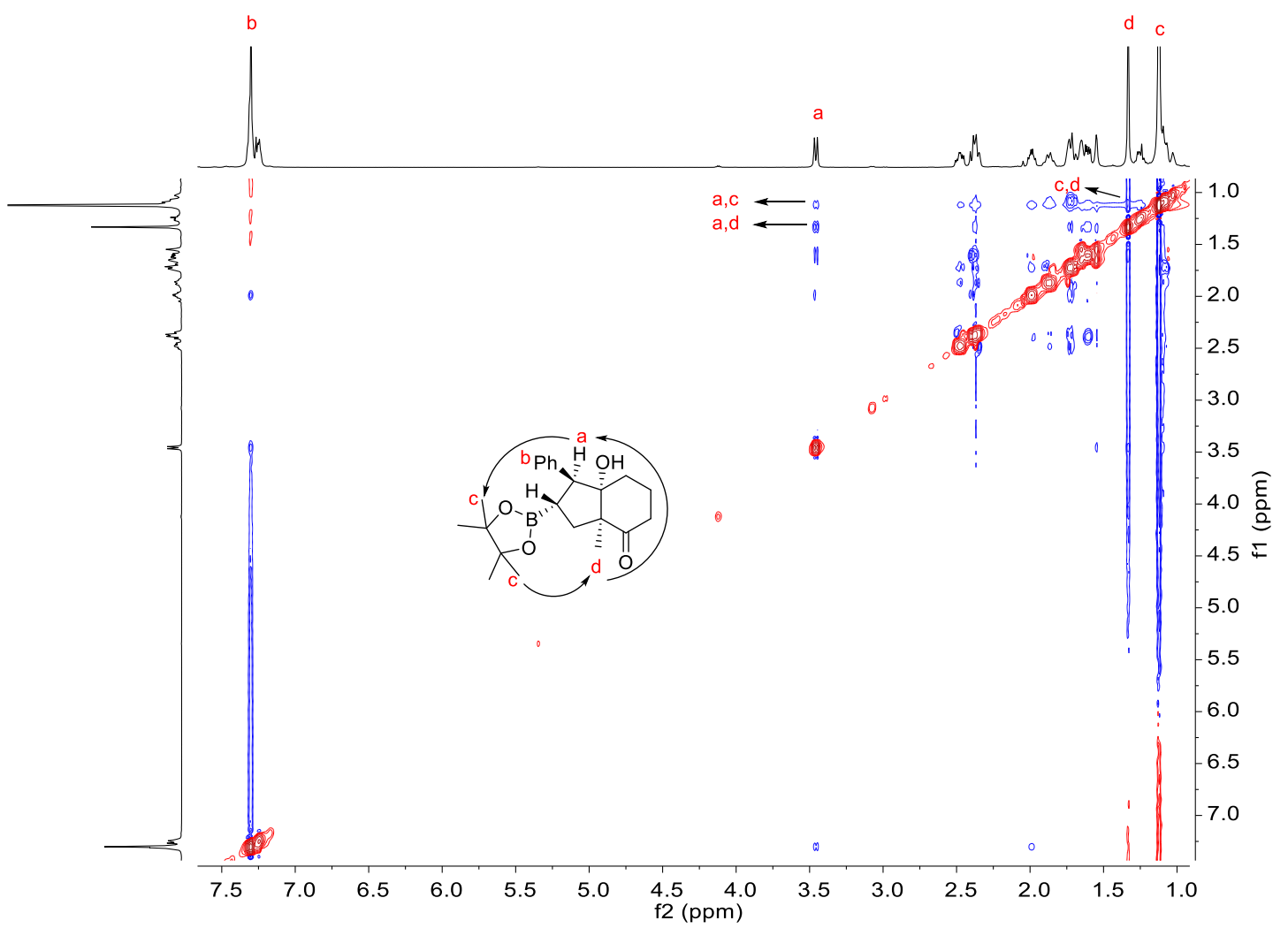<smiles>O=C1CCC[C@@]2(O)[C@@H]1Cc1ccccc1[C@@H]2CO</smiles>

The title compound 7a was synthesized according to General Procedure (SI 6.1), and it was purified by column chromatography on silica gel ( $84 \%$ yield, $98 \%$ ee, $43.7 \mathrm{mg}$, white solid).

${ }^{1} \mathrm{H}$ NMR (600 MHz, Chloroform- $d$ ) $\delta$ 7.26-7.22 (m, 1H), 7.20-7.17 (m, 2H), 7.11-7.07 $(\mathrm{m}, 1 \mathrm{H}), 4.61(\mathrm{dd}, J=10.6,5.4 \mathrm{~Hz}, 1 \mathrm{H}), 4.13(\mathrm{dd}, J=10.6,10.6 \mathrm{~Hz}, 1 \mathrm{H}), 3.51(\mathrm{~s}, 1 \mathrm{H})$, $3.46(\mathrm{~d}, J=16.9 \mathrm{~Hz}, 1 \mathrm{H}), 3.31(\mathrm{dd}, J=10.6,5.4 \mathrm{~Hz}, 1 \mathrm{H}), 2.84-2.70(\mathrm{~m}, 1 \mathrm{H}), 2.64-2.51$ (m, 2H), 2.44-2.36 (m, 1H), 2.30-2.18 (m, 1H), 2.03-1.93 (m, 1H), 1.92-1.85 (m, 1H), 1.17 (s, 3H); ${ }^{13} \mathrm{C}$ NMR (101 MHz, Chloroform-d) $\delta$ 214.2, 134.1, 132.8, 129.6, 126.7, 126.6, 126.0, 78.7, 63.5, 53.3, 45.5, 39.7, 36.0, 26.4, 20.0, 14.0; HRMS: m/z (ESI) calculated $\left[\mathrm{M}+\mathrm{NH}_{4}\right]^{+}: 278.1756$, found: $278.1753 .[\alpha]_{\mathrm{D}}{ }^{25}=+83.8\left(c=0.21, \mathrm{CHCl}_{3}\right)$.

The enantiomeric excess of 7a was determined by chiral HPLC analysis compared to the corresponding racemate.

Conditions: ChiralPak IA column; hexane $/{ }^{i} \mathrm{PrOH}=90: 10$; flow rate $=0.8 \mathrm{~mL} / \mathrm{min} ; \lambda=$ $272 \mathrm{~nm} ; \mathrm{t}_{\mathrm{R} 1}($ major $)=28.2 \mathrm{~min} ; \mathrm{t}_{\mathrm{R} 2}($ minor $)=16.7 \mathrm{~min}$. 

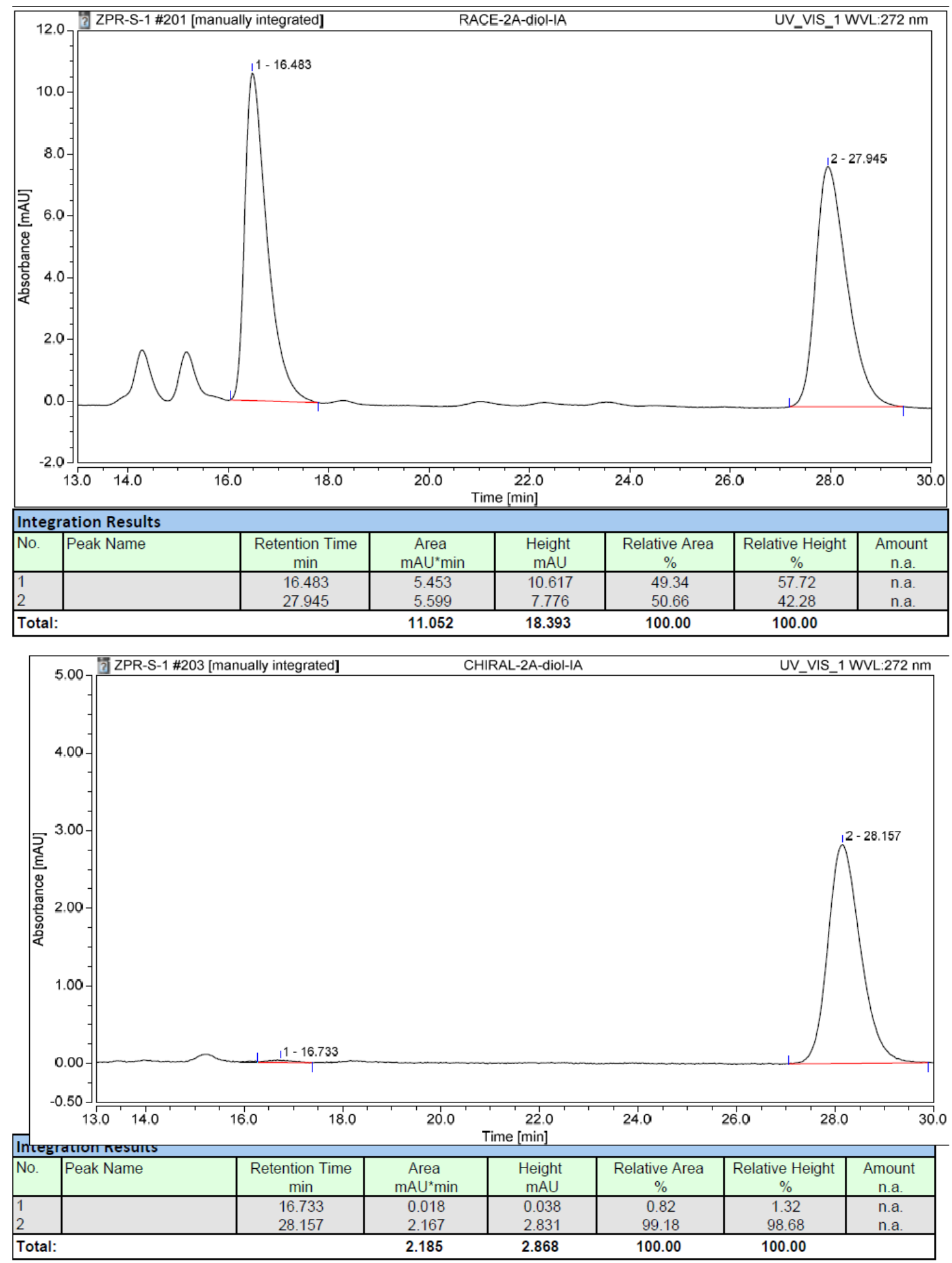
<smiles>O=C1CCC[C@@]2(O)[C@H](CC(F)(F)F)c3ccccc3C[C@]12O</smiles>

The title compound $\mathbf{7 b}$ was synthesized according to General Procedure (SI 6.2), and it was purified by column chromatography on silica gel (76\% yield, $52.3 \mathrm{mg}$, white solid).

${ }^{1} \mathrm{H}$ NMR $\left(600 \mathrm{MHz}\right.$, Methanol- $\left.d_{4}\right) \delta 7.62(\mathrm{~d}, J=7.9 \mathrm{~Hz}, 1 \mathrm{H}), 7.10(\mathrm{dd}, J=7.9,7.5 \mathrm{~Hz}$, $1 \mathrm{H}), 7.04(\mathrm{dd}, J=7.5,7.5 \mathrm{~Hz}, 1 \mathrm{H}), 6.97(\mathrm{~d}, J=7.5 \mathrm{~Hz}, 1 \mathrm{H}), 3.48(\mathrm{~d}, J=16.8 \mathrm{~Hz}, 1 \mathrm{H})$, $3.31(\mathrm{~s}, 1 \mathrm{H}), 3.05-2.97(\mathrm{~m}, 1 \mathrm{H}), 2.87-2.78(\mathrm{~m}, 1 \mathrm{H}), 2.45(\mathrm{~d}, J=16.8 \mathrm{~Hz}, 1 \mathrm{H}), 2.24-2.16$ (m, 1H), 2.14-2.01 (m, 1H), 1.98-1.91 (m, 1H), 1.90-1.77 (m, 1H), 1.68-1.59 (m, 1H), $1.08(\mathrm{~s}, 3 \mathrm{H}), 0.94-0.79(\mathrm{~m}, 1 \mathrm{H}), 0.74-0.65(\mathrm{~m}, 1 \mathrm{H}) ;{ }^{13} \mathrm{C}$ NMR $\left(151 \mathrm{MHz}\right.$, Methanol- $\left.d_{4}\right)$ $\delta 216.8,141.2,131.1,127.5,127.3,124.9,124.2$, 78.5, 53.1, 41.1, 39.3, 35.0, 28.7, 24.7, 19.1, 12.9; ${ }^{19} \mathrm{~F}$ NMR $\left(376 \mathrm{MHz}\right.$, Methanol- $\left.d_{4}\right) \delta-139.0 ;$ HRMS: m/z (ESI) calculated $[\mathrm{M}-\mathrm{K}]^{-}: 311.1430$, found: $311.1443 .[\alpha]_{\mathrm{D}}^{25}=+40.4(c=0.28$, Acetone $)$.<smiles>O=C1CCC[C@@]2(O)[C@@H]1Cc1ccccc1[C@H]2Cc1ccccc1</smiles>

The title compound 7c was synthesized according to General Procedure (SI 6.3), and it was purified by column chromatography on silica gel (95\% yield, 99\% ee, $52.3 \mathrm{mg}$, white solid).

${ }^{1} \mathrm{H}$ NMR (400 MHz, Chloroform- $d$ ) $\delta$ 7.41-7.30 (m, 5H), 7.26-7.21 (m, 1H), 7.18-7.05 $(\mathrm{m}, 3 \mathrm{H}), 3.50(\mathrm{~d}, J=17.2 \mathrm{~Hz}, 1 \mathrm{H}), 3.45-3.30(\mathrm{~m}, 2 \mathrm{H}), 3.11(\mathrm{dd}, J=15.0,7.9 \mathrm{~Hz}, 1 \mathrm{H})$, 2.88-2.72 (m, 1H), $2.65(\mathrm{~d}, J=17.2 \mathrm{~Hz}, 1 \mathrm{H}), 2.42-2.34(\mathrm{~m}, 1 \mathrm{H}), 2.16-2.03(\mathrm{~m}, 1 \mathrm{H})$, 1.99-1.84 (m, 3H), $1.59(\mathrm{~s}, 1 \mathrm{H}), 1.21(\mathrm{~s}, 3 \mathrm{H}) ;{ }^{13} \mathrm{C}$ NMR (101 MHz, Chloroform- $d$ ) $\delta$ 213.9, 142.0, 138.3, 132.2, 129.0, 128.9, 128.5, 127.4, 126.4, 126.4, 126.3, 78.9, 53.6, 46.4, 39.8, 35.8, 35.1, 26.4, 19.8, 14.5; HRMS: $\mathrm{m} / \mathrm{z}(\mathrm{ESI})$ calculated $[\mathrm{M}+\mathrm{K}]^{+}: 359.1413$, found: $359.1414 .[\alpha]_{\mathrm{D}}{ }^{25}=+65.5\left(c=0.22, \mathrm{CHCl}_{3}\right)$.

The enantiomeric excess of 7c was determined by chiral HPLC analysis compared to the corresponding racemate.

Conditions: ChiralPak IC column; hexane $/{ }^{i} \mathrm{PrOH}=95: 5$; flow rate $=1.0 \mathrm{~mL} / \mathrm{min} ; \lambda=$ $272 \mathrm{~nm} ; \mathrm{t}_{\mathrm{R} 1}($ major $)=25.0 \mathrm{~min} ; \mathrm{t}_{\mathrm{R} 2}($ minor $)=37.4 \mathrm{~min}$. 

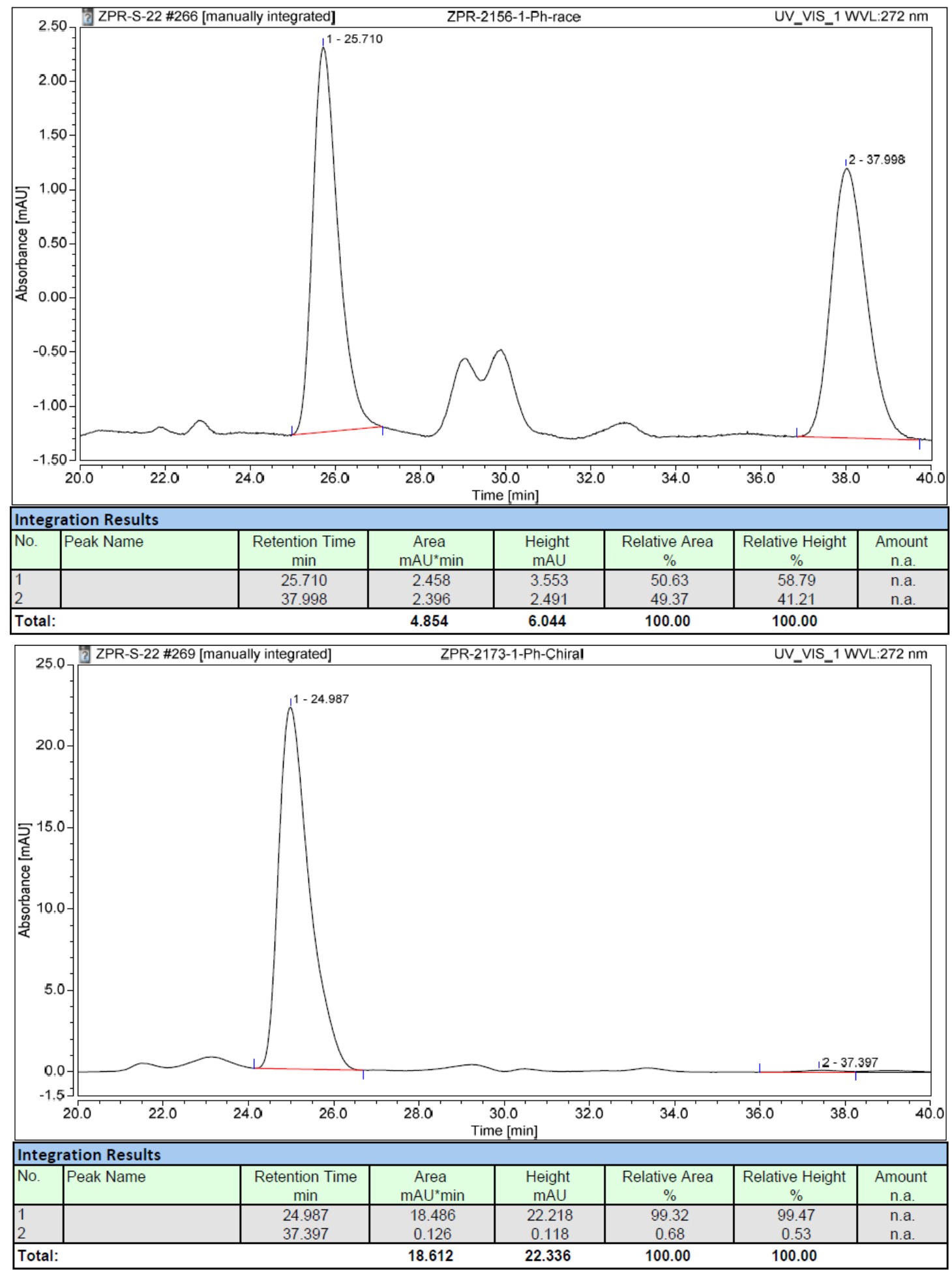
<smiles>CC(C)=CC[C@H]1c2ccccc2C[C@]2(O)C(=O)CCC[C@]12O</smiles>

The title compound 7d was synthesized according to General Procedure (SI 6.4), and it was purified by column chromatography on silica gel (91\% yield, 97\% ee, $52.3 \mathrm{mg}$, white solid).

${ }^{1} \mathrm{H}$ NMR (600 MHz, Chloroform- $d$ ) $\delta 7.42(\mathrm{~d}, J=7.6 \mathrm{~Hz}, 1 \mathrm{H}), 7.22-7.14(\mathrm{~m}, 2 \mathrm{H}), 7.06$ $(\mathrm{d}, J=7.3 \mathrm{~Hz}, 1 \mathrm{H}), 5.40(\mathrm{t}, J=6.8 \mathrm{~Hz}, 1 \mathrm{H}), 3.45(\mathrm{~d}, J=16.9 \mathrm{~Hz}, 1 \mathrm{H}), 3.00(\mathrm{t}, J=6.4$ $\mathrm{Hz}, 1 \mathrm{H}), 2.80-2.71(\mathrm{~m}, 1 \mathrm{H}), 2.69-2.57(\mathrm{~m}, 3 \mathrm{H}), 2.40-2.32(\mathrm{~m}, 1 \mathrm{H}), 2.11(\mathrm{dm}, 1 \mathrm{H}), 1.95-$ $1.88(\mathrm{~m}, 1 \mathrm{H}), 1.86-1.67(\mathrm{~m}, 9 \mathrm{H}), 1.16(\mathrm{~s}, 3 \mathrm{H}) ;{ }^{13} \mathrm{C} \mathrm{NMR}(151 \mathrm{MHz}$, Chloroform- $d$ ) $\delta$ 214.2, 138.1, 133.1, 132.3, 129.0, 127.1, 126.4, 126.2, 124.8, 79.0, 53.5, 45.9, 39.7, 35.9, 27.8, 26.6, 25.8, 19.9, 18.3, 14.4; HRMS: $\mathrm{m} / \mathrm{z}$ (ESI) calculated $[\mathrm{M}+\mathrm{Na}]^{+}: 321.1831$, found: $321.1838 .[\alpha]_{\mathrm{D}}{ }^{25}=+63.1\left(c=0.10, \mathrm{CHCl}_{3}\right)$.

The enantiomeric excess of $\mathbf{7 d}$ was determined by chiral HPLC analysis compared to the corresponding racemate.

Conditions: ChiralPak IC column; hexane $/{ }^{i} \mathrm{PrOH}=80: 20$; flow rate $=1.0 \mathrm{~mL} / \mathrm{min} ; \lambda=$ $272 \mathrm{~nm} ; \mathrm{t}_{\mathrm{R} 1}($ major $)=8.8 \mathrm{~min} ; \mathrm{t}_{\mathrm{R} 2}($ minor $)=10.1 \mathrm{~min}$.

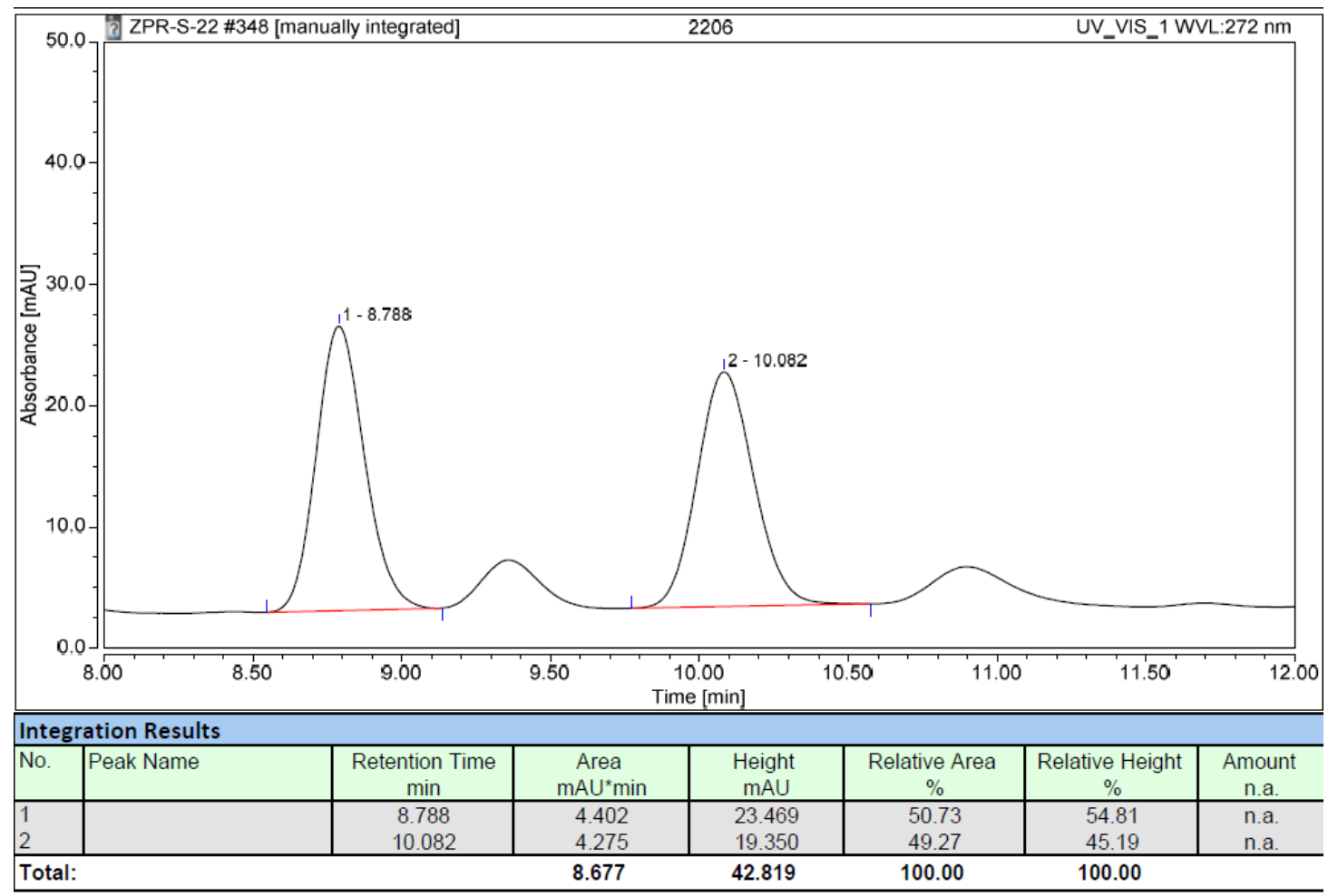




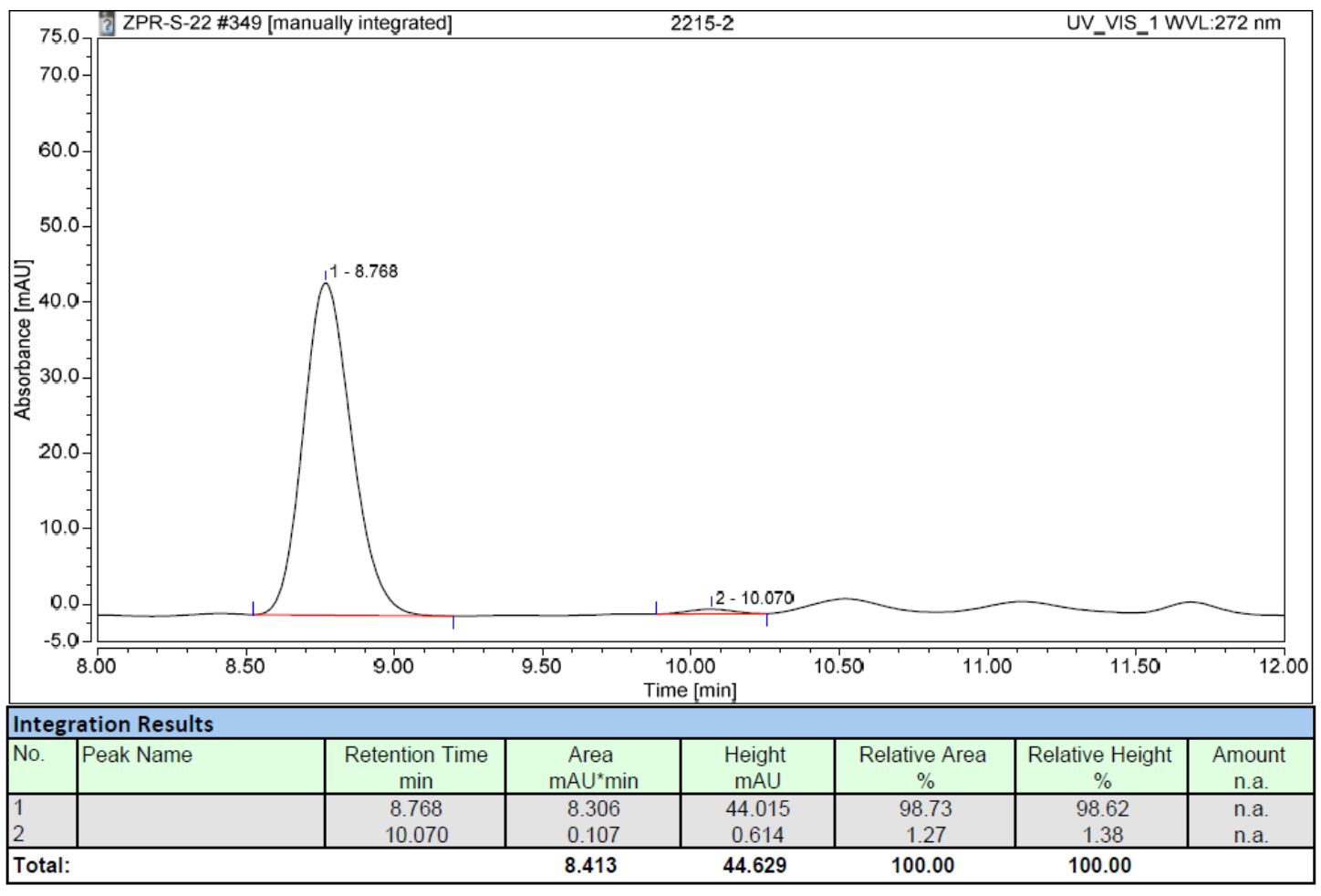




\section{X-Ray Data for $4 b$}<smiles>O=C1CCC[C@]2(O)[C@H](Cc3ccccc3)c3ccccc3C[C@]12Cc1ccccc1</smiles>

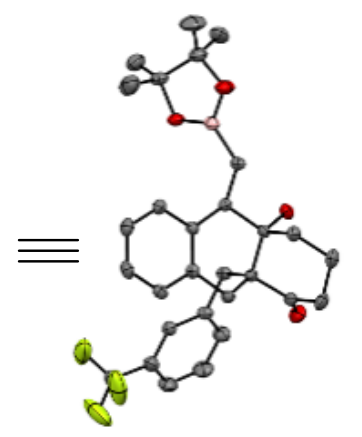

4b

Table S6. Crystal data and structure refinement for ga_90426d_a.

Identification code

Empirical formula

Formula weight

Temperature

Wavelength

Crystal system

Space group

Unit cell dimensions

Volume

Z

Density (calculated)

Absorption coefficient

$\mathrm{F}(000)$

Crystal size

Theta range for data collection

Index ranges

Reflections collected

Independent reflections

Completeness to theta $=53.594^{\circ}$

Absorption correction

Max. and min. transmission

Refinement method ga_90426d_a

C29 H34 B F3 O4

514.37

173(2) K

$1.34138 \AA$

Orthorhombic

P212121

$a=10.4164(3) \AA \quad \alpha=90^{\circ}$.

$\mathrm{b}=10.7443(4) \AA \quad \beta=90^{\circ}$.

$\mathrm{c}=23.0583(10) \AA \quad \gamma=90^{\circ}$.

2580.61(17) $\AA^{3}$

4

$1.324 \mathrm{Mg} / \mathrm{m}^{3}$

$0.532 \mathrm{~mm}^{-1}$

1088

$0.150 \times 0.050 \times 0.030 \mathrm{~mm}^{3}$

3.335 to $57.982^{\circ}$.

$-10<=\mathrm{h}<=13,-13<=\mathrm{k}<=9,-28<=1<=29$

19249

$5441[\mathrm{R}(\mathrm{int})=0.0300]$

$99.9 \%$

Semi-empirical from equivalents

0.752 and 0.668

Full-matrix least-squares on $\mathrm{F}^{2}$ 
Data / restraints / parameters

Goodness-of-fit on $\mathrm{F}^{2}$

Final R indices [I $>2 \operatorname{sigma}(\mathrm{I})]$

$\mathrm{R}$ indices (all data)

Absolute structure parameter

Extinction coefficient

Largest diff. peak and hole
$5441 / 0 / 342$

1.050

$$
\begin{aligned}
& \mathrm{R} 1=0.0416, \mathrm{wR} 2=0.1135 \\
& \mathrm{R} 1=0.0435, \mathrm{wR} 2=0.1156 \\
& -0.04(6) \\
& \mathrm{n} / \mathrm{a}
\end{aligned}
$$

0.393 and -0.225 e. $\AA^{-3}$

Table S7. Atomic coordinates ( $\mathrm{x} 10^{4}$ ) and equivalent isotropic displacement

\begin{tabular}{|c|c|c|c|c|}
\hline & $\mathrm{x}$ & $\mathrm{y}$ & $\mathrm{z}$ & $\mathrm{U}(\mathrm{eq})$ \\
\hline $\mathrm{B}(1)$ & $4588(3)$ & $5424(2)$ & $3588(1)$ & $25(1)$ \\
\hline $\mathrm{F}(1)$ & $3960(2)$ & $8103(3)$ & $7358(1)$ & $87(1)$ \\
\hline $\mathrm{F}(2)$ & $5806(3)$ & $7363(3)$ & $7274(1)$ & $87(1)$ \\
\hline $\mathrm{F}(3)$ & $5487(4)$ & 8891(3) & $7830(1)$ & $108(1)$ \\
\hline $\mathrm{O}(1)$ & $4413(2)$ & $9235(2)$ & $3942(1)$ & $26(1)$ \\
\hline $\mathrm{O}(2)$ & $3980(2)$ & $11470(2)$ & $5000(1)$ & $37(1)$ \\
\hline $\mathrm{O}(3)$ & $5623(2)$ & $4909(2)$ & $3864(1)$ & $30(1)$ \\
\hline $\mathrm{O}(4)$ & $4335(2)$ & $4882(2)$ & $3069(1)$ & $40(1)$ \\
\hline $\mathrm{C}(1)$ & $3603(2)$ & $8597(2)$ & $4351(1)$ & $21(1)$ \\
\hline$C(2)$ & $2241(2)$ & $8750(2)$ & $4107(1)$ & $26(1)$ \\
\hline $\mathrm{C}(3)$ & 1833(3) & $10111(2)$ & $4054(1)$ & $35(1)$ \\
\hline $\mathrm{C}(4)$ & 1967(3) & $10803(2)$ & $4630(1)$ & $35(1)$ \\
\hline$C(5)$ & $3288(3)$ & $10606(2)$ & $4881(1)$ & $26(1)$ \\
\hline$C(6)$ & $3716(2)$ & $9247(2)$ & $4959(1)$ & $20(1)$ \\
\hline$C(7)$ & $2805(2)$ & $8581(2)$ & $5378(1)$ & $22(1)$ \\
\hline $\mathrm{C}(8)$ & $3013(2)$ & $7189(2)$ & $5397(1)$ & $21(1)$ \\
\hline $\mathrm{C}(9)$ & $2660(2)$ & $6546(2)$ & $5898(1)$ & $25(1)$ \\
\hline$C(10)$ & 2791(2) & $5262(2)$ & $5935(1)$ & $28(1)$ \\
\hline
\end{tabular}
parameters $\left(\AA^{2} \times 10^{3}\right)$

for ga_90426d_a. U(eq) is defined as one third of the trace of the orthogonalized Uij tensor. 


$\begin{array}{lrrrr}\mathrm{C}(11) & 3263(3) & 4608(2) & 5463(1) & 30(1) \\ \mathrm{C}(12) & 3618(2) & 5246(2) & 4965(1) & 26(1) \\ \mathrm{C}(13) & 3515(2) & 6541(2) & 4921(1) & 20(1) \\ \mathrm{C}(14) & 4026(2) & 7210(2) & 4384(1) & 20(1) \\ \mathrm{C}(15) & 5118(2) & 9203(2) & 5183(1) & 22(1) \\ \mathrm{C}(16) & 5368(2) & 9657(2) & 5794(1) & 24(1) \\ \mathrm{C}(17) & 5156(2) & 8891(2) & 6269(1) & 25(1) \\ \mathrm{C}(18) & 5446(3) & 9295(3) & 6826(1) & 32(1) \\ \mathrm{C}(19) & 5970(3) & 10459(3) & 6919(1) & 41(1) \\ \mathrm{C}(20) & 6201(3) & 11221(3) & 6452(1) & 42(1) \\ \mathrm{C}(21) & 5901(3) & 10828(2) & 5894(1) & 34(1) \\ \mathrm{C}(22) & 5192(3) & 8431(3) & 7320(1) & 44(1) \\ \mathrm{C}(23) & 3742(2) & 6539(2) & 3806(1) & 24(1) \\ \mathrm{C}(24) & 5934(3) & 3753(2) & 3550(1) & 34(1) \\ \mathrm{C}(25) & 5380(3) & 4015(3) & 2942(1) & 37(1) \\ \mathrm{C}(26) & 7354(3) & 3519(3) & 3583(1) & 46(1) \\ \mathrm{C}(27) & 5213(4) & 2705(3) & 3866(2) & 58(1) \\ \mathrm{C}(28) & 6332(4) & 4682(3) & 2548(1) & 49(1) \\ \mathrm{C}(29) & 4817(4) & 2914(4) & 2625(2) & 56(1)\end{array}$




\section{Spectroscopic Data (NMR Spectrum)}

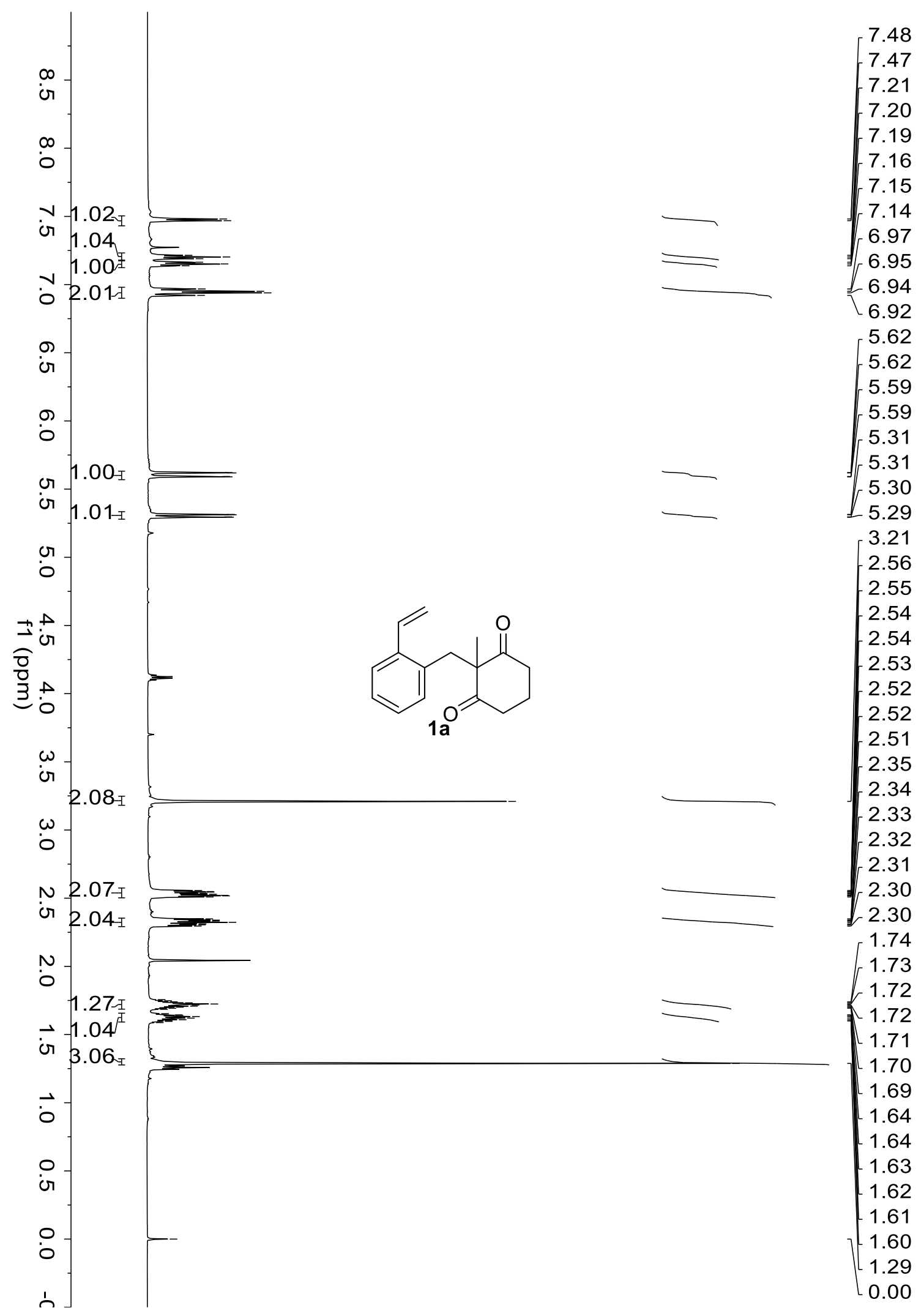




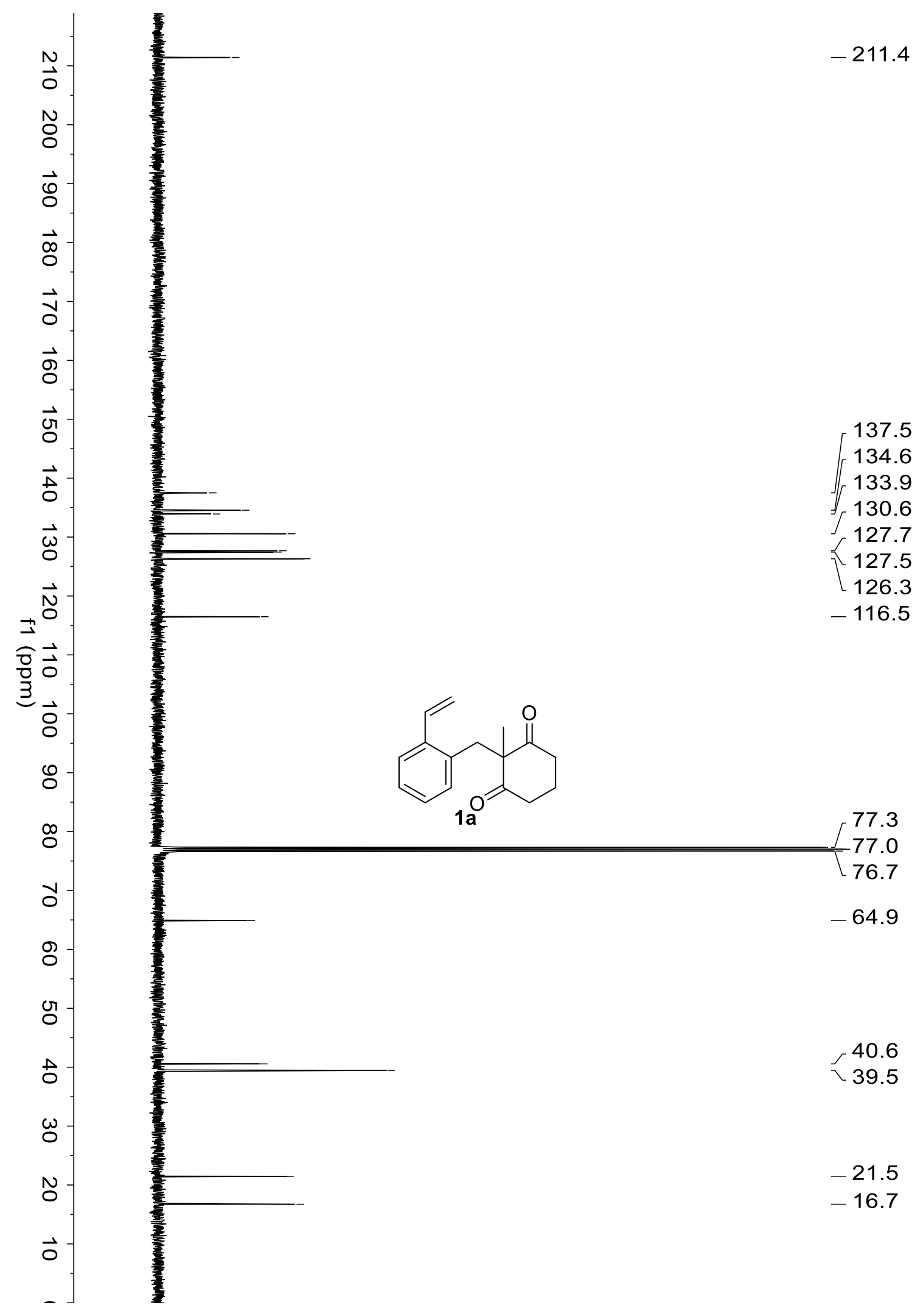




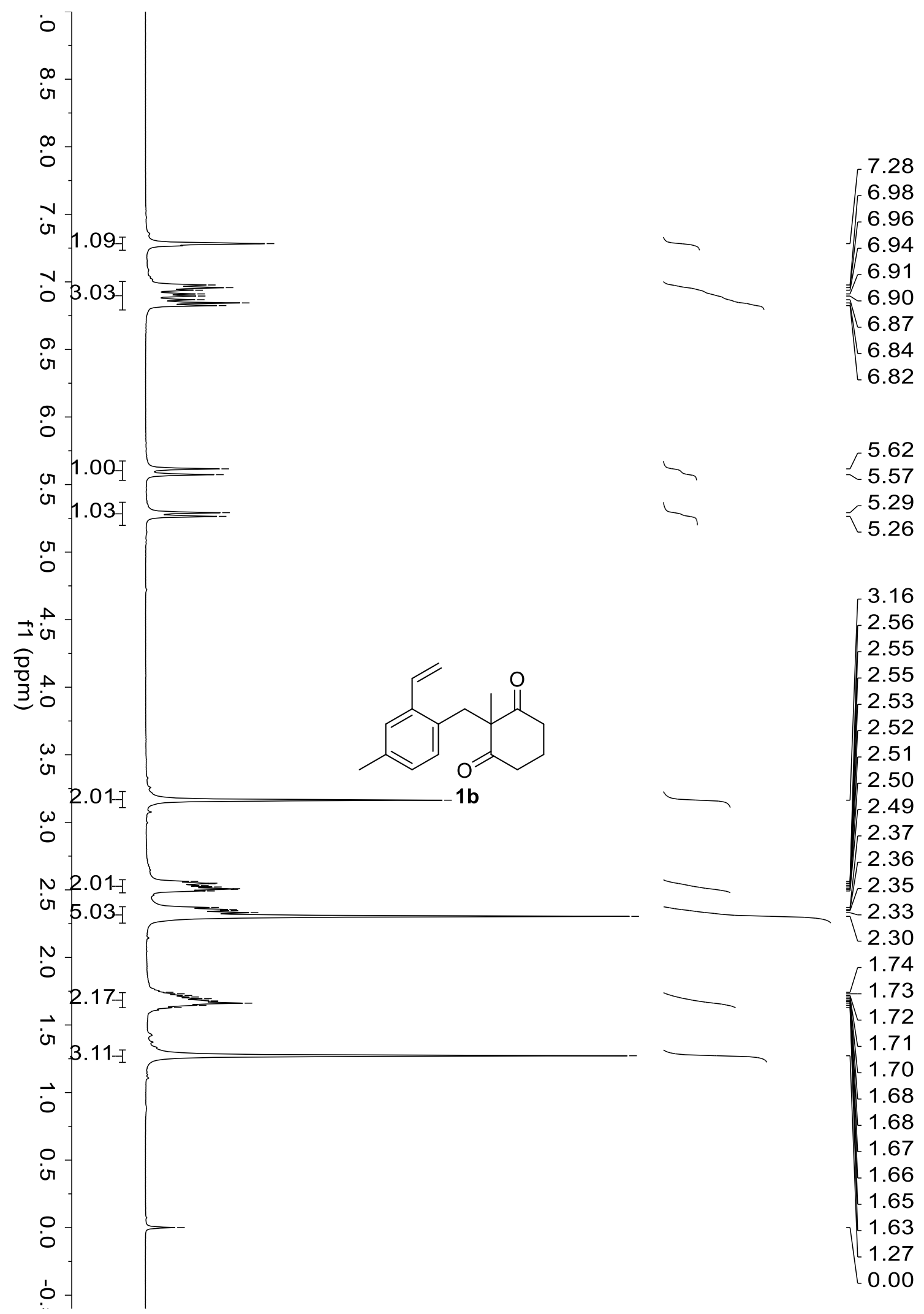




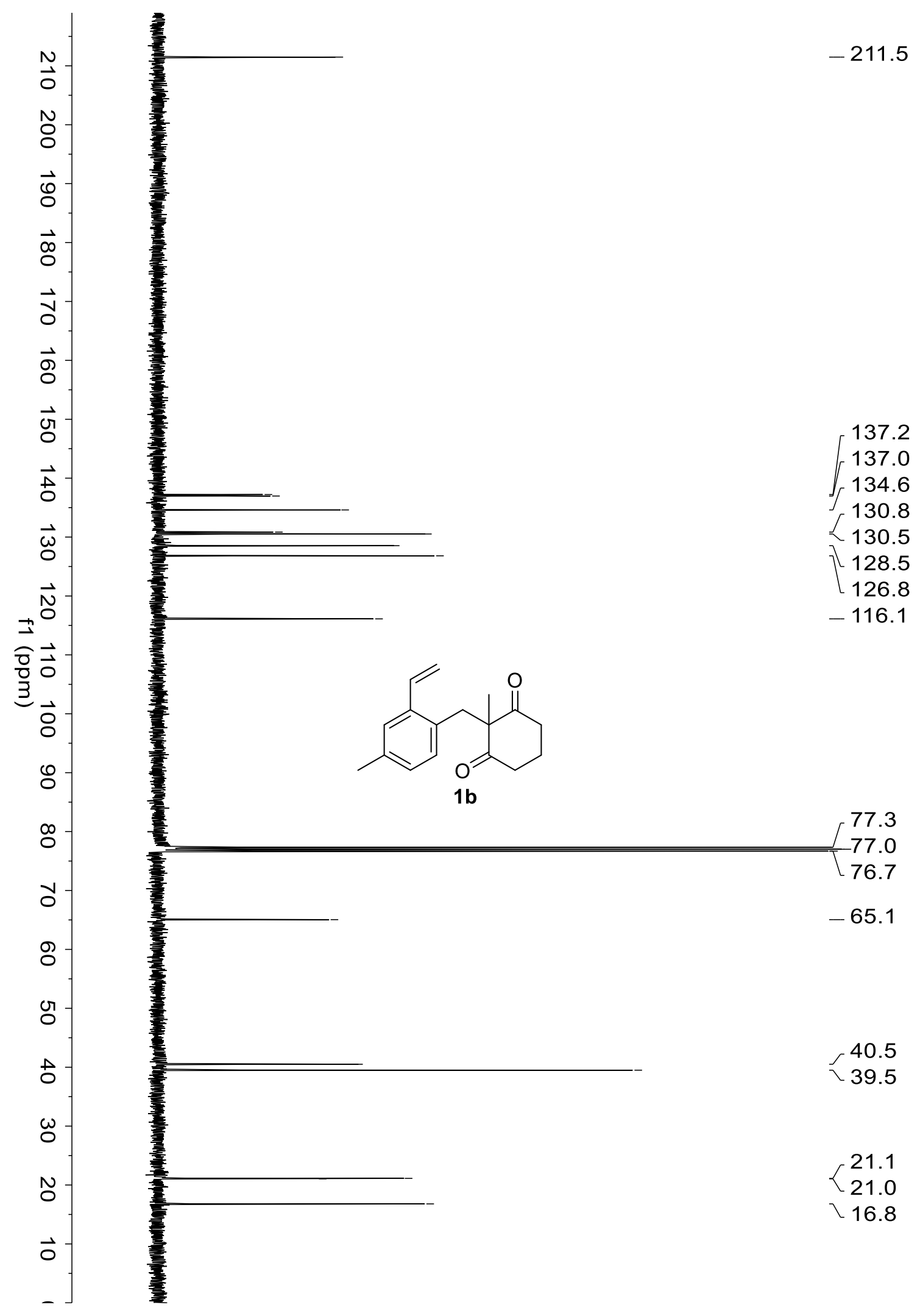



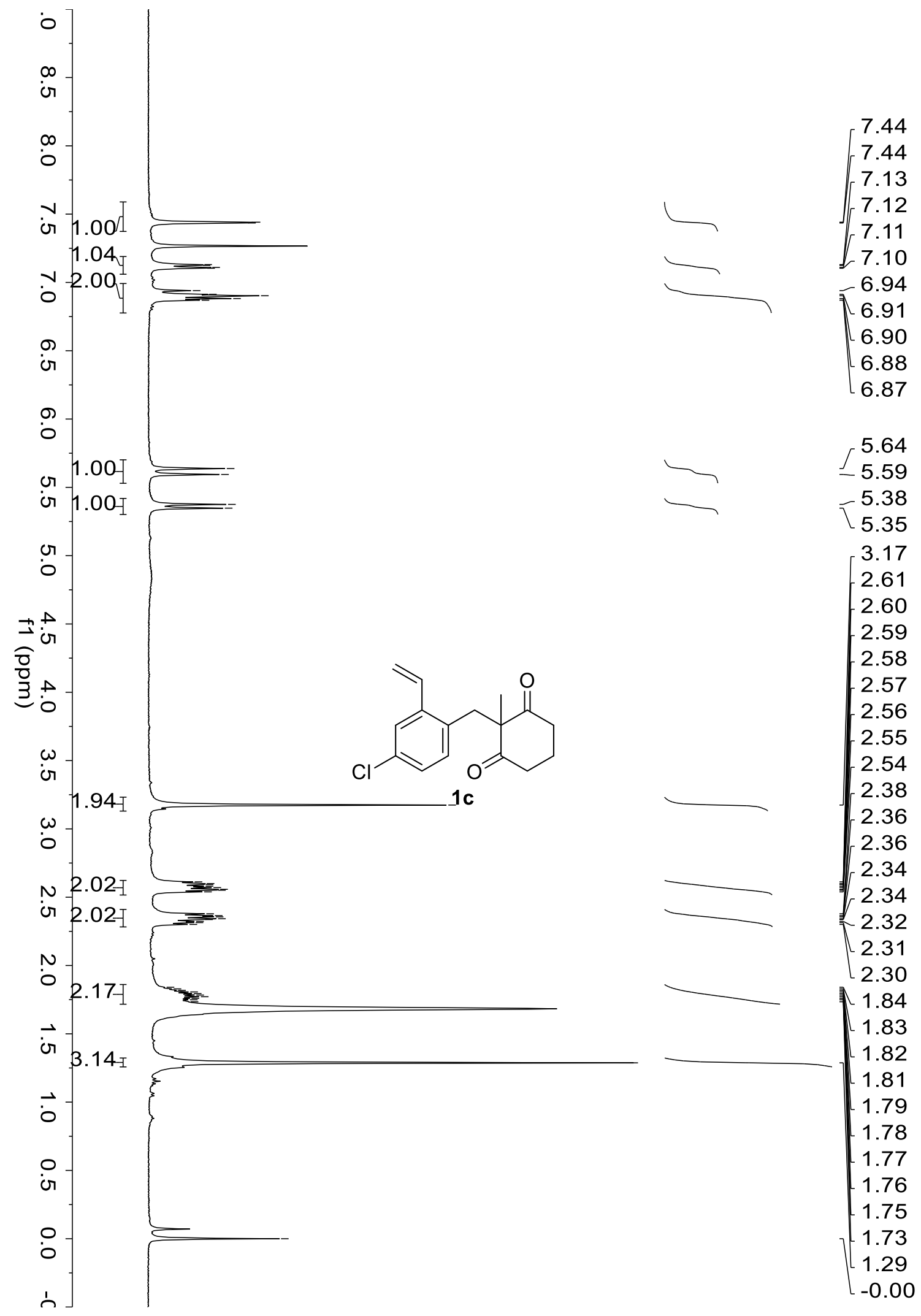


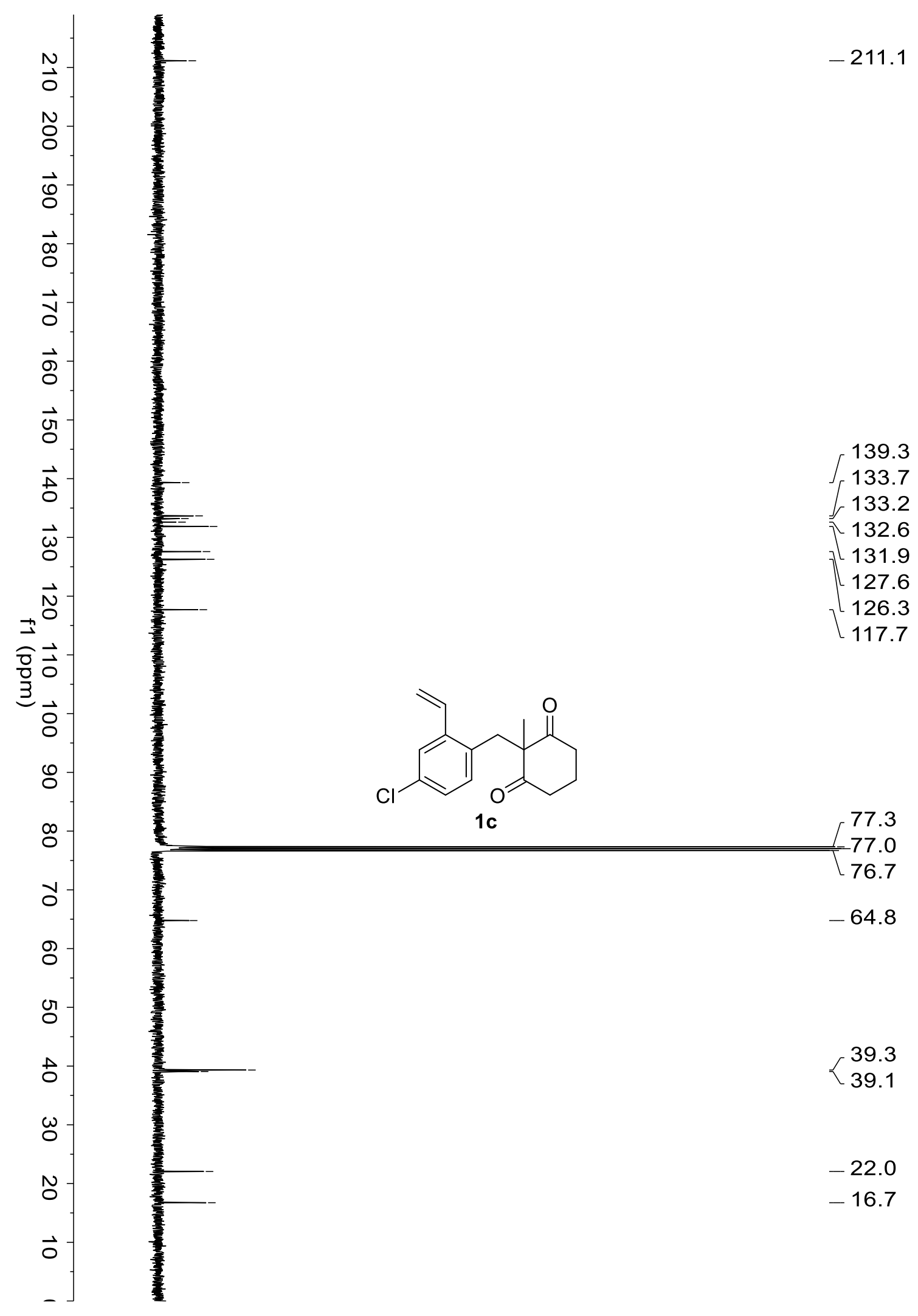




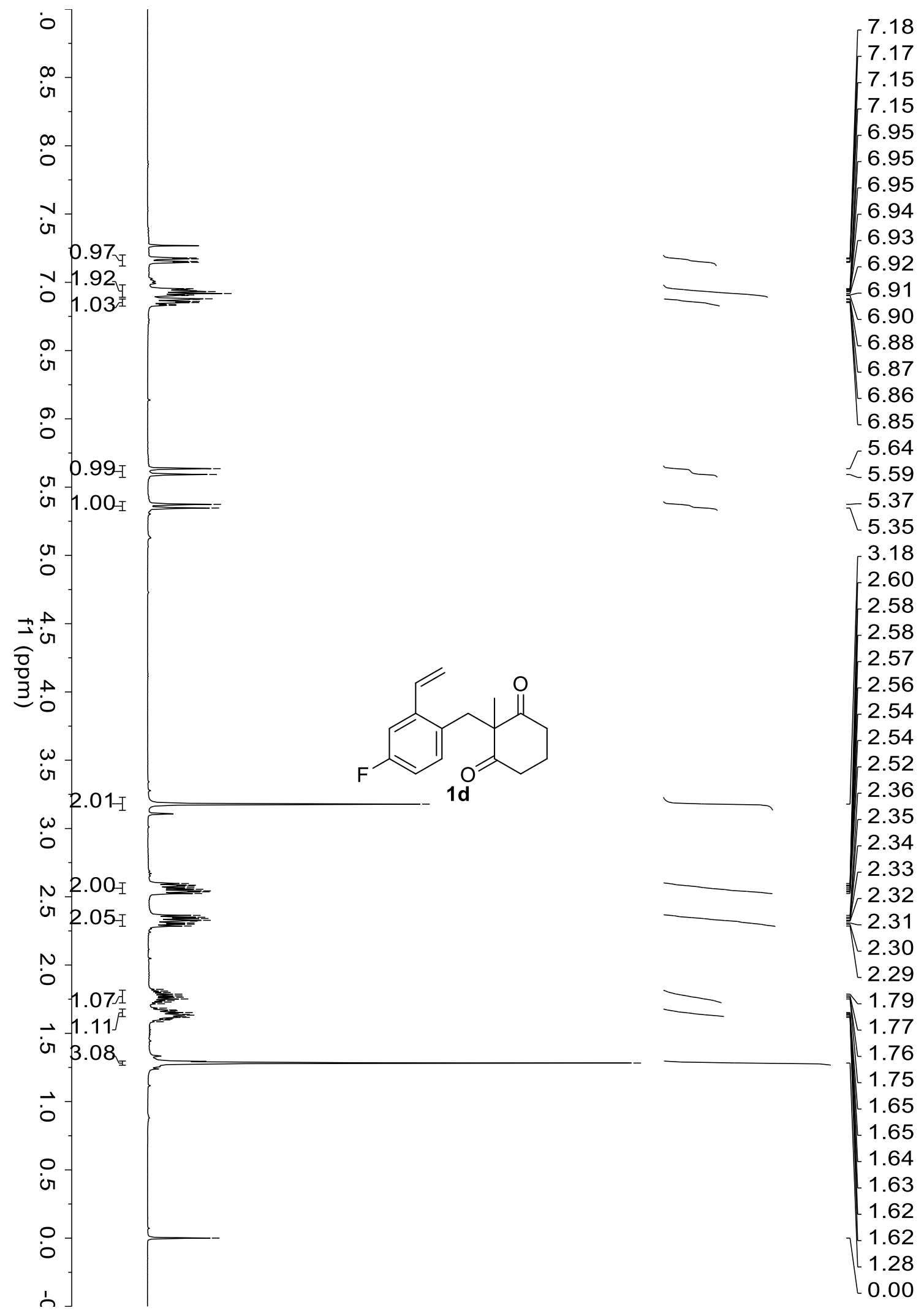




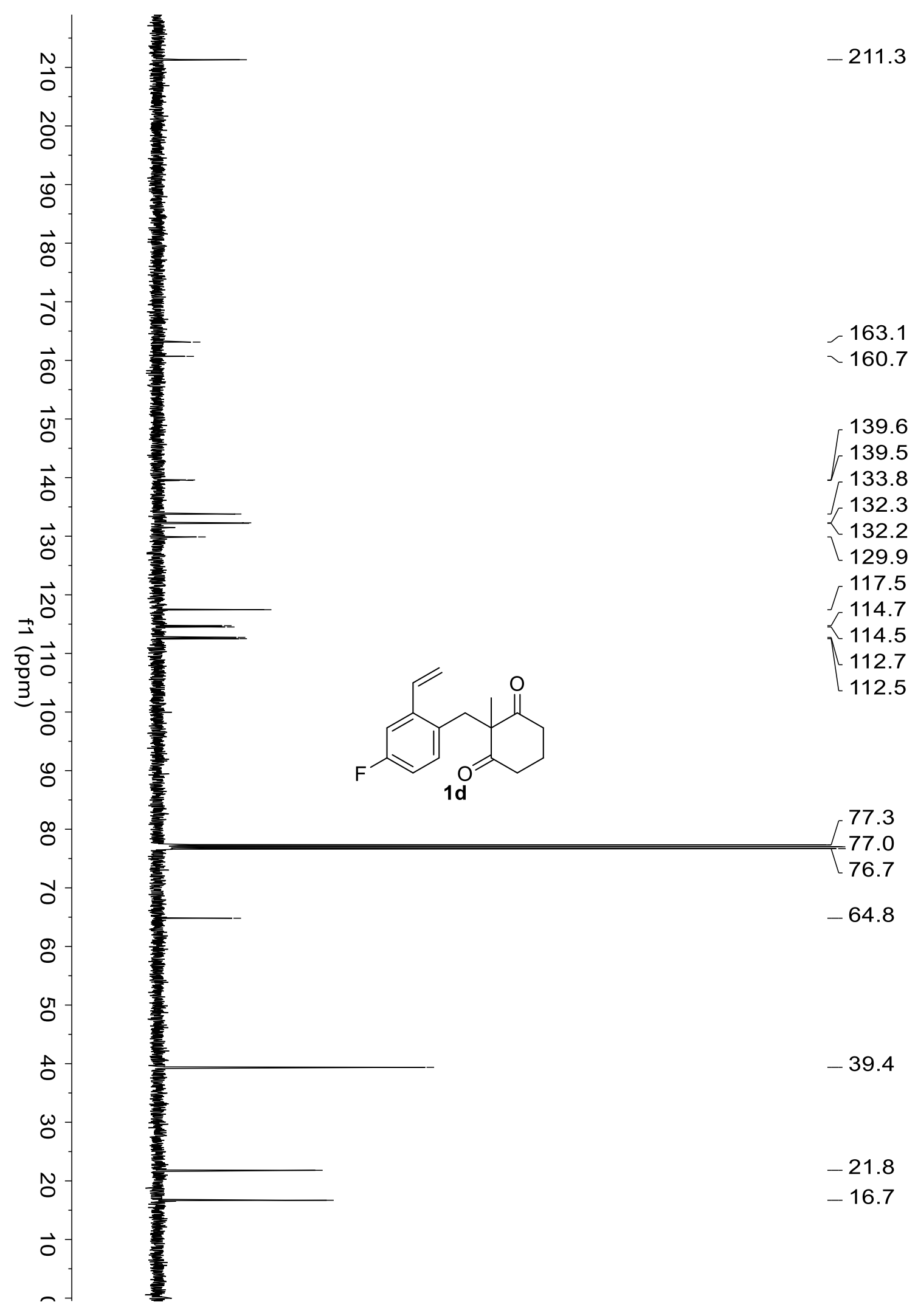



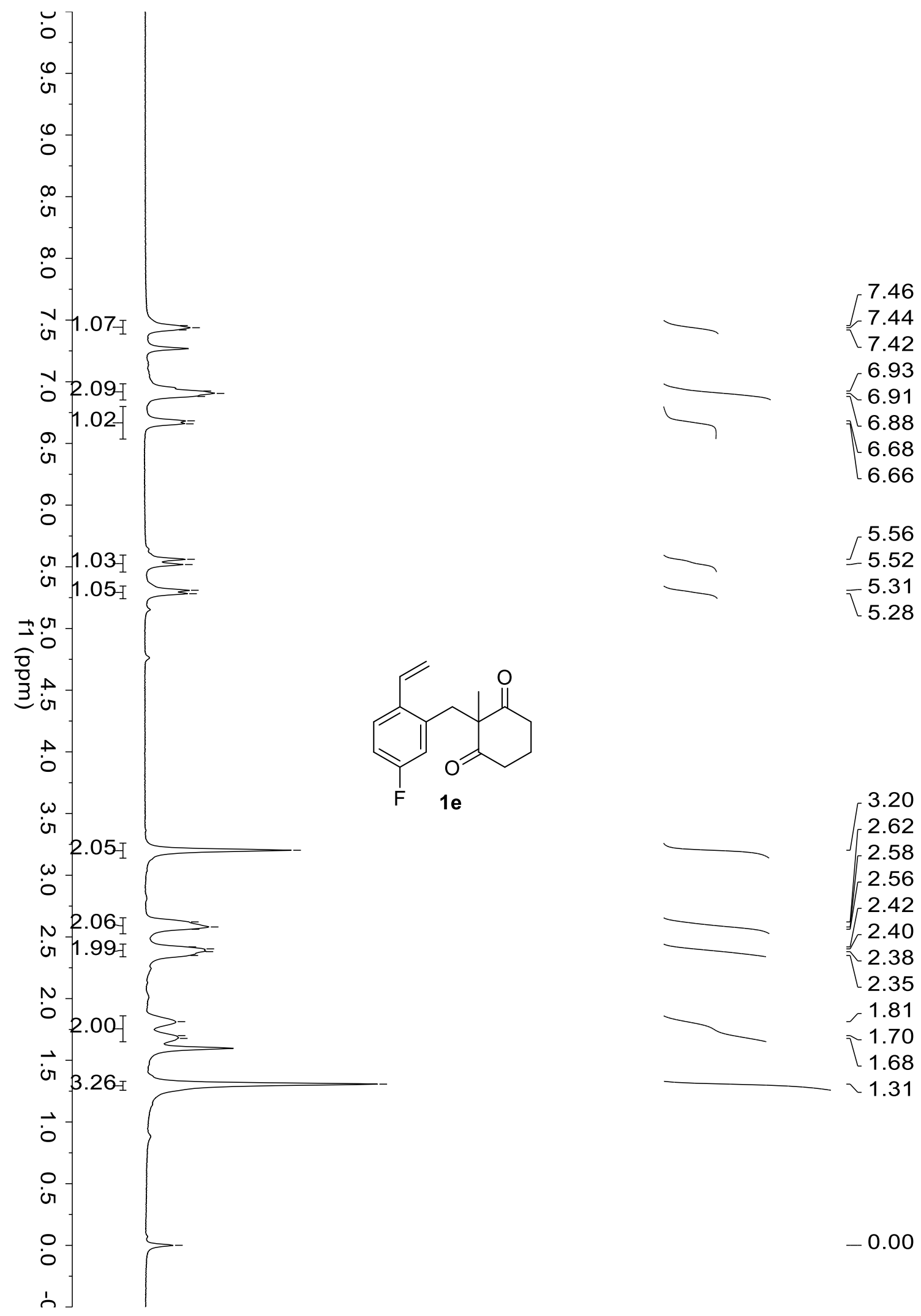

$-0.00$ 


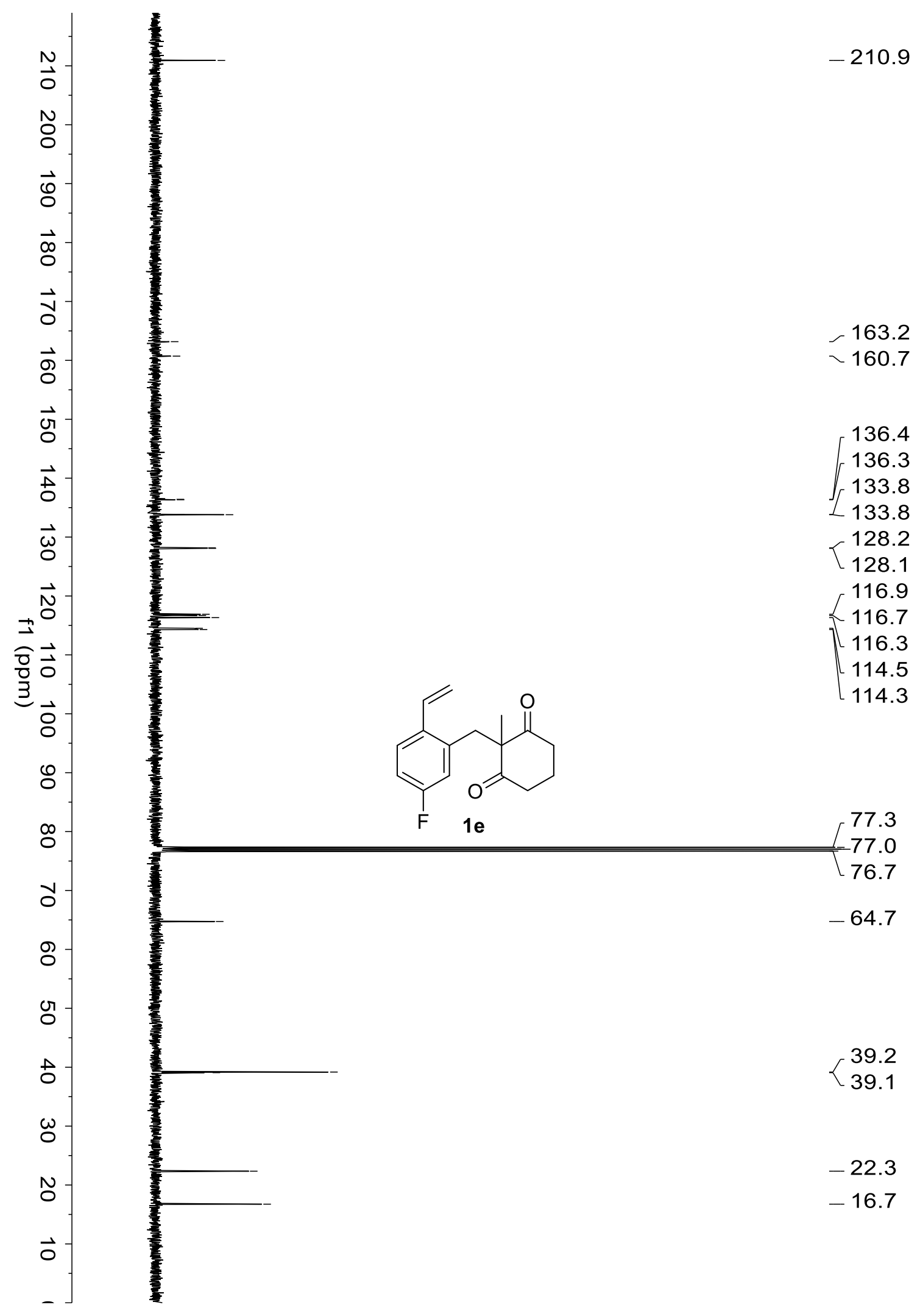




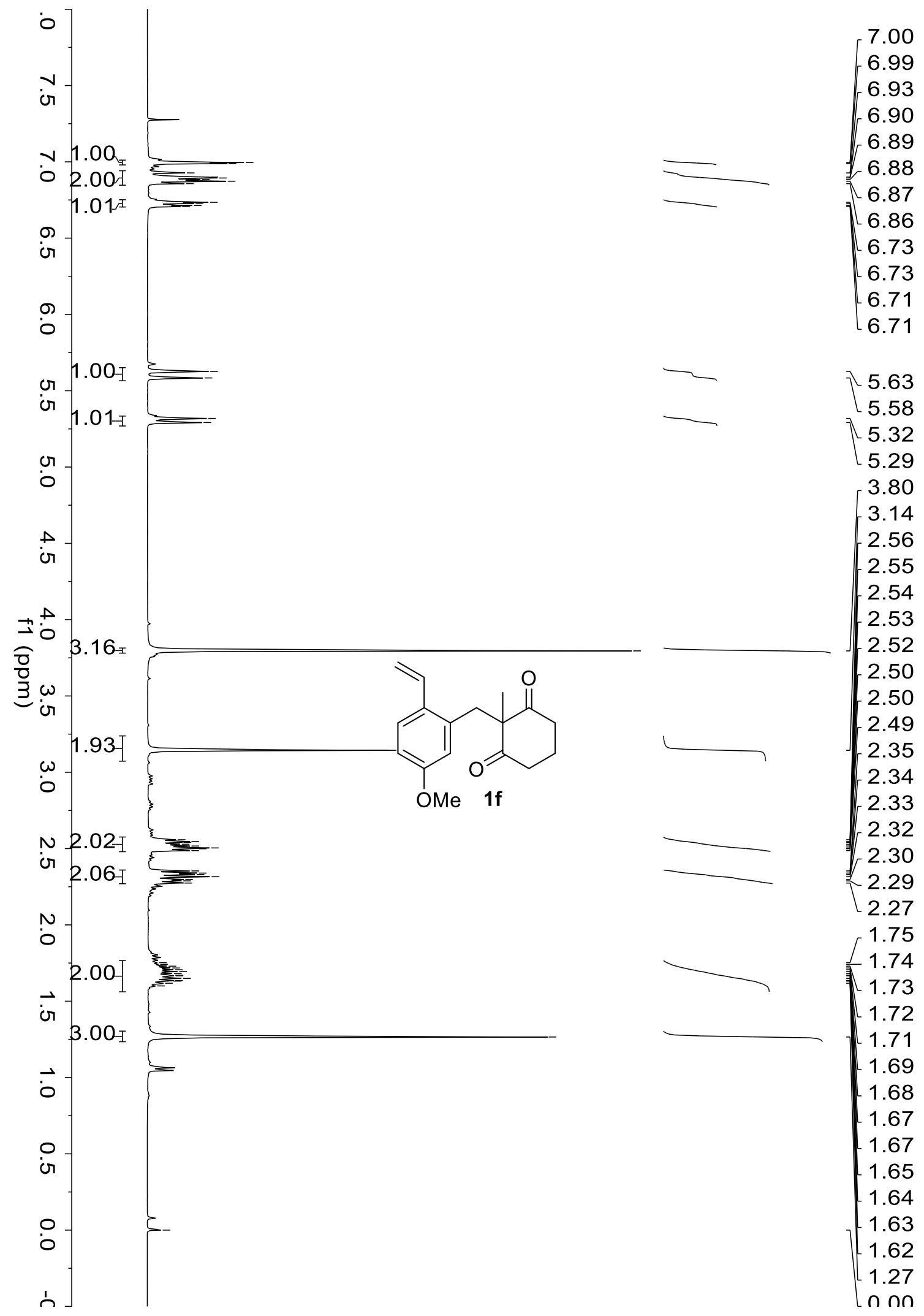




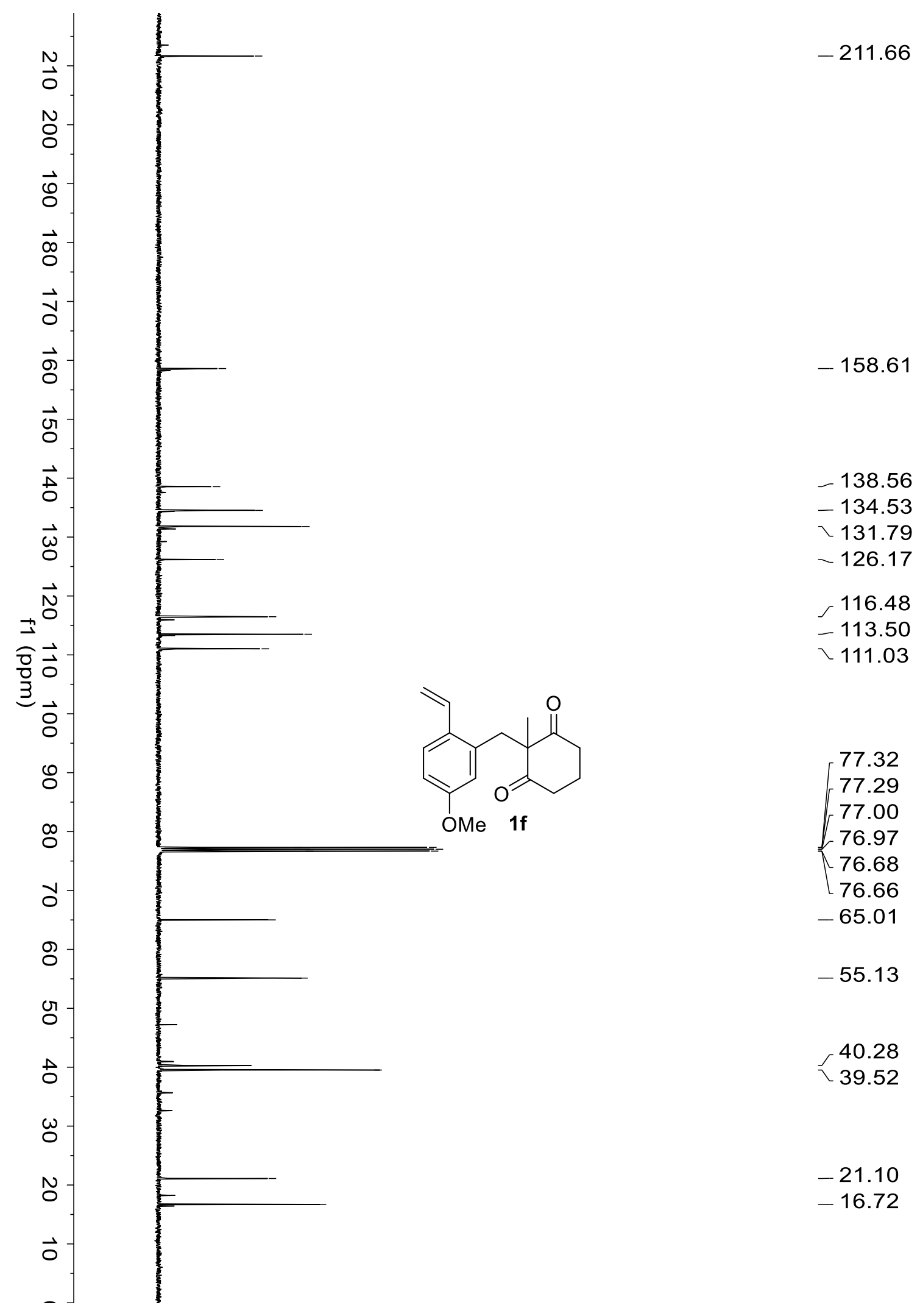




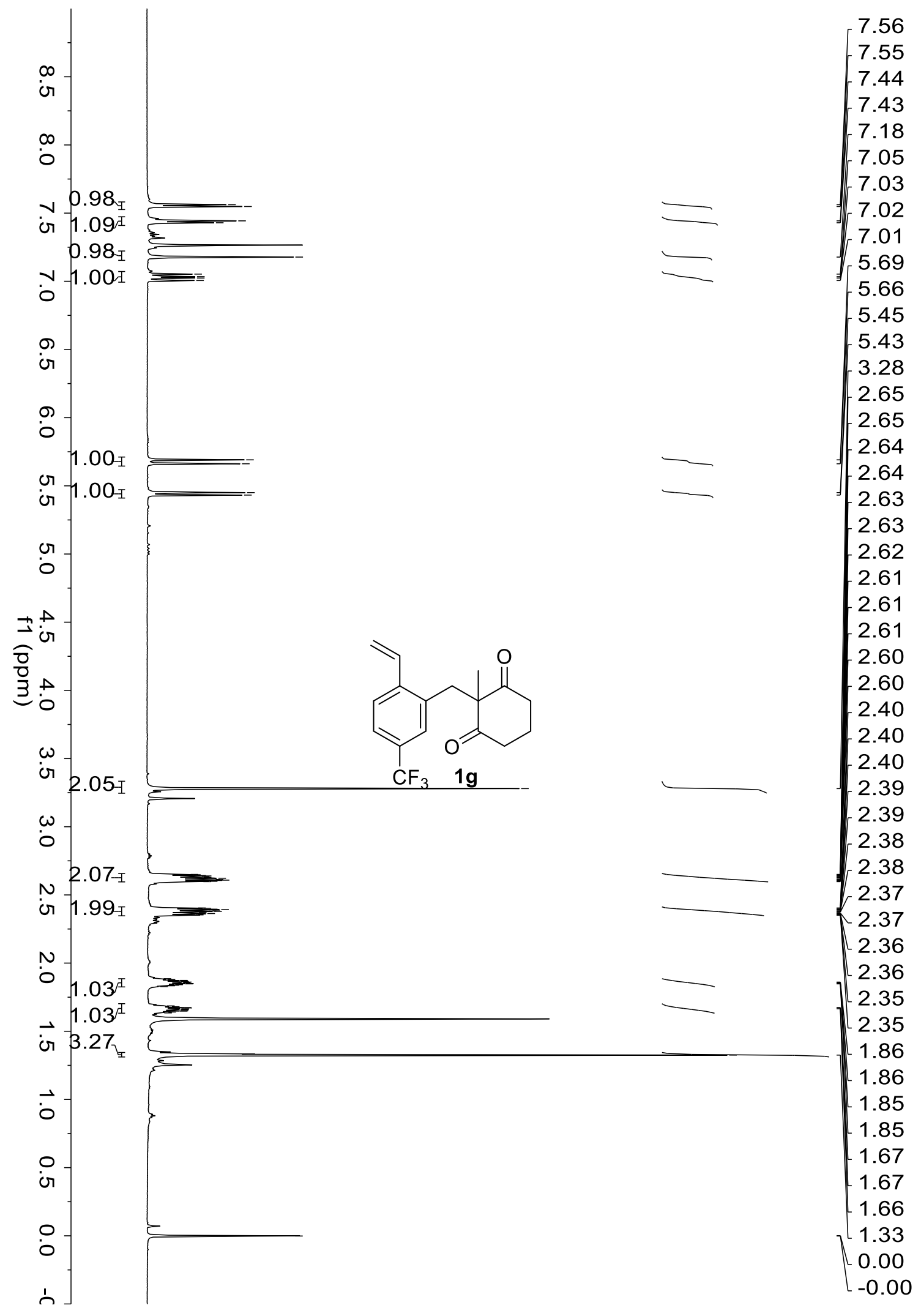




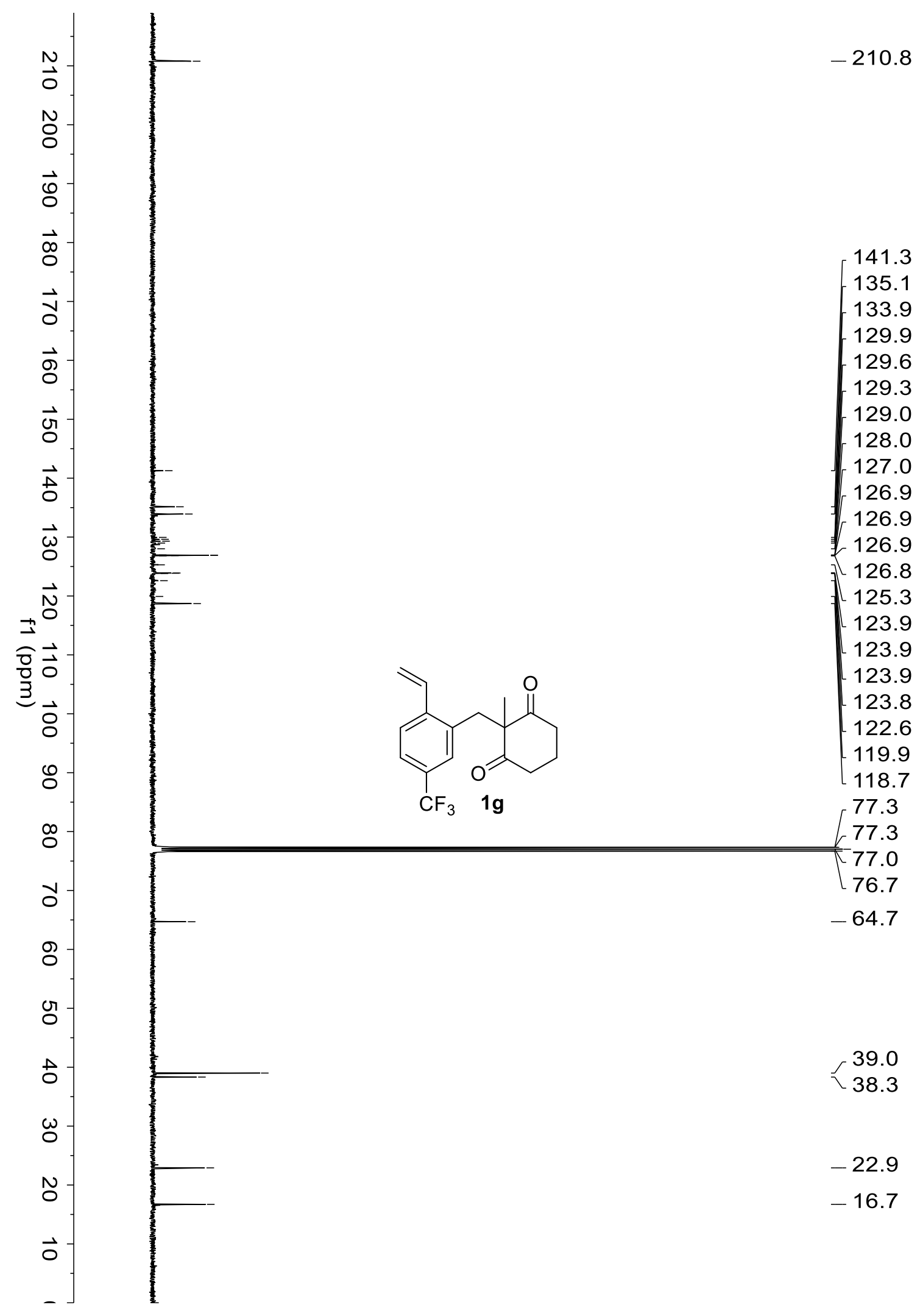




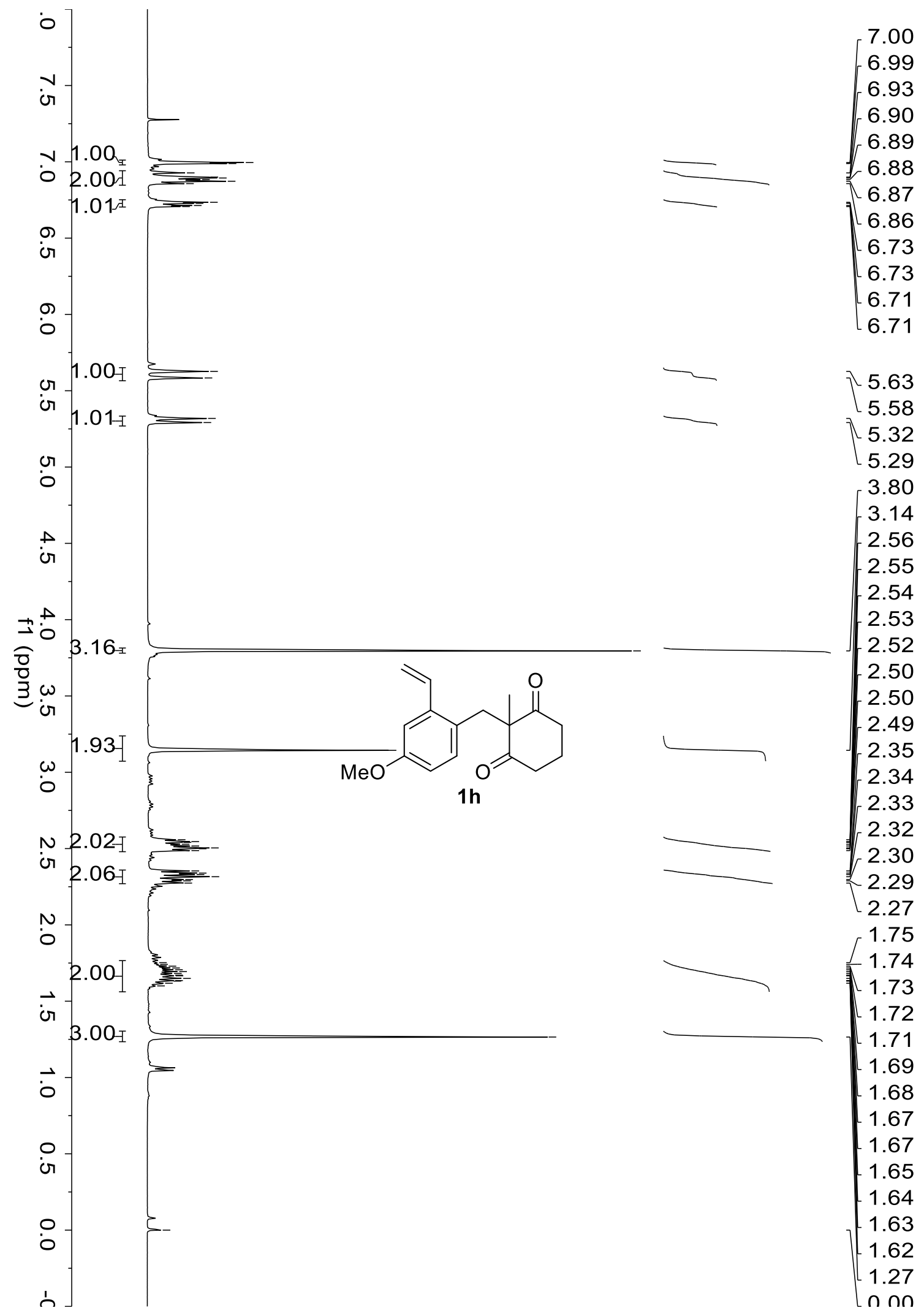




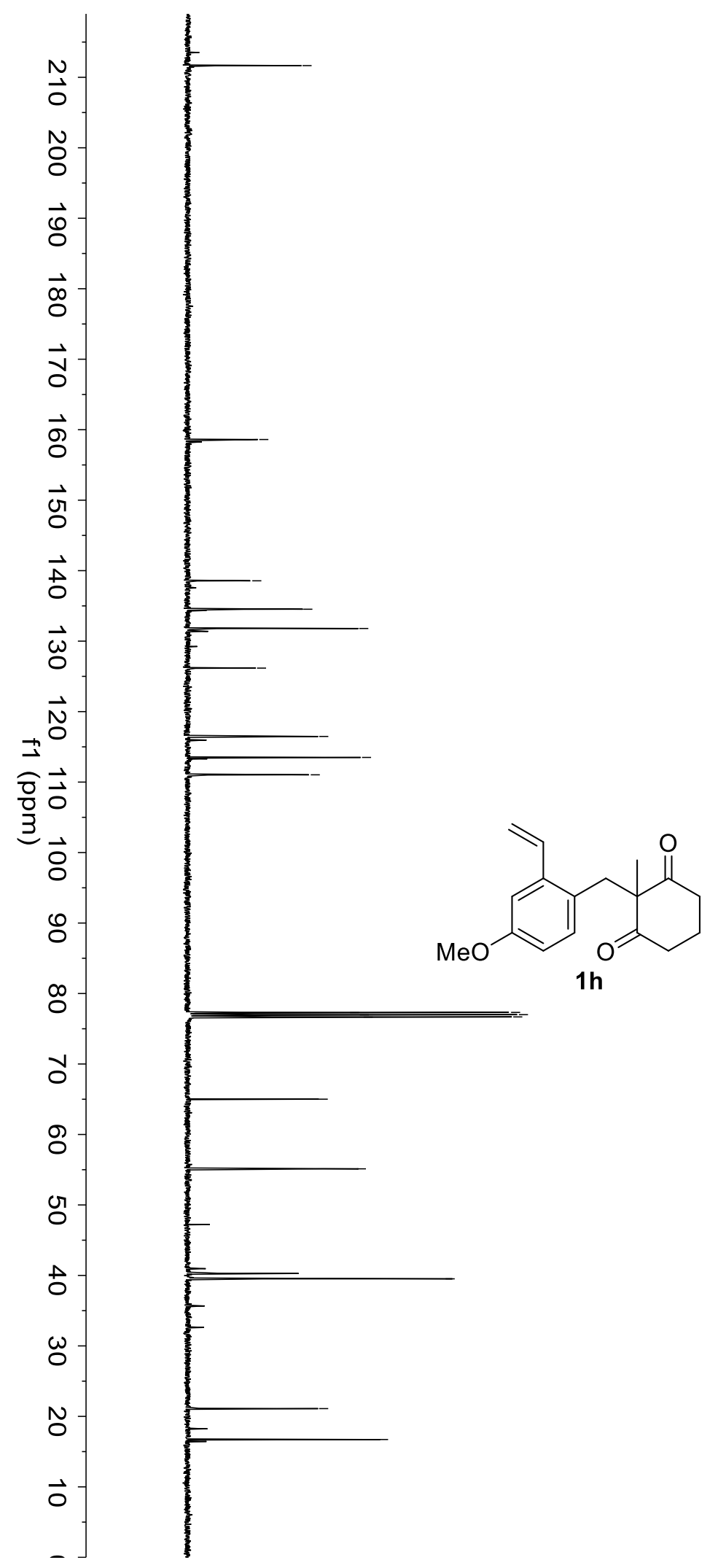

$-211.66$

$-158.61$

$-138.56$

$-134.53$

$\backslash 131.79$

$-126.17$

116.48

$-113.50$

$\backslash 111.03$

$\left\{\begin{array}{l}77.32 \\ 77.29 \\ 77.00 \\ 76.97 \\ 76.68 \\ 76.66\end{array}\right.$

$-65.01$

$-55.13$

40.28

$\backslash 39.52$

$-21.10$

$-16.72$ 


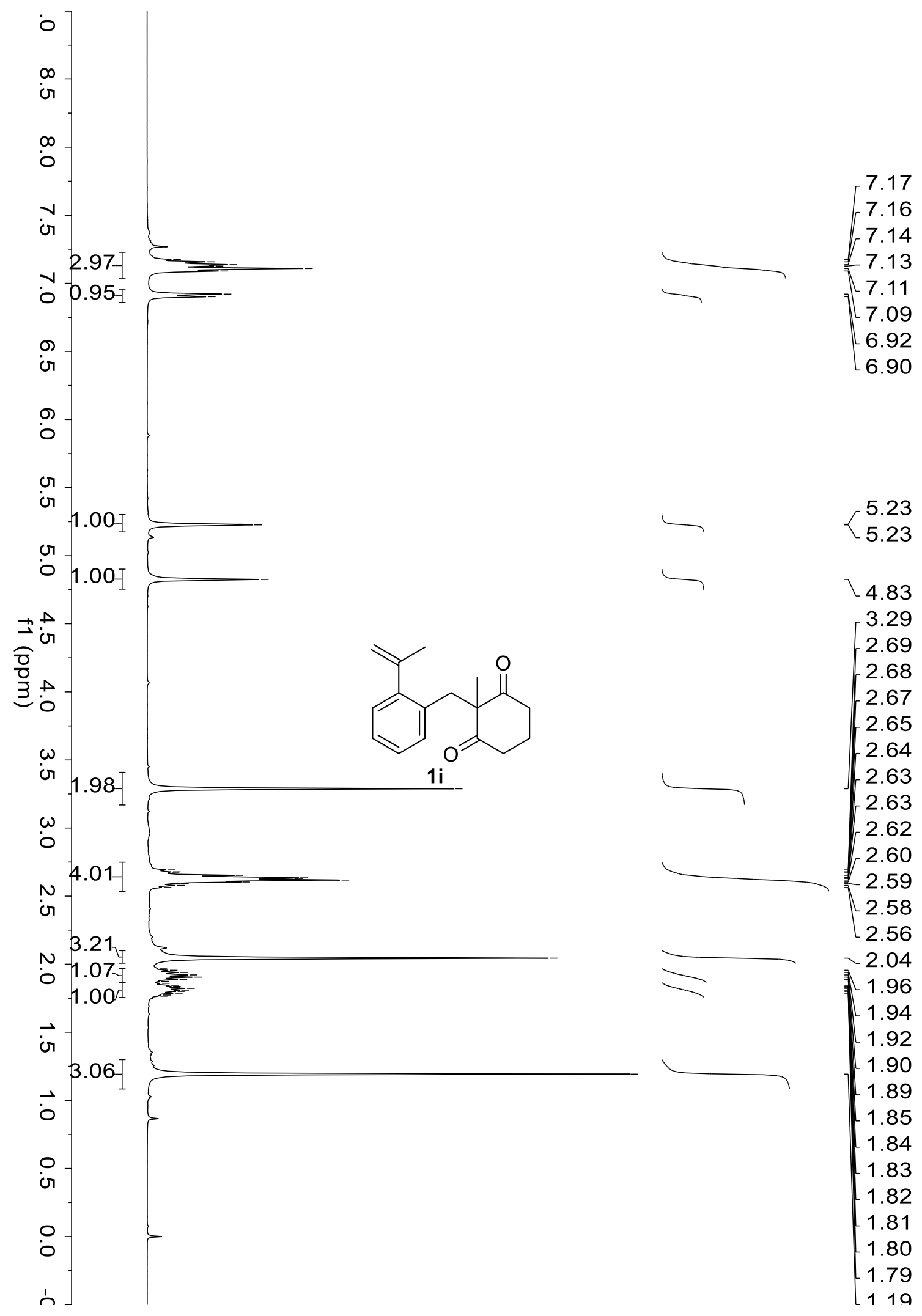




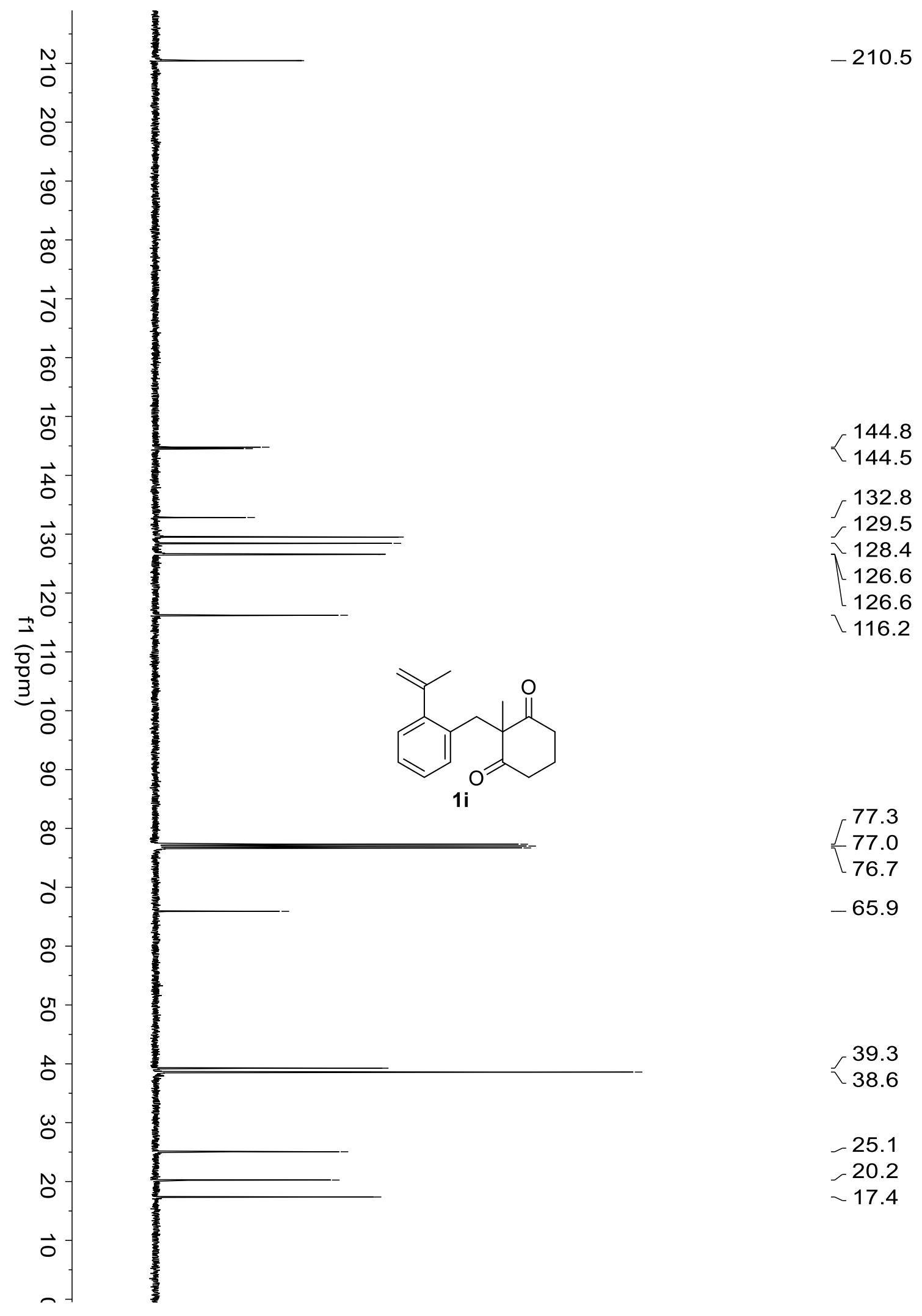




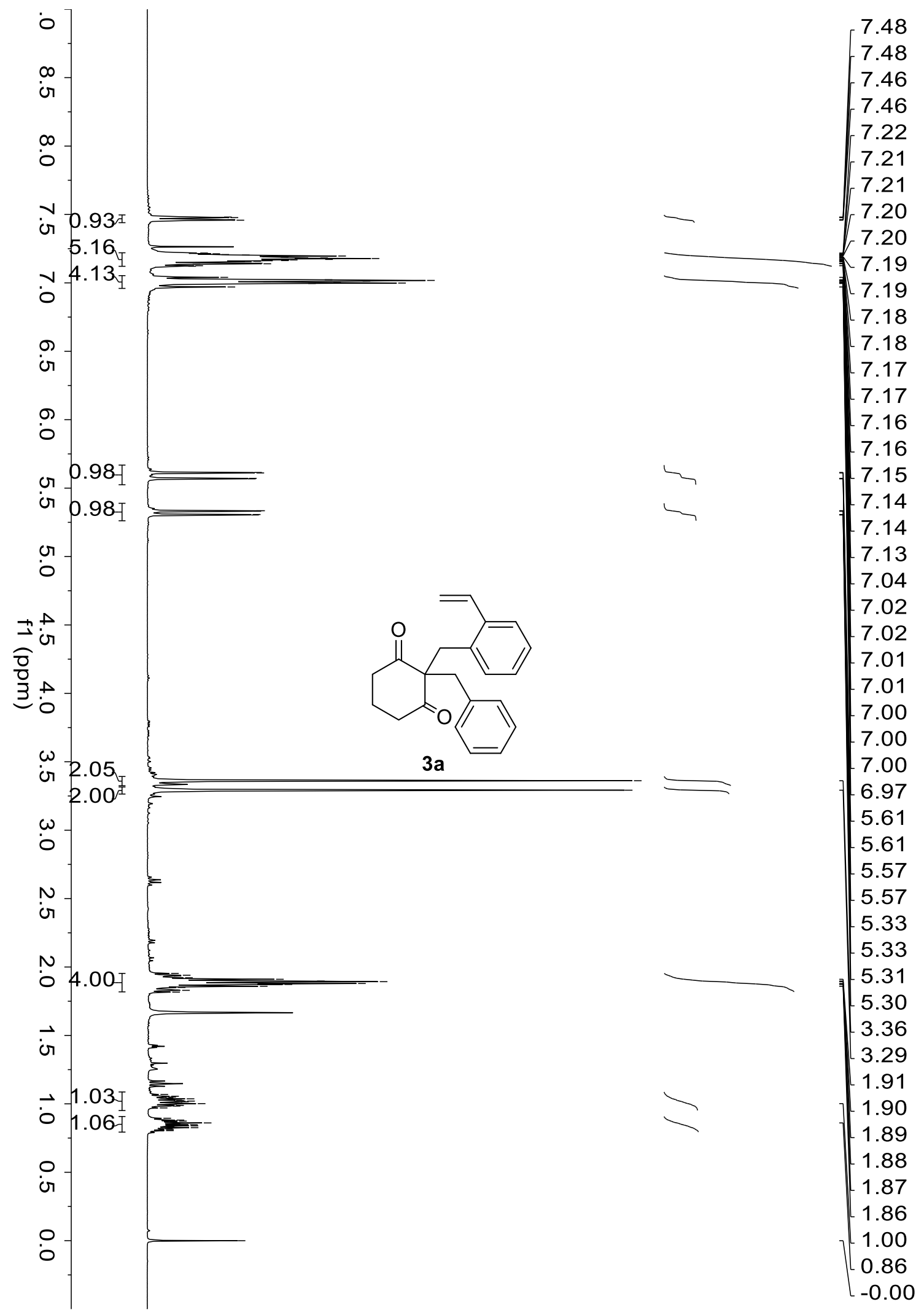




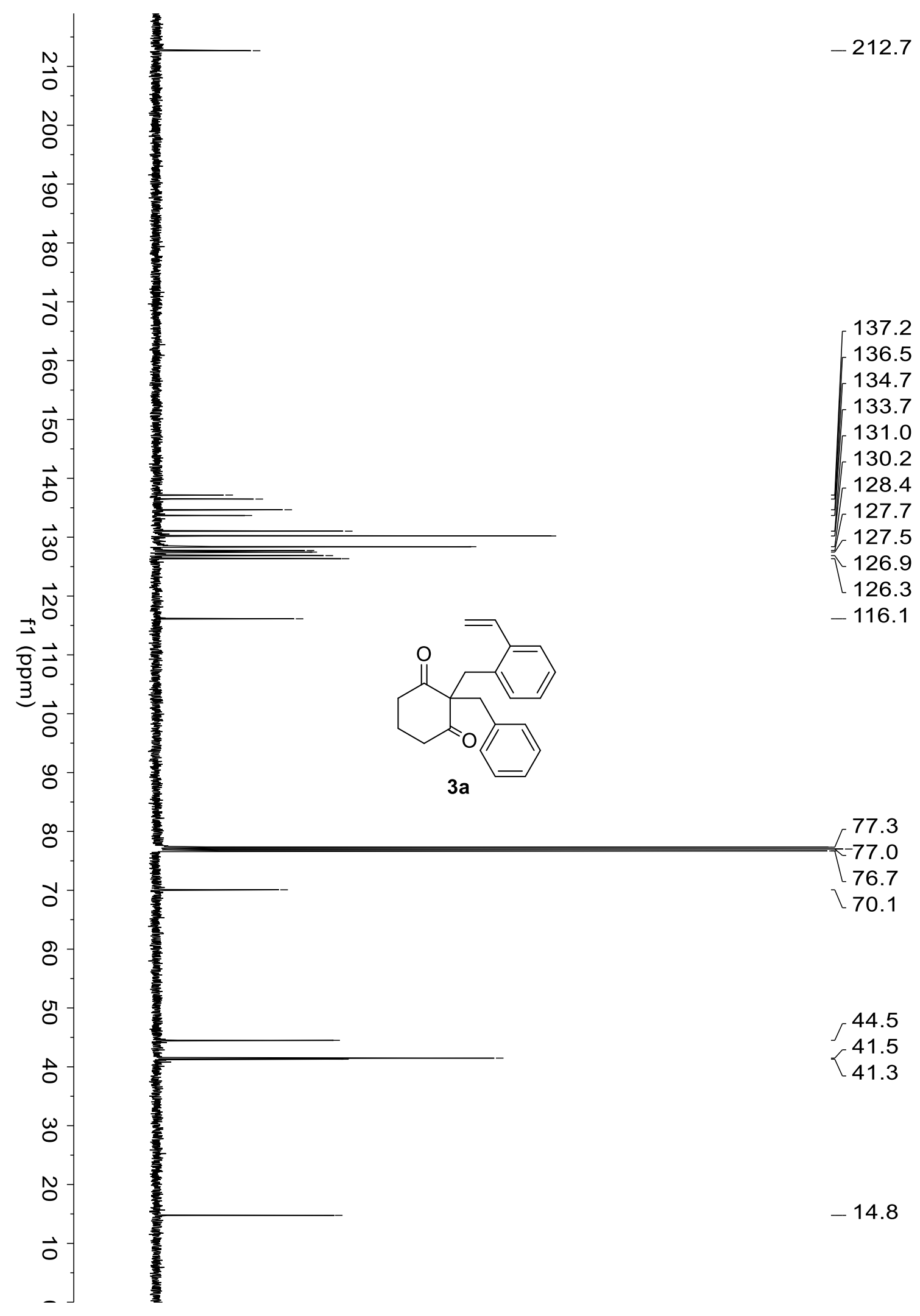




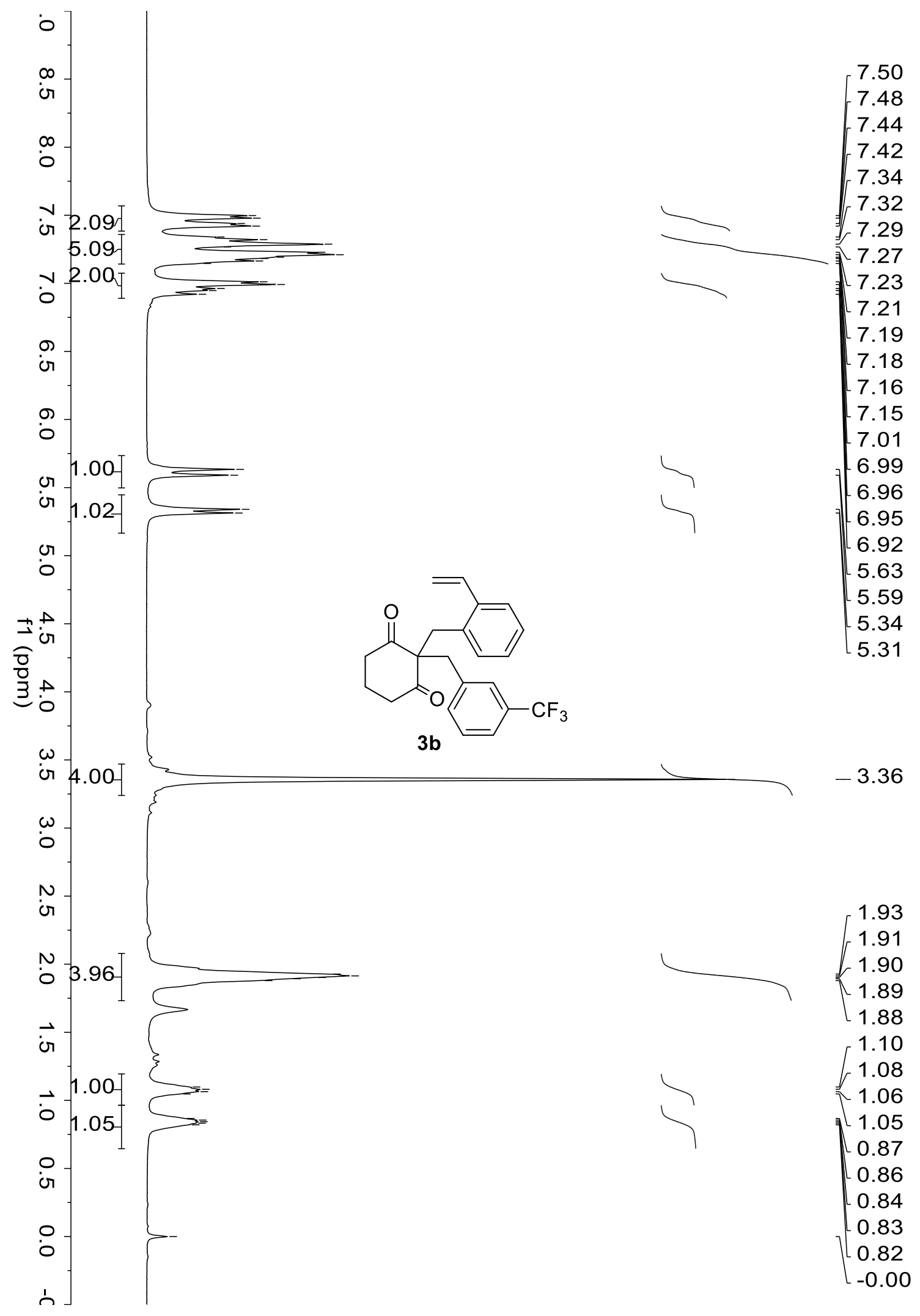




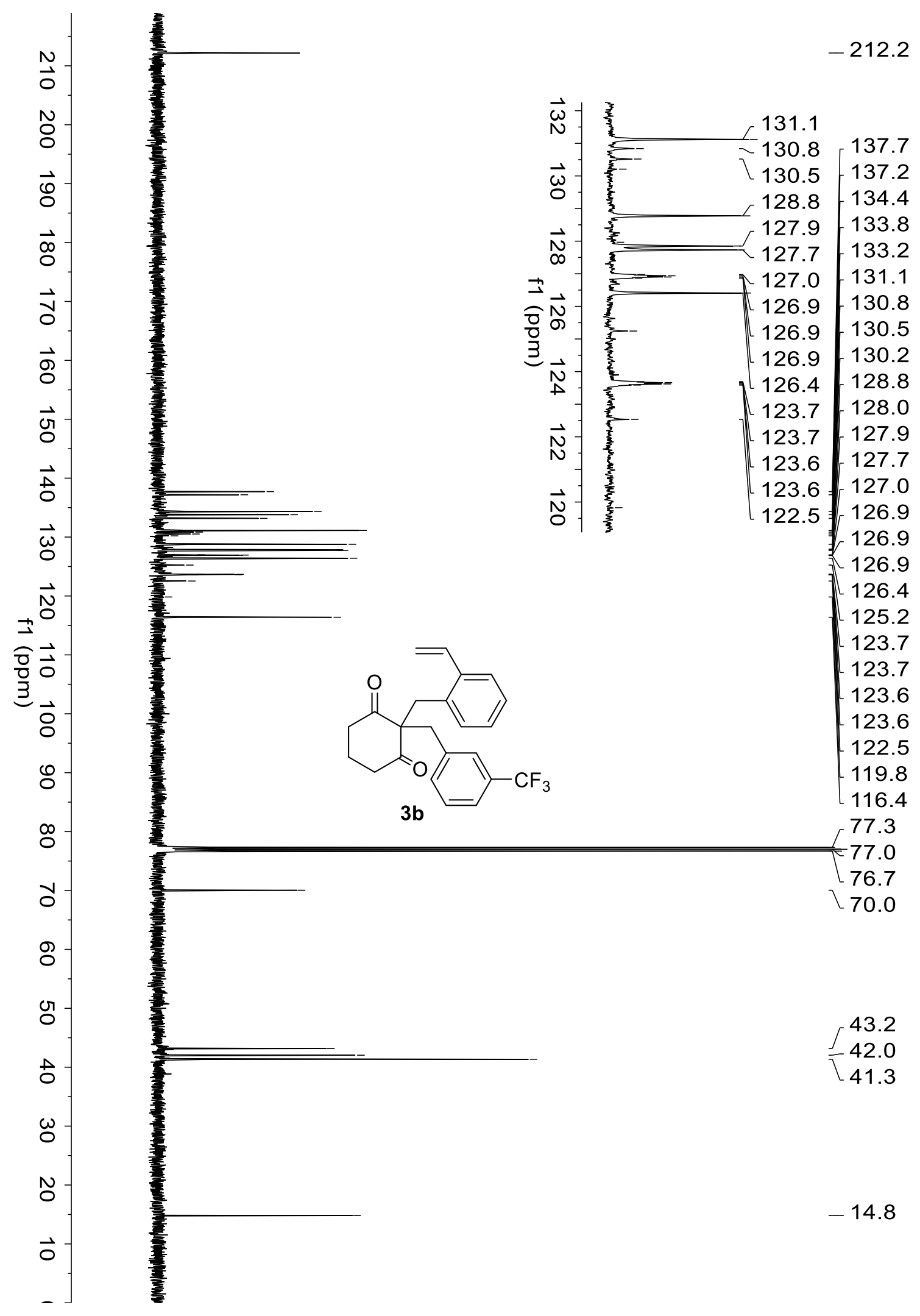




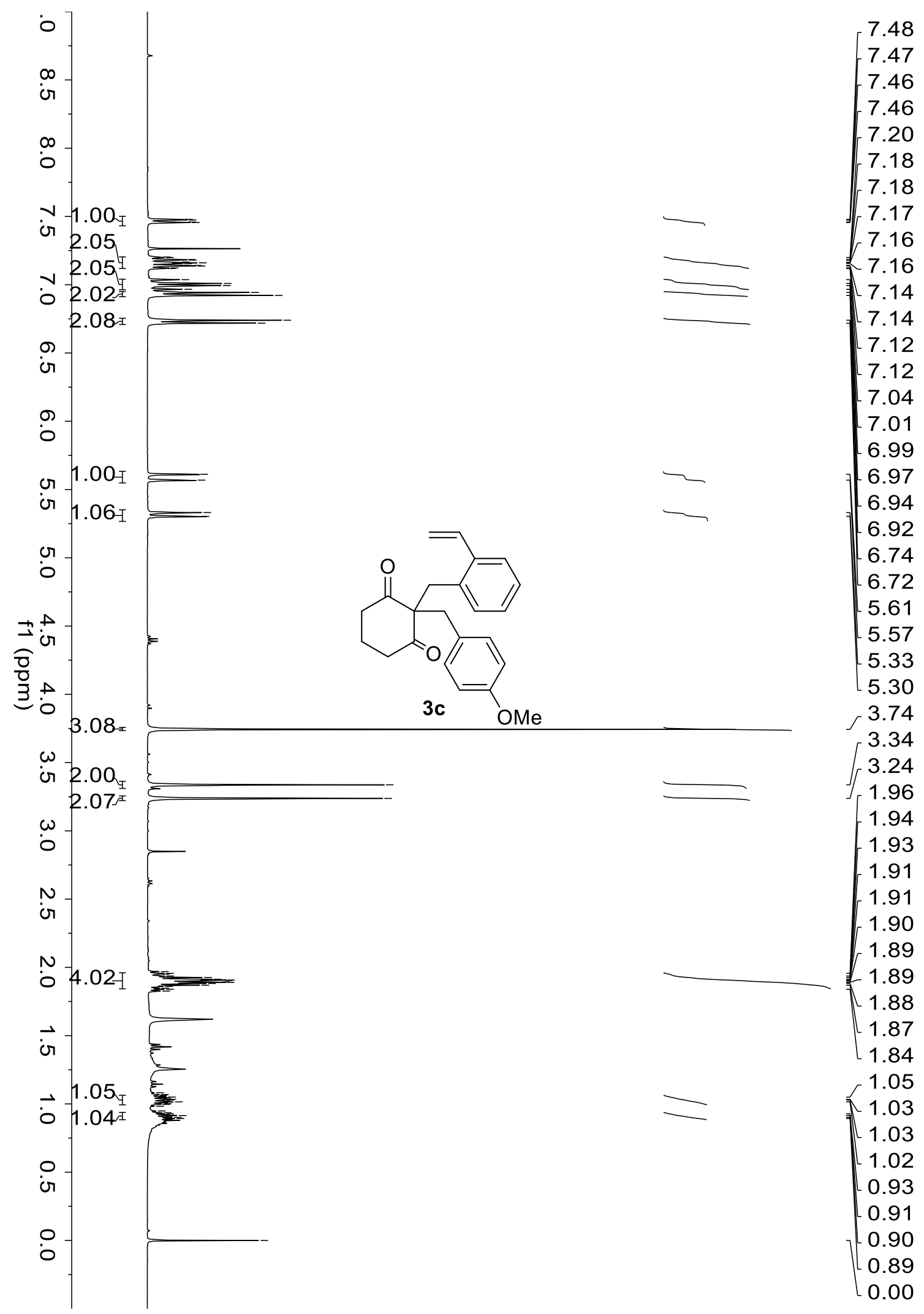




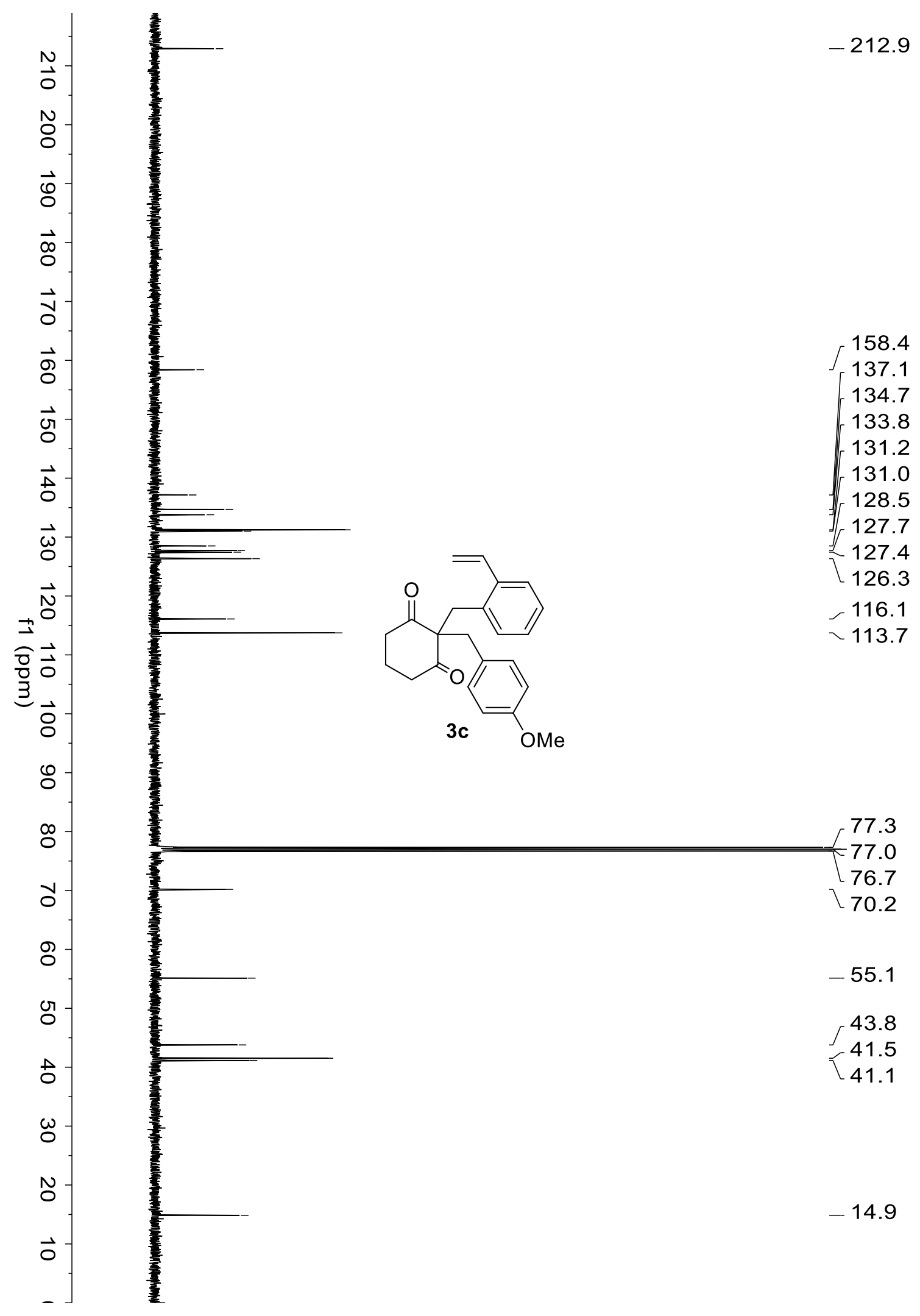




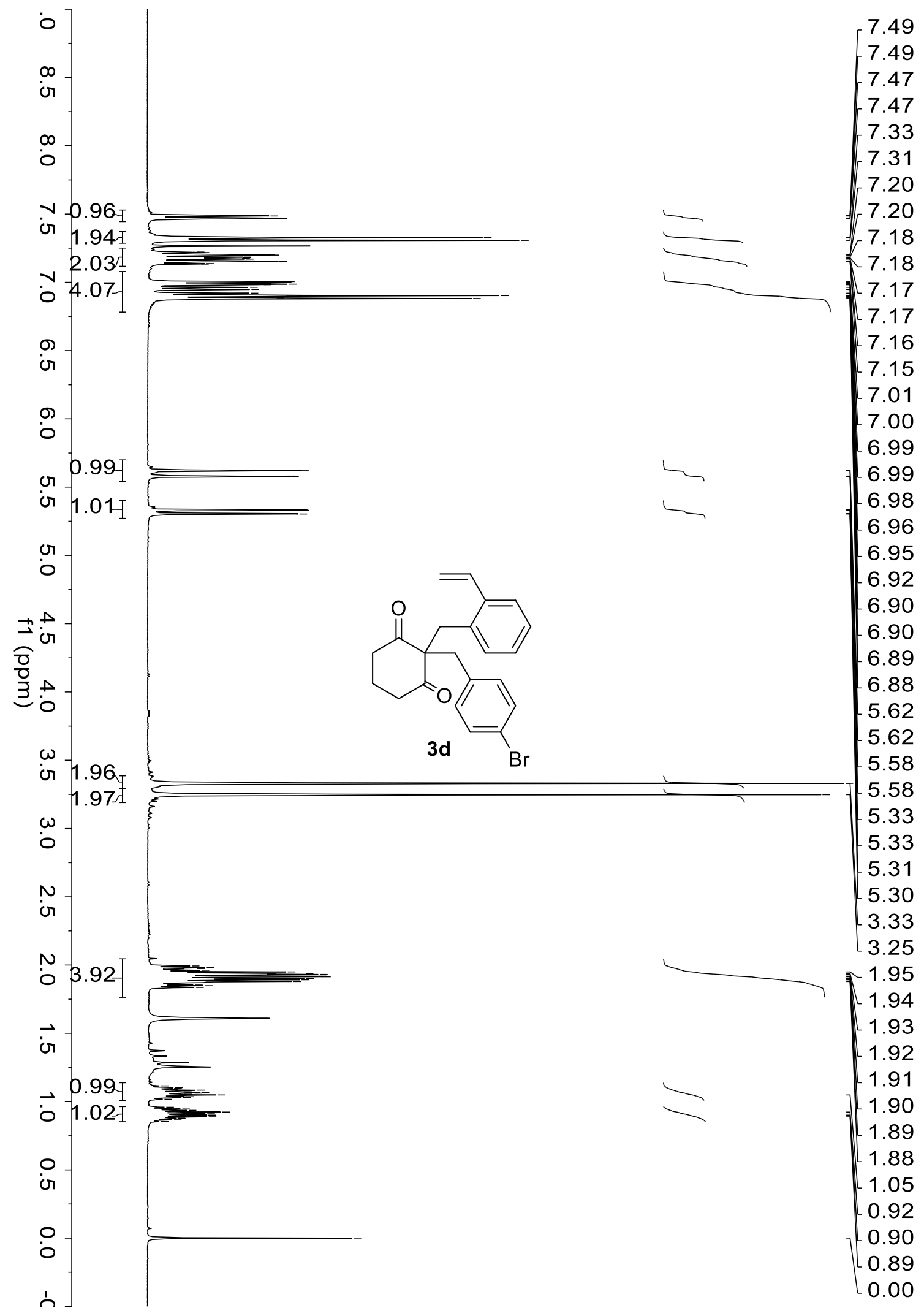




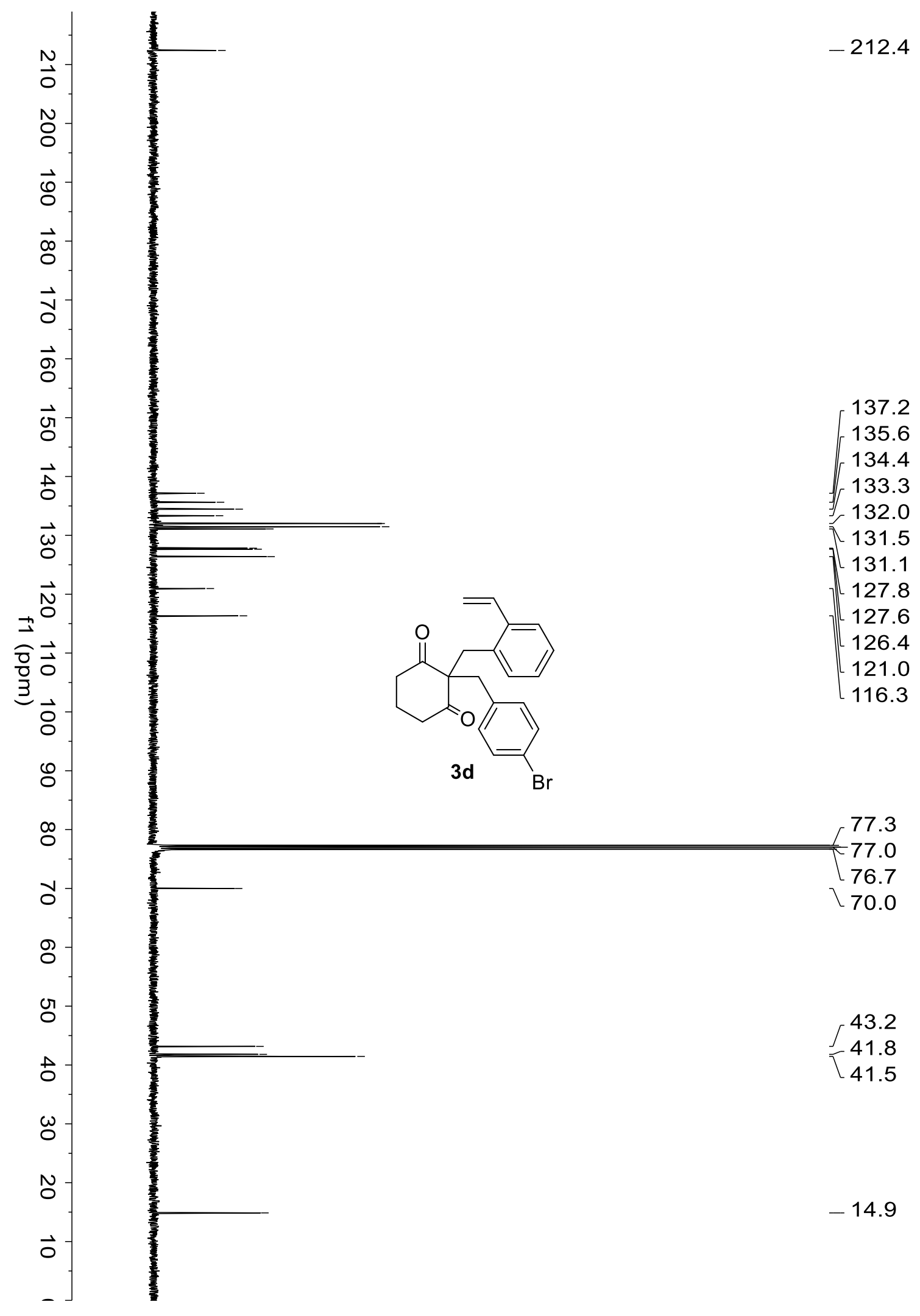




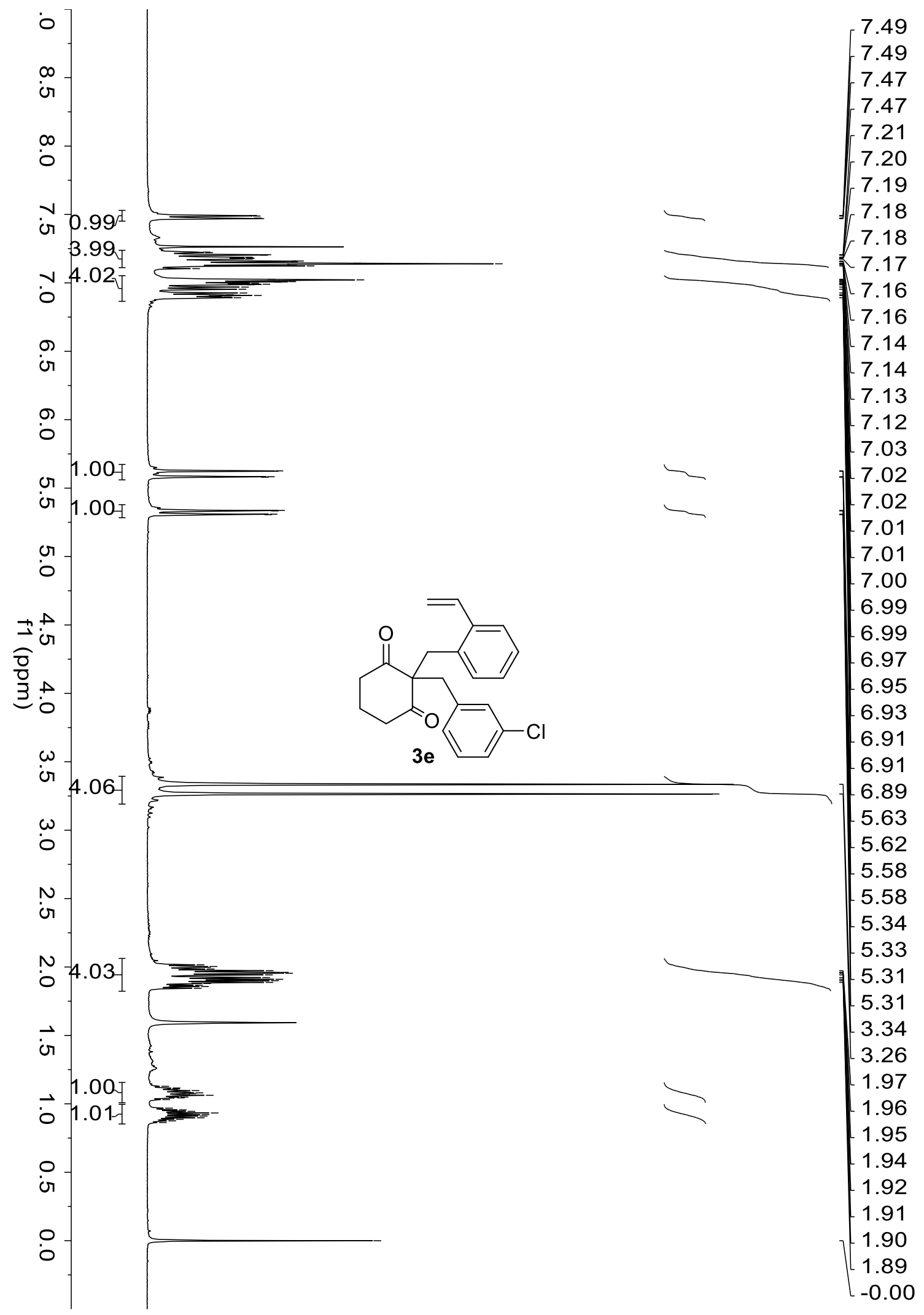




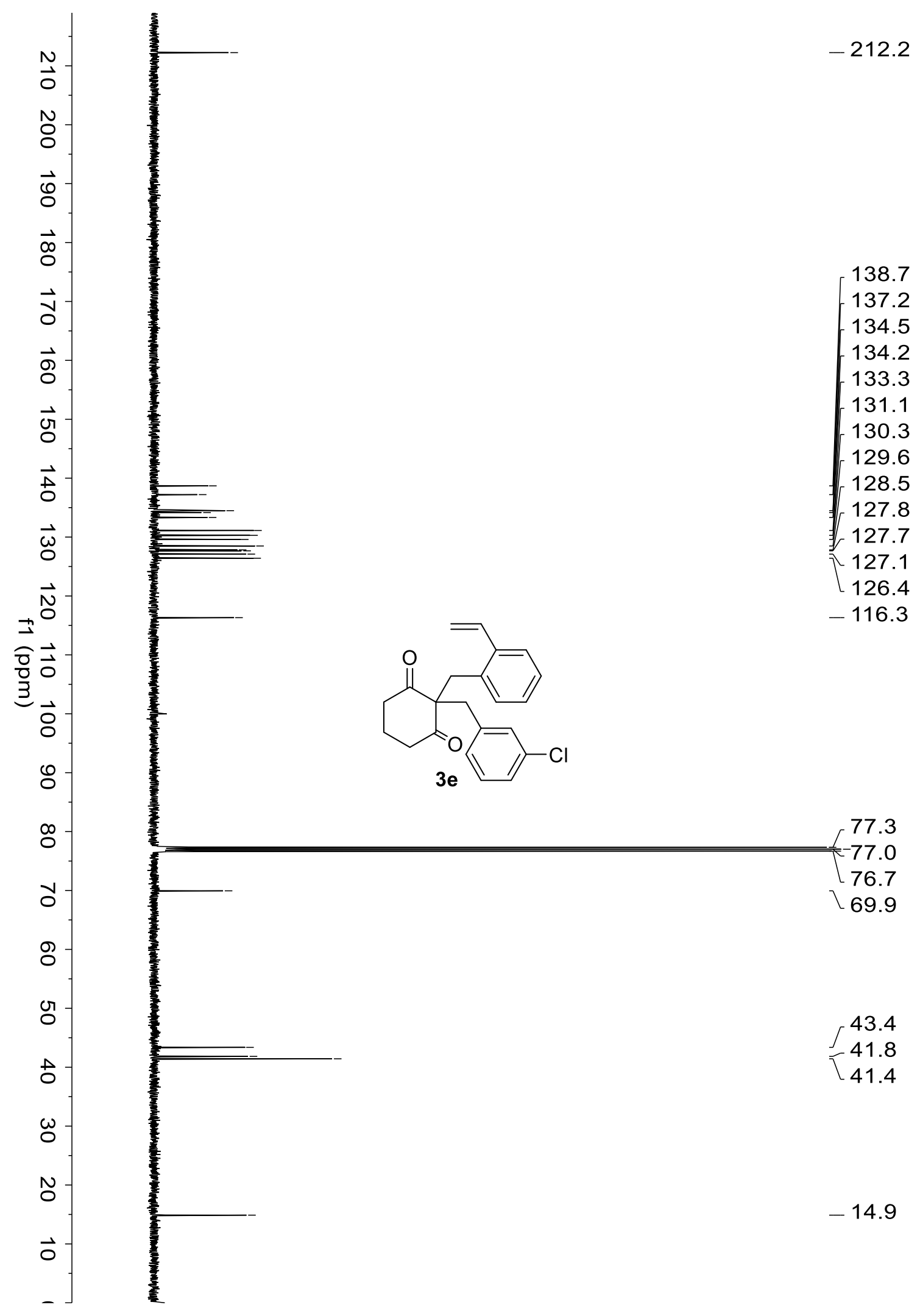




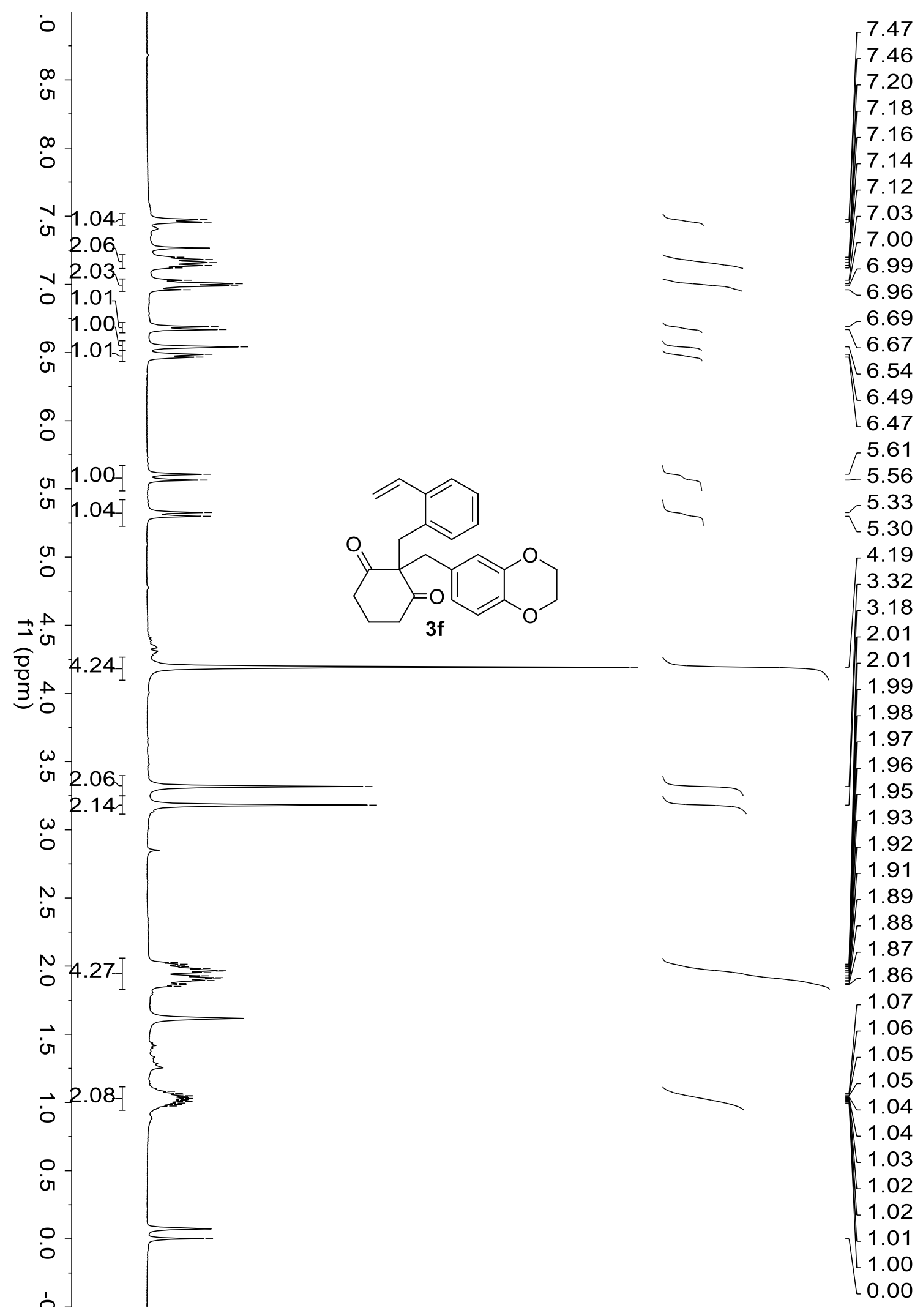




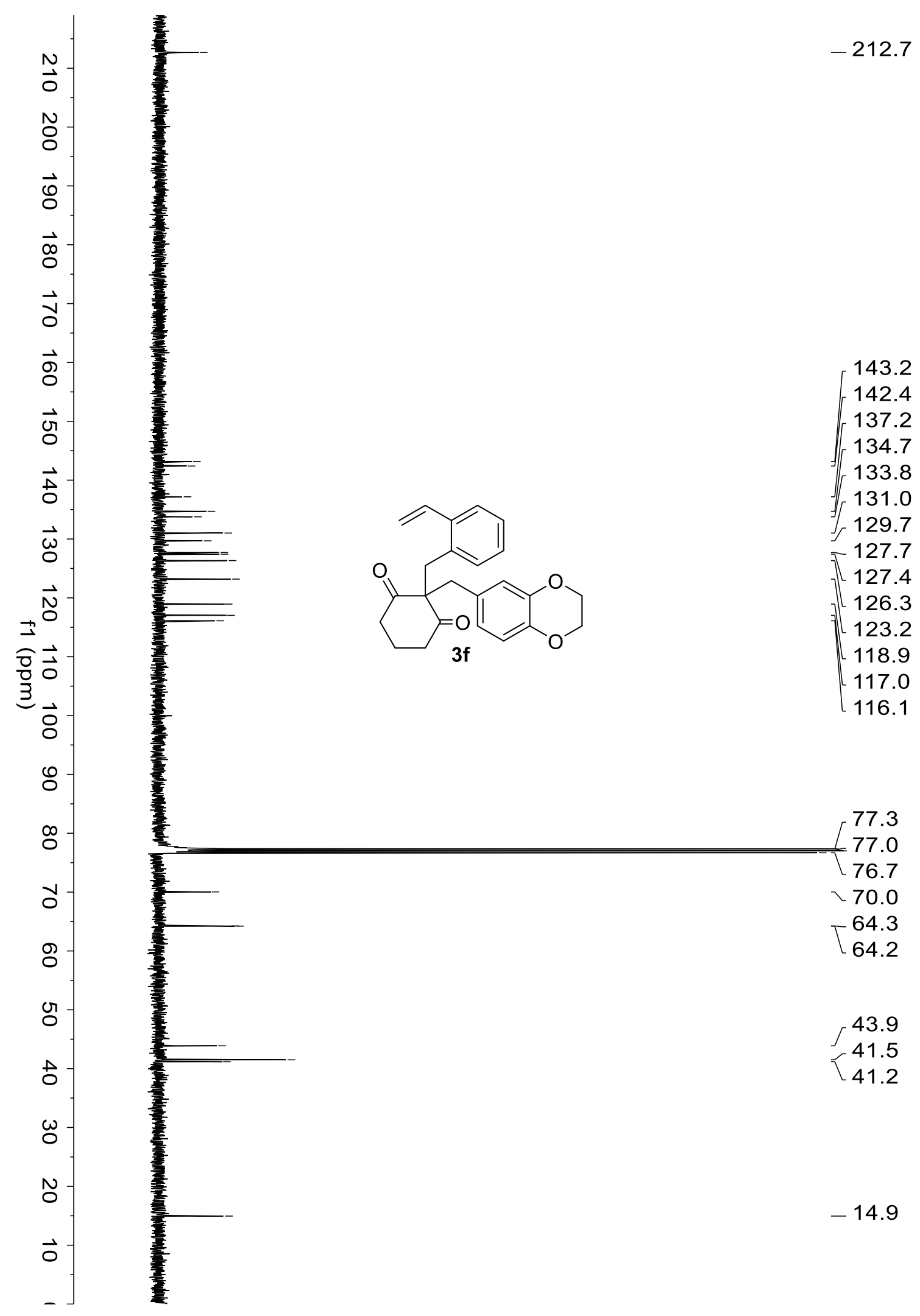




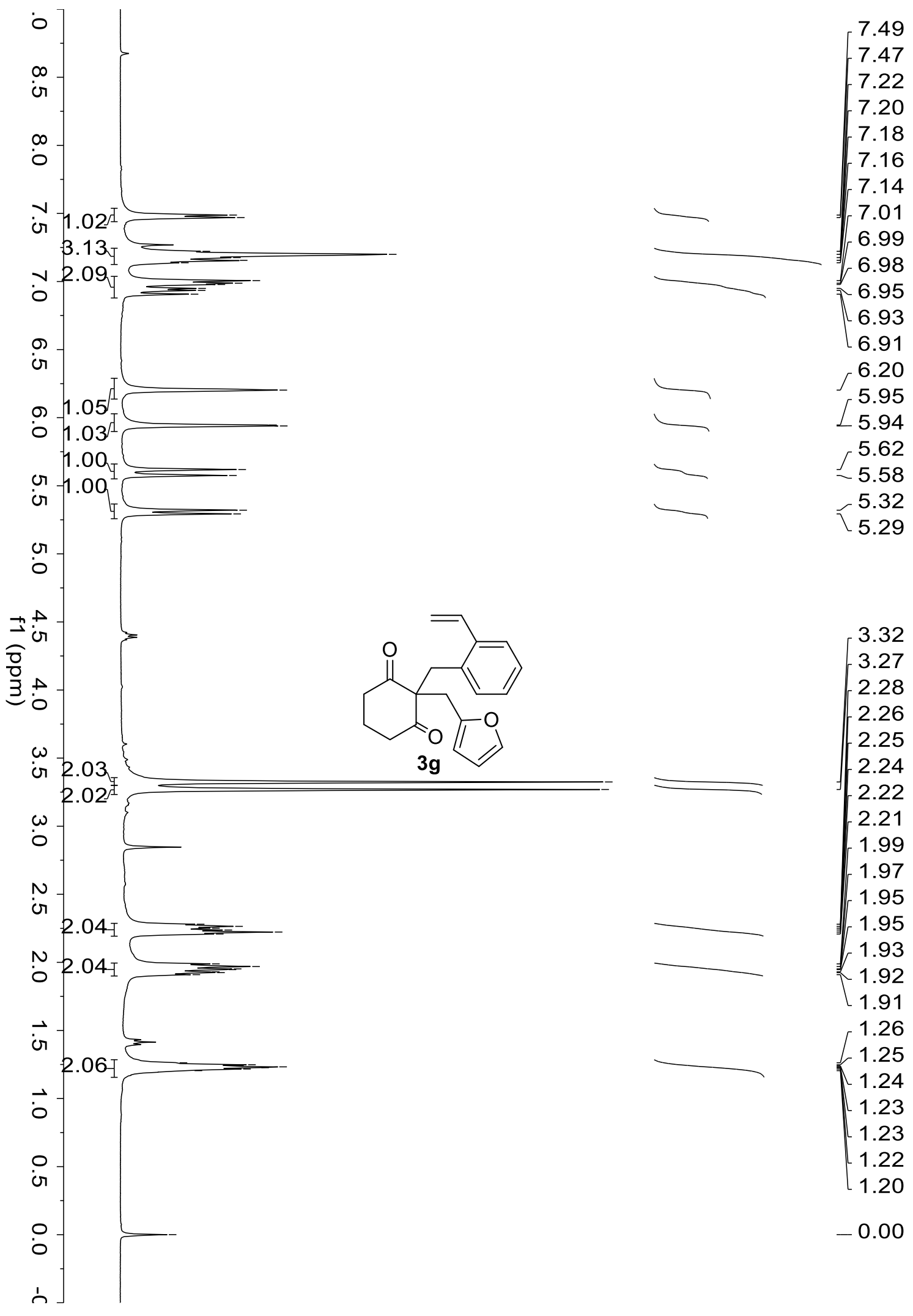




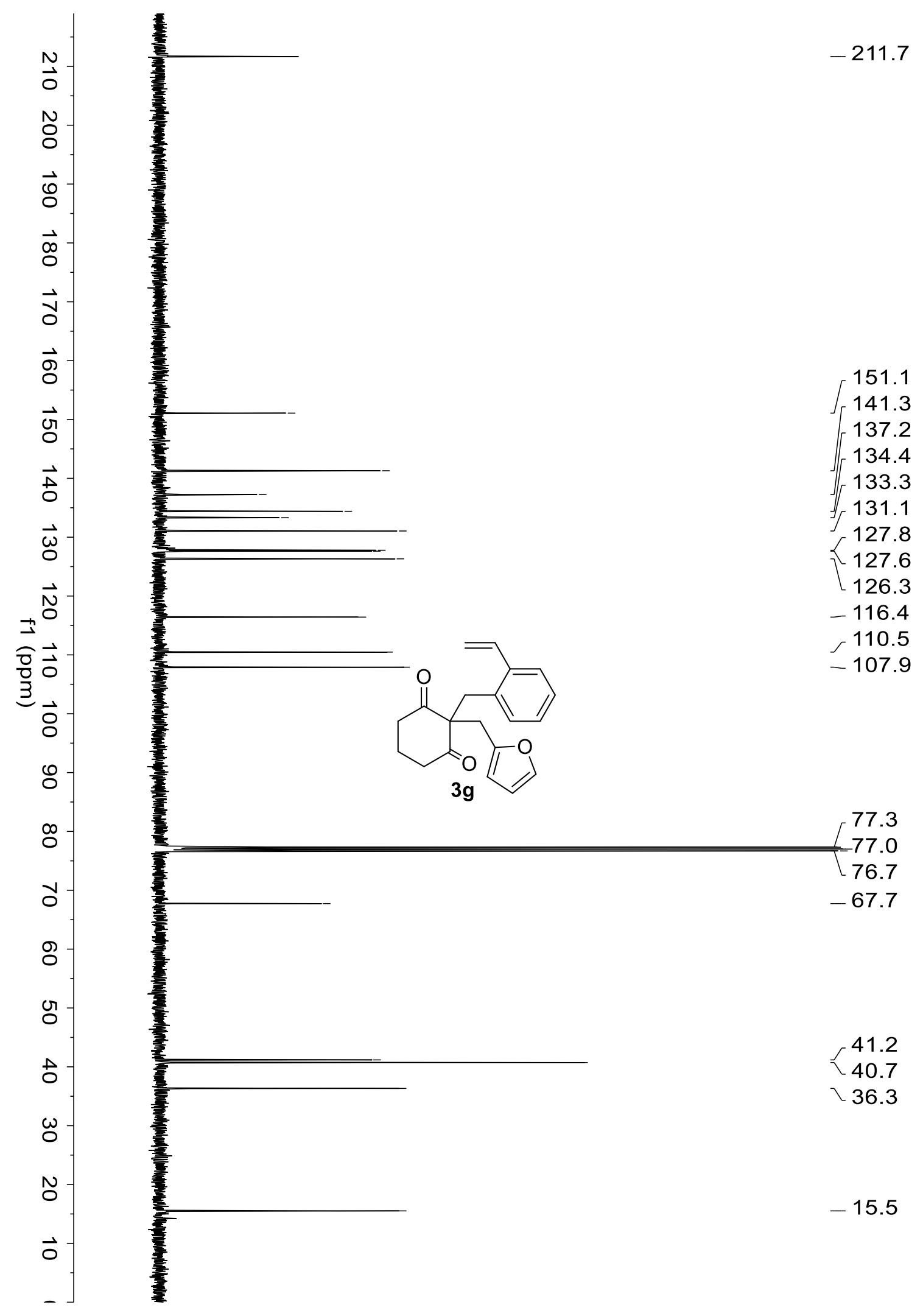




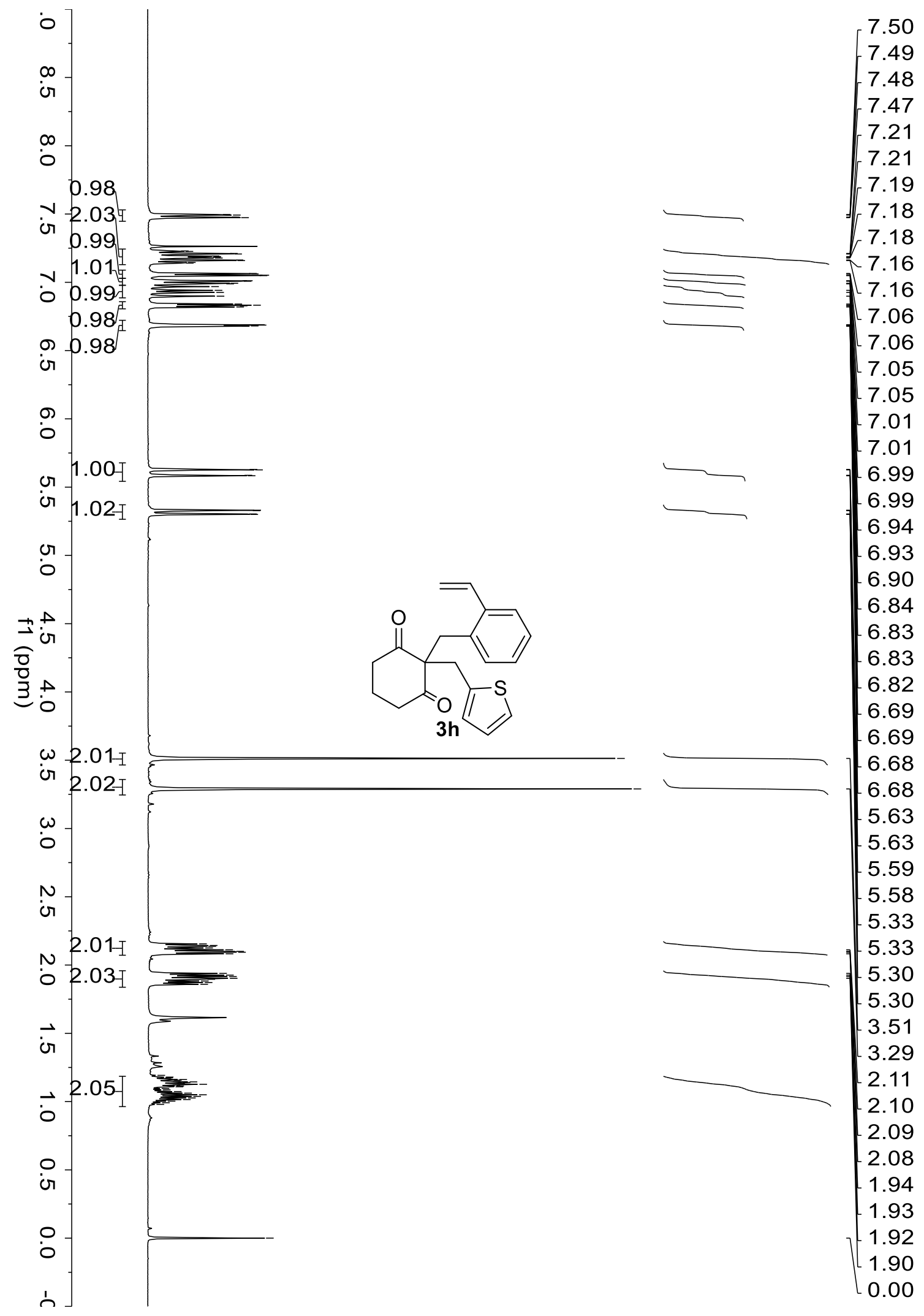




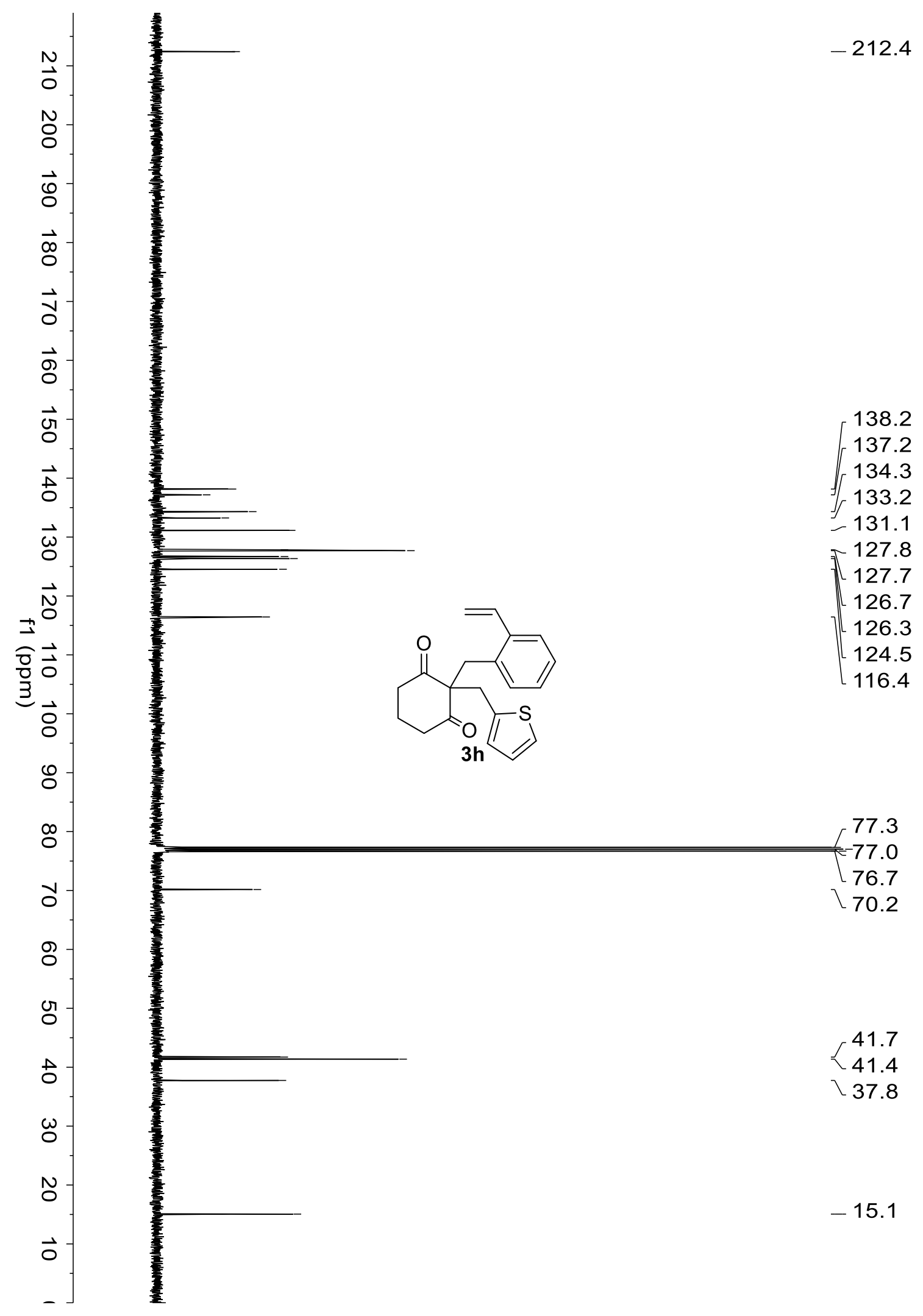




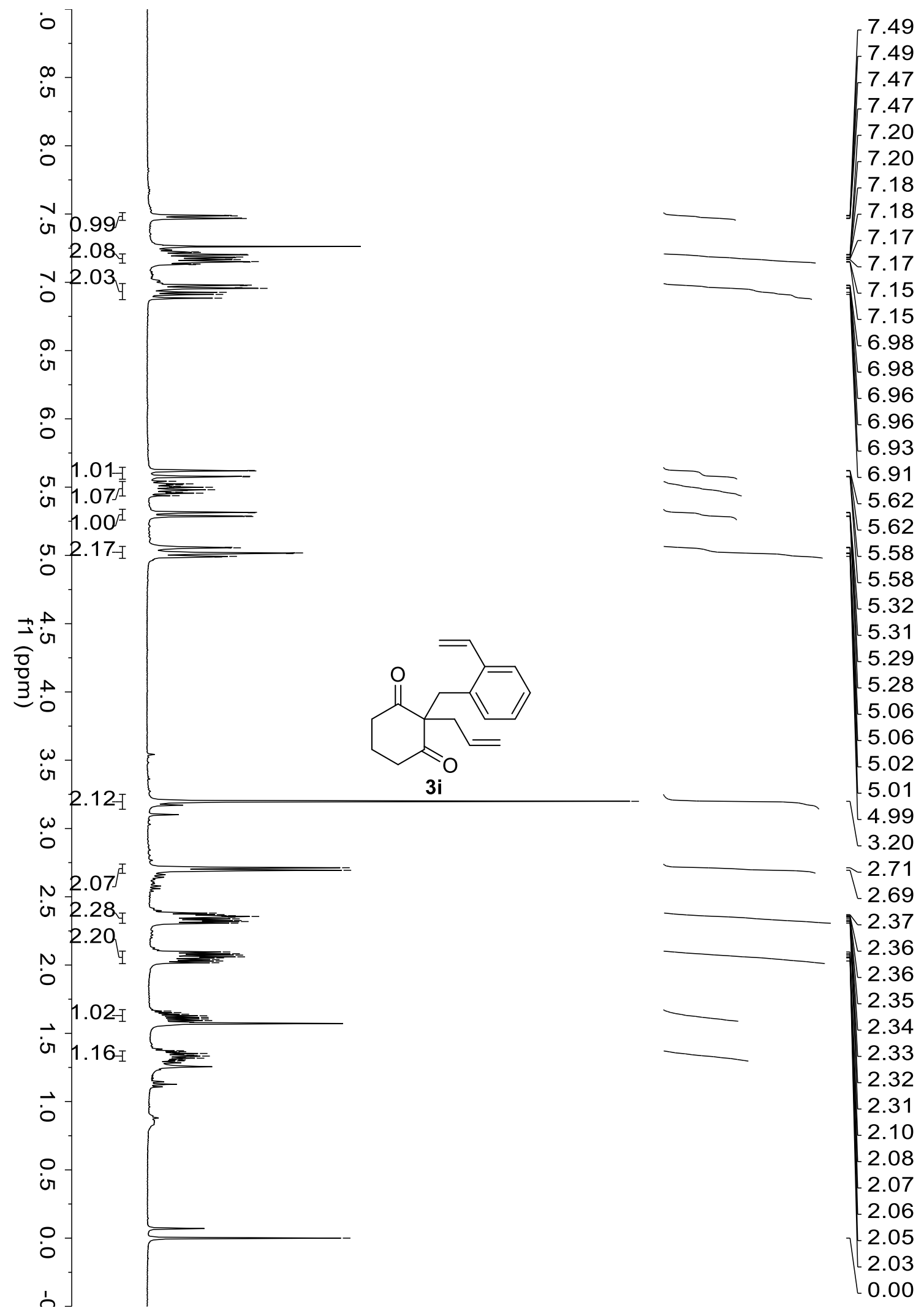




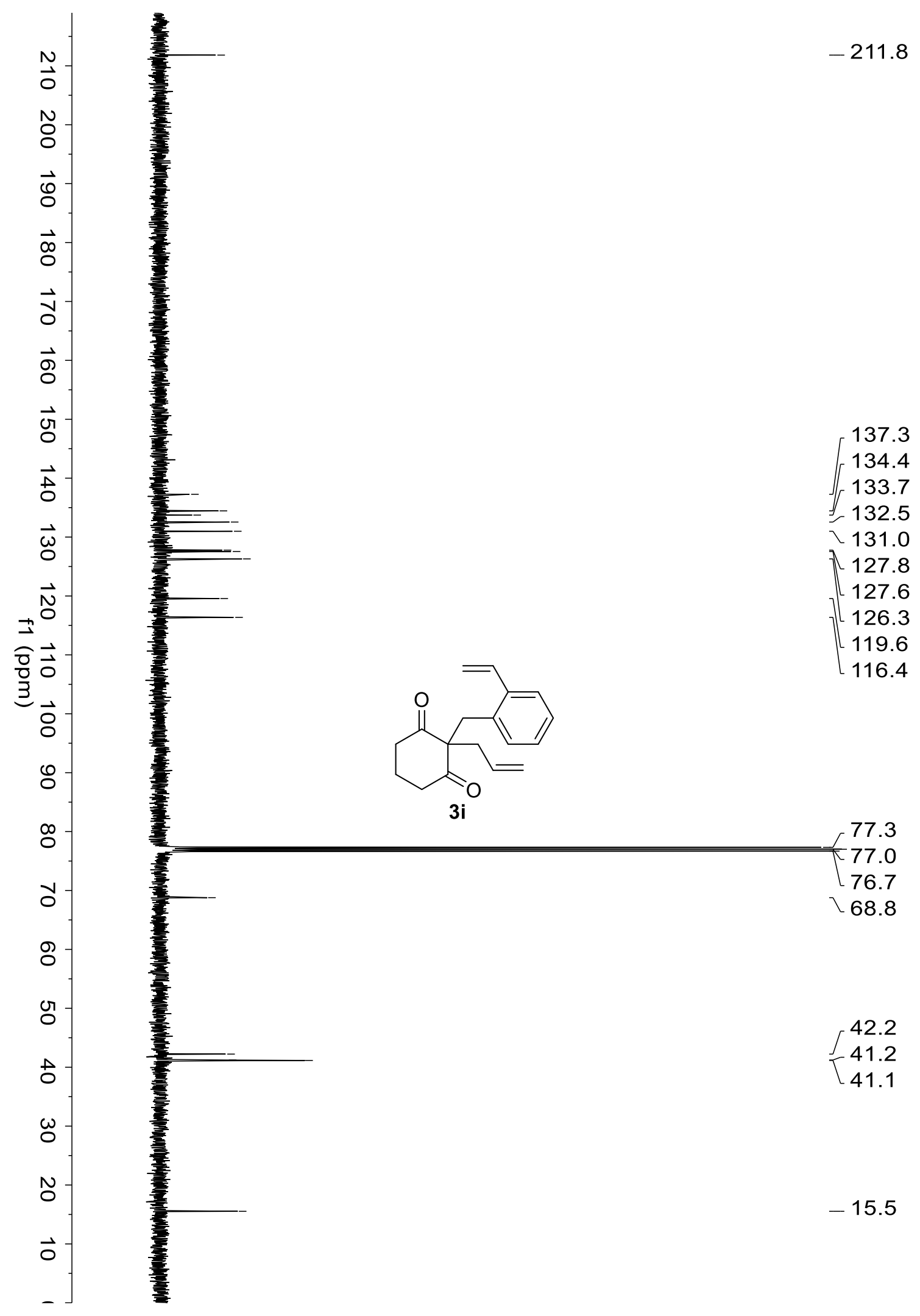



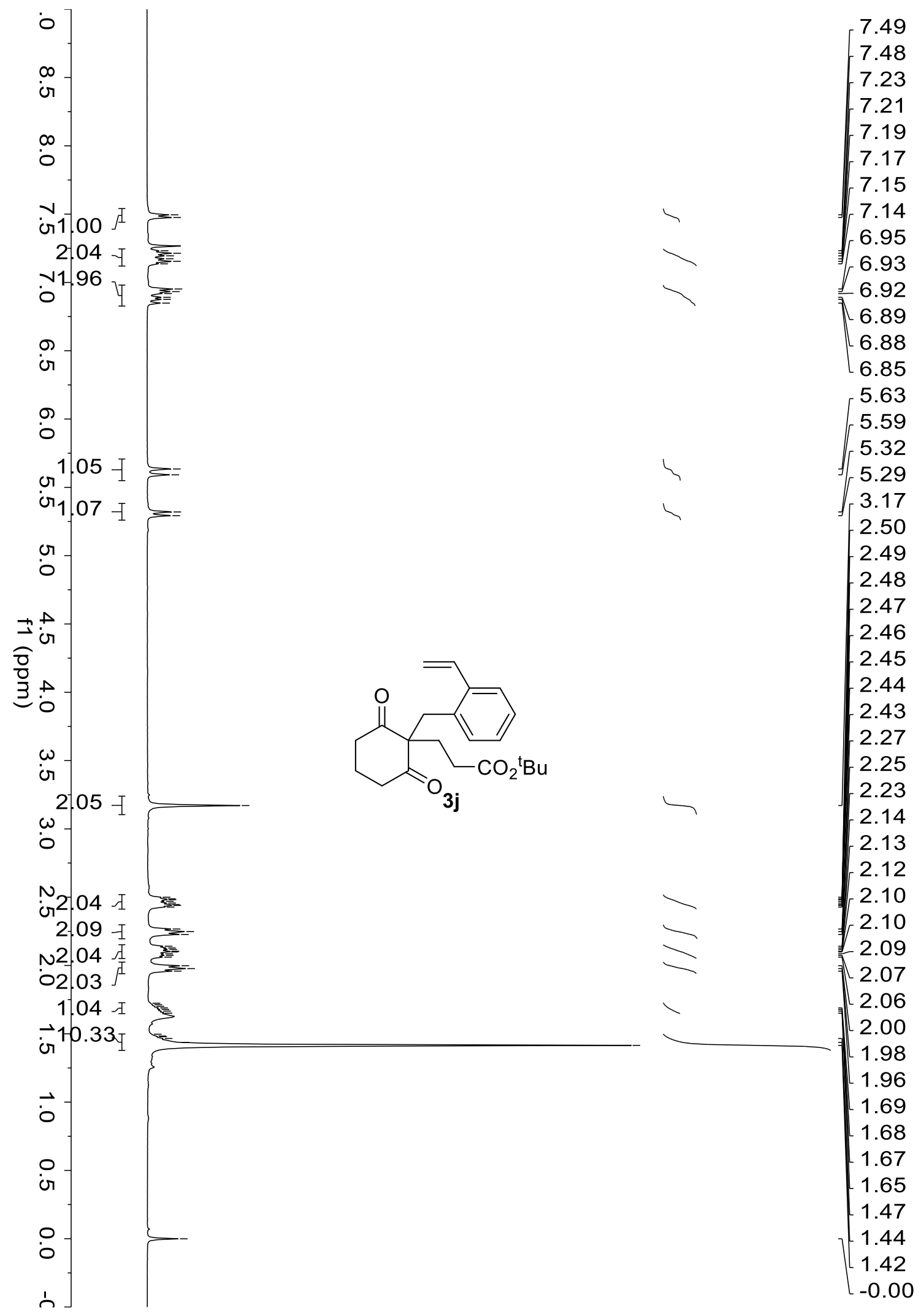


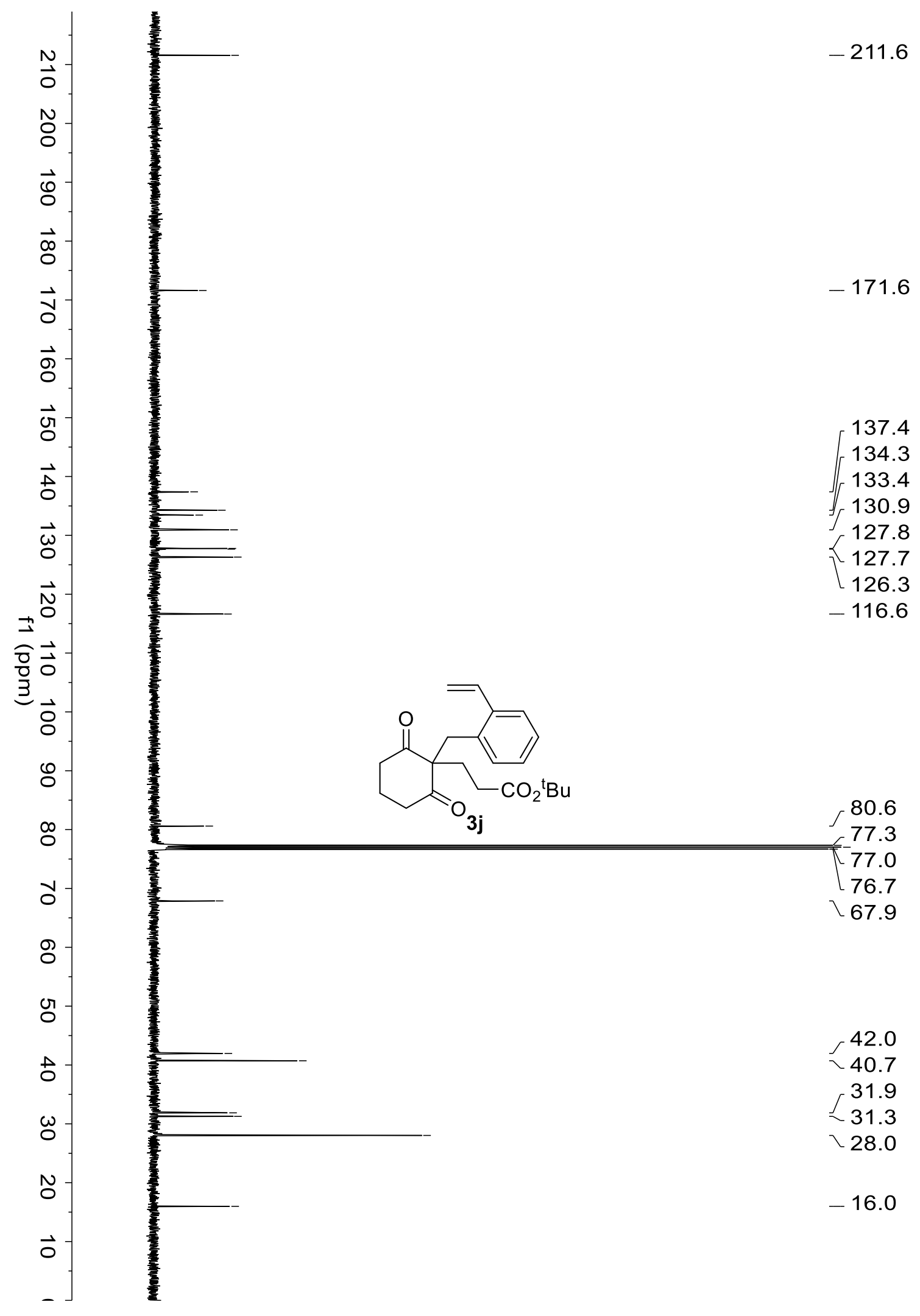




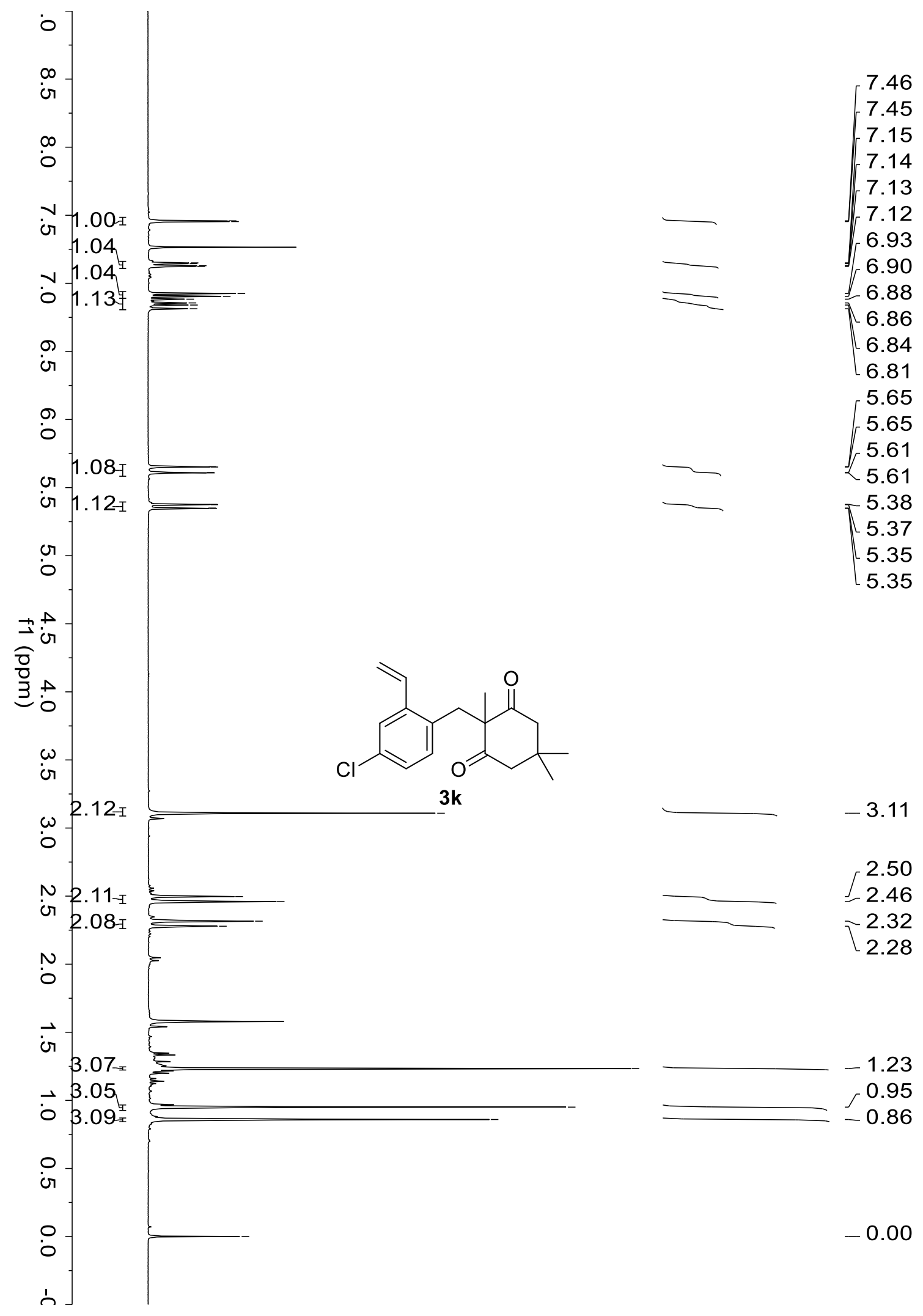




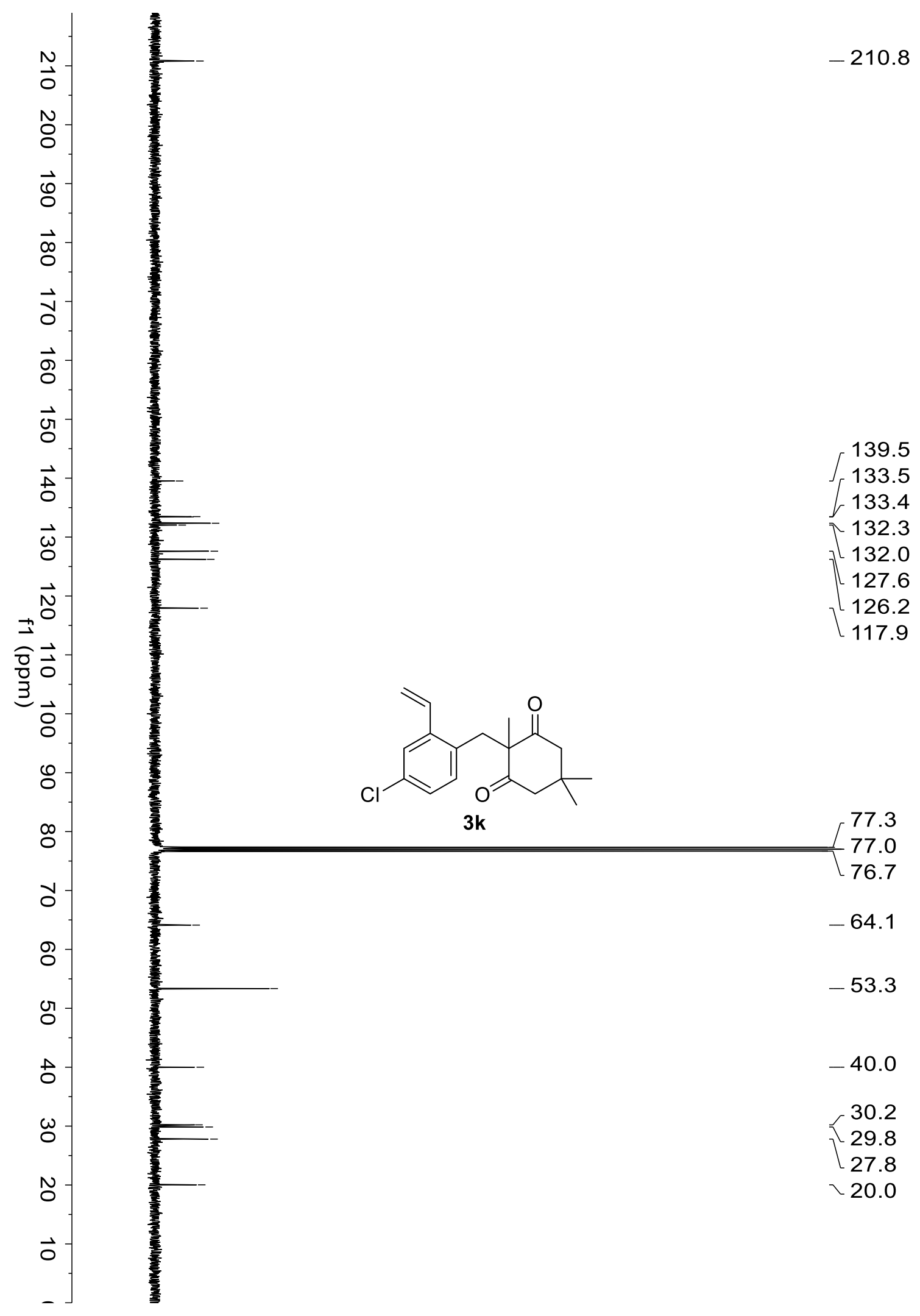




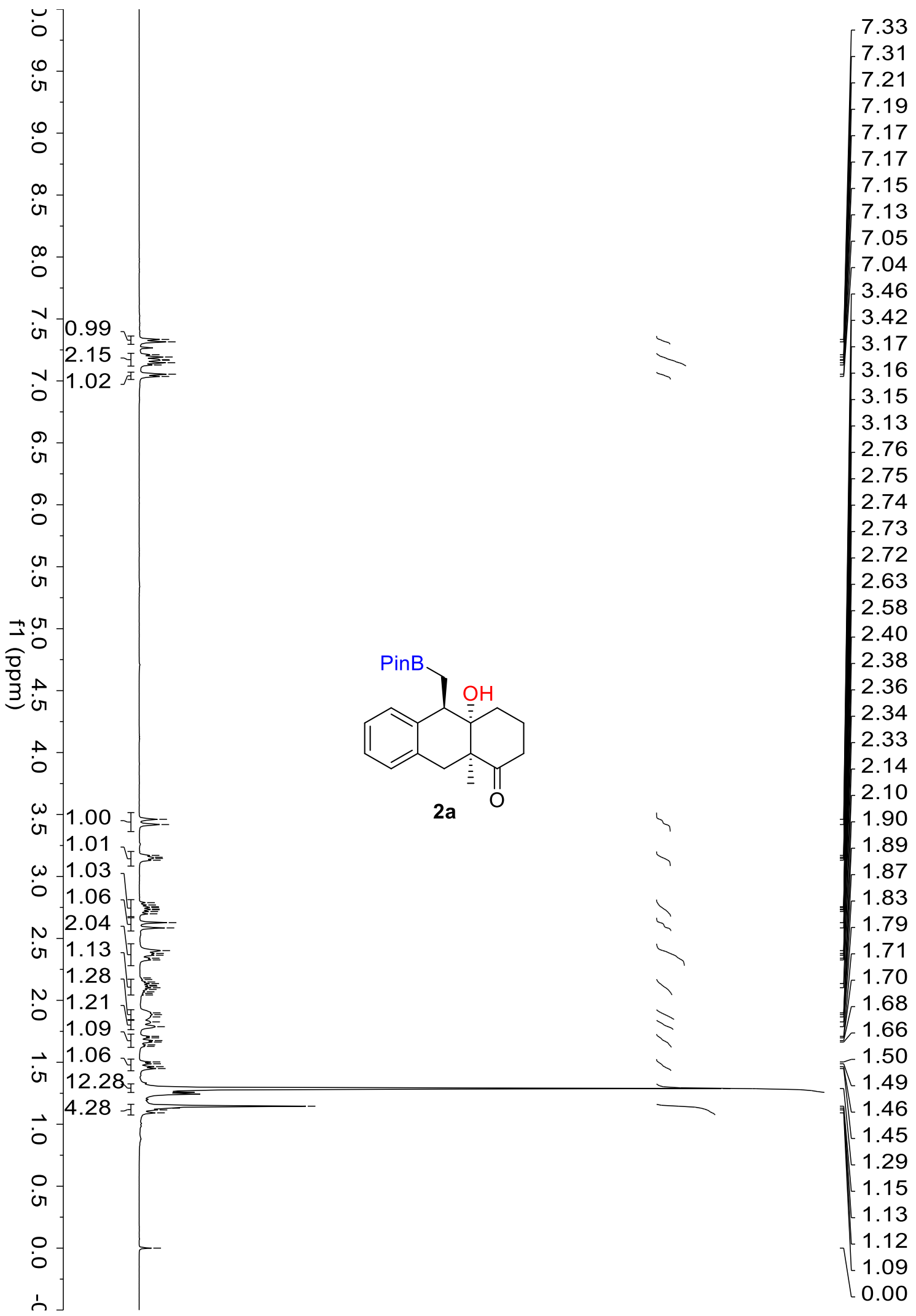




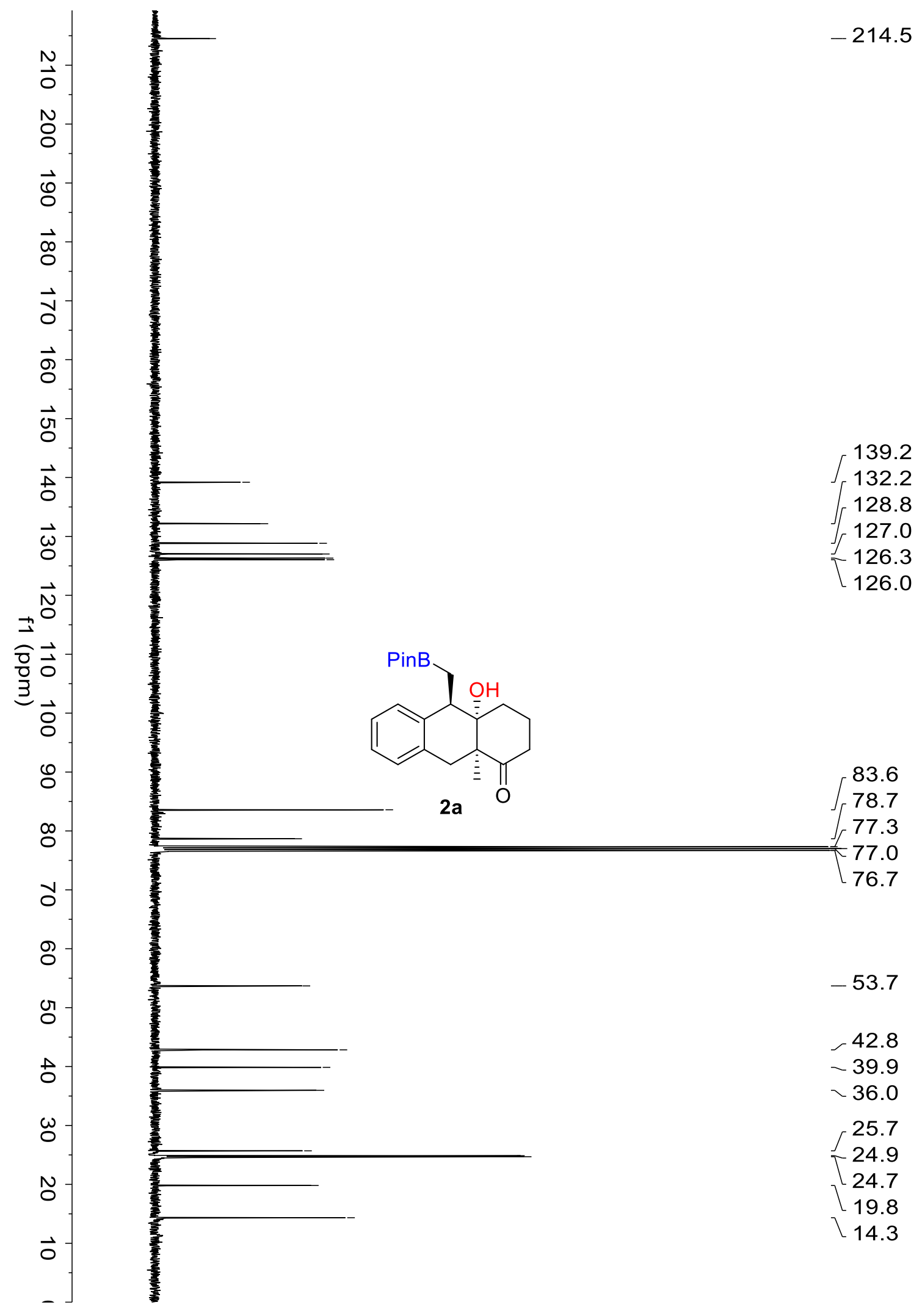




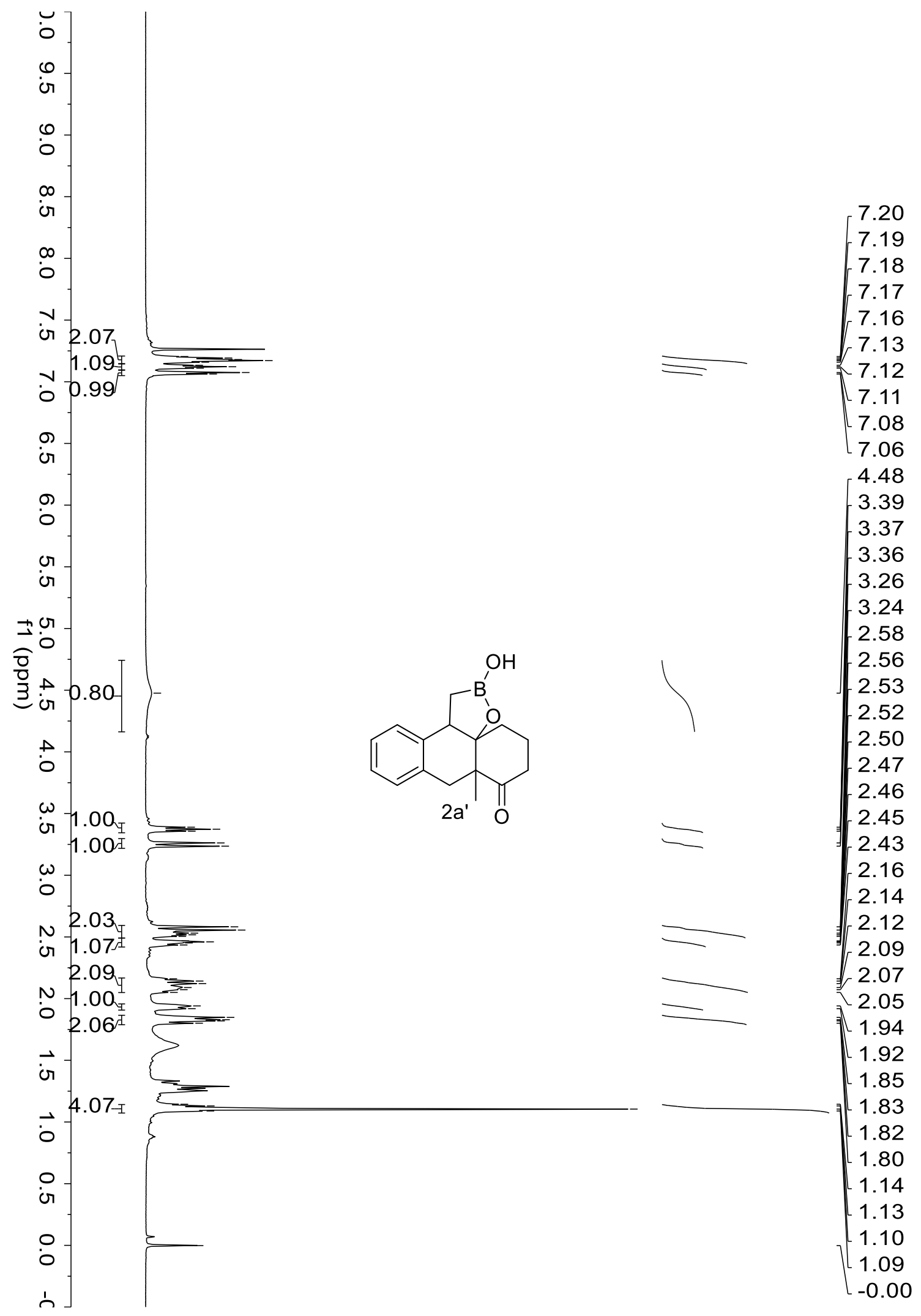




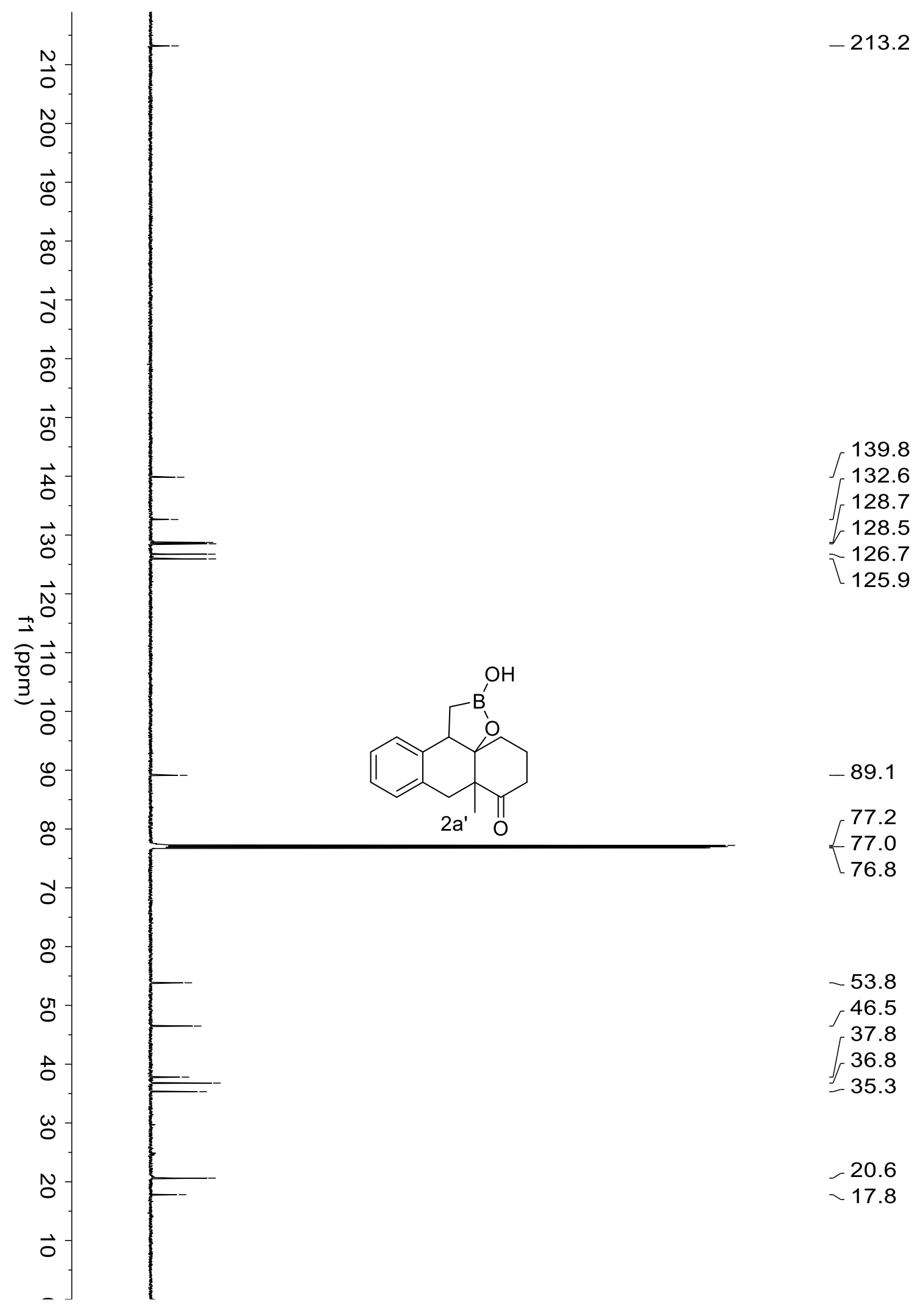




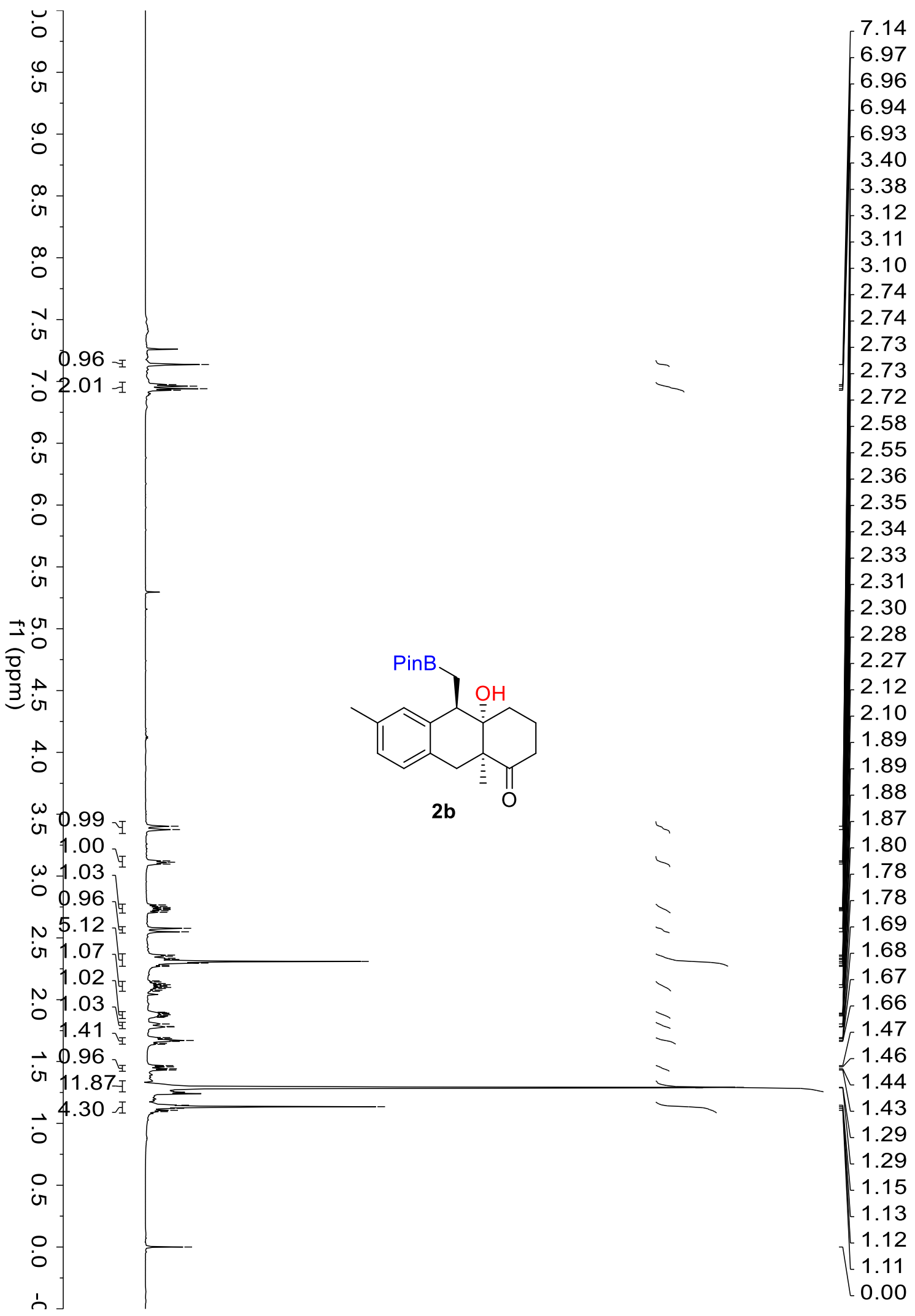




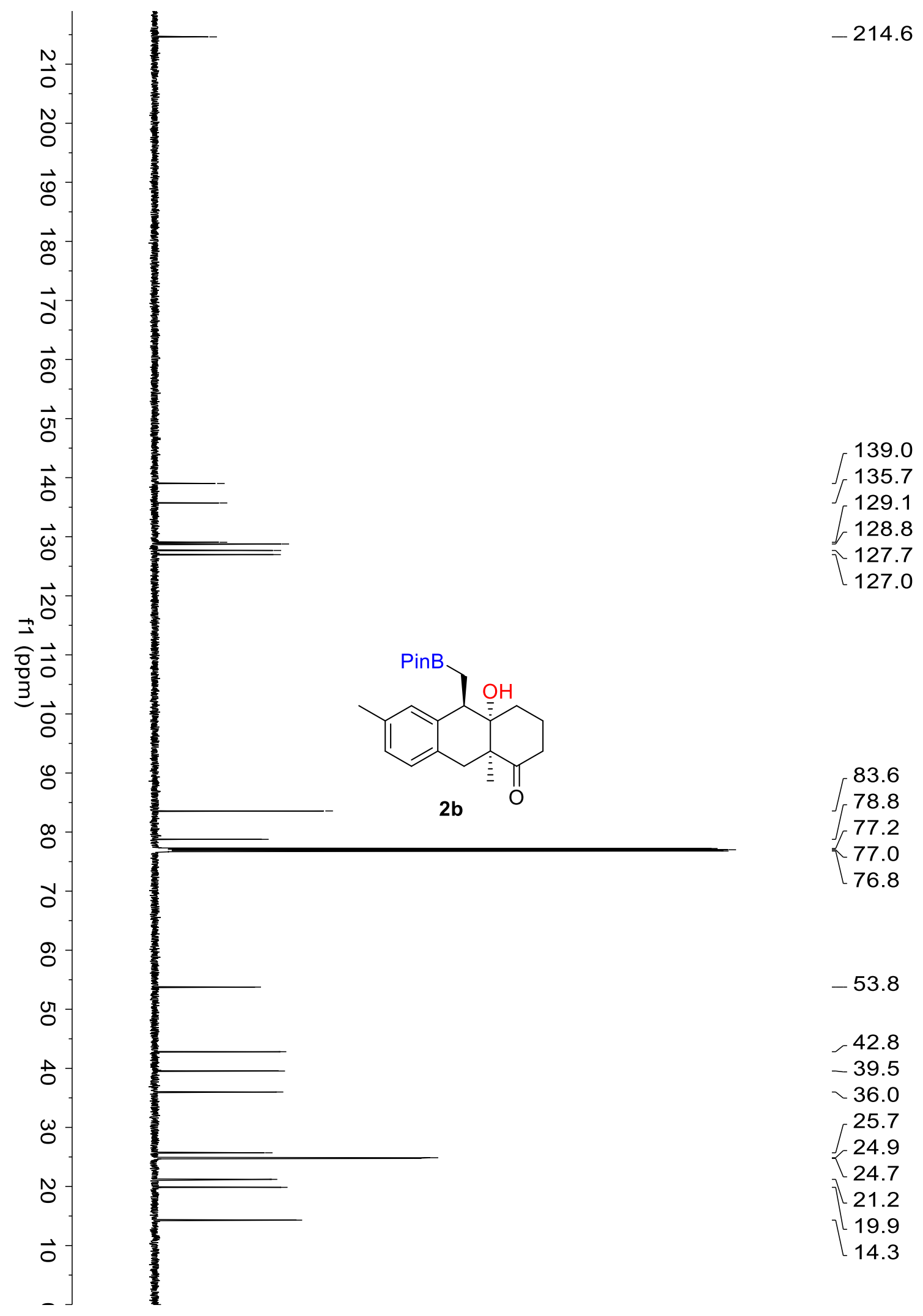




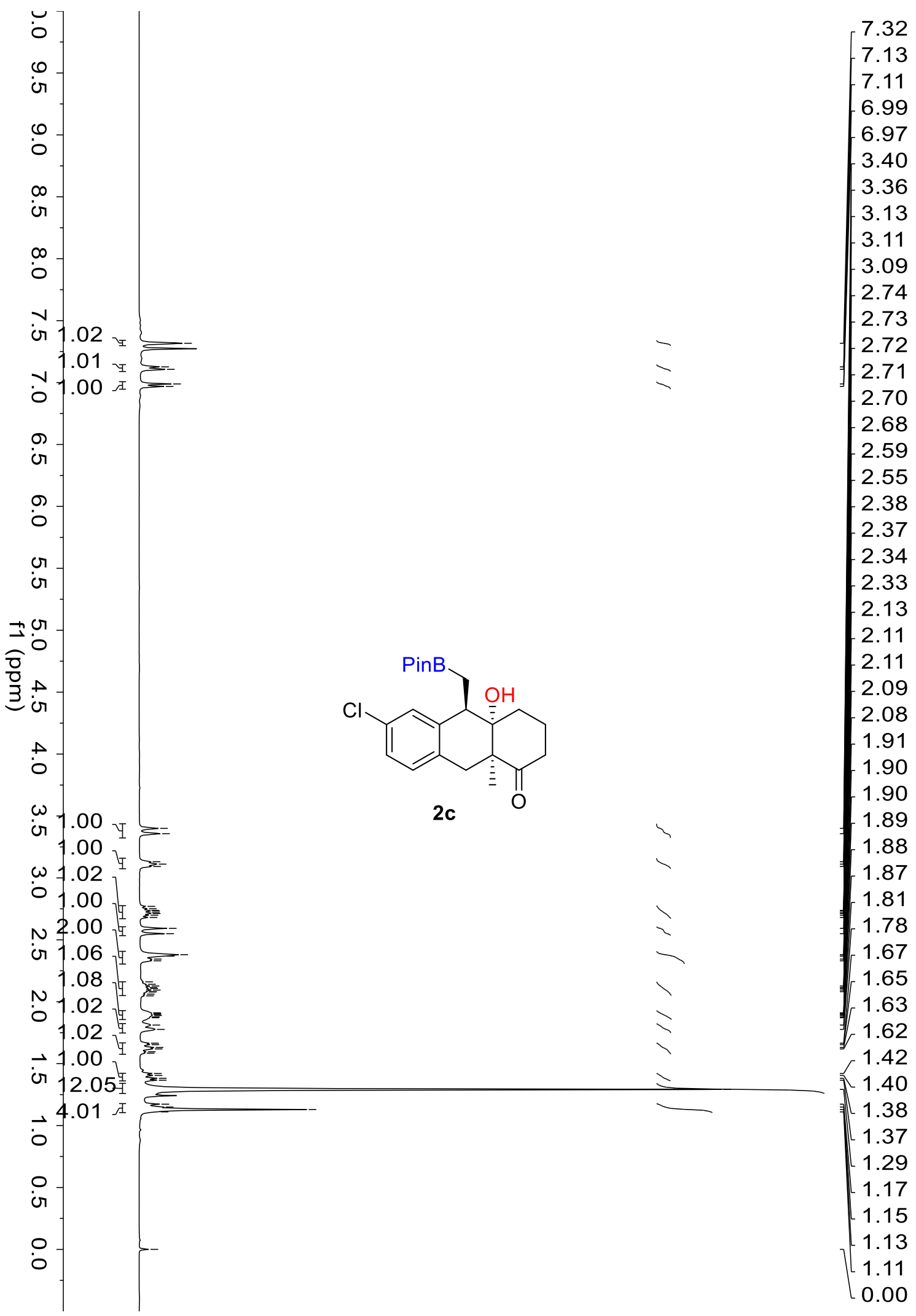




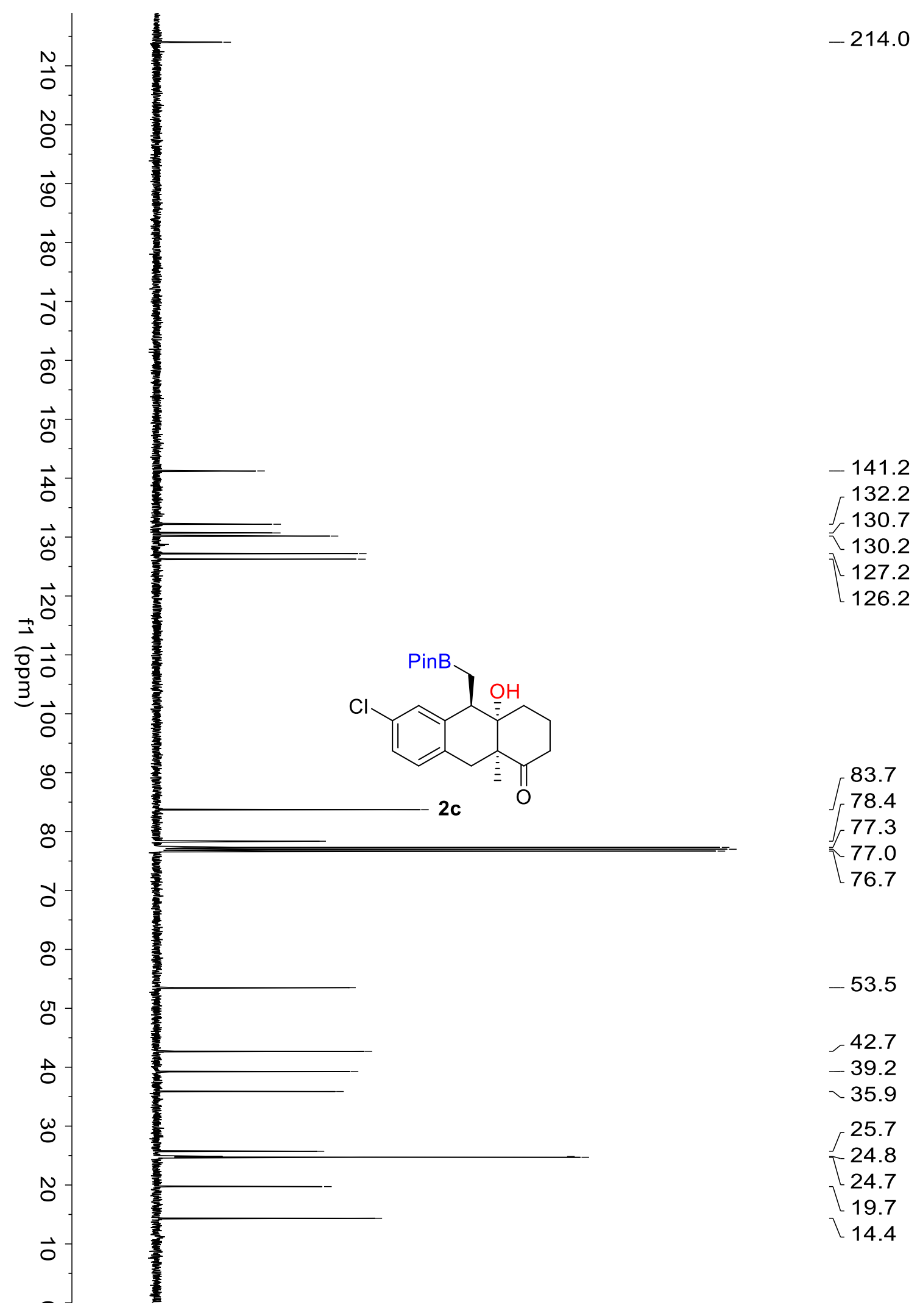




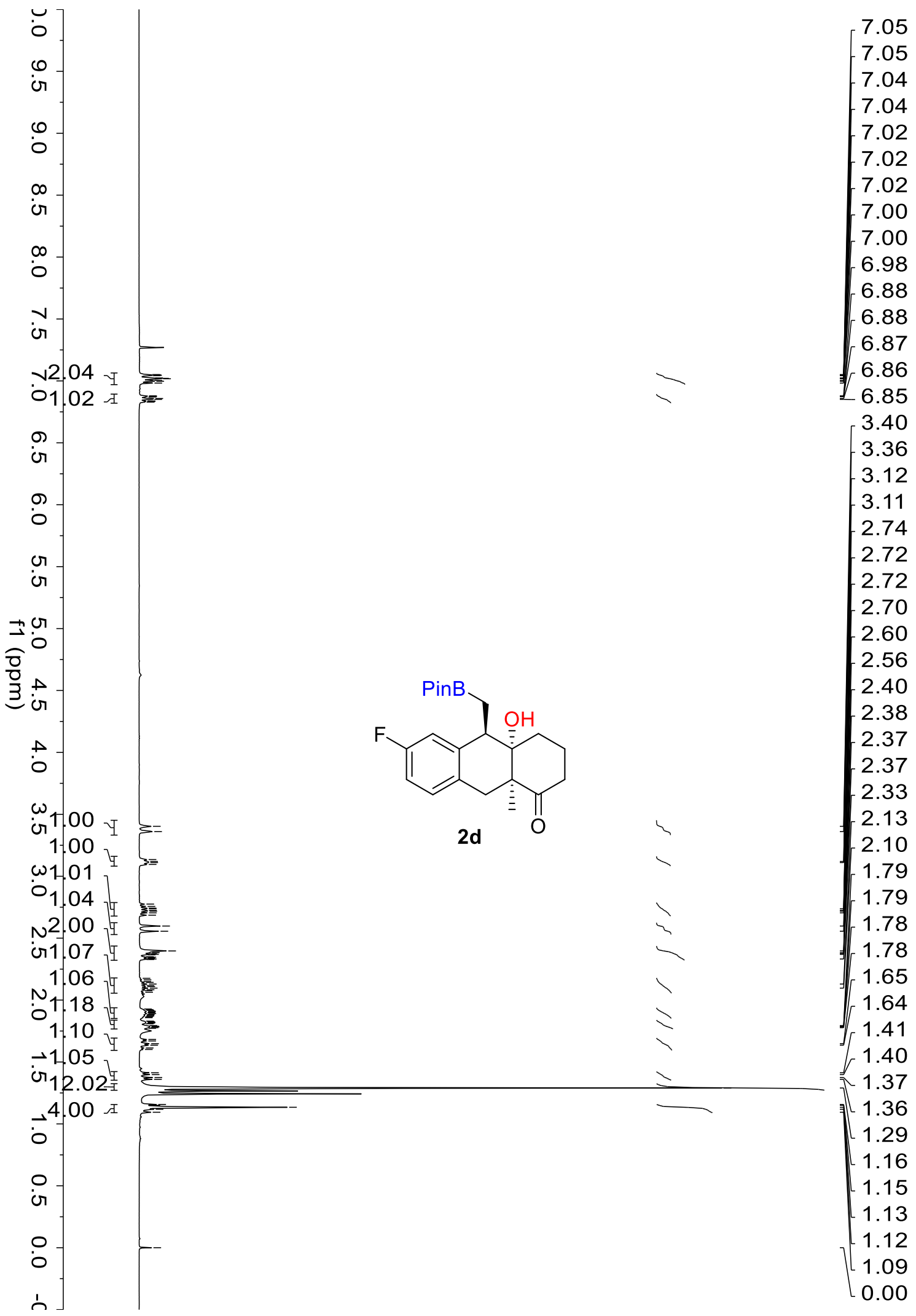




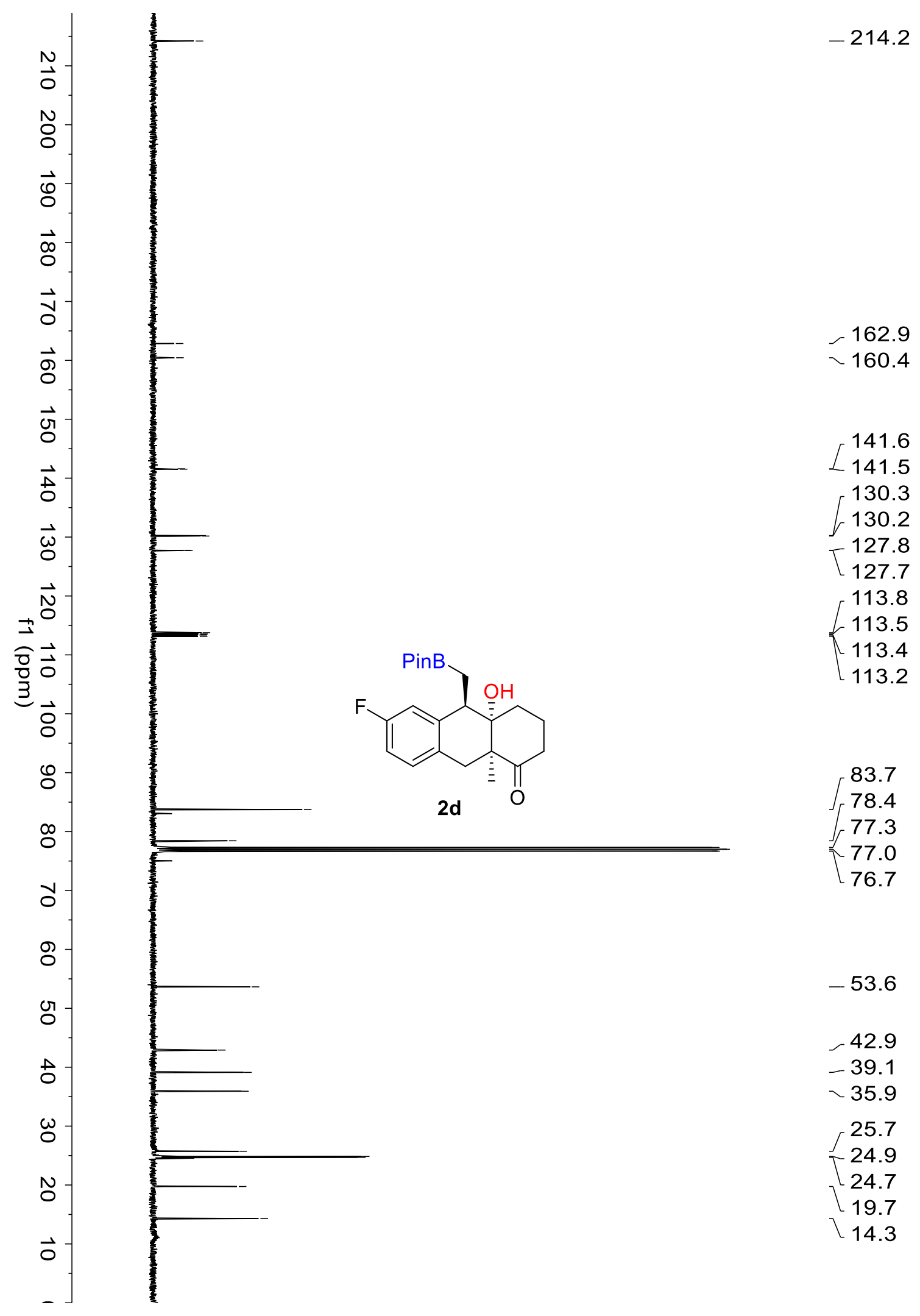




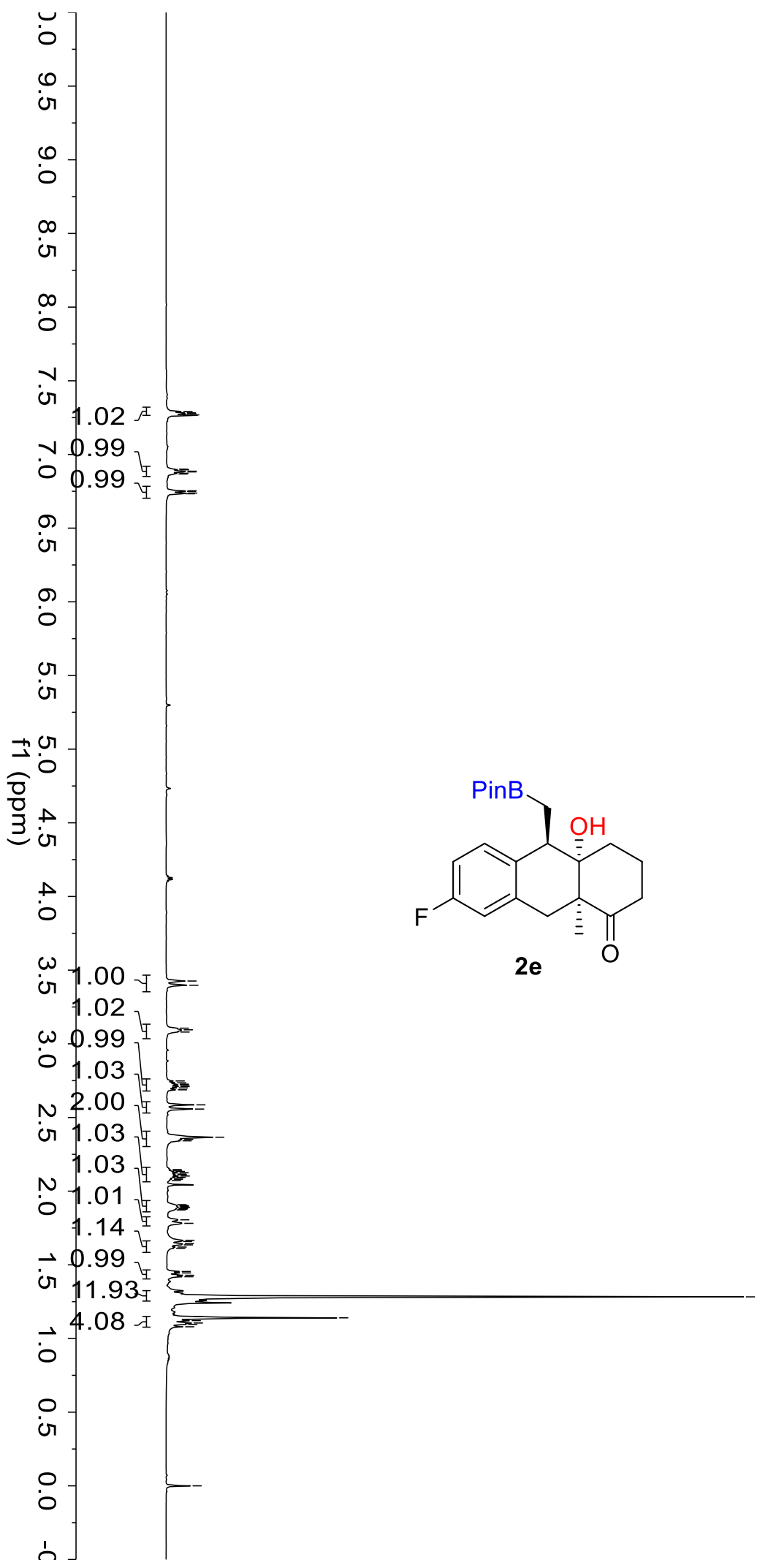

$\mid \begin{aligned} & 7.29 \\ & 7.28 \\ & 7.28 \\ & 7.27 \\ & 6.90 \\ & 6.89 \\ & 6.88 \\ & 6.87 \\ & 6.75 \\ & 6.75 \\ & 6.74 \\ & 6.73 \\ & 3.43 \\ & 3.40 \\ & 3.11 \\ & 3.10 \\ & 3.08 \\ & 2.73 \\ & 2.72 \\ & 2.71 \\ & 2.71 \\ & 2.59 \\ & 2.56 \\ & 2.37 \\ & 2.35 \\ & 2.34 \\ & 2.12 \\ & 2.10 \\ & 1.90 \\ & 1.89 \\ & 1.89 \\ & 1.81 \\ & 1.78 \\ & 1.67 \\ & 1.66 \\ & 1.64 \\ & 1.64 \\ & 1.45 \\ & 1.44 \\ & 1.43 \\ & 1.42 \\ & 1.28 \\ & 1.15 \\ & 1.14 \\ & 1.12 \\ & 1.11 \\ & 1.10 \\ & 1.08 \\ & 0.00\end{aligned}$




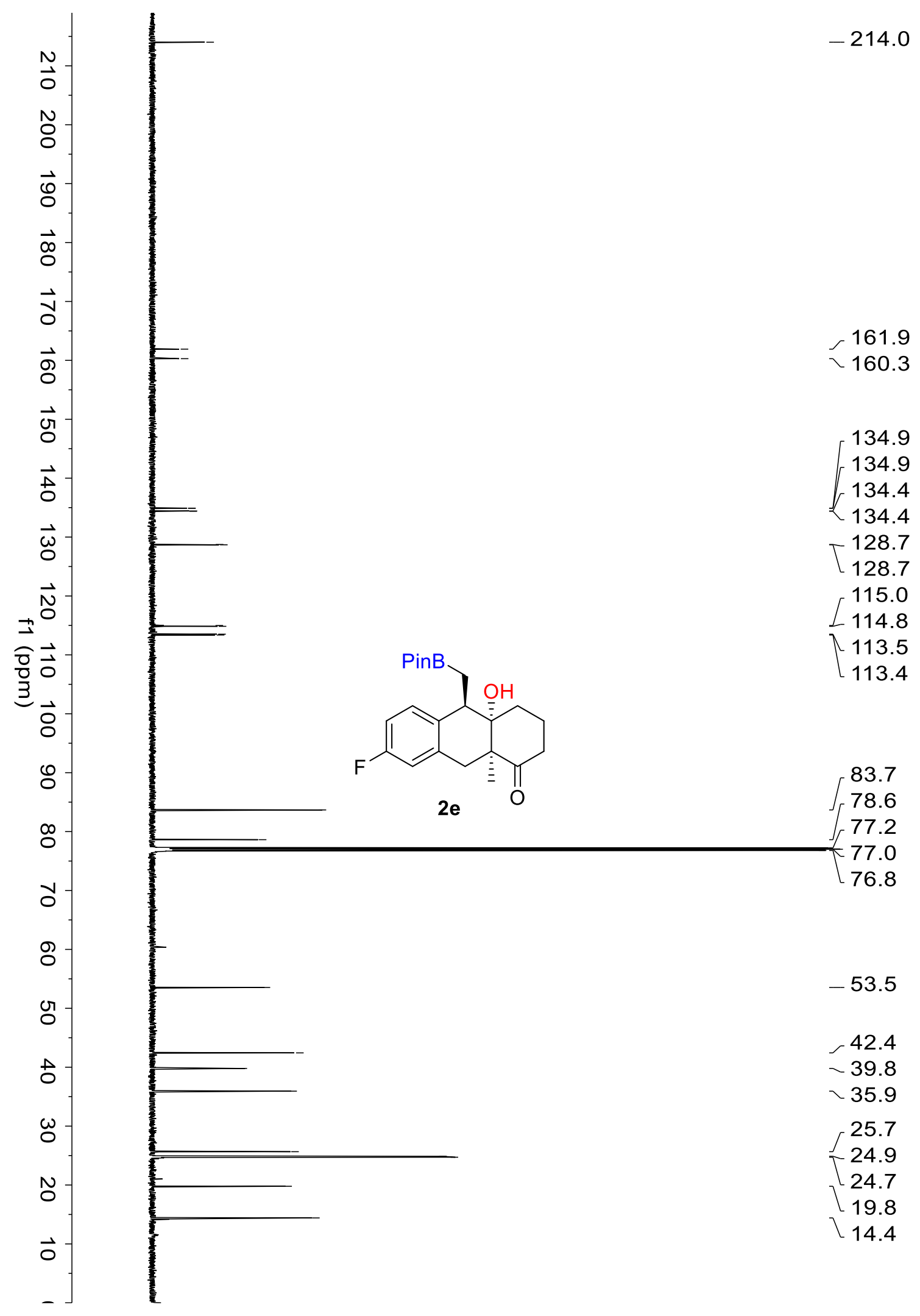




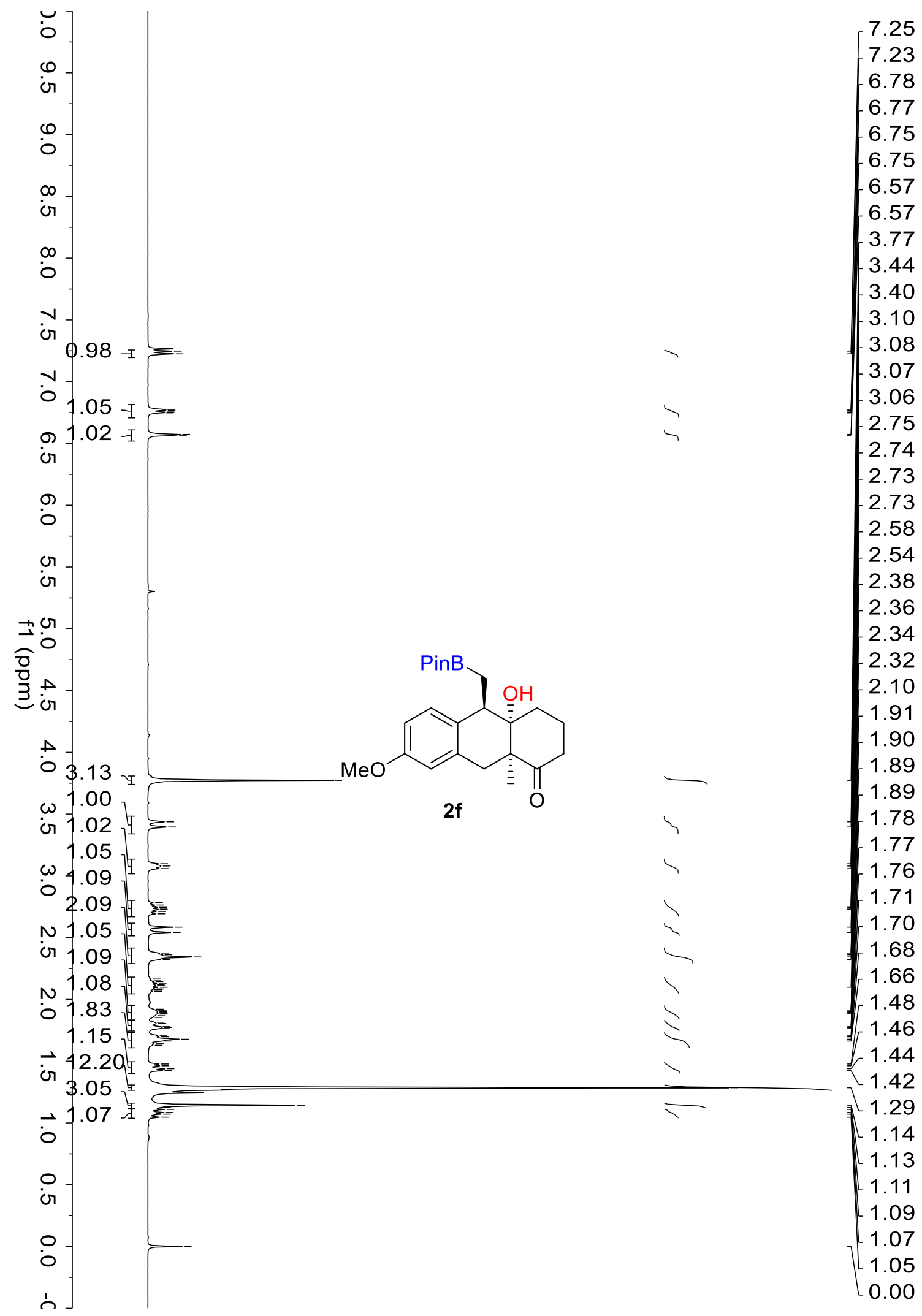




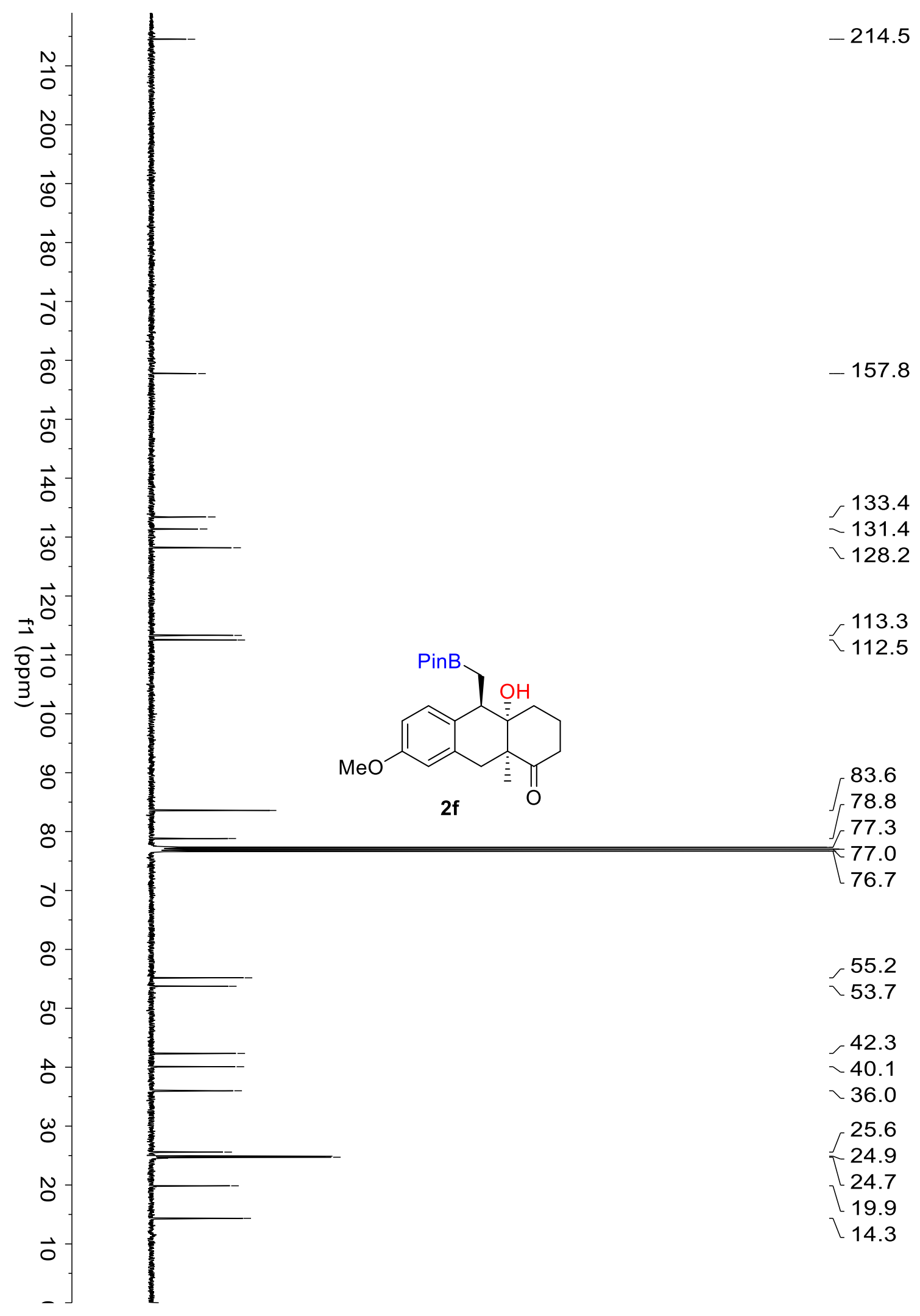




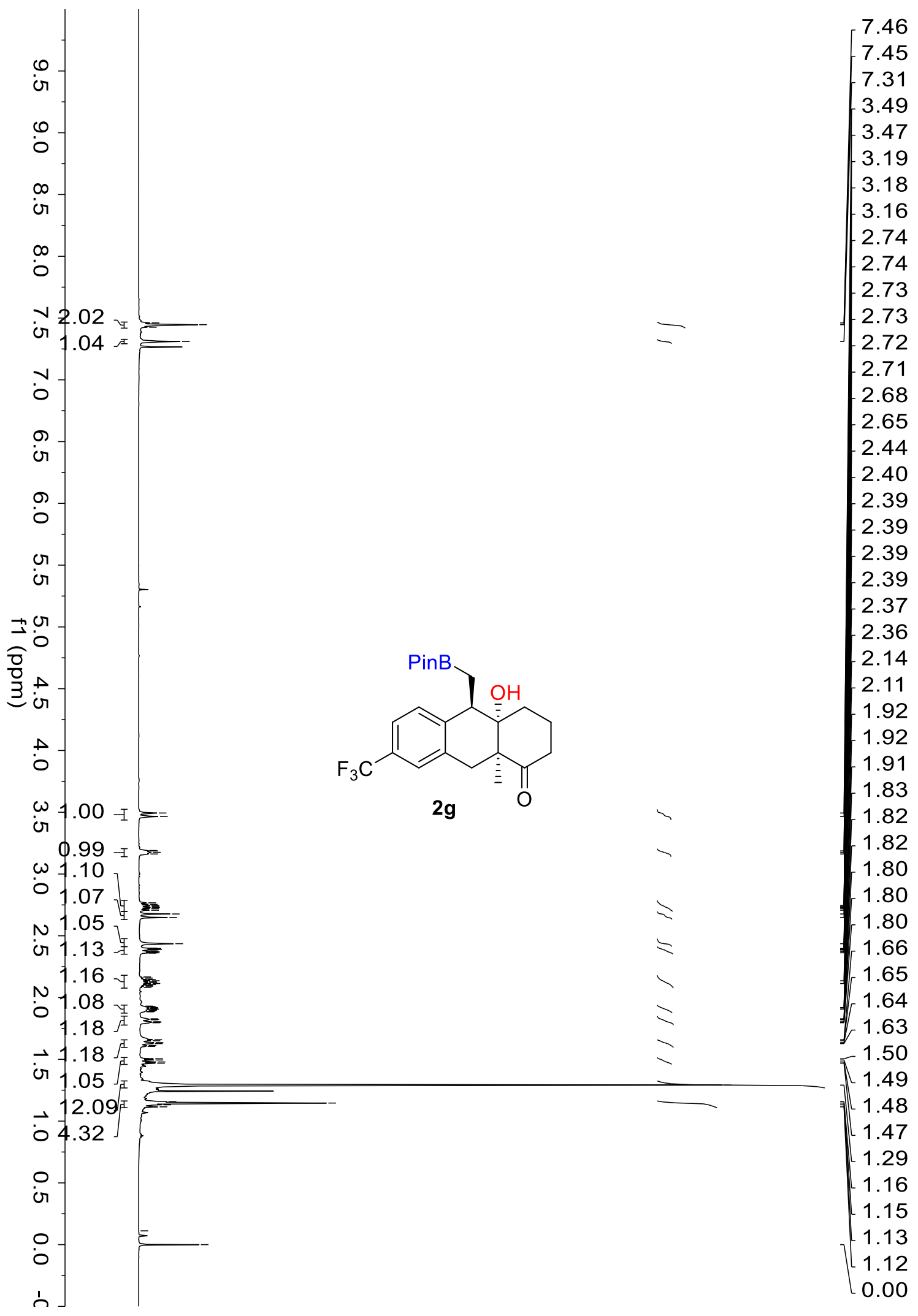




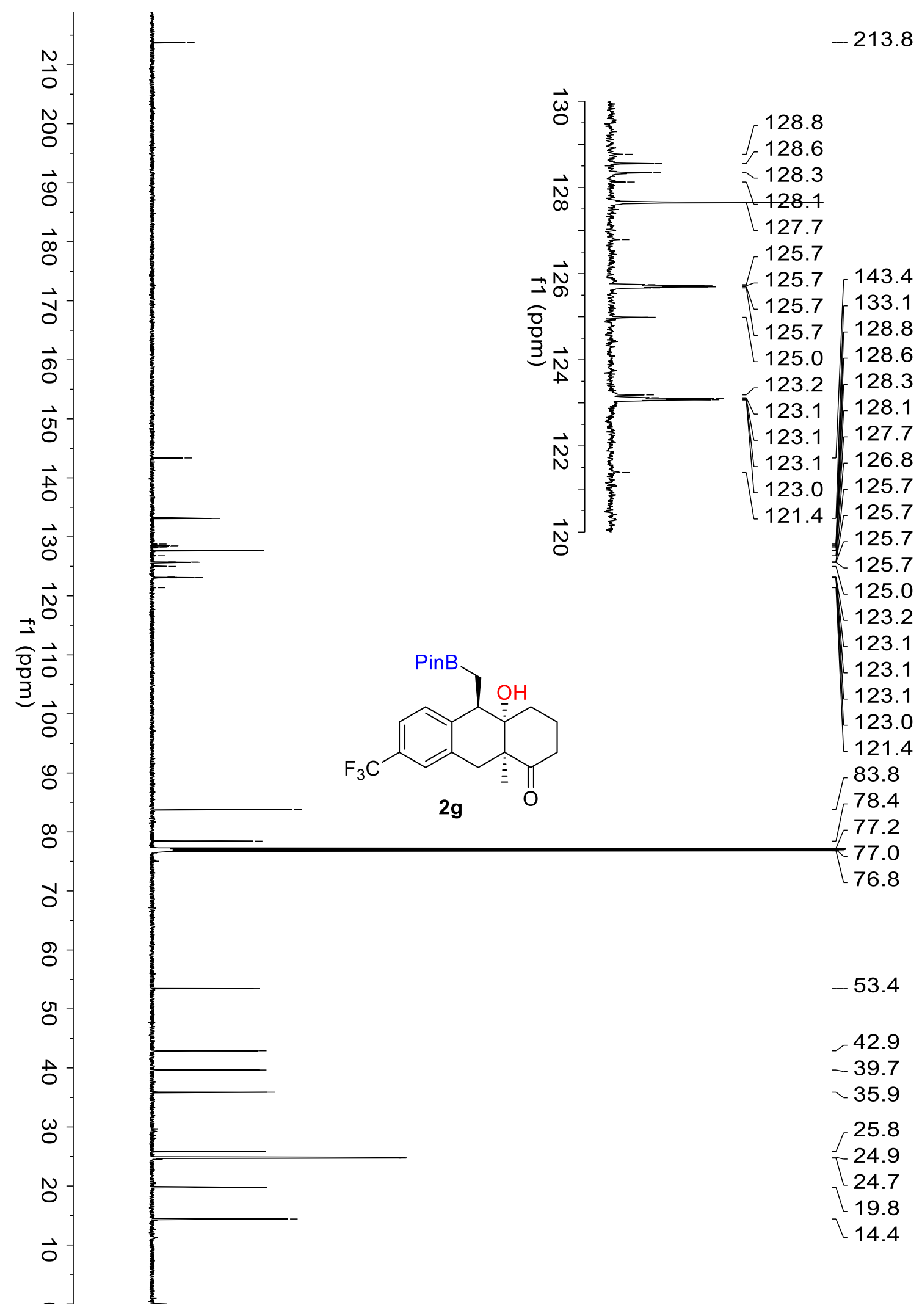




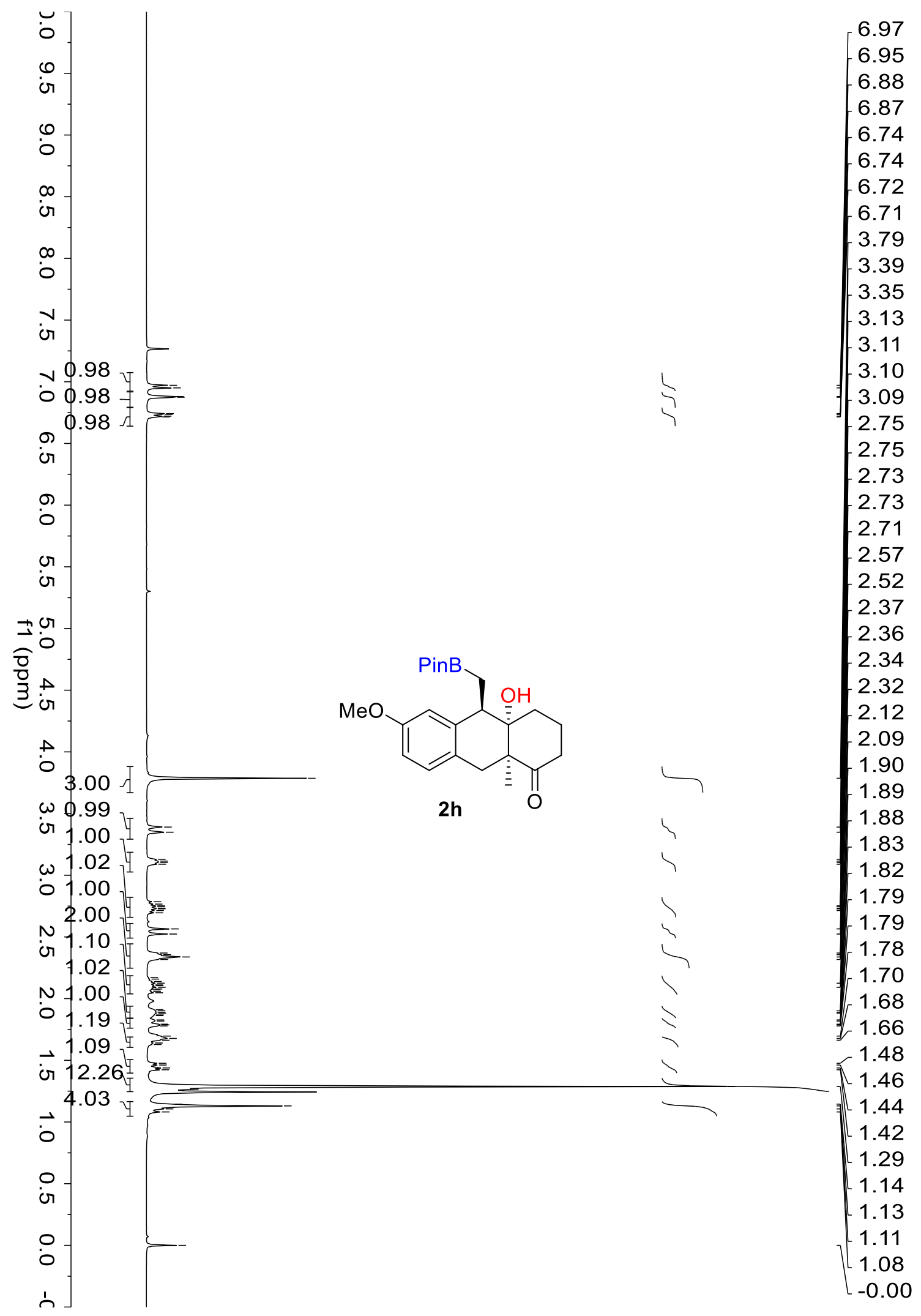




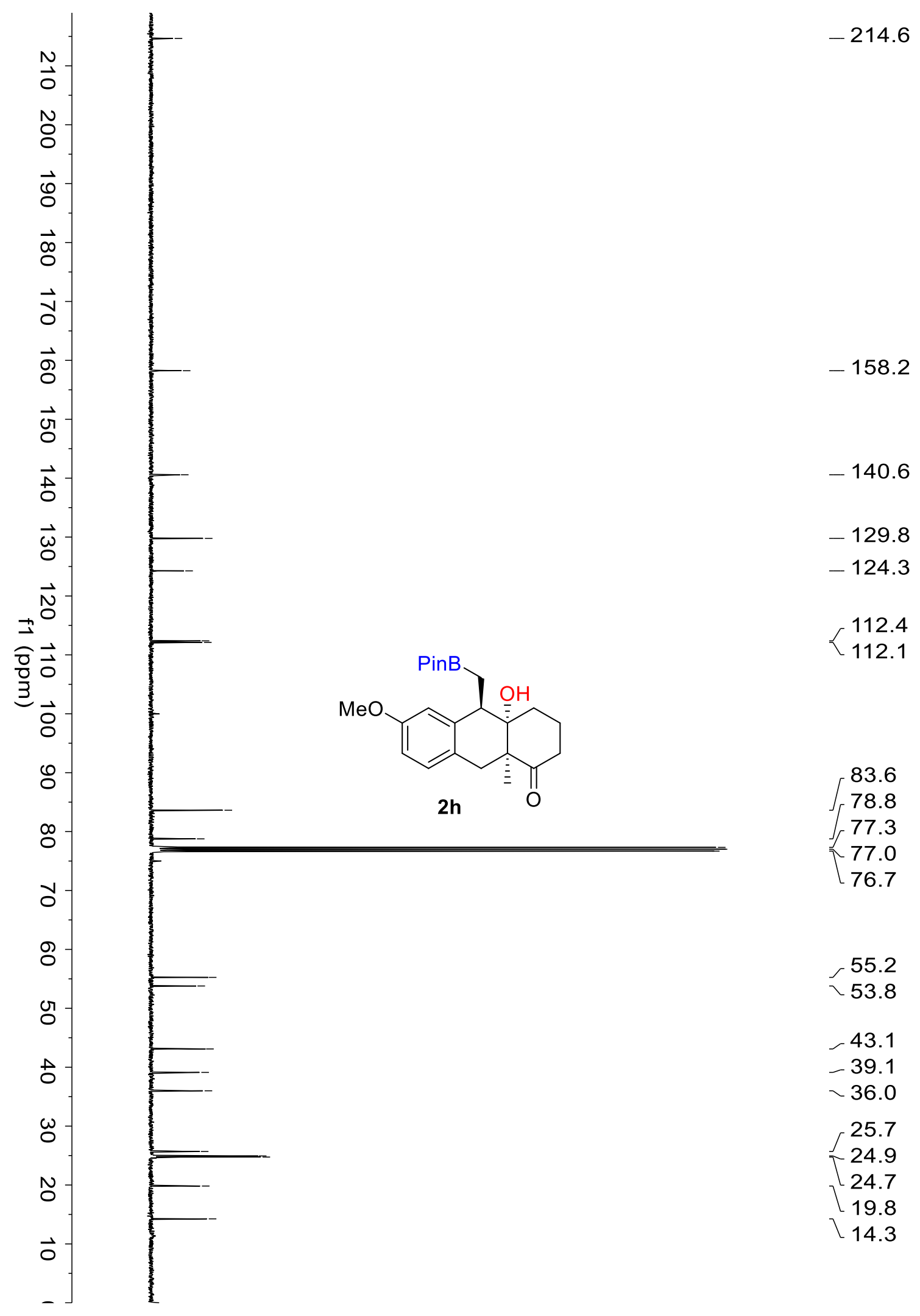



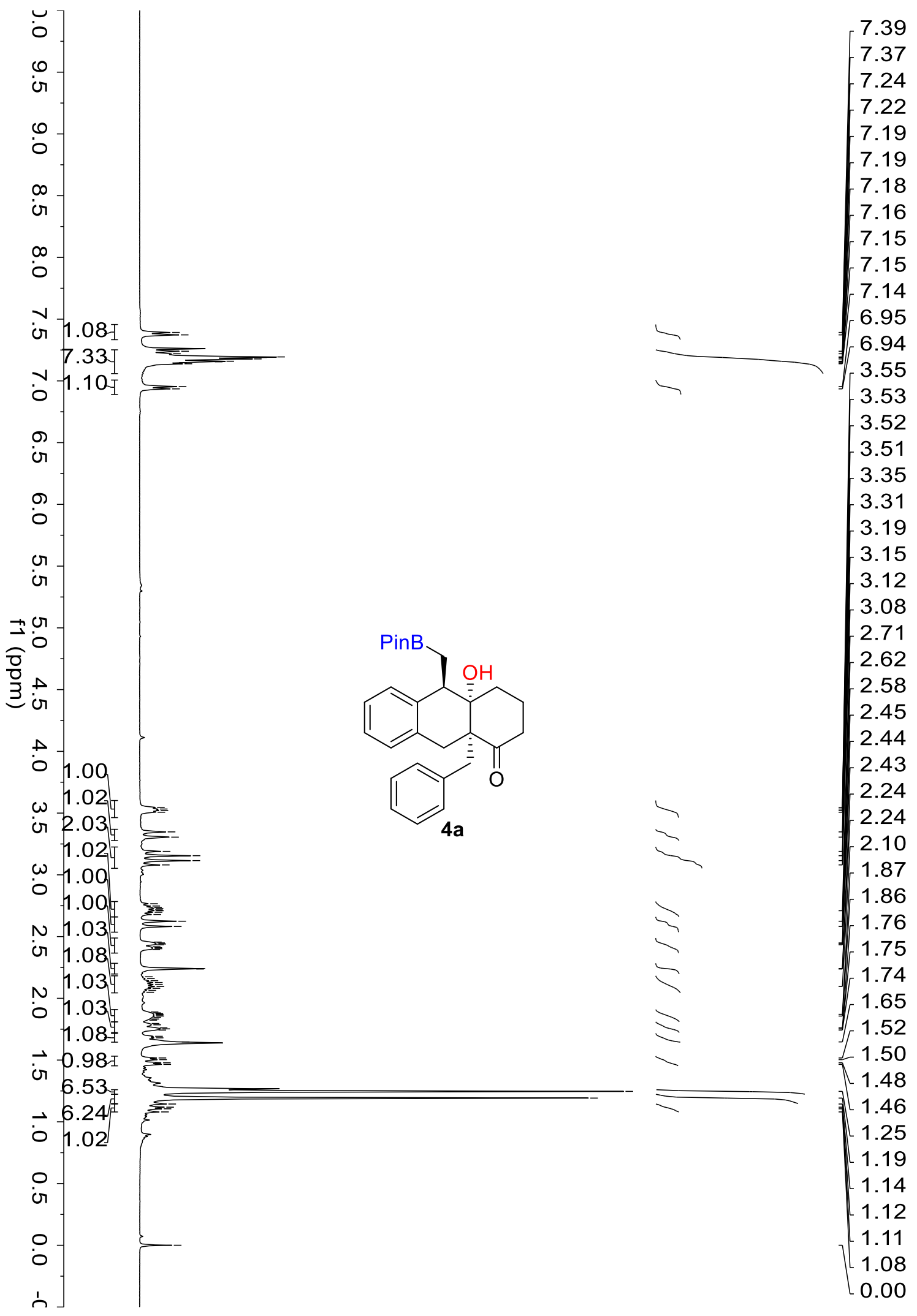


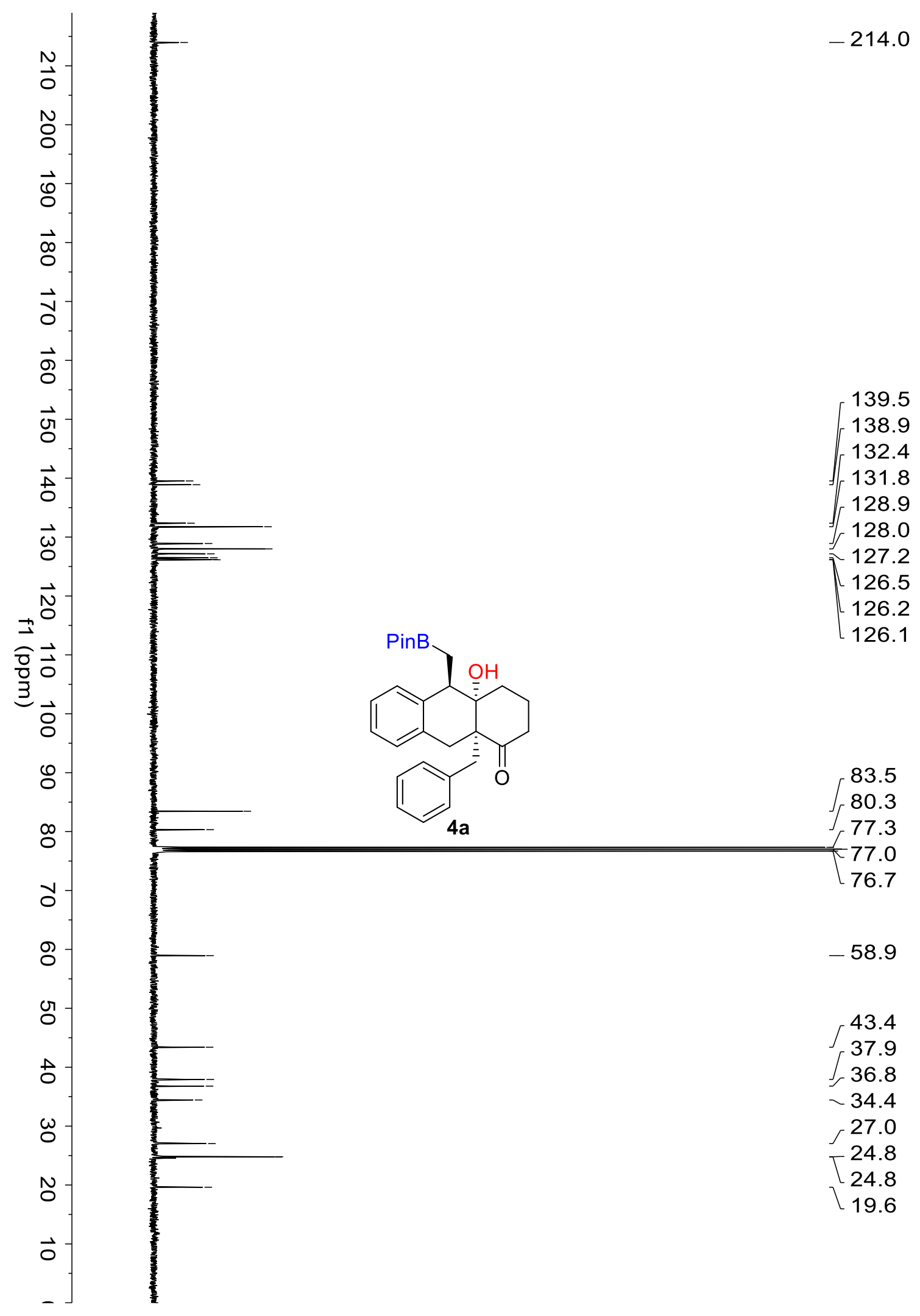




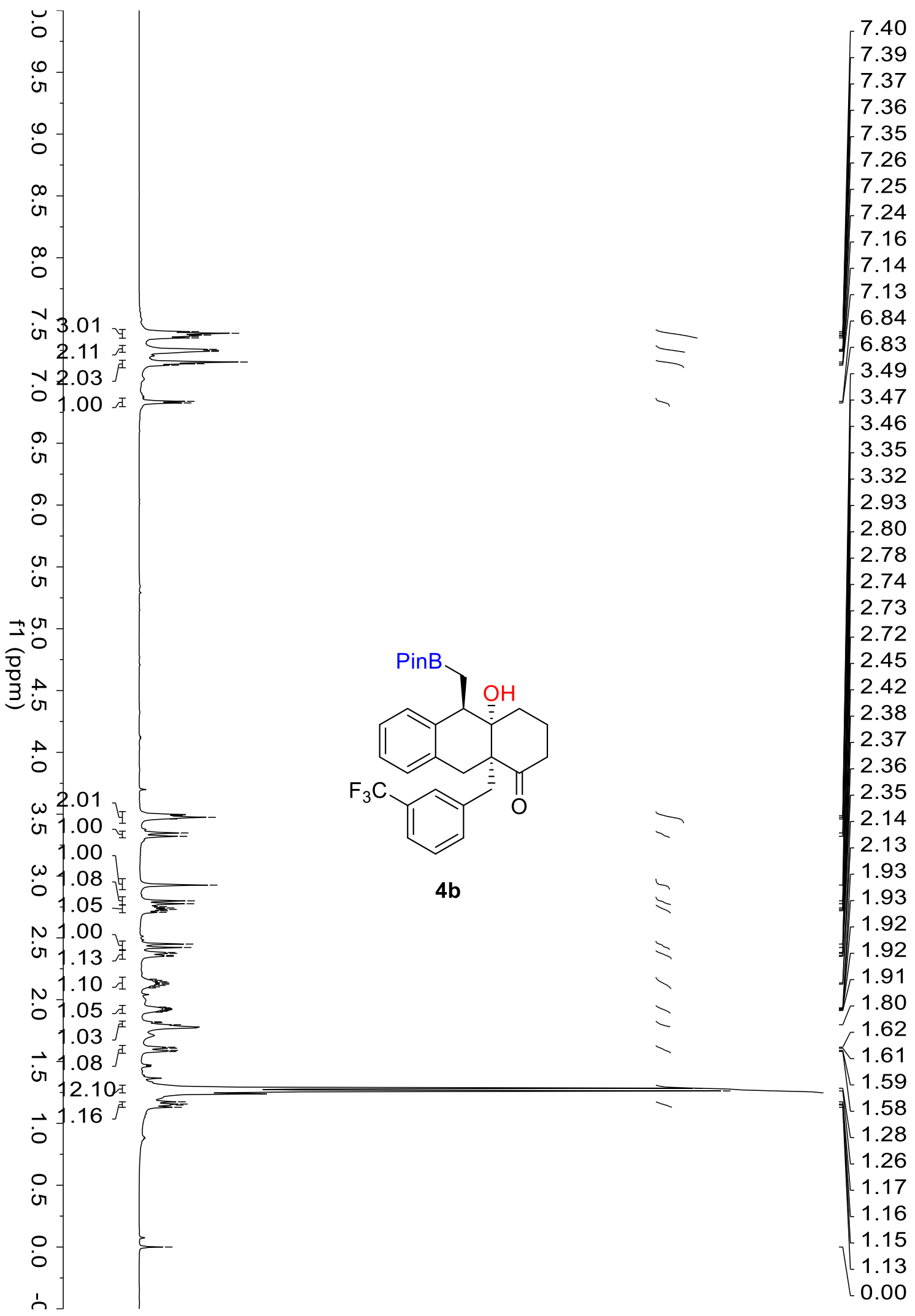




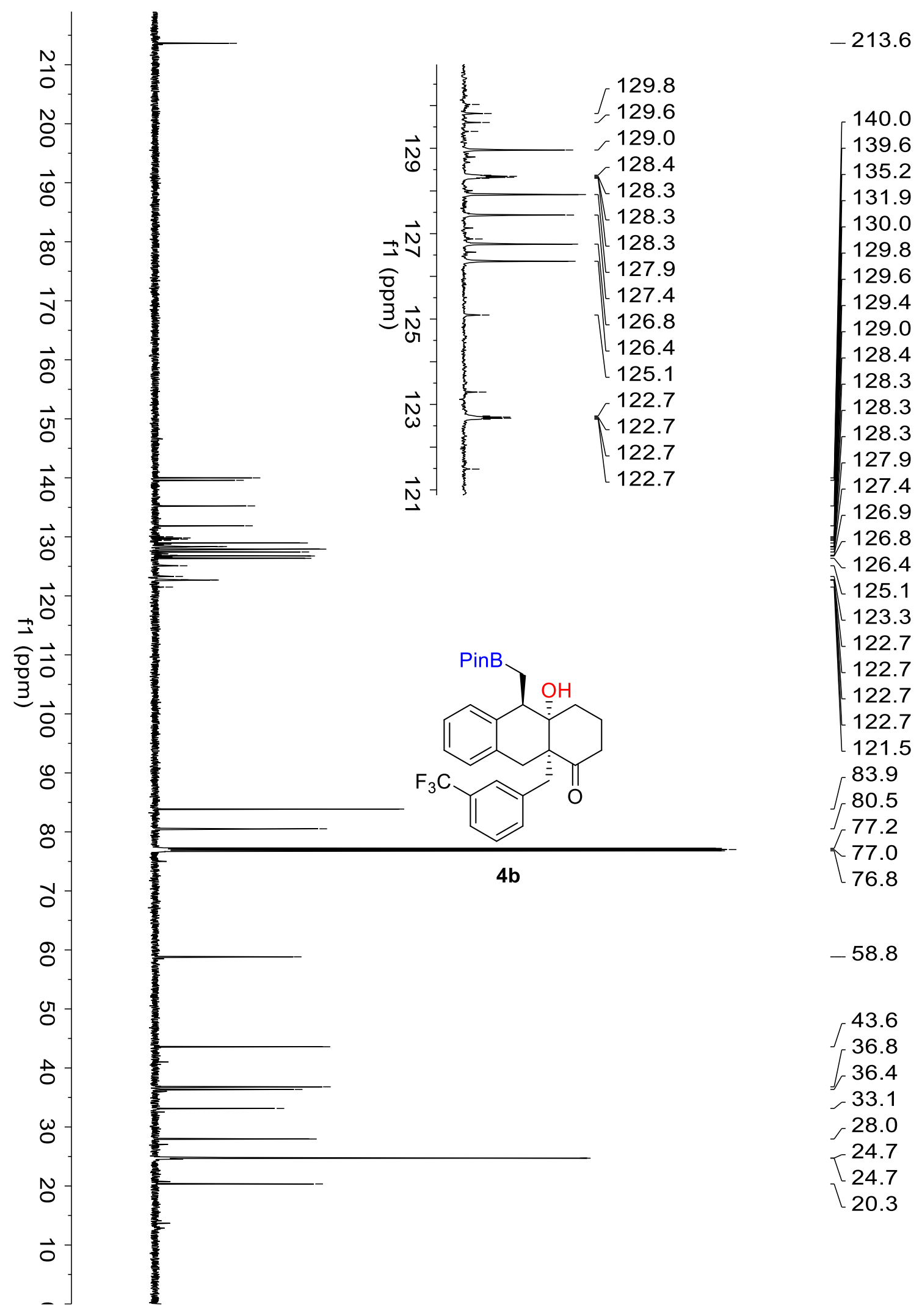




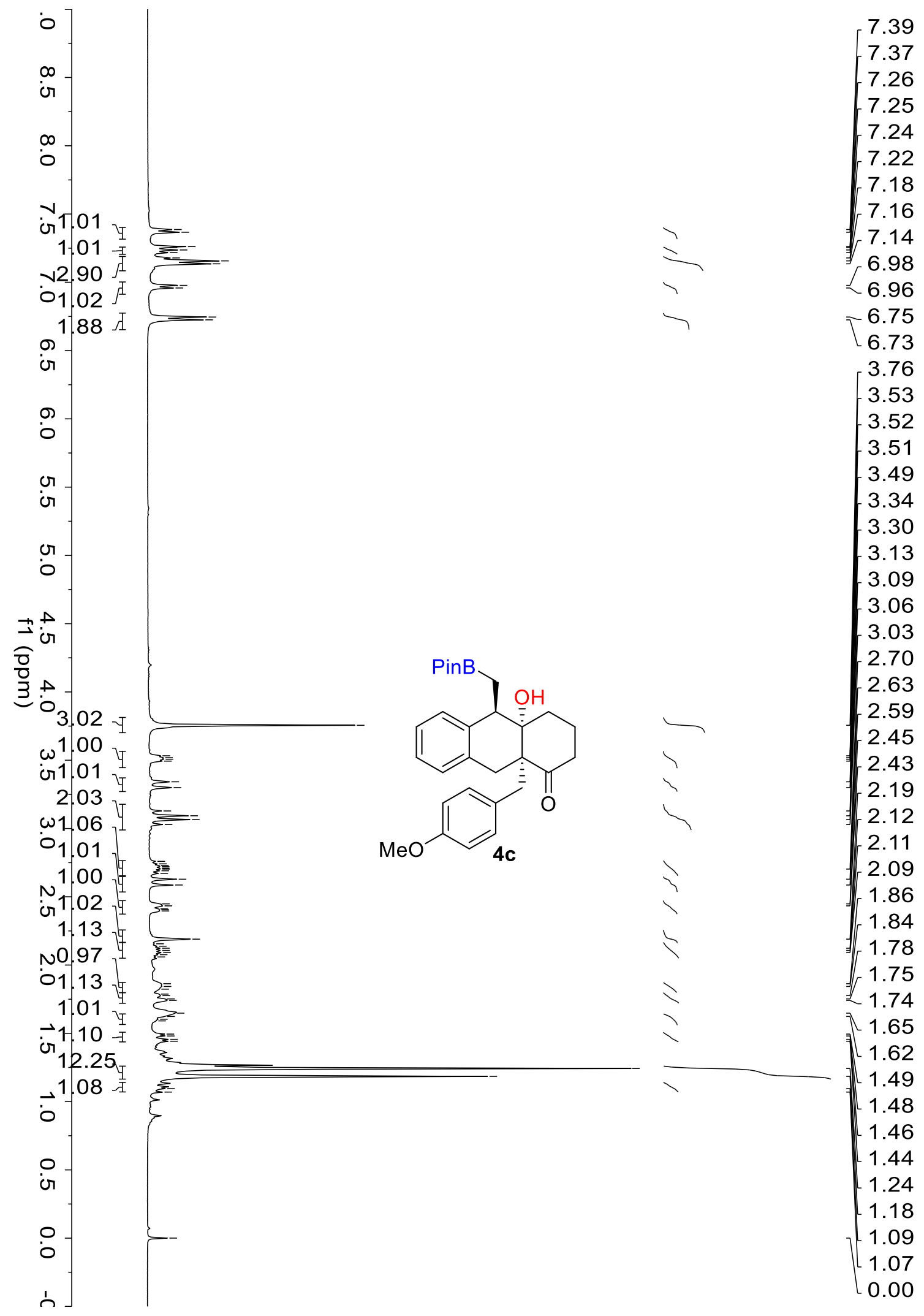




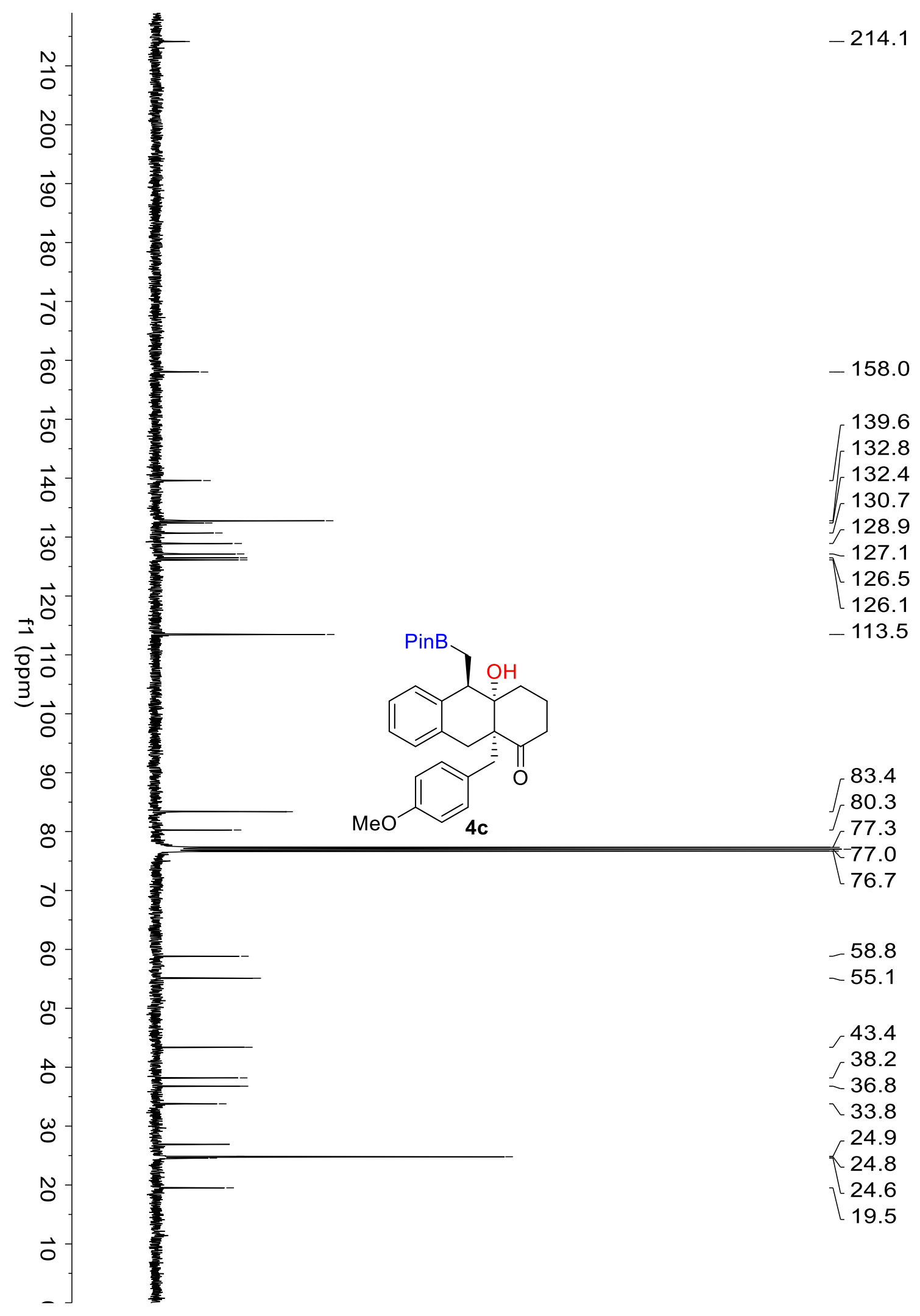




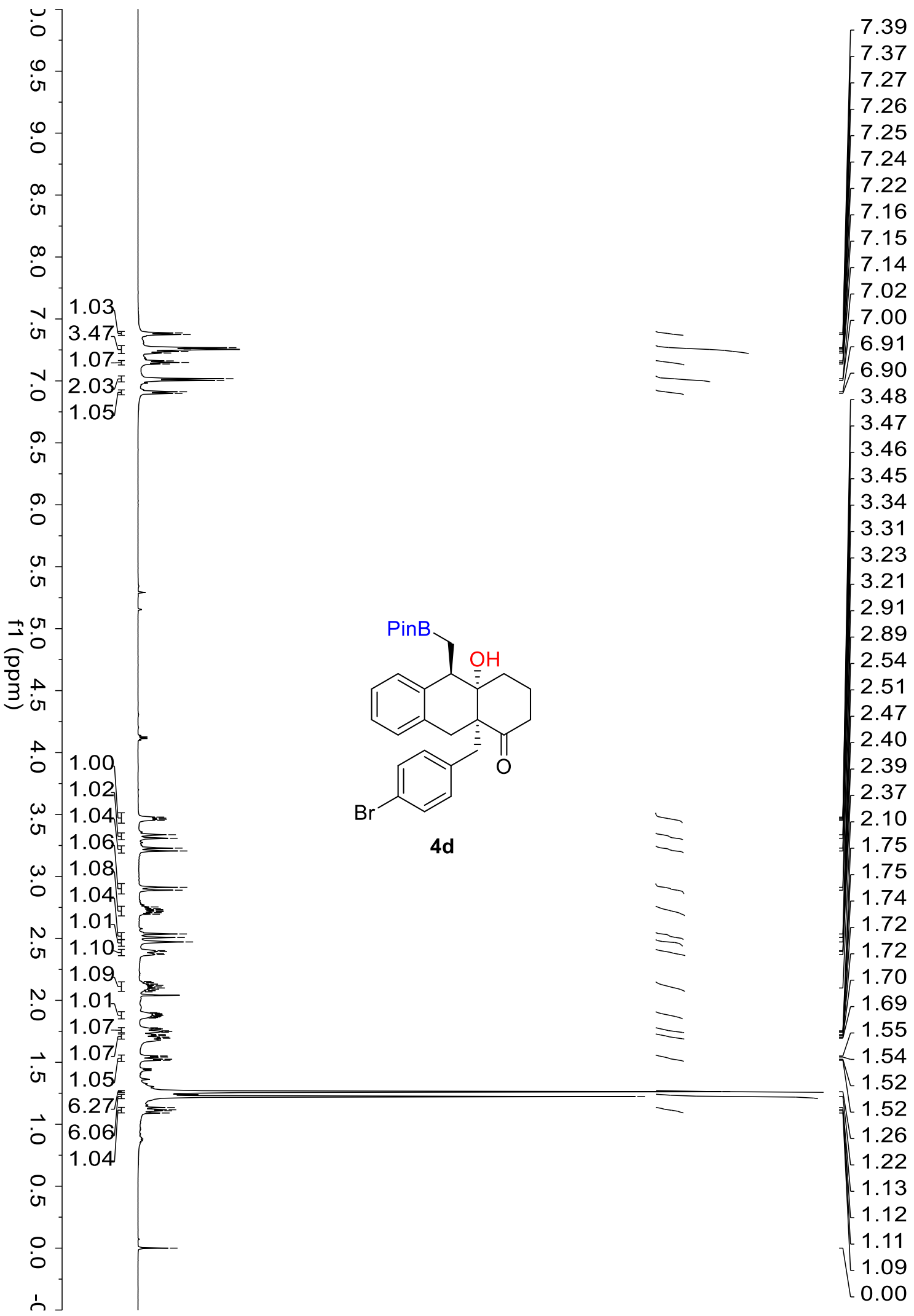




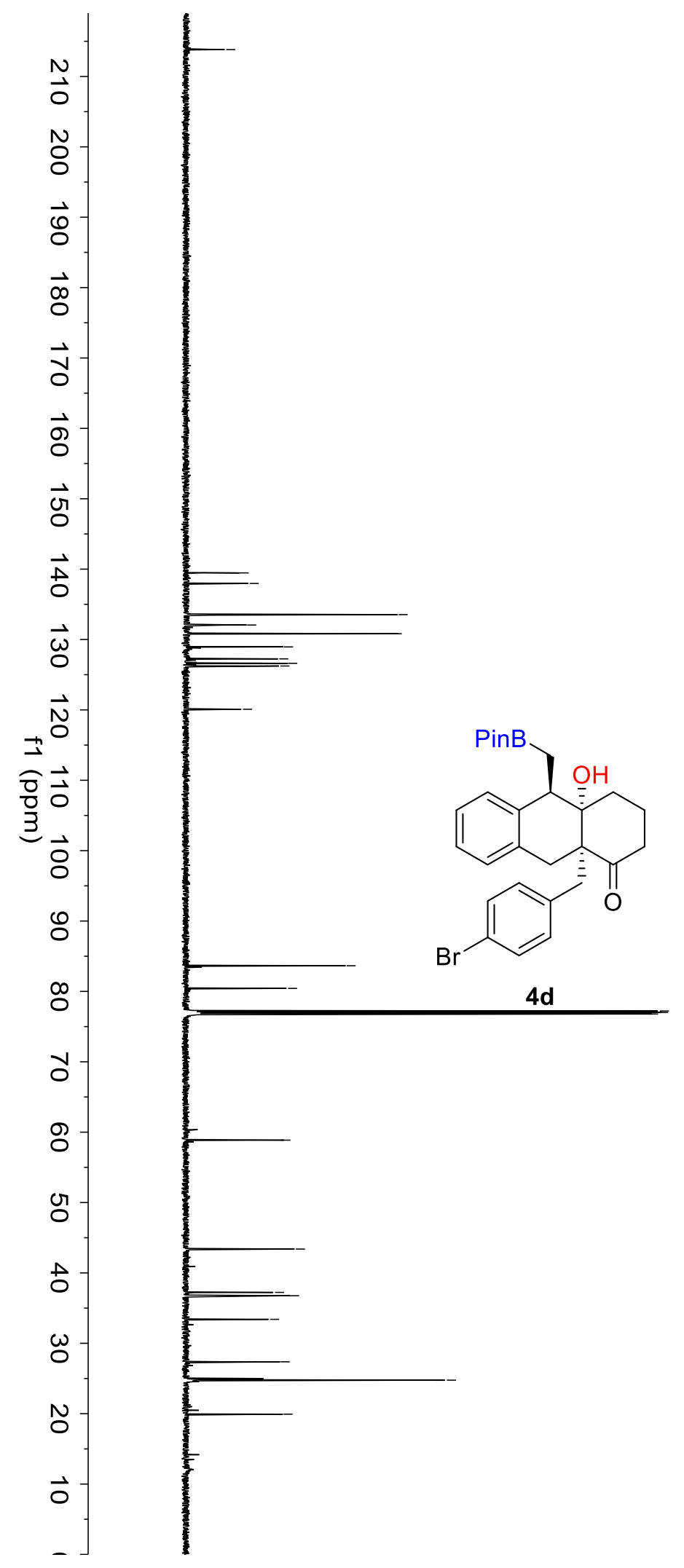

139.5

138.0

133.5

132.1

130.8

128.9

127.3

126.6

126.2

120.1

83.6

80.4

77.2

77.0

76.8

$-58.9$

43.4
37.2

36.8

$-33.4$

27.4

24.8

24.7

\19.9 

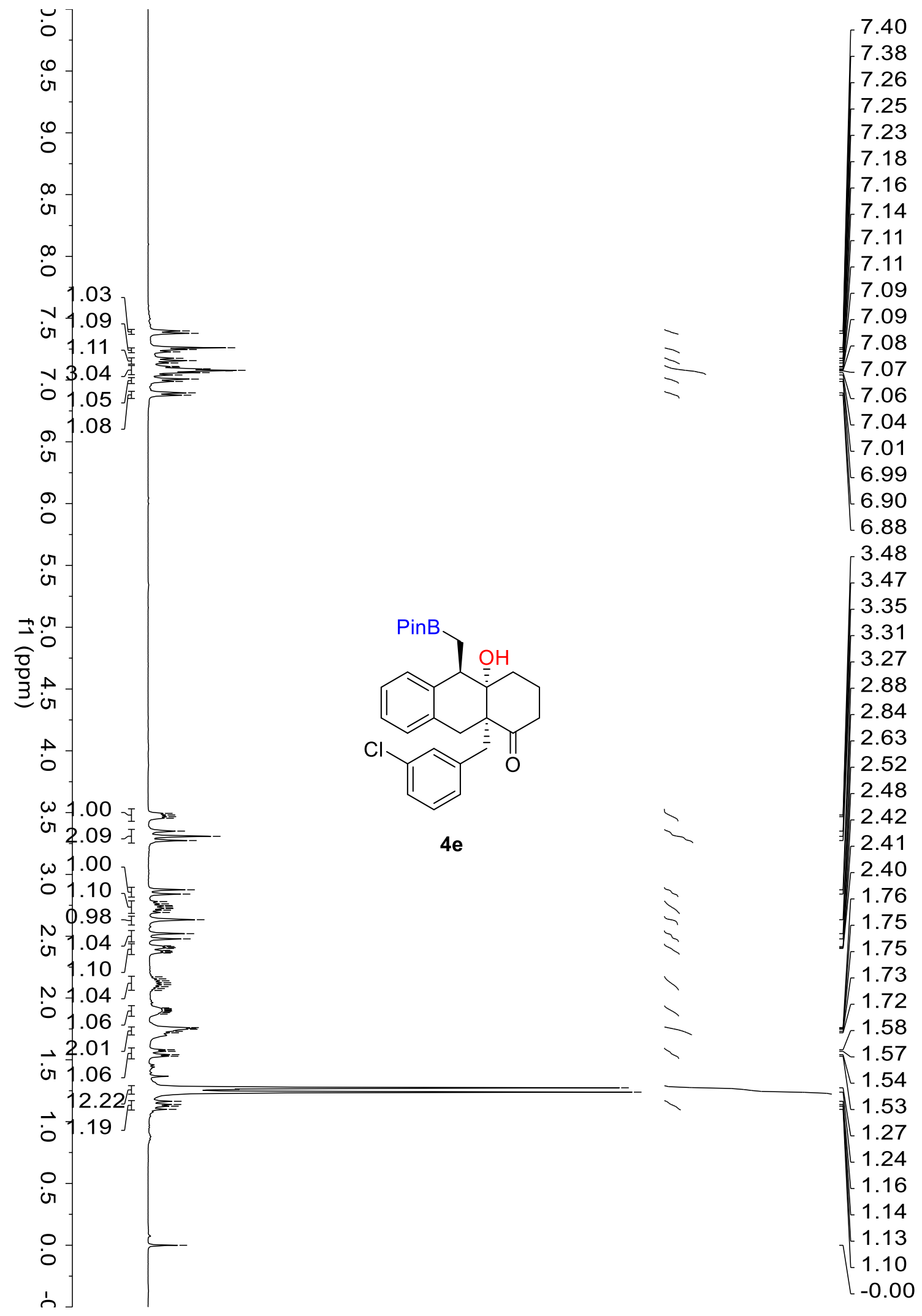


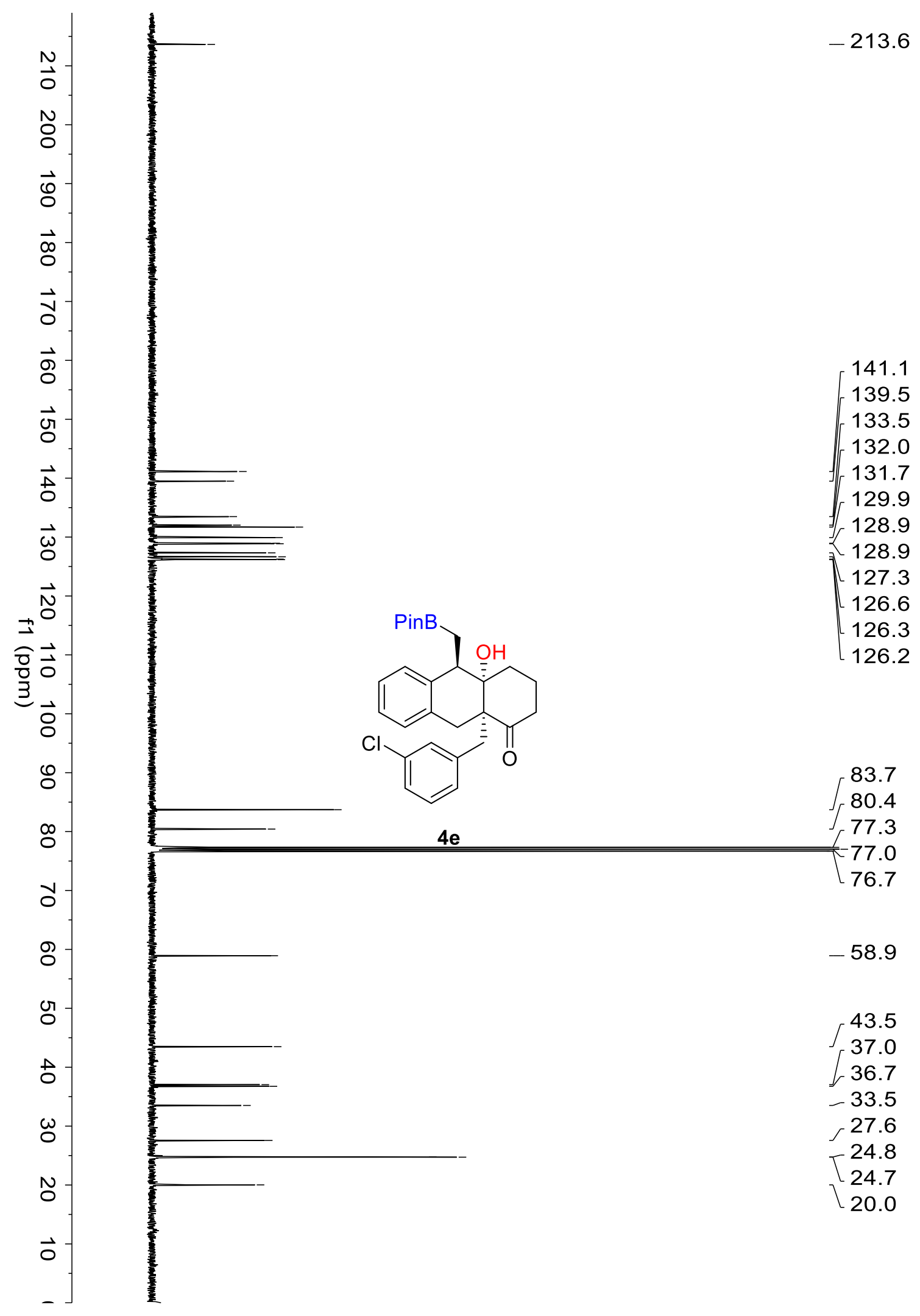




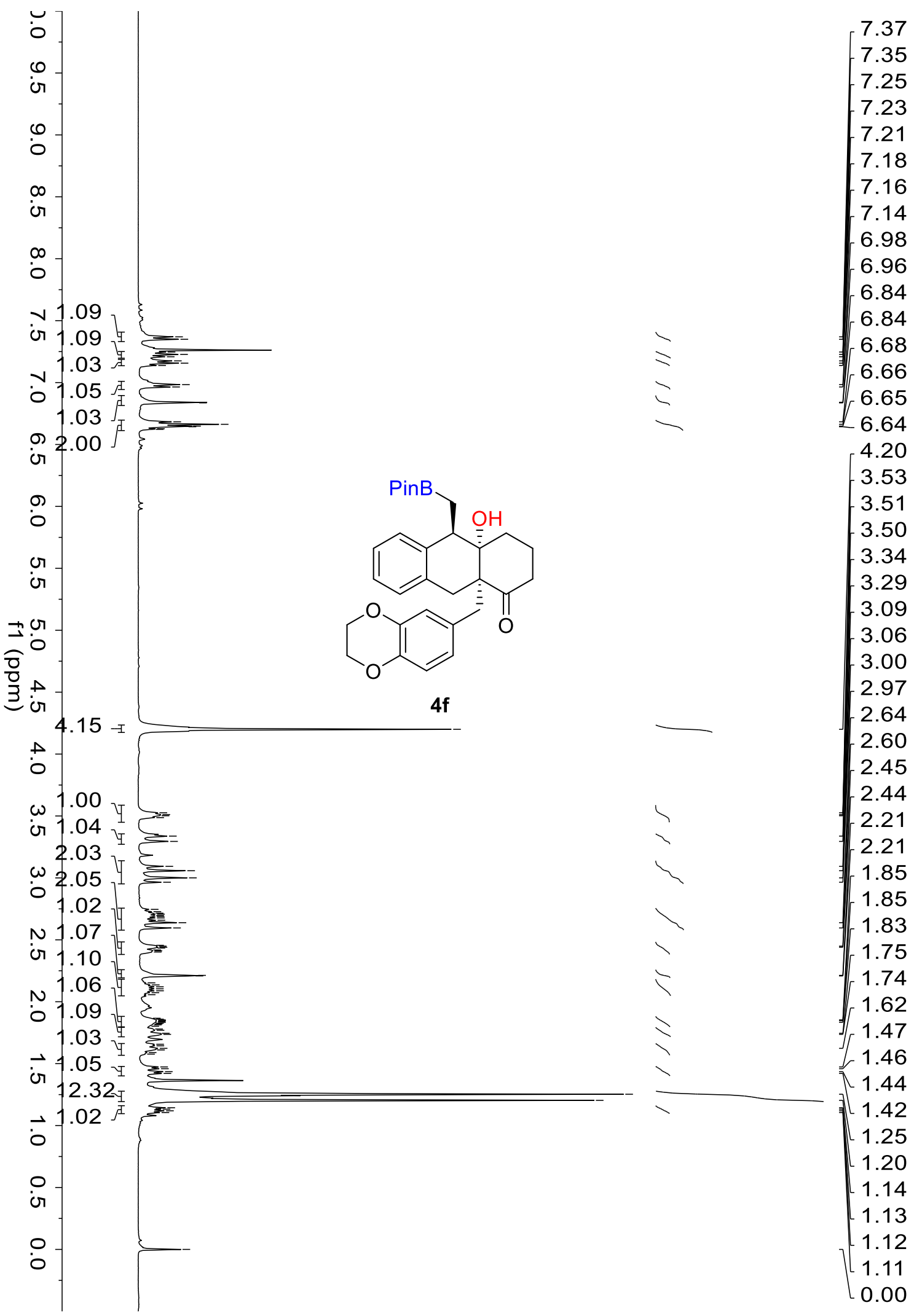




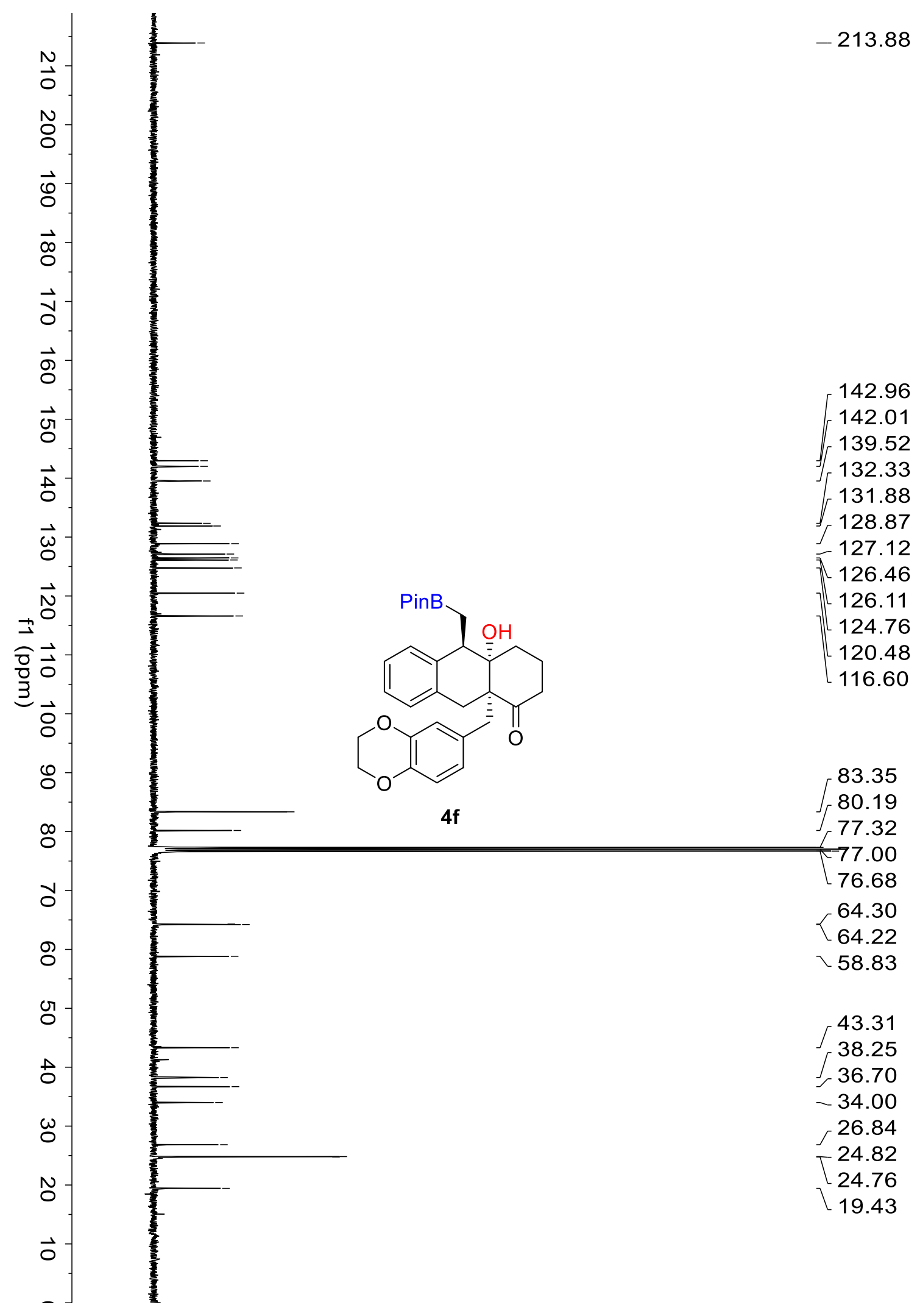




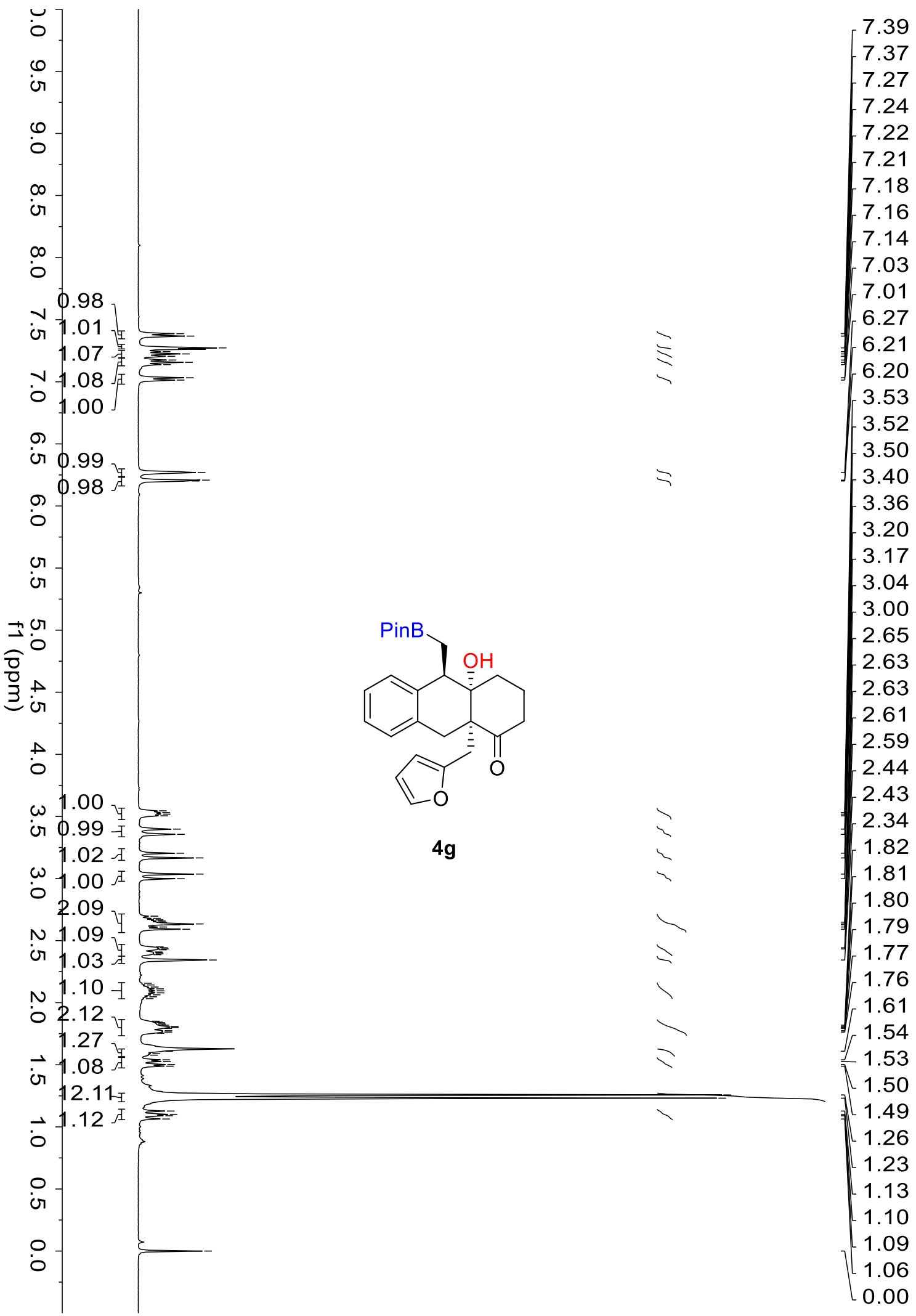




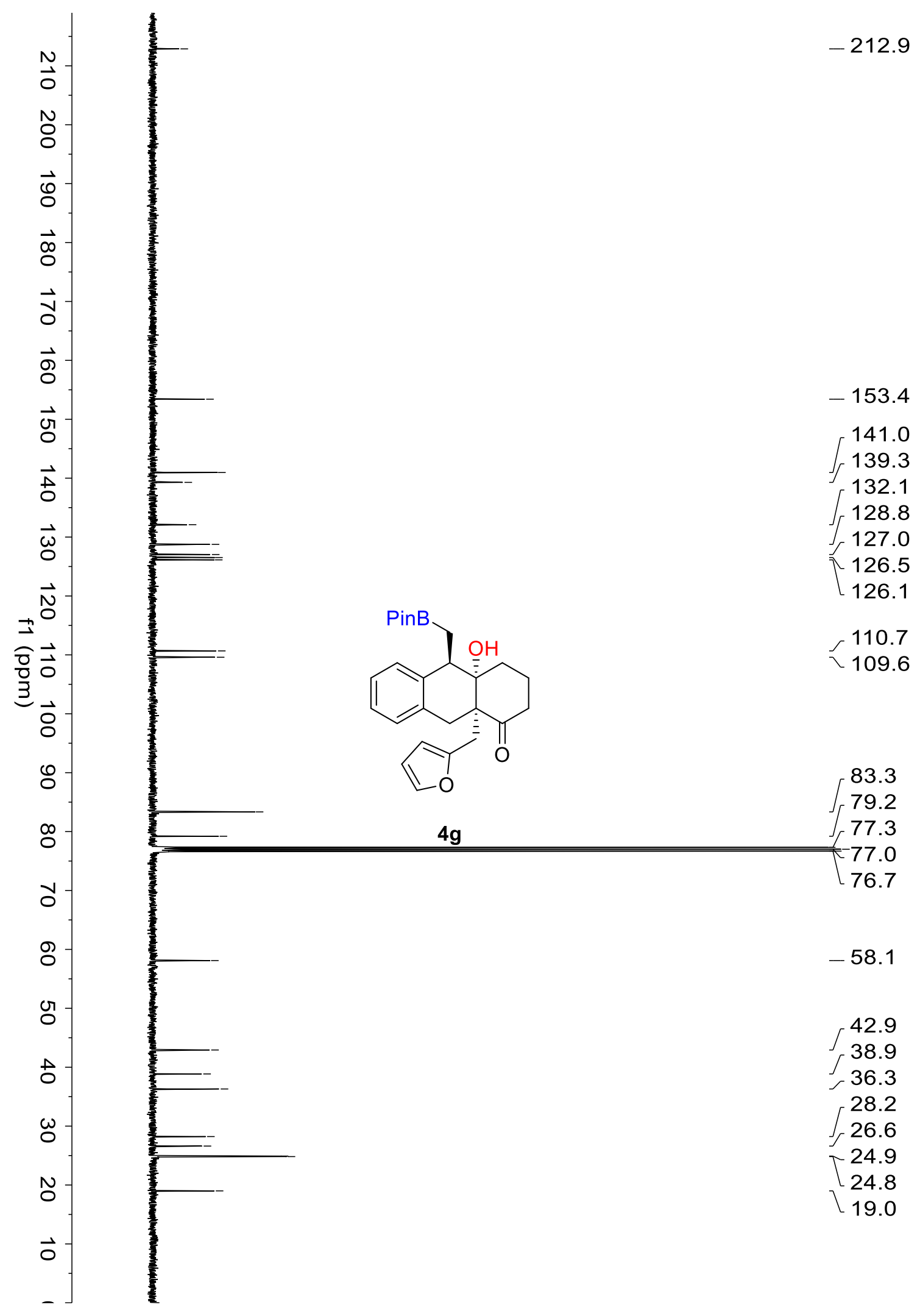




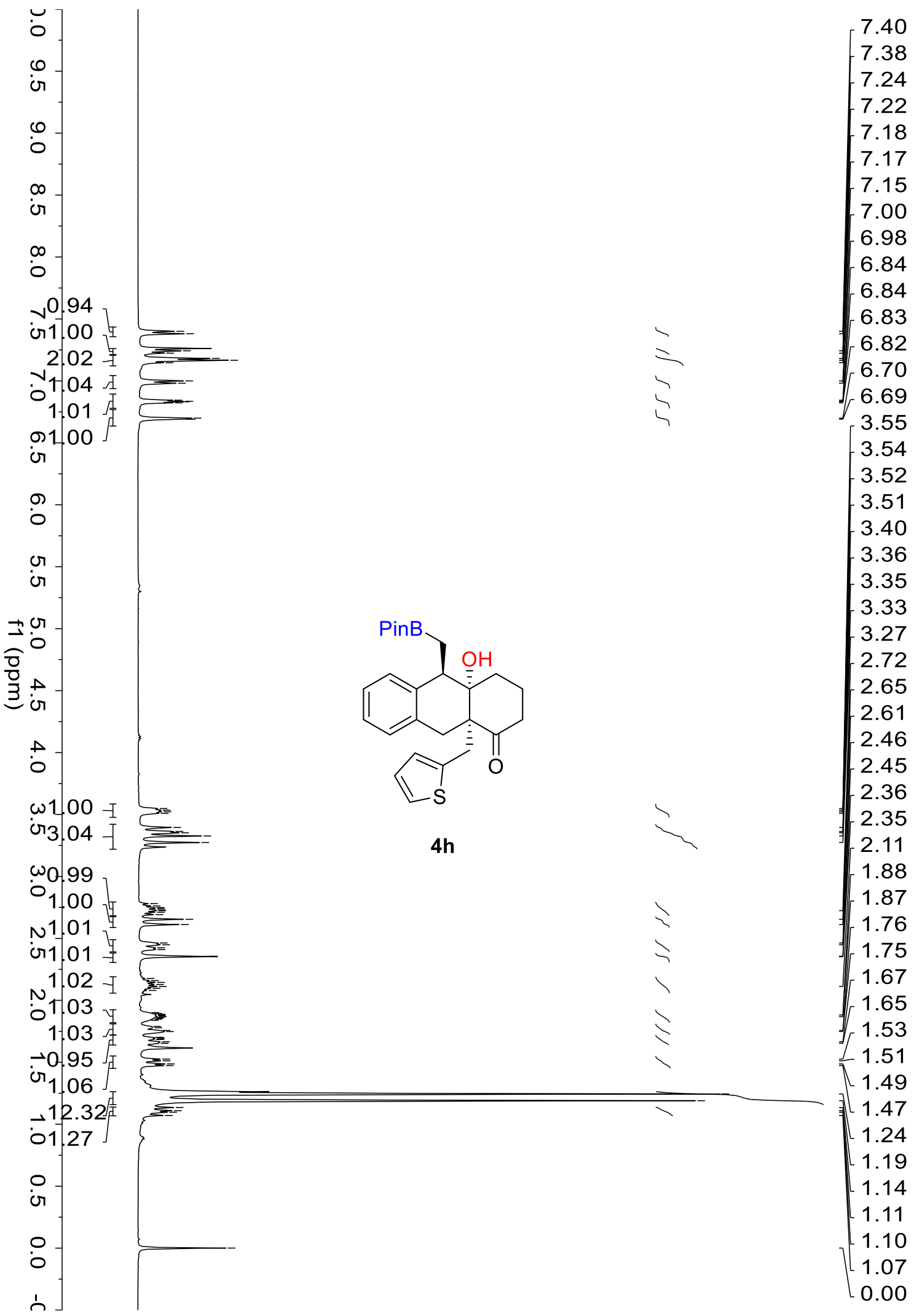




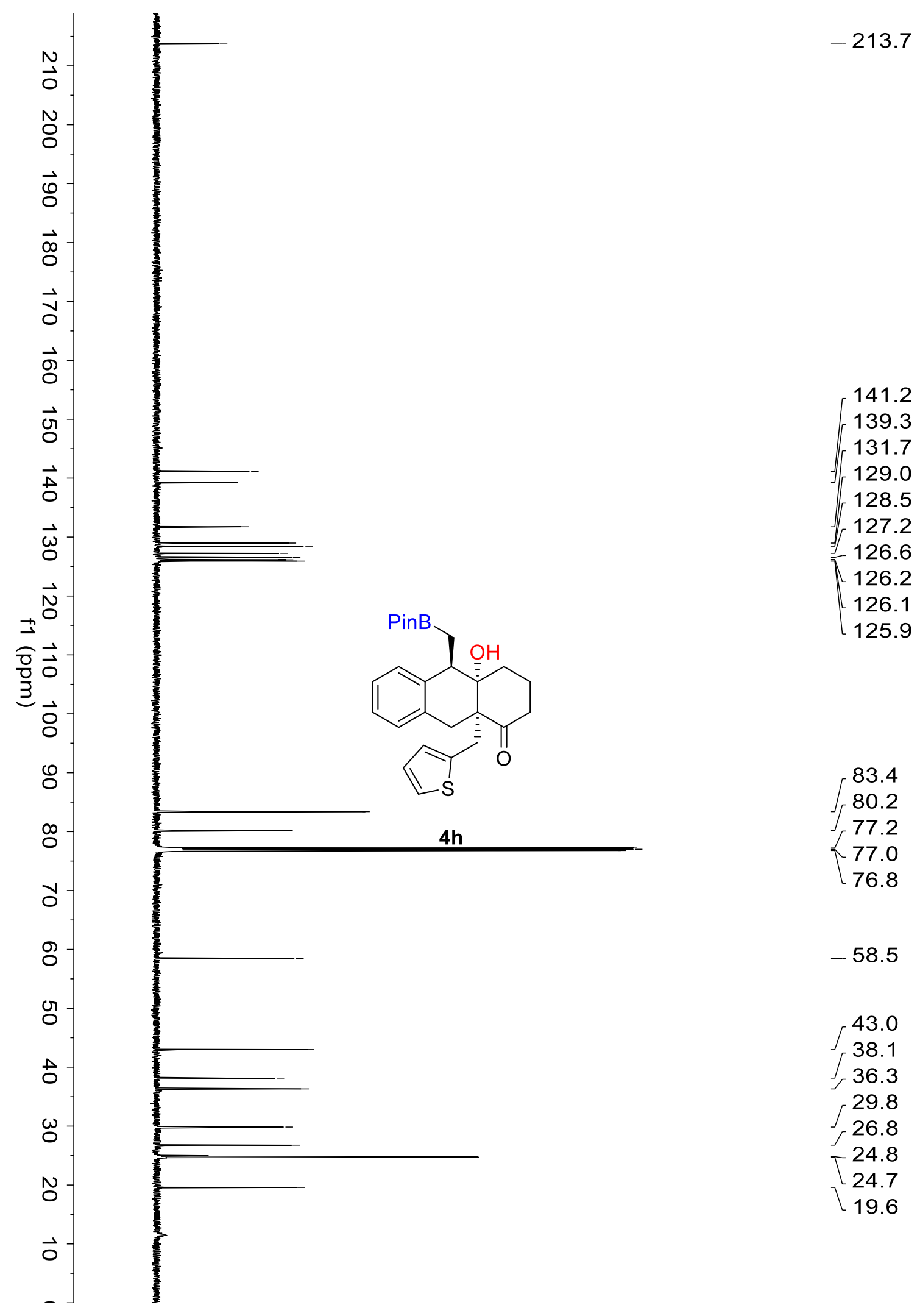




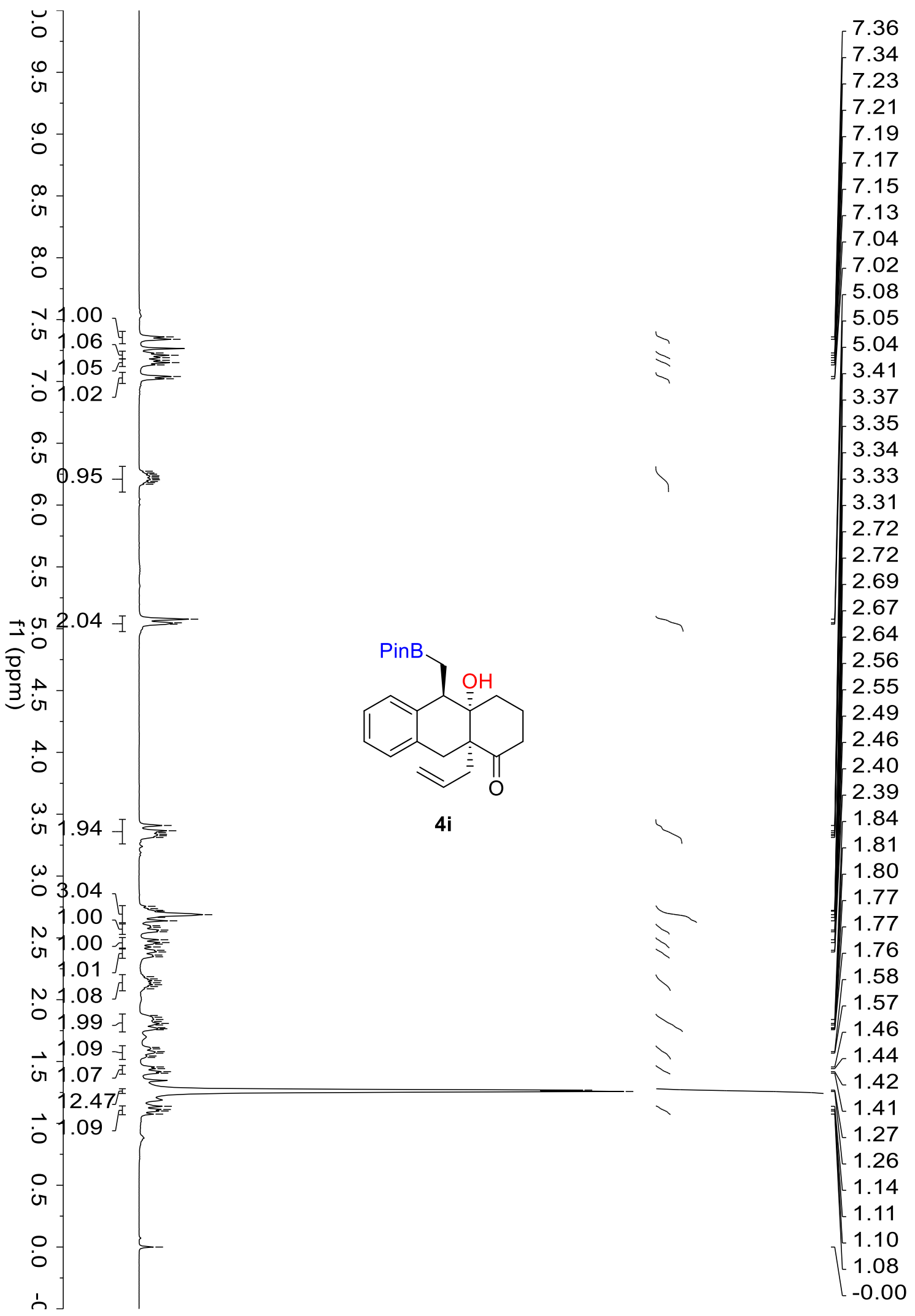




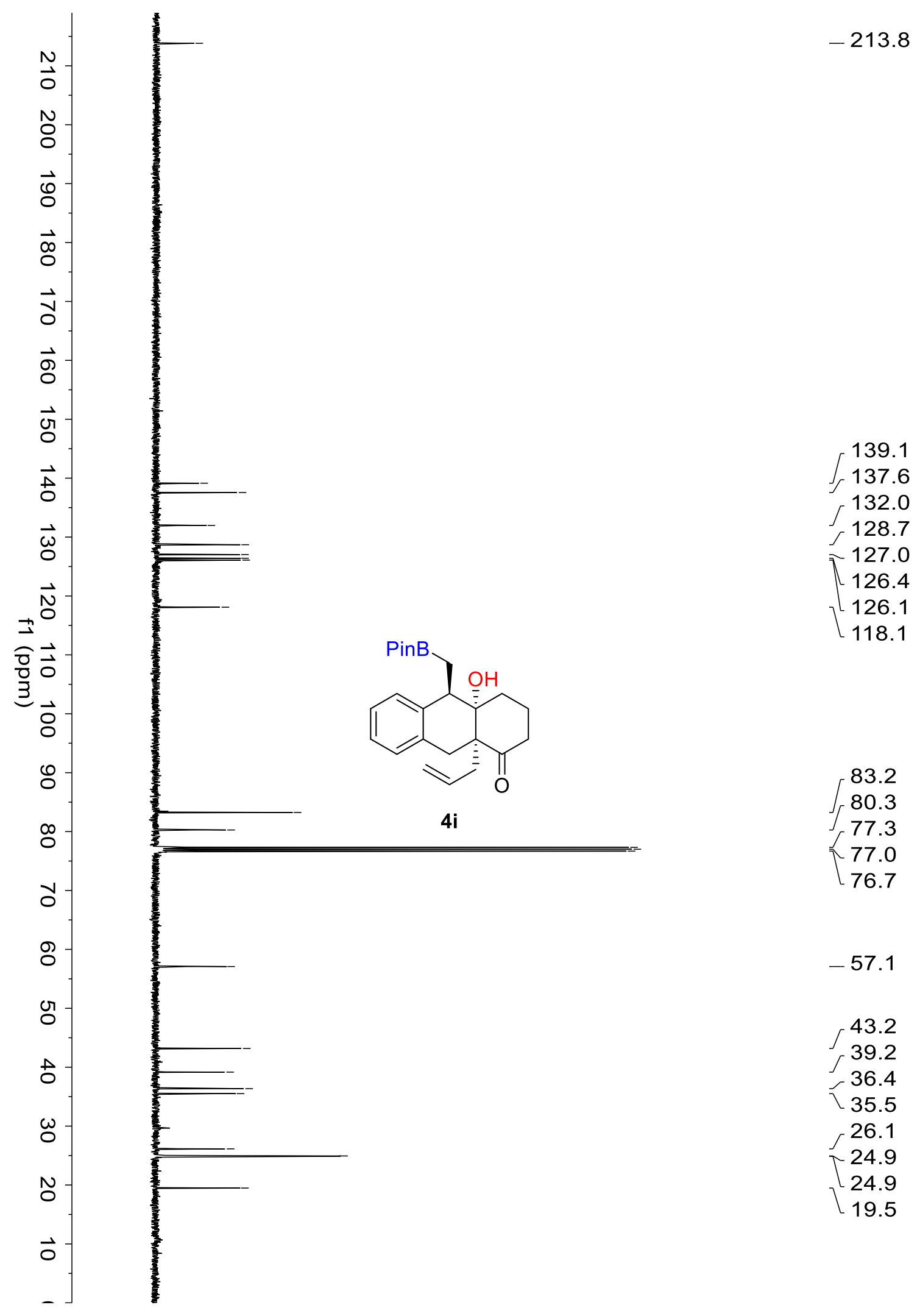




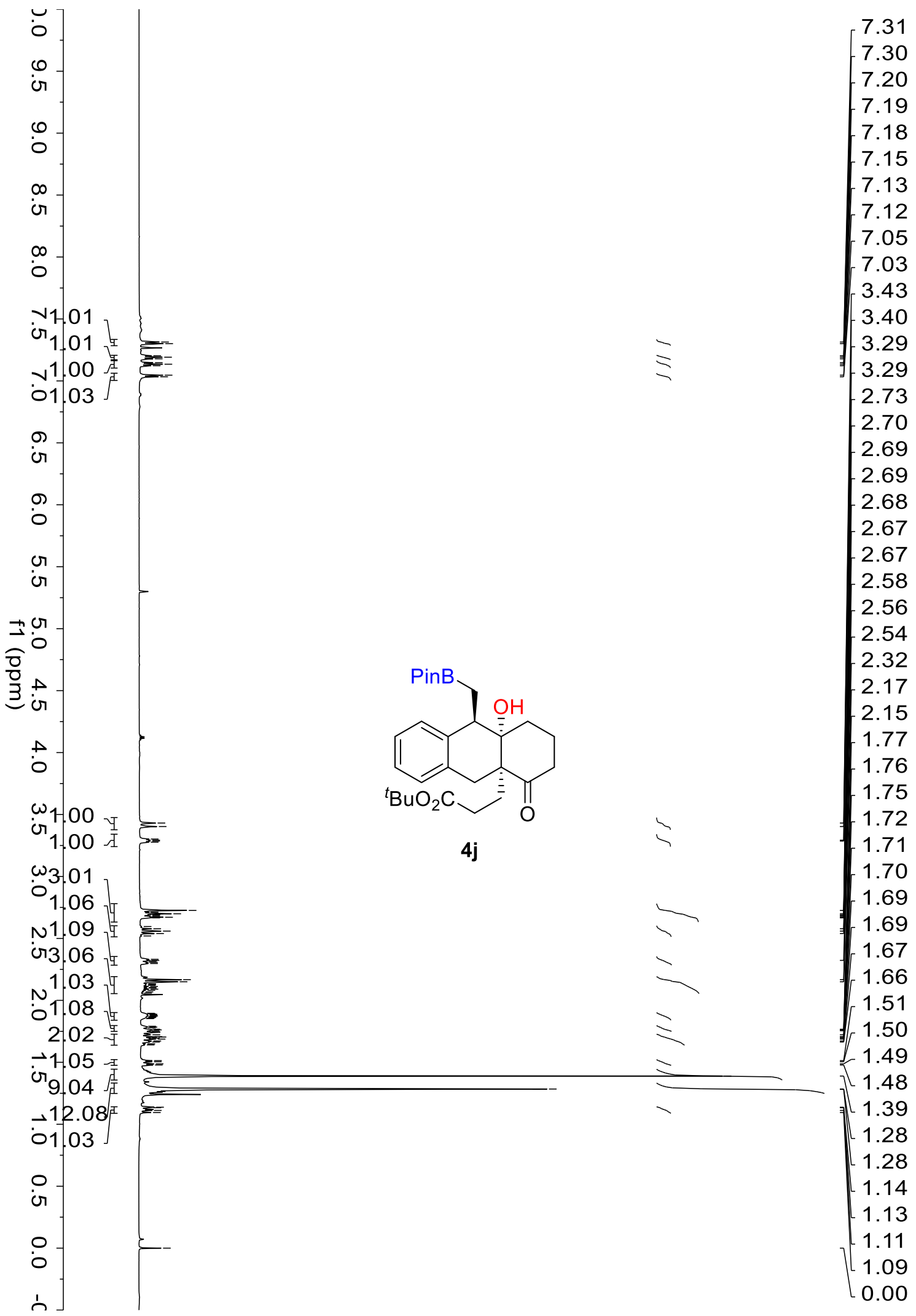




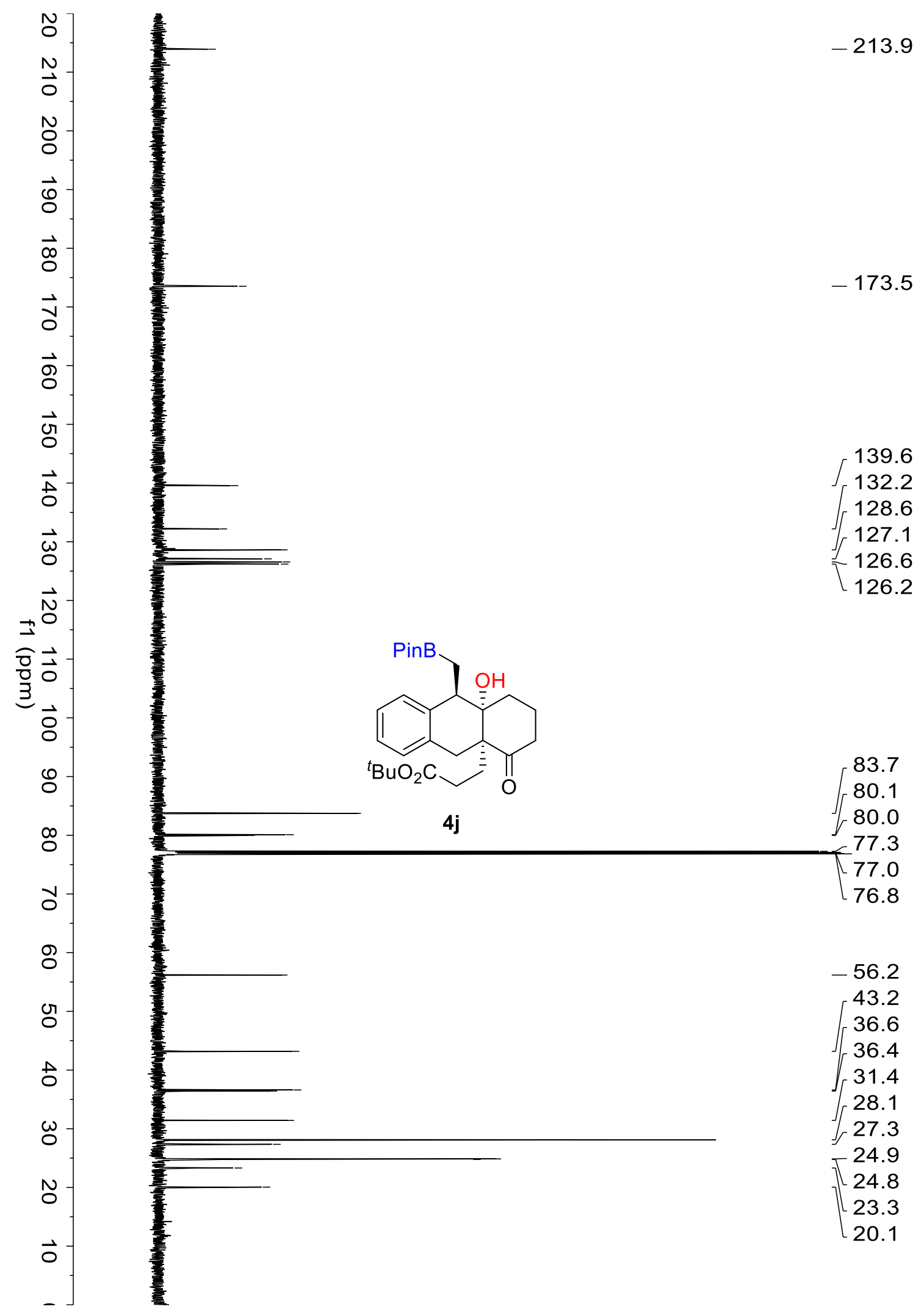




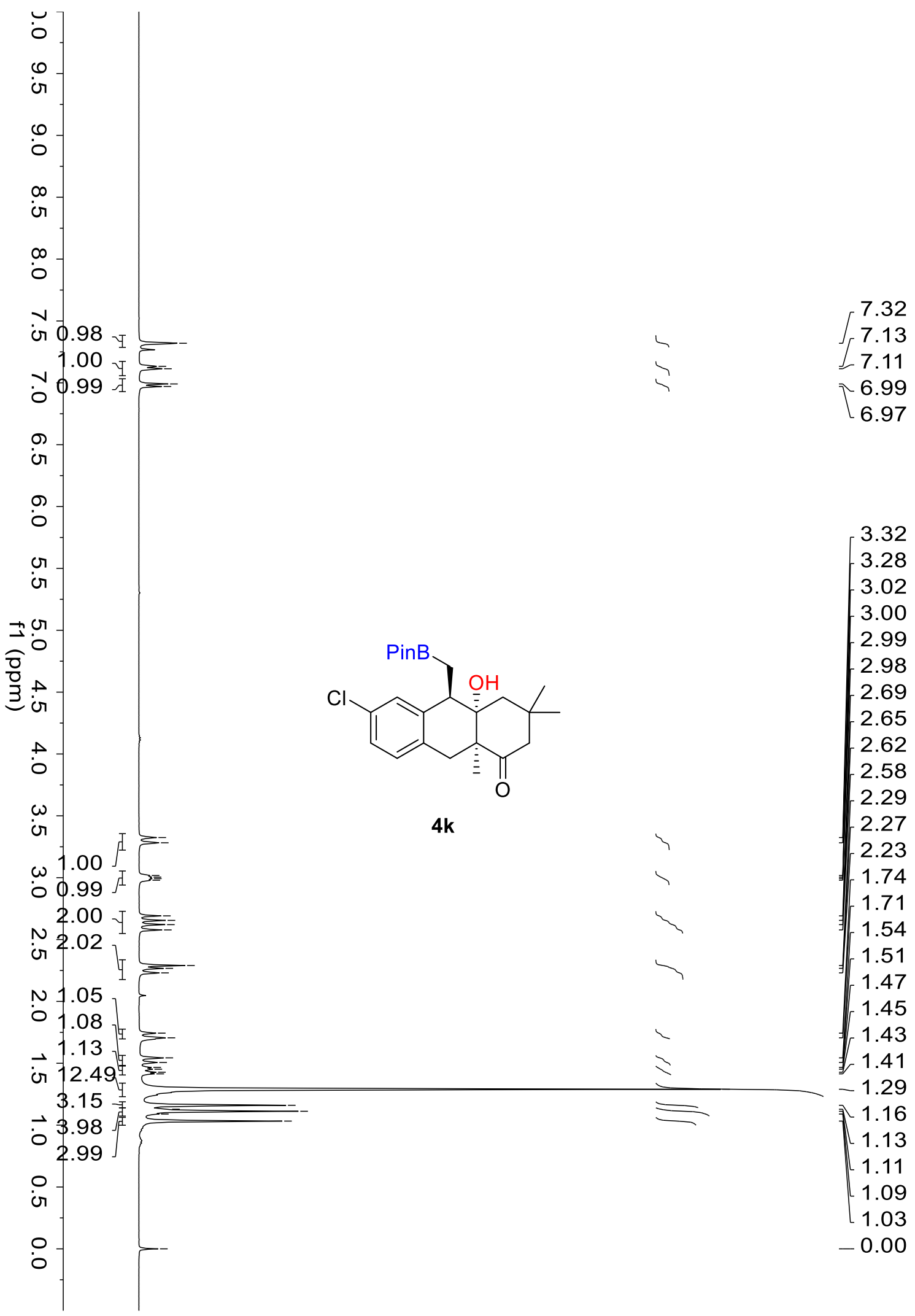




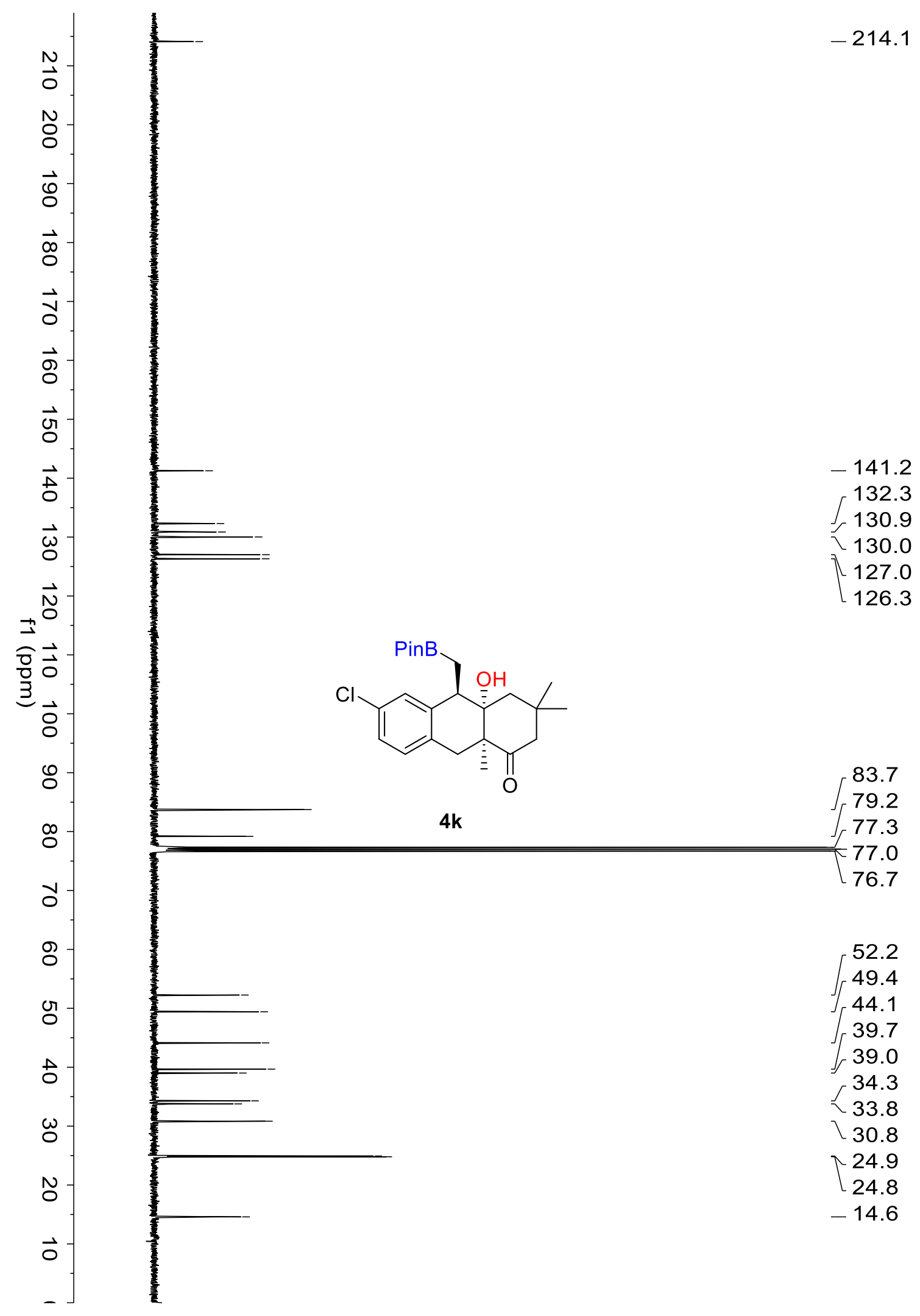




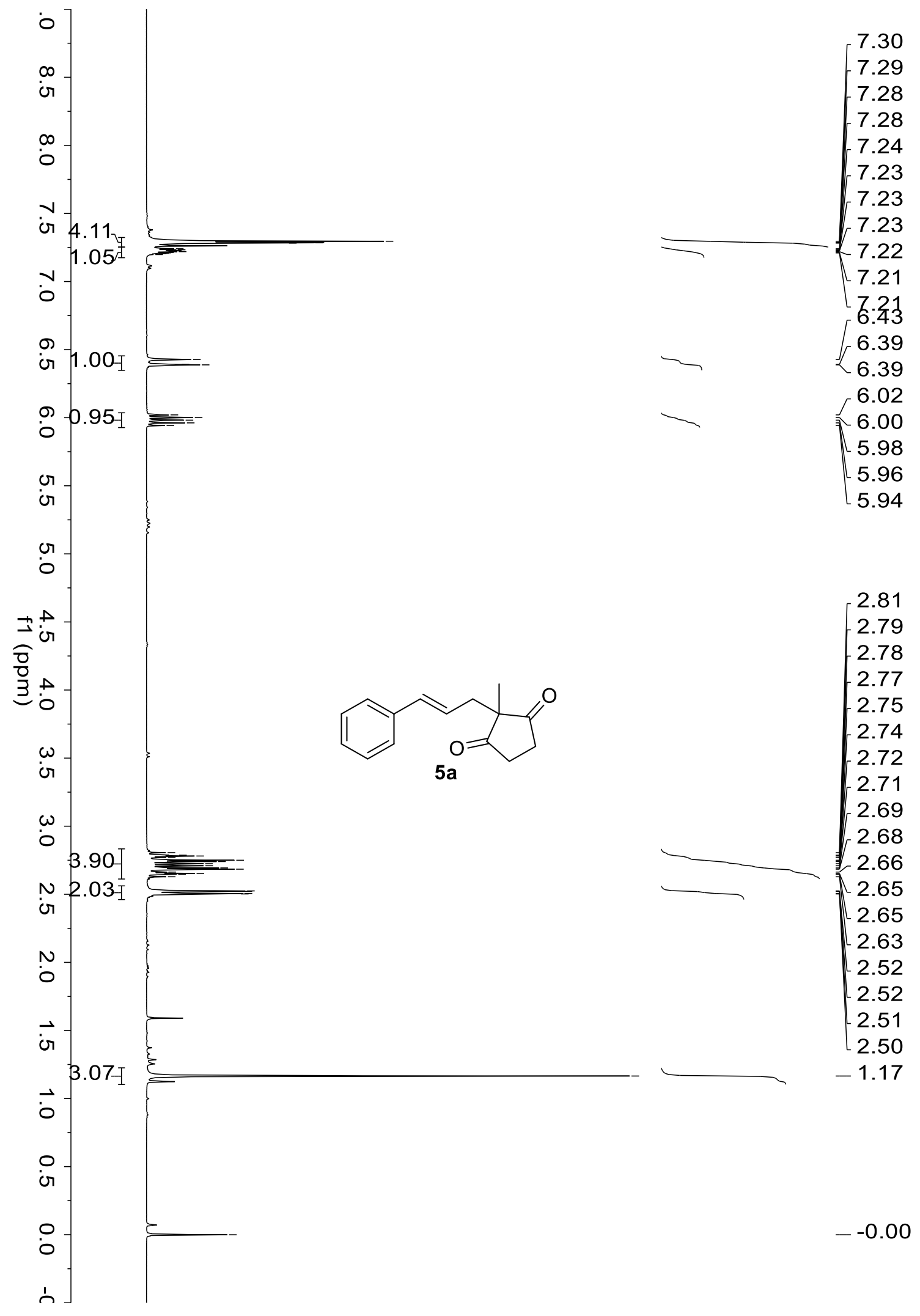




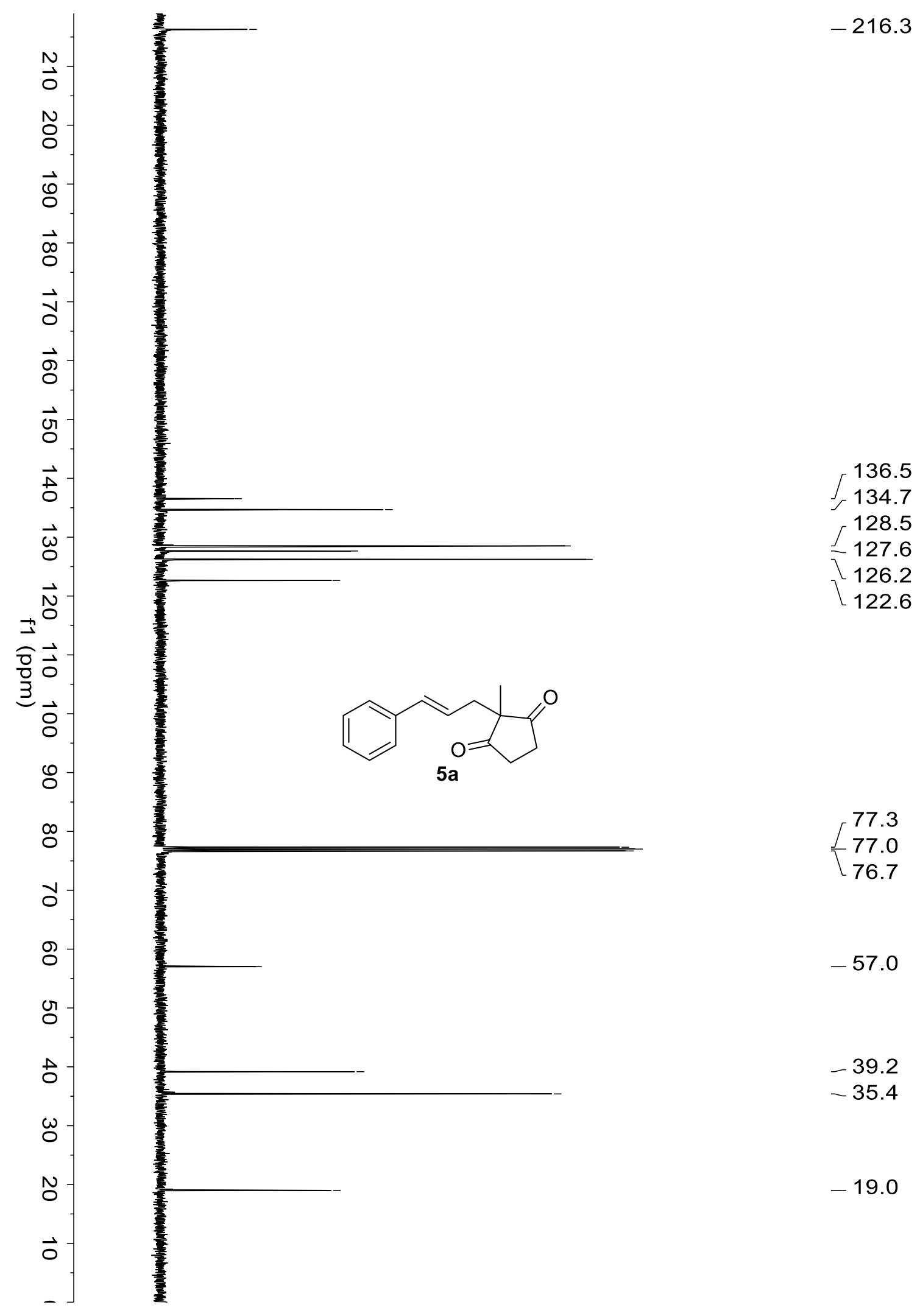




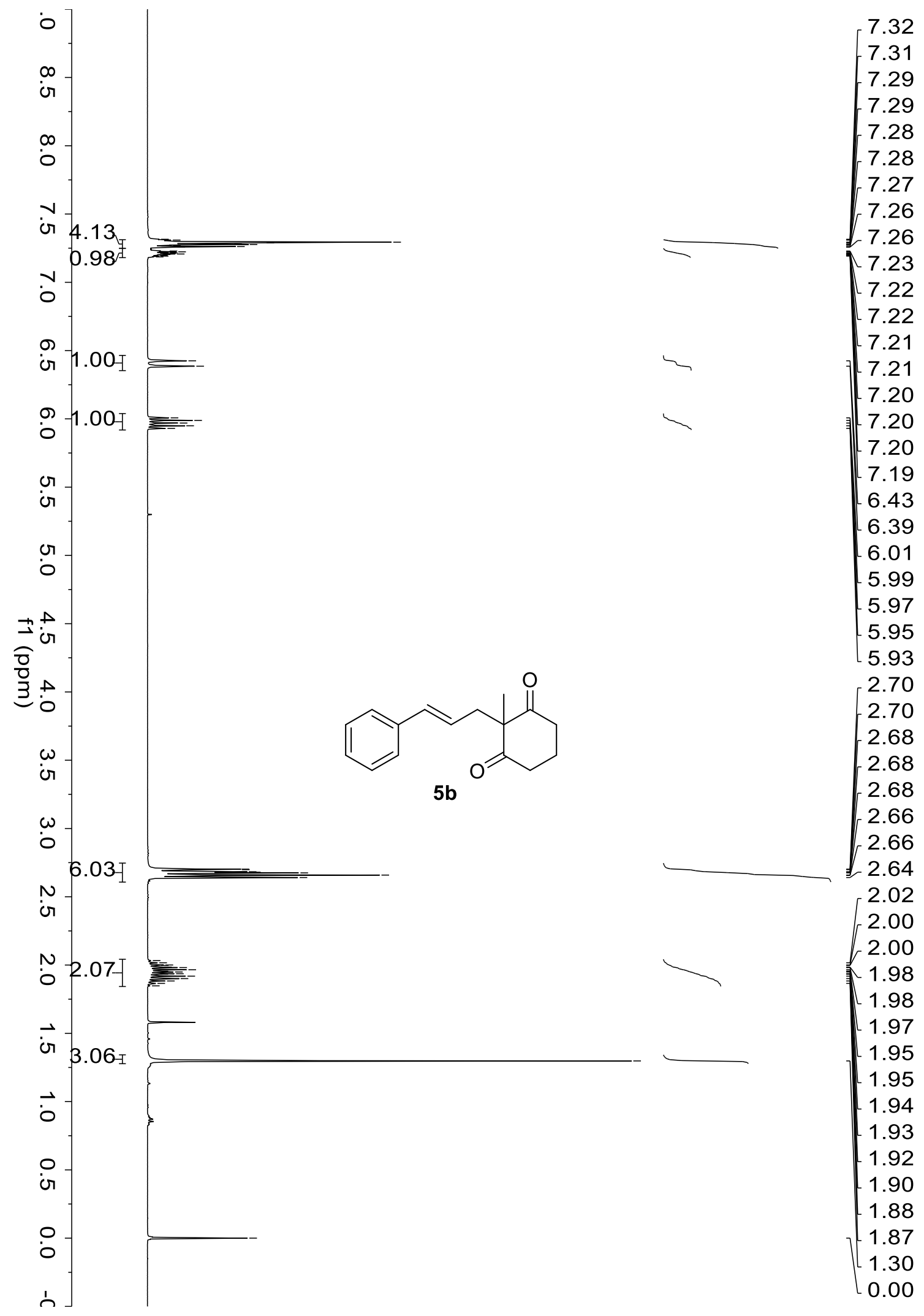




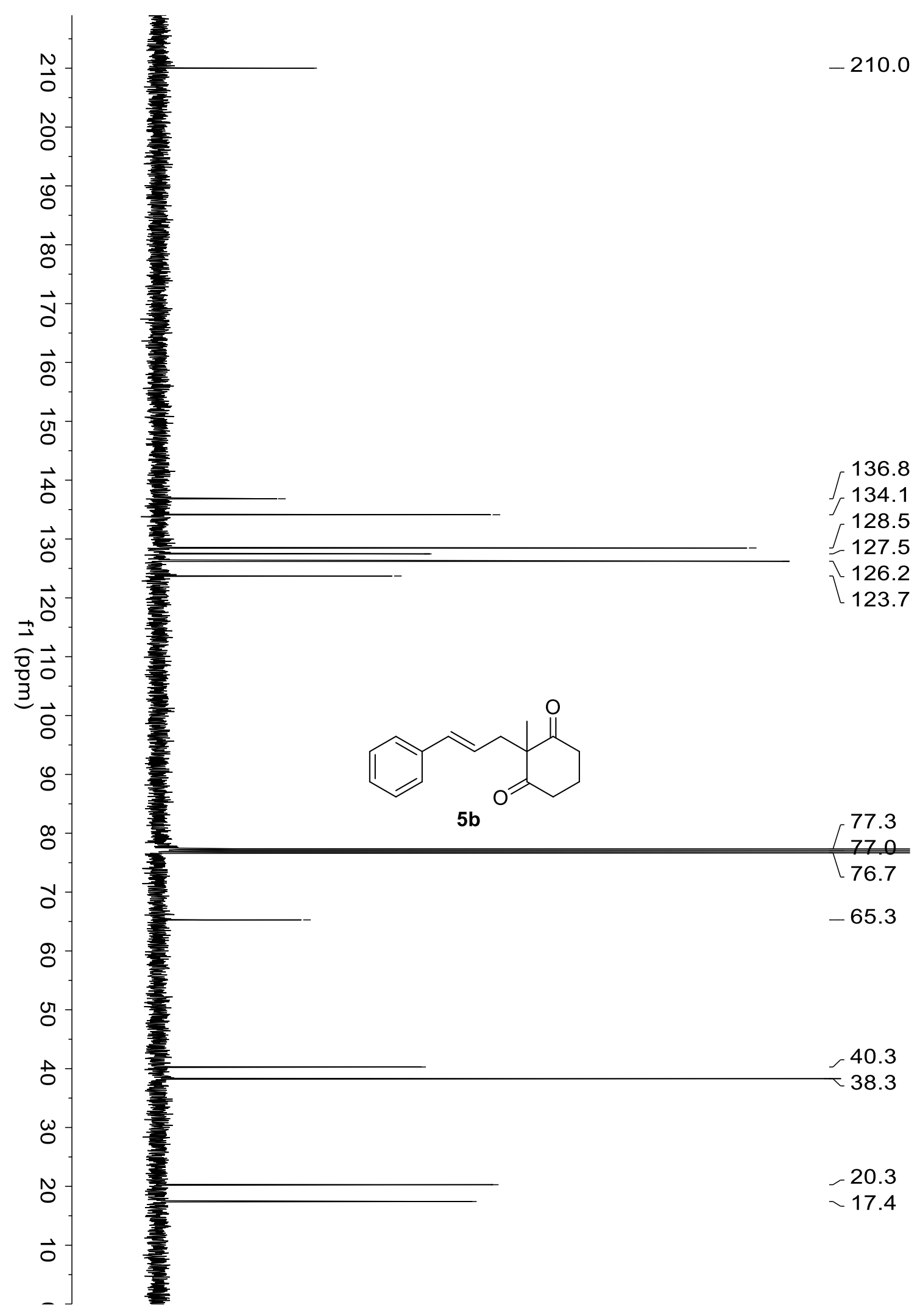



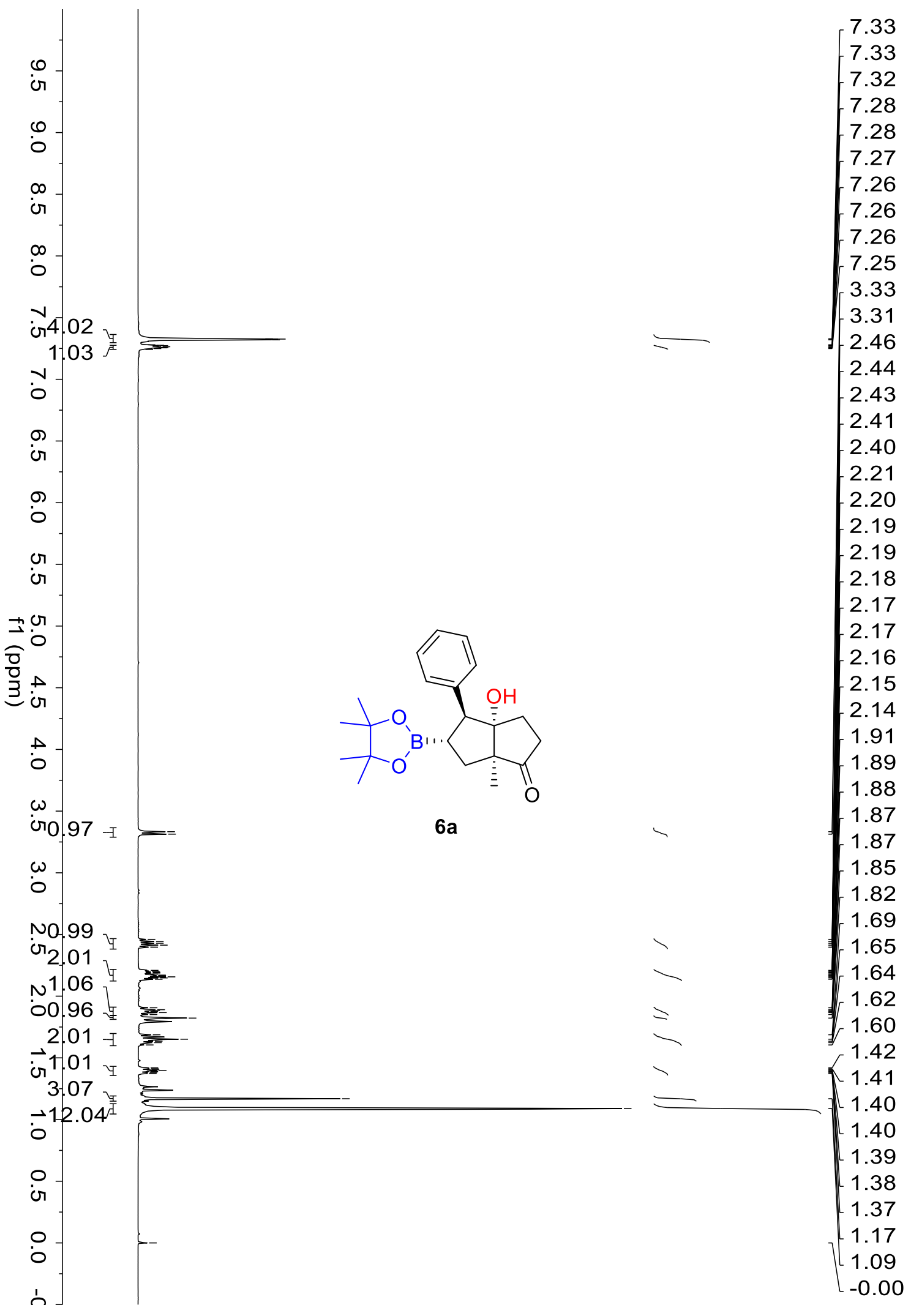


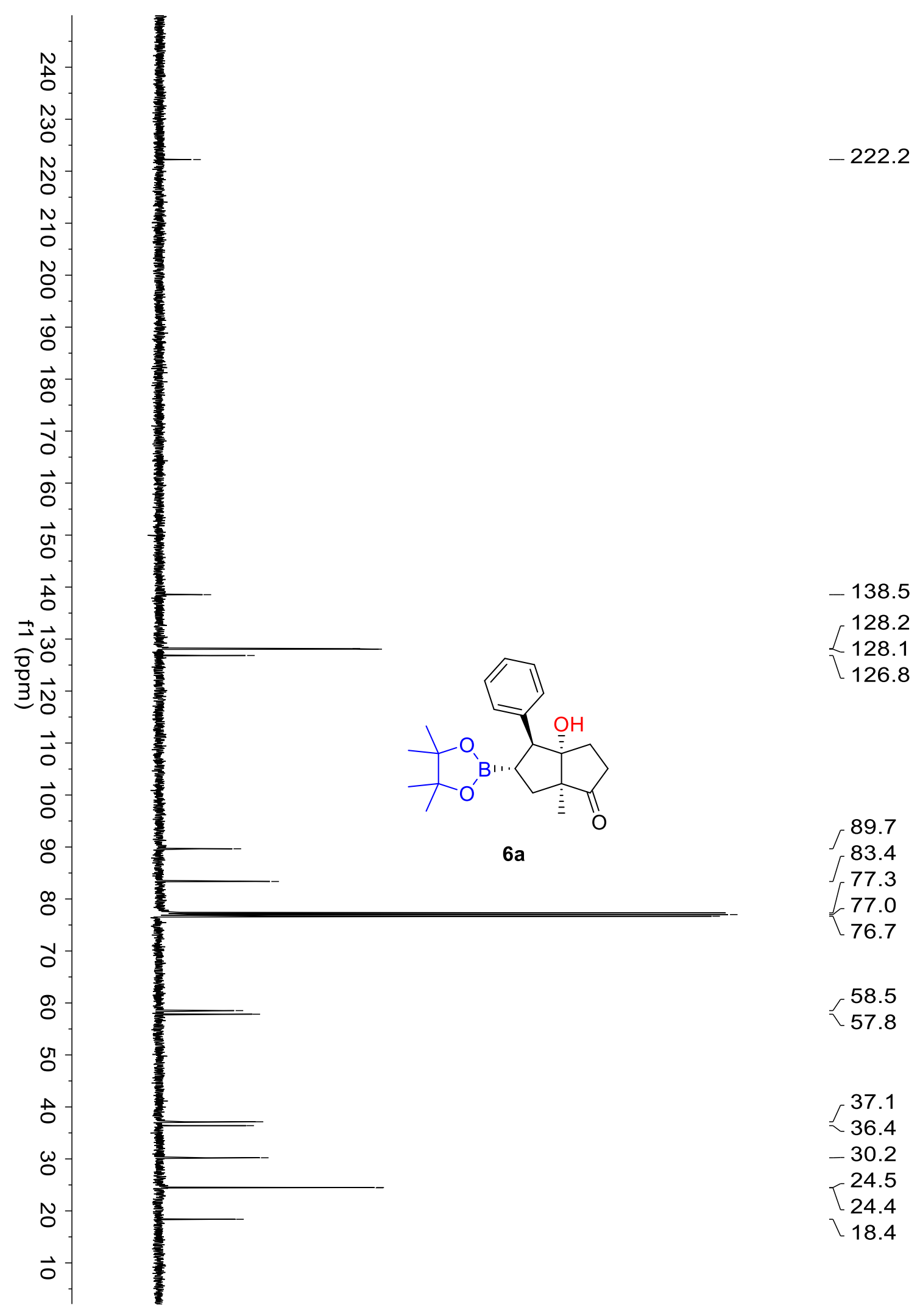




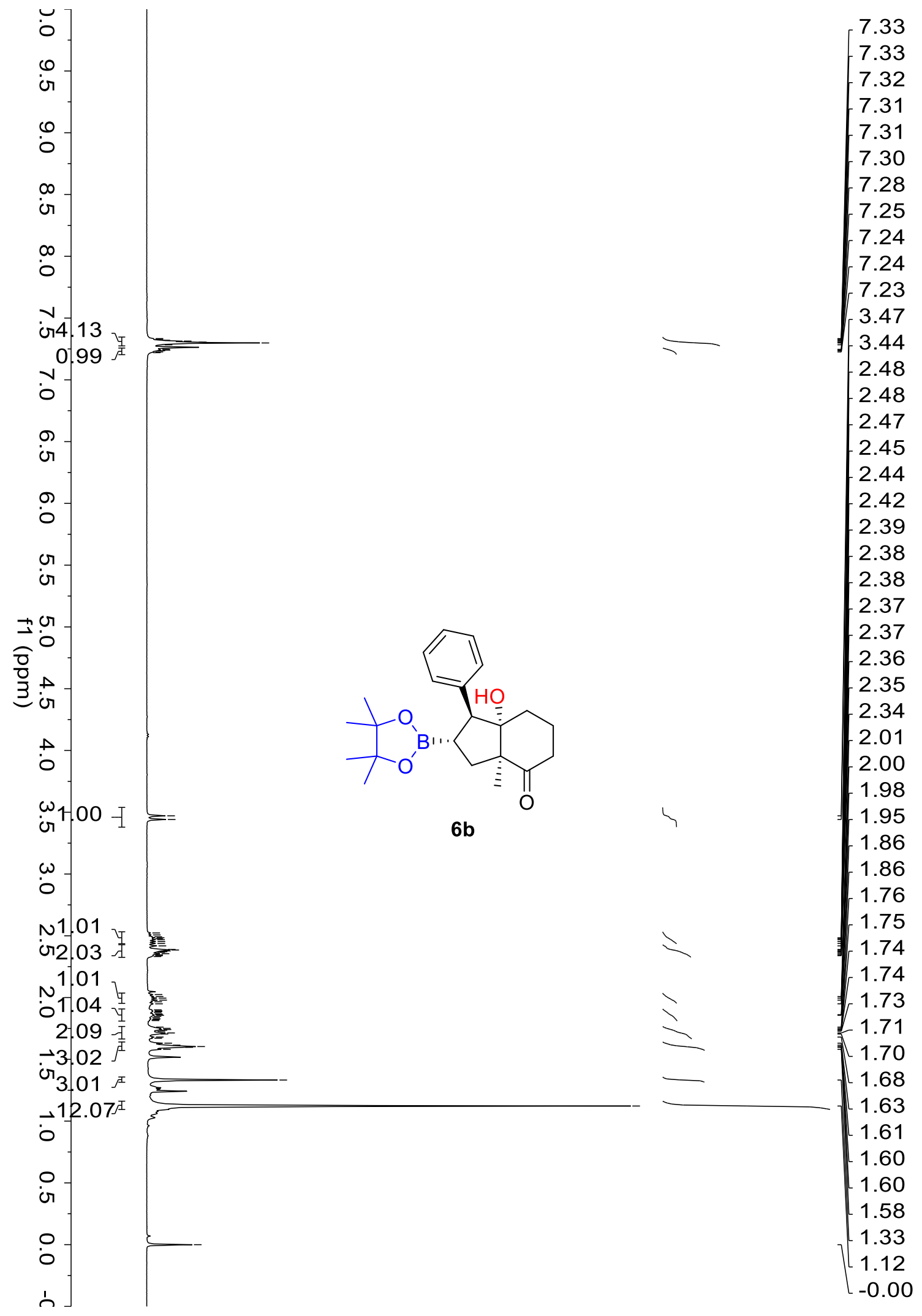




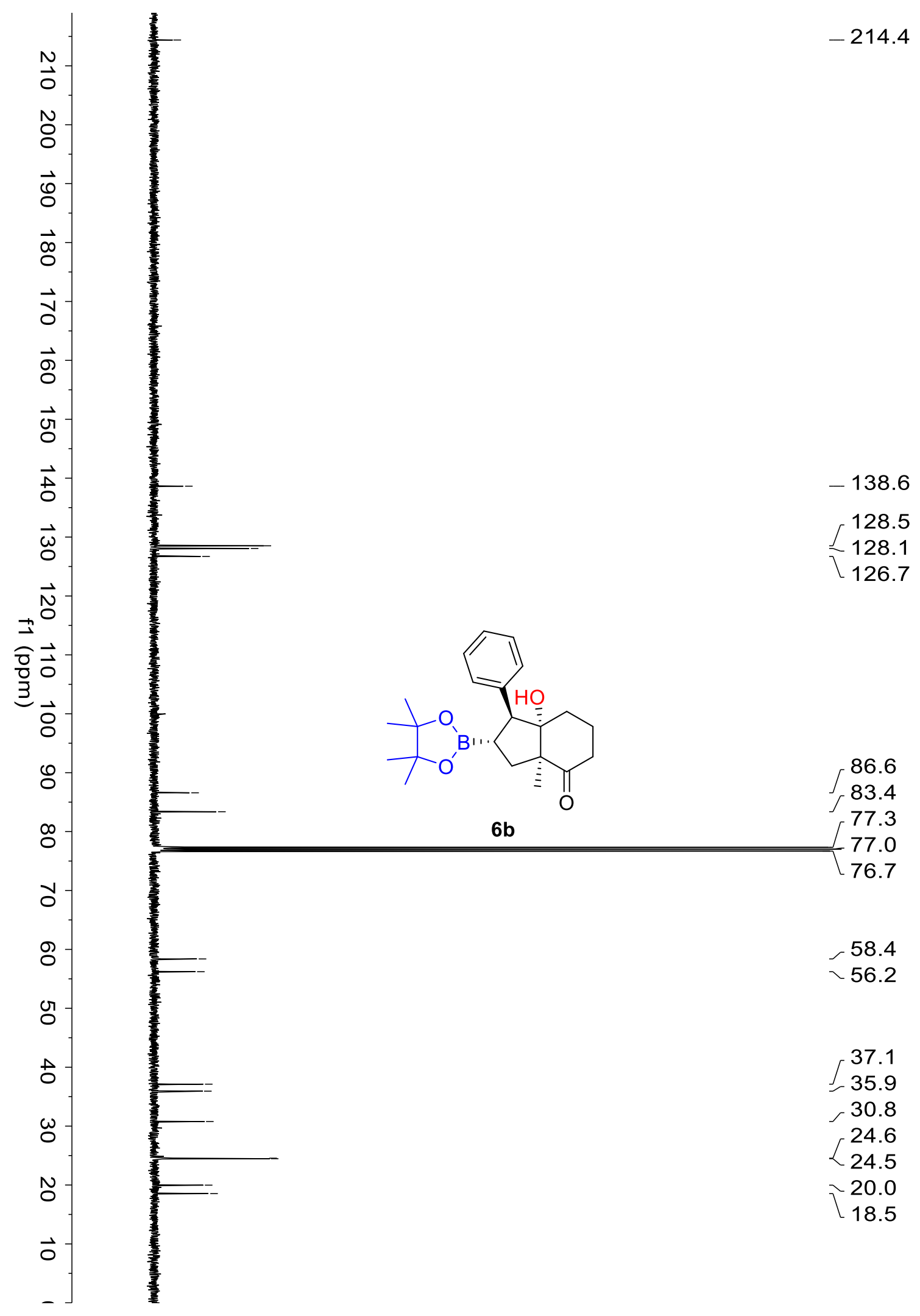




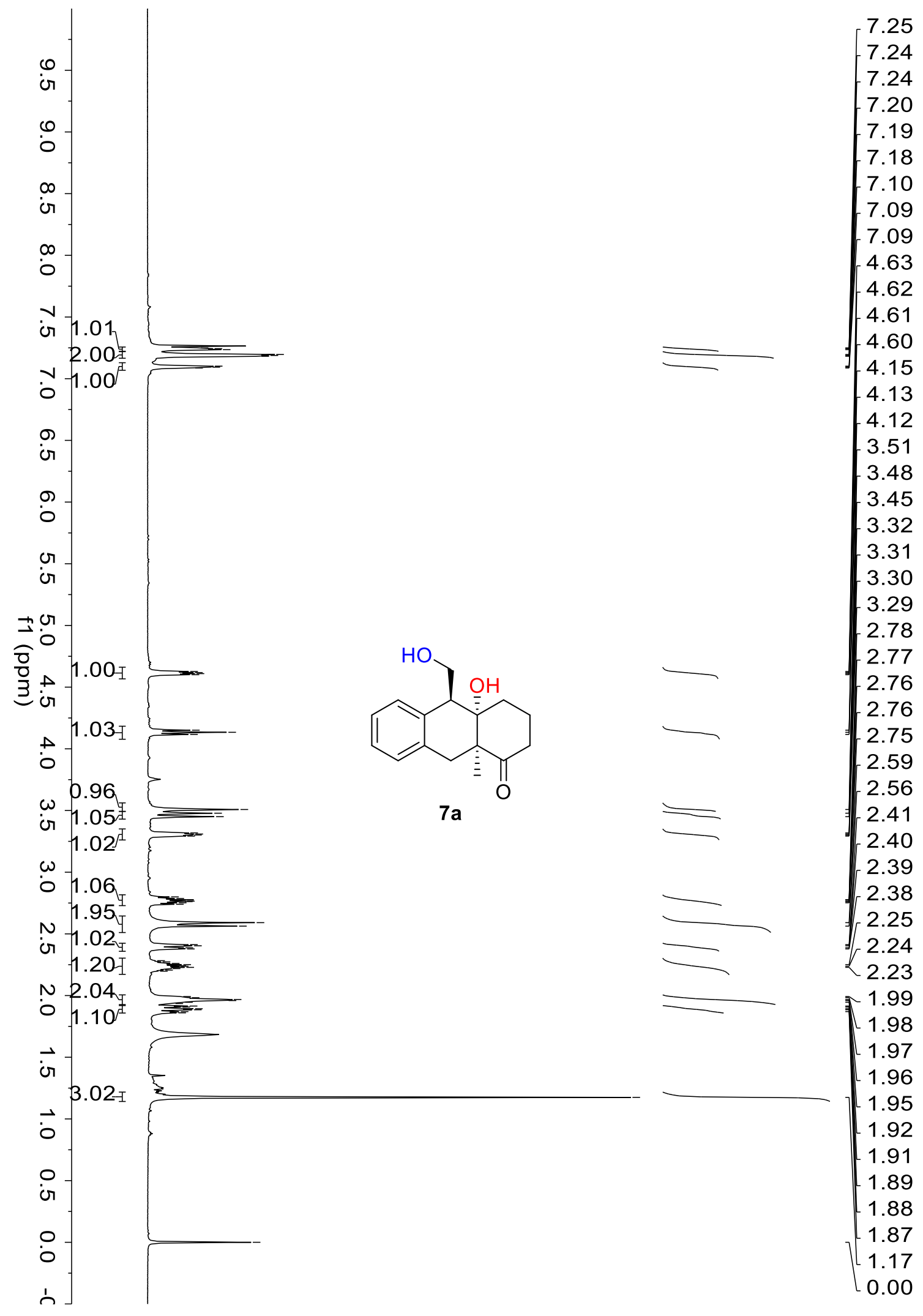




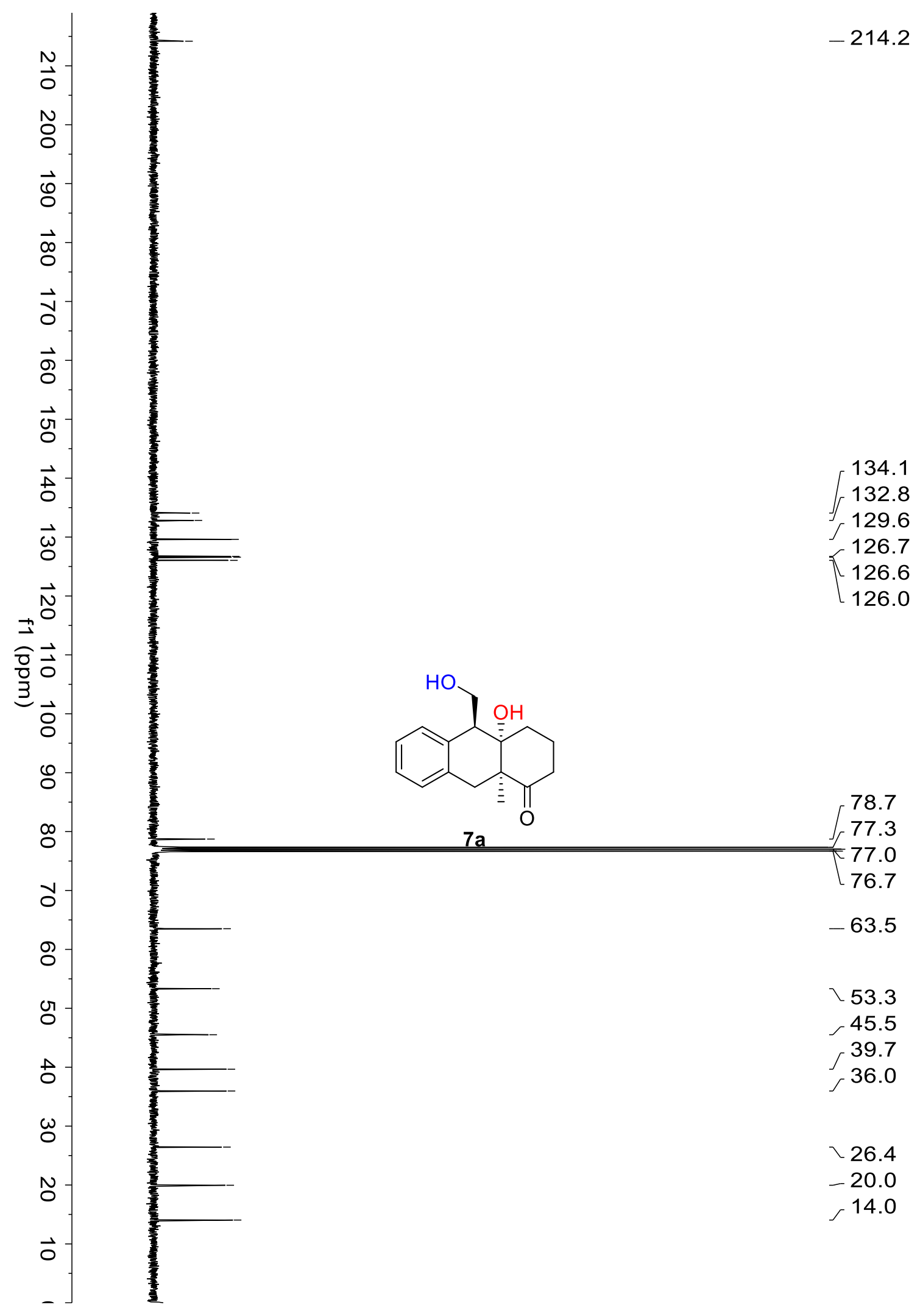




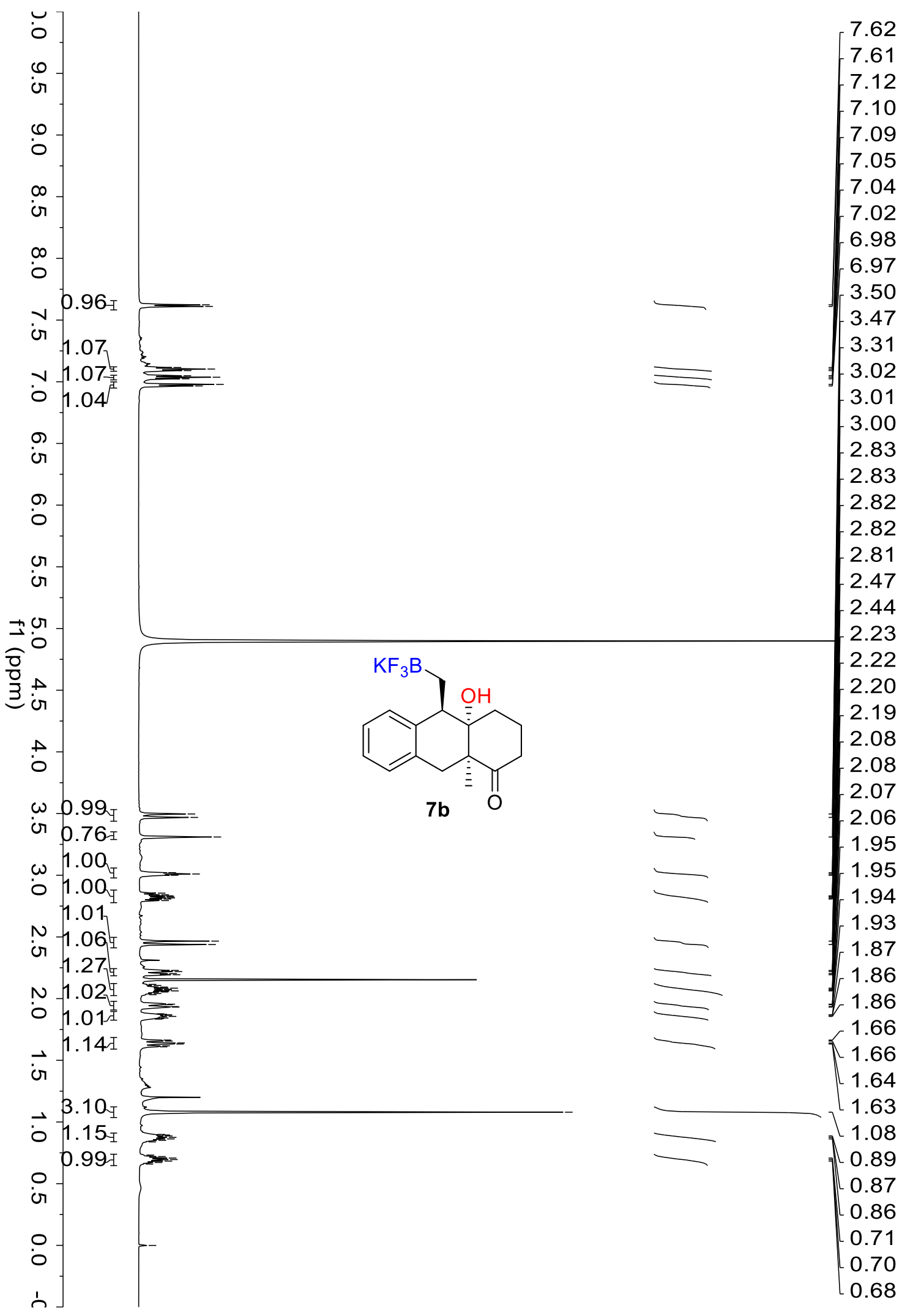




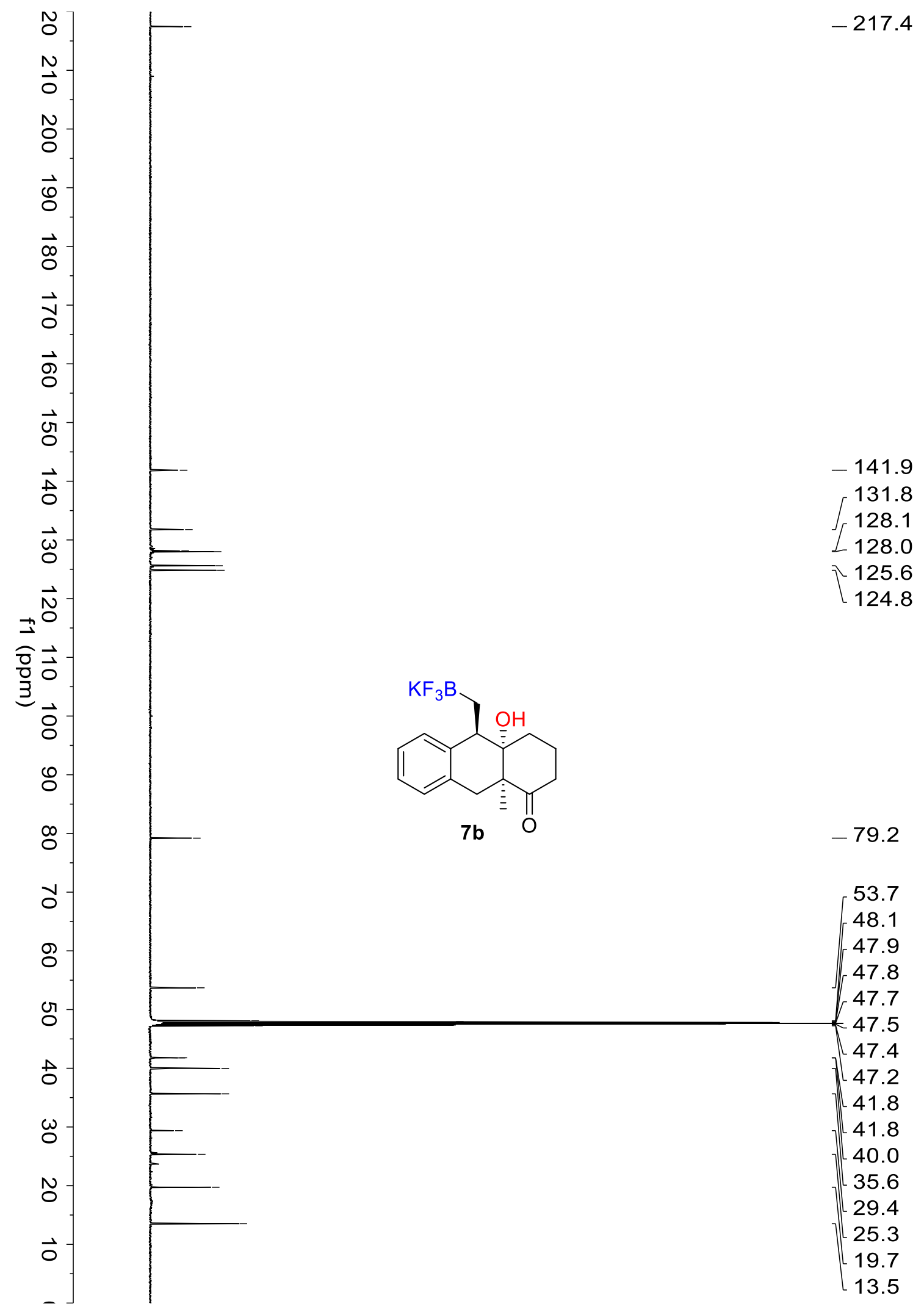



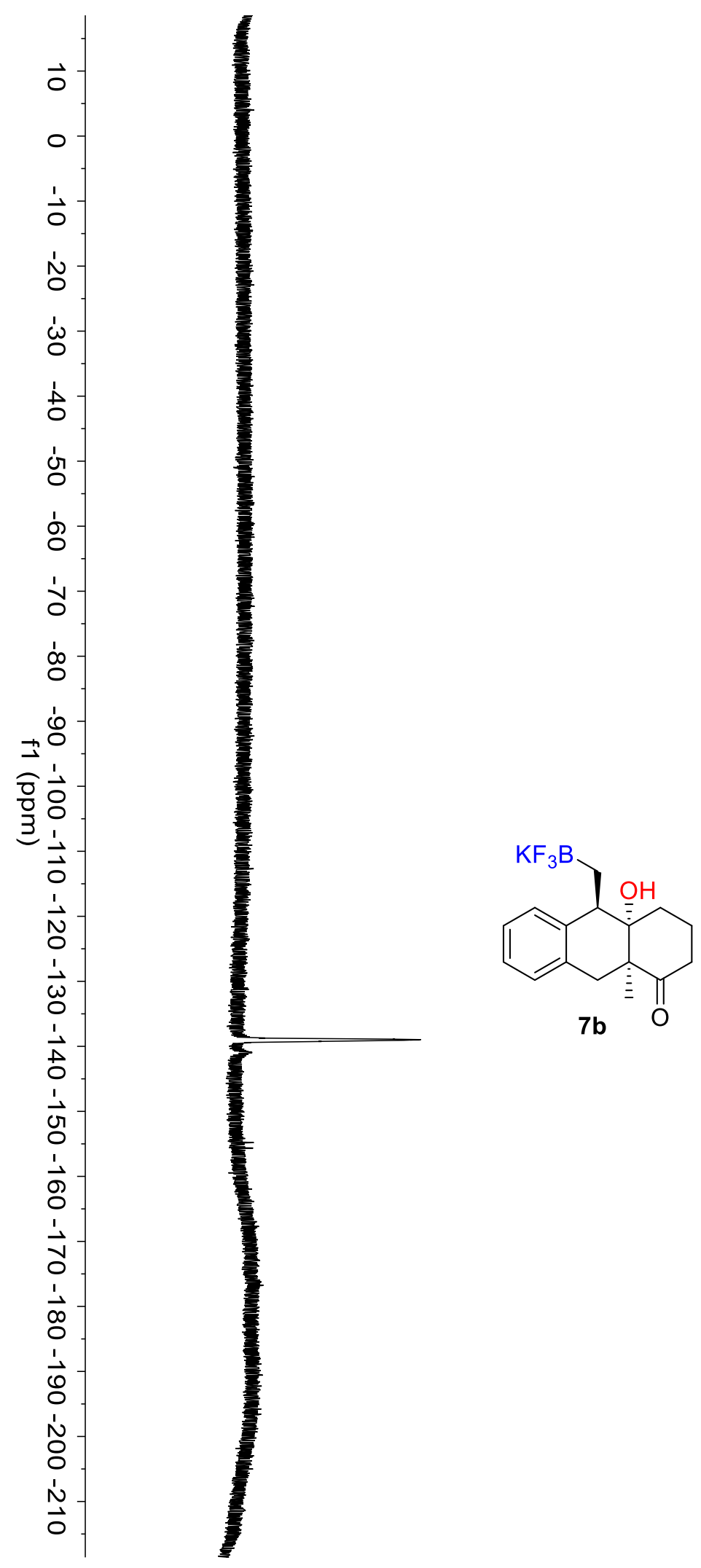

$--139.0$ 


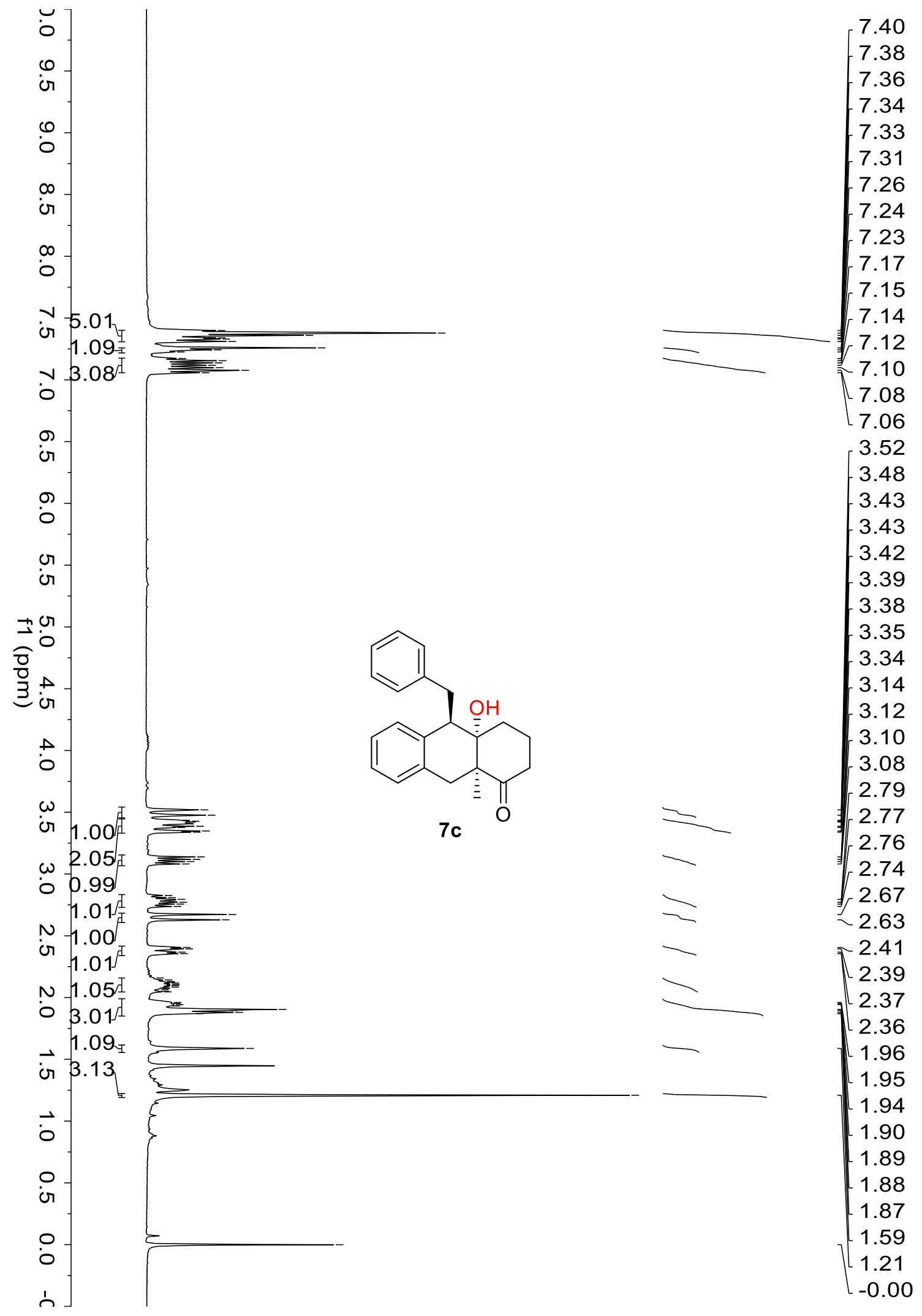




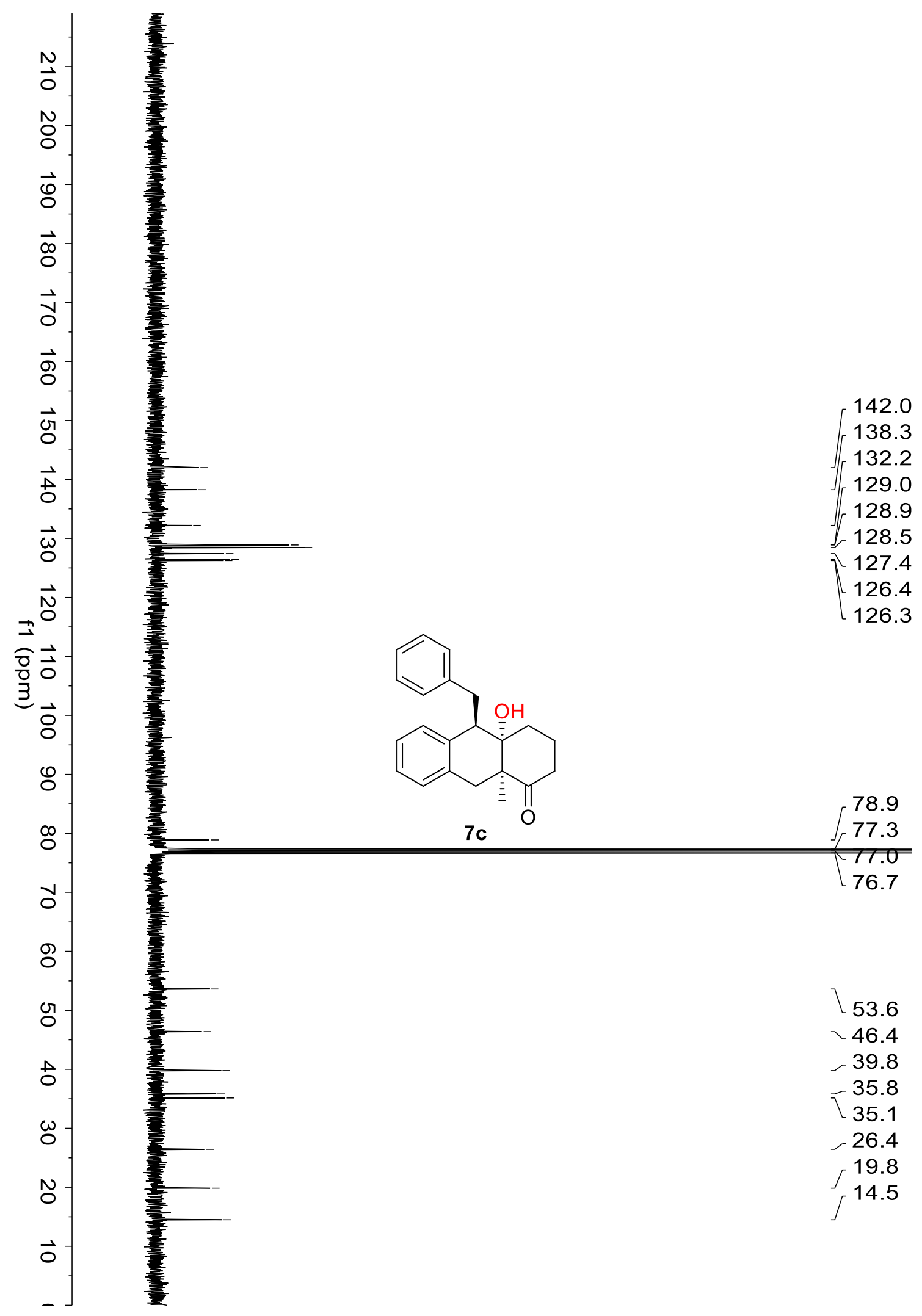



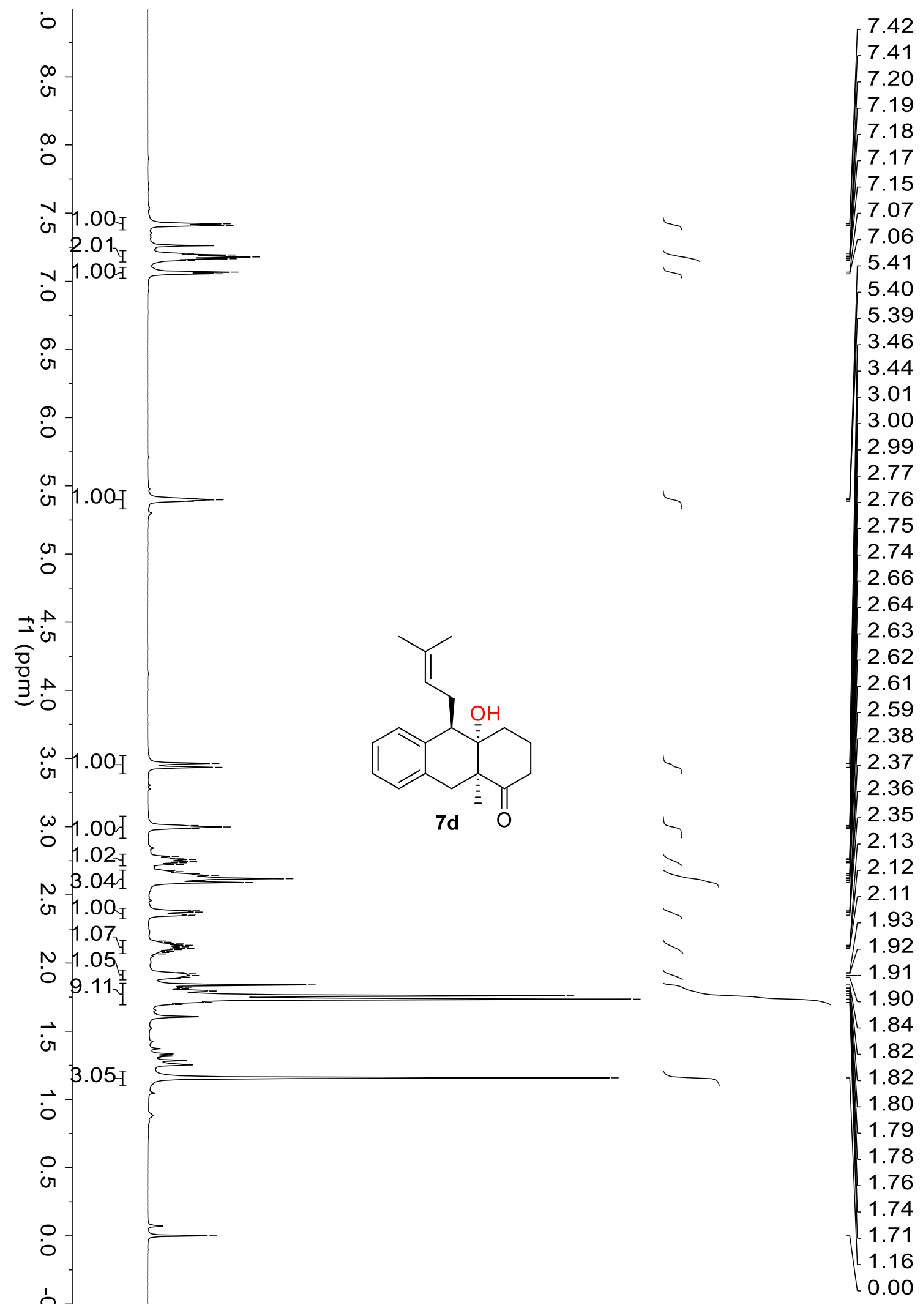


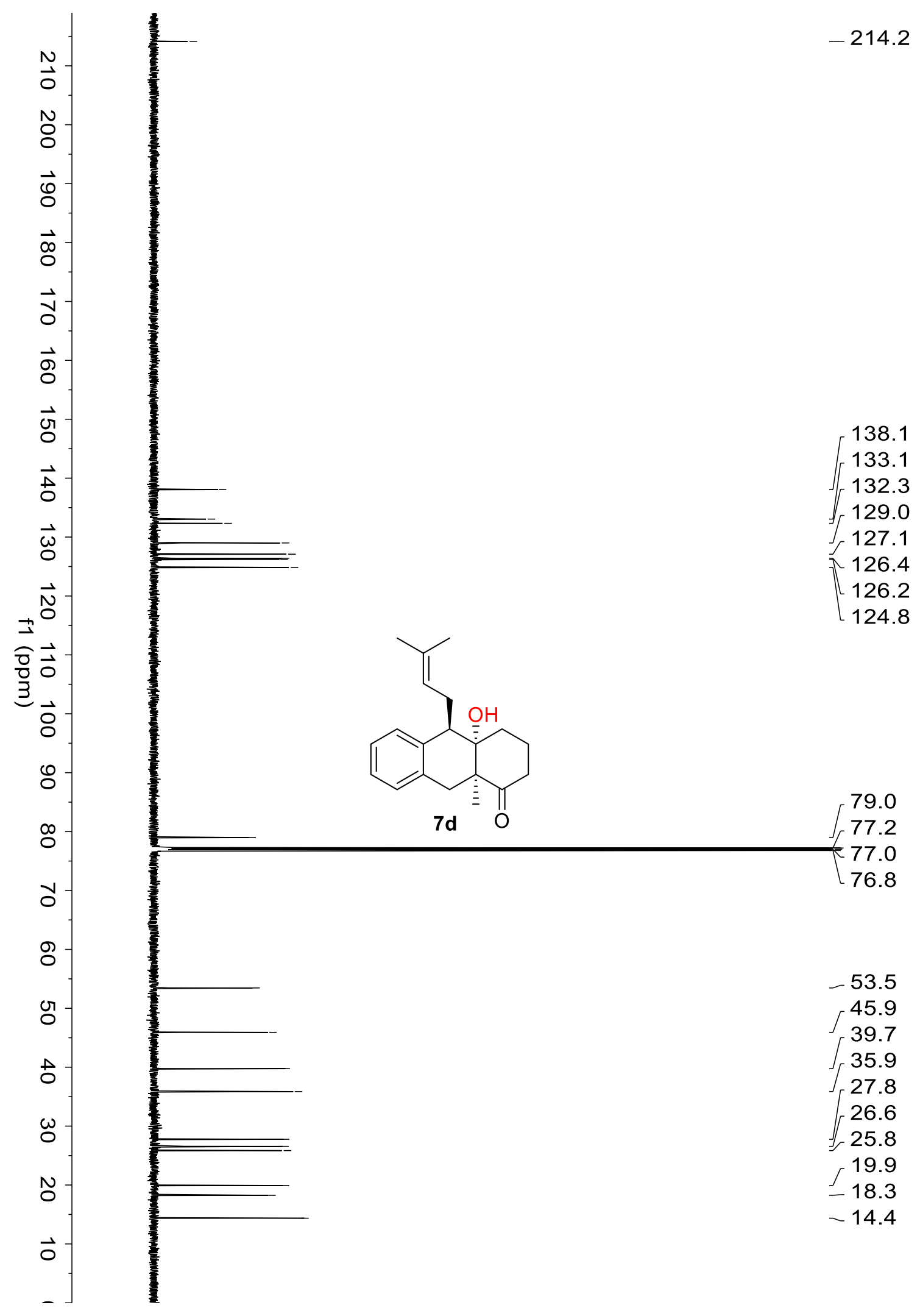

